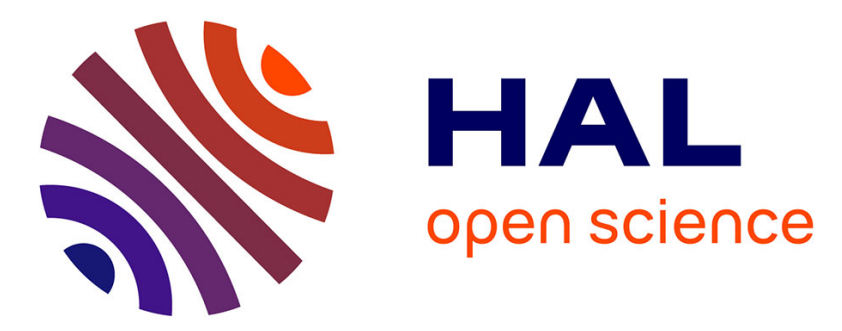

\title{
Sur les lois de comportement viscoplastique à variables internes - Exemples de deux alliages industriels: inoxydable austénitique 17-12 SPH et superalliage INCO718
}

P. Delobelle

\section{To cite this version:}

P. Delobelle. Sur les lois de comportement viscoplastique à variables internes - Exemples de deux alliages industriels: inoxydable austénitique 17-12 SPH et superalliage INCO718. Revue de Physique Appliquée, 1988, 23 (1), pp.1-61. 10.1051/rphysap:019880023010100 . jpa-00245747

HAL Id: jpa-00245747 https://hal.science/jpa-00245747

Submitted on 1 Jan 1988

HAL is a multi-disciplinary open access archive for the deposit and dissemination of scientific research documents, whether they are published or not. The documents may come from teaching and research institutions in France or abroad, or from public or private research centers.
L'archive ouverte pluridisciplinaire HAL, est destinée au dépôt et à la diffusion de documents scientifiques de niveau recherche, publiés ou non, émanant des établissements d'enseignement et de recherche français ou étrangers, des laboratoires publics ou privés. 


\title{
REVUE DE PHYSIQUE APPLIQUÉE
}

Revue Phys. Appl. 23 (1988) 1-61

JANVIER 1988, PAGE 1

Classification

Physics Abstracts

$46.00-46.30 \mathrm{~J}-62.00-62.20 \mathrm{~F}, \mathrm{H}-80.00-81.40 \mathrm{~L}$

\section{Sur les lois de comportement viscoplastique à variables internes}

\section{Exemples de deux alliages industriels : inoxydable austénitique 17- $12 \mathrm{SPH}$ et superalliage INCO718}

\author{
P. Delobelle
}

Laboratoire de Mécanique Appliquée, associé au CNRS, Faculté des Sciences et des Techniques, La Bouloie, route de Gray, 25030 Besançon Cedex, France

(Reçu le 16 février 1987, révisé le 25 juin 1987, accepté le $1^{\text {er }}$ septembre 1987)

Article de mise au point

Résumé. - Le but du présent article est d'effectuer un tour d'horizon sur les lois de comportement viscoplastique à variables internes développées en France au sein du GRECO "Grandes déformations et endommagement » et du GIS « Rupture à chaud ». On illustre, à l'aide de deux exemples d'alliages industriels (Inoxydable austénitique 17-12 SPH et Inconel 718), les comportements parfois complexes à modéliser. Cinq approches, d'origines différentes sont abordées, à savoir : deux unifiées, une partitionnée et deux approches multicouches (série et parallèle). On rappelle succinctement le cadre thermodynamique d'évolution de ces modèles qui conduit à la définition des différentes variables internes à partir des potentiels thermodynamiques (énergie libre $\psi$ ) et dissipatifs $(f$ et/ou $\Omega$ ). Chaque modèle est inséré dans ce contexte. Ainsi, pour l'ensemble de ces modèles, deux types de variables sont envisagés ; des variables cinématiques tensorielles d'ordre deux et des variables scalaires (isotropes). On montre comment l'analyse d'expériences bien réfléchies conduit à de nombreuses informations quant à la nature, la forme et l'identification des différentes fonctionnelles représentant le comportement (par exemple quant au choix de $\Omega$ et de l'éventuelle présence des termes de restauration intrinsèquement liés au temps). D'une façon non exhaustive, on présente quelques-unes des possibilités offertes par ces modèles. On conclut avec un paragraphe consacré aux développements futurs de ces approches, particulièrement dans le domaine des températures où les interactions dislocations-défauts ponctuels sont efficaces et pour le vaste champ des essais multiaxés hors phase.

Abstract. - The present paper is aimed at analysing the viscoplastic laws using internal variables worked out in France by the GRECO «Grandes déformations et endommagement " (large strains and damage), and the G.I.S. « rupture à chaud " (high temperature fracture). The behaviours, sometimes difficult to modelise, are described through the examples of two industrial alloys (stainless steels 17-12 SPH and Inco 718). Five different presentations will be considered, i.e. two unified models, a partitionning one and two multilayers (serie or parallel) ones. The thermodynamic basis of these models, together with the resulting definitions of the various internal variables derived from the thermodynamic potentials : free energy $\psi$ and dissipative potential $(f$ and/or $\Omega$ ), are reviewed. Every model is considered from this point of view. Thus, for all of them, two types of variables are introduced : kinemmatical (tensor of the second order) and isotropic (scalar) ones. The way to derive many an information about the nature, the form and the identification of the various functionals aimed at modelling the viscoplastic behaviour (for instance the choice of $\Omega$ or the possible occurrence of recovery terms intrinsically related to time) from the analysis of pertinent experiments is described. In a non exhaustive way some capabilities exhibited by these models are reported. A conclusing section is devoted to future developments of these approaches, specially in the cases of those temperature ranges where the point defectsdislocations interactions are efficient and in that of the large field of out of phase multiaxial tests. 
Notations et nomenclature.

* Notations générales

$Z \mathbf{Z}$
$\underline{\underline{\mathbf{z}}}$
$Z_{i j}^{\prime}$
$J_{2}(\underline{\mathbf{Z}})$
$\dot{Z}, Z^{\prime}(z)$
$\underline{\mathbf{Z}}^{\mathrm{p}}, \underline{\mathbf{z}}^{\mathrm{v}}$
$\underline{\mathbf{Z}}^{\mathrm{p}(n)}, \underline{\mathbf{z}}^{\mathrm{v}(n)}$
$\underline{\mathbf{z}}^{\mathrm{vm}}, \underline{\mathbf{z}}^{\mathrm{vM}}$
$\underline{\mathbf{z}}^{\mathrm{p}}(0), \underline{\mathbf{z}}^{\mathrm{v}}(0)$
exemple récapitulatif

$\underline{\mathbf{1}}, \underline{\underline{\mathbf{I}}}$

$\delta_{i j}$

$H(F)$

$\langle u\rangle$

$Z_{z z}, Z_{z \vartheta}$

* Grandeurs thermodynamiques :

$\psi$

$\stackrel{\omega}{\Omega, \Omega_{\mathrm{v}}}, \Omega_{\mathrm{r}}, \Omega_{\mathrm{v}}^{(n)}, \Omega_{\mathrm{r}}^{(n)}$

$f, f^{(n)}$

$\frac{\mathrm{m}}{\mathrm{g}}$ ou $\underline{\mathrm{n}}_{\mathrm{p}}, \underline{\mathrm{m}}^{(n)}$

\section{$F$}

$\rho$

$U$

$S$
: signification des symboles $\left({ }_{-},-,{ }^{\prime},,_{=}\right)$et des indices inférieurs et supérieurs.

: signifie que $Z$ est un scalaire.

: signifie que $\underline{\mathbf{Z}}$ est un tenseur d'ordre deux, en notation indicielle $Z_{i j}$.

: signifie que $\underline{\underline{Z}}$ est un tenseur d'ordre quatre, en notation indicielle $Z_{i j k l}$.

: composantes du déviateur du tenseur du second ordre $\underline{\mathbf{Z}}$, soit : $Z_{i j}^{\prime}=Z_{i j}-\delta_{i j} / 3 Z_{k k}$

: deuxième invariant du déviateur du tenseur $\mathbf{Z}$, soit : $J_{2}(\underline{\mathbf{Z}})=\left(\frac{3}{2} Z_{i j}^{\prime} Z_{i j}^{\prime}\right)^{1 / 2}$.

: dérivée par rapport au temps du scalaire $Z$ et dérivée de $Z$ par rapport à $(z)$.

: signifie que la variable $\underline{\mathbf{Z}}$ est intégrée dans un contexte plastique, indice $\mathrm{p}$, ou visqueux, indice $\mathrm{v}$, en notation indicielle $: Z_{i j}^{\mathrm{p}}, Z_{i j}^{\mathrm{v}}$.

: les variables $\underline{\underline{Z}}^{\mathrm{p}}$ et $\underline{\mathbf{Z}}^{\mathrm{v}}$ sont imputées à la couche $(n)$, dans le cas des modèles multicouches, en notation indicielle $Z_{i j}^{\mathrm{p}(n)}, Z_{i j}^{\mathrm{v}(n)}$.

Dans les modèles unifiés, l'indice $(n)$ signifie que l'on a plusieurs variables du même type.

: signifie dans le cas spécifique du modèle LMAB, que la variable $\underline{\underline{\mathbf{v}}}^{\mathbf{v}}$ décrit des microdéformations, indice $\mathrm{vm}$, ou des macrodéformations, indice vM. En notation indicielle $Z_{i j}^{\mathrm{vm}}, Z_{i j}^{\mathrm{vM}}$.

: valeur initiale du tenseur $\underline{Z}^{\mathrm{p}}$, ou $\underline{\mathbf{Z}}^{\mathrm{v}}$; en notation indicielle : $Z_{i j}^{\mathrm{p}}(0), Z_{i j}^{\mathrm{v}}(0)$.

$: J_{2}\left(\underline{\underline{Z}}^{\mathrm{vM}(2)}\right)=\left\{\frac{3}{2} Z_{i j}^{\mathrm{vM}(2)^{\prime}} Z_{i j}^{\mathrm{vM}(2)^{\prime}}\right\}^{1 / 2}$, signifie que l'on est en présence du deuxième invariant d'un tenseur $\underline{Z}$ du second ordre, qu'il s'agit d'un modèle visqueux, en particulier celui du modèle LMAB dans l'état macroviscoplastique, et que c'est la deuxième variable du type $\underline{\mathbf{Z}}$.

: tenseur unité du second ou du quatrième ordre, $I_{i j}, I_{i j k l}$.

: symbole de Kronecker.

: fonction échelon d'Heaviside soit : $H(F)=1$ si $F=0$ et $H(F)=0$ si $F<0$.

: signifie que $\langle u\rangle=u$ si $u \geqslant 0$ et $\langle u\rangle=0$ si $u<0$, soit $\langle u\rangle=u H(u)$.

: composante axiale et de cisaillement, en coordonnées cylindriques, du tenseur $\underline{\mathbf{z}}$.

: énergie libre.

: potentiel de dissipation.

: pseudo-potentiel de dissipation $\Omega$ décomposé en un potentiel visqueux $\Omega_{\mathrm{v}}$ et un potentiel de restauration $\Omega_{\mathrm{r}}$. Cas des multicouches : $\Omega_{\mathrm{v}}^{(n)}$ et $\Omega_{\mathrm{r}}^{(n)}$.

: surfaces seuils d'élasticité (globale ou affectée à une couche $(n))$.

: normales extérieures aux deux surfaces $f$ et $f^{(n)}$.

: surface seuil visqueuse (modèle EMP). Dans le modèle ONERA : $g \equiv f$.

: surface de charge-décharge exprimée dans l'espace des variables internes dans le cas du modèle LMAB.

: masse volumique.

: énergie interne.

: entropie du système. 
q

$D$

$A^{K}, \dot{a}^{k}$

$\mathcal{A}^{K^{\prime}}, \alpha^{k}$

$Y$
: flux de chaleur.

: dissipation intrinsèque.

: forces thermodynamiques $A^{K}$ associées aux flux $\dot{a}^{k}$.

: forces thermodynamiques $\mathcal{A}^{k}$ relatives à une famille de paramètres internes $\alpha^{k}$ de nature scalaire, ou tensorielle $\left(\mathcal{A}_{i j}^{k}, \alpha_{i j}^{k}\right)$.

: taux de restitution d'énergie d'endommagement.

* Variables et paramètres de déformation :

$\varepsilon^{\mathrm{T}}, \varepsilon^{\mathrm{el}}, \varepsilon^{\mathrm{p}}, \varepsilon^{\mathrm{v}}$

$\dot{\varepsilon}^{\mathrm{T}}, \dot{\varepsilon}^{\mathrm{p}}, \dot{\varepsilon}^{\mathrm{v}}$

$\dot{\varepsilon}^{\mathrm{st}}, \dot{\varepsilon}^{\mathrm{i}}$

$\underline{\boldsymbol{\varepsilon}}, \underline{\boldsymbol{\varepsilon}}^{\mathrm{p}}, \underline{\boldsymbol{\varepsilon}}^{\mathrm{v}}, \underline{\boldsymbol{\varepsilon}}^{\mathrm{p}(n)}, \underline{\boldsymbol{\varepsilon}}^{\mathrm{v}(n)}$

\section{$\bar{\varepsilon}, \overline{\dot{\varepsilon}}$}

$\dot{p}, \dot{v}$

$p, v$

$\lambda, \lambda^{(n)}$

$I_{2}\left(\underline{\boldsymbol{\varepsilon}}^{\mathrm{v}}\right)$

G

$\xi$

$q$

in*

$\eta, g, \mu$

\section{* Les contraintes:}

$\sigma, \sigma_{z z}$

$\dot{\sigma}$

$\sigma_{\mathrm{i}}$

$\sigma_{\mathrm{v}}$

$\underline{\boldsymbol{\sigma}}$

$\underline{\mathbf{\sigma}}^{(n)}$

$J_{2}(\underline{\boldsymbol{\sigma}})$
: déformations, totale, élastique, plastique et visqueuse en $1 \mathrm{D}$. : vitesses de déformation, totale, plastique et visqueuse en $1 D$.

: vitesses de déformation stationnaire et instantanée en $1 \mathrm{D}$.

: tenseur du second ordre des déformations. Respectivement, lorsque l'on ne connaît pas a priori la nature de la déformation, plastique, visqueuse, plastique dans la couche $(n)$ et visqueuse dans la couche $(n)$. En notation indicielle : $\varepsilon_{i j}, \varepsilon_{i j}^{\mathrm{p}}, \varepsilon_{i j}^{\mathrm{v}}, \varepsilon_{i j}^{\mathrm{p}(n)}$ et $\varepsilon_{i j}^{\mathrm{v}(n)}$.

: déformation et vitesse de déformation équivalente au sens de Misès, voir $I_{2}\left(\underline{\varepsilon}^{v}\right)$, lorsque l'on ne connaît pas a priori la nature de la déformation (absence d'indice).

: vitesses de déformation équivalente, plastique et visqueuse $\dot{p}=\left(\frac{2}{3} \dot{\varepsilon}_{i j}^{\mathrm{p}_{1 j}} \dot{\varepsilon}_{l j}^{\mathrm{p}}\right)^{1 / 2}$ et $\dot{v}=\left\{\frac{2}{3} \dot{\varepsilon}_{i j}^{\mathrm{v}} \dot{\varepsilon}_{i j}^{\mathrm{v}}\right\}^{1 / 2}$.

: déformation équivalente cumulée plastique et visqueuse $p=$ $\int_{0}^{t}\left\{\frac{2}{3} \dot{\varepsilon}_{i j}^{\mathrm{p}} \dot{\varepsilon}_{i j}^{\mathrm{p}}\right\}^{1 / 2} \mathrm{~d} t$ et $v=\int_{0}^{t}\left\{\frac{2}{3} \dot{\varepsilon}_{i j}^{\mathrm{v}} \dot{\varepsilon}_{i j}^{\mathrm{v}}\right\}^{1 / 2} \mathrm{~d} t$.

: multiplicateur plastique défini par une équation de consistance.

: deuxième invariant du tenseur des déformations visqueuses : $I_{2}\left(\underline{\varepsilon}^{\mathrm{v}}\right)=\left(\frac{2}{3} \varepsilon_{i j}^{\mathrm{v}} \varepsilon_{i j}^{\mathrm{v}}\right)^{1 / 2}$, identique à $\bar{\varepsilon}^{\mathrm{v}}$.

: surface de non-écrouissage exprimée dans l'espace des déformations.

: variable de déformation cinématique associée à $G ;\left(\xi_{i j}\right)$.

: variable isotrope associée au rayon de la surface $G$.

: normale à la surface $G$.

: paramètres affectant les variables $\underline{\xi}$ et $q$ de la surface $G$.

: contrainte uniaxiale : quelconque et suivant l'axe $Z Z$.

: vitesse en contrainte.

: contrainte interne en $1 \mathrm{D}$, au sens large.

: composante visqueuse.

: tenseur des contraintes; en notation indicielle $\sigma_{i j}$.

: tenseur des contraintes locales dans le sous-matériau $(n)$ : $\sigma_{i j}^{(n)}$.

: conformément aux notations générales, il s'agit du deuxième invariant du déviateur des contraintes; $J_{2}(\underline{\mathbf{\sigma}})=$ $\left(\frac{3}{2} \sigma_{i j}^{\prime} \sigma_{i j}^{\prime}\right)^{1 / 2}$

* Variables et paramètres cinématiques :

$\underline{\mathbf{X}}^{\mathrm{p}}, \underline{\mathbf{X}}^{\mathrm{v}}, \underline{\mathbf{X}}^{\mathrm{p}(n)}, \underline{\mathbf{X}}^{\mathrm{v}(n)}$

: tenseur du second ordre représentant la variable d'écrouissage cinématique plastique ou visqueuse; en notation indicielle $X_{i j}^{\mathrm{p}}, X_{i j}^{\mathrm{v}}, X_{i j}^{\mathrm{p}(n)}, X_{i j}^{\mathrm{v}(n)}$.

$\underline{\boldsymbol{\alpha}}^{\mathrm{p}}, \underline{\boldsymbol{\alpha}}^{\mathrm{v}}, \underline{\boldsymbol{\alpha}}^{\mathrm{vm}}, \underline{\boldsymbol{\alpha}}^{\mathrm{vM}(n)}$

: paramètres internes extensifs associés aux variables cinématiques intensives $\underline{X}^{(+)}$: en notation indicielle $\alpha_{i j}^{\mathrm{p}}, \alpha_{i j}^{\mathrm{v}}, \alpha_{i j}^{\mathrm{vm}}, \alpha_{i j}^{\mathrm{vM}(n)}$.

$J_{2}\left(\underline{\mathbf{X}}^{\mathrm{v}}\right), J_{2}\left(\underline{\mathbf{X}}^{\mathrm{p}}\right), J_{2}\left(\underline{\boldsymbol{\alpha}}^{\mathrm{v}}\right), J_{2}\left(\underline{\mathbf{\alpha}}^{\mathrm{p}}\right)$ 
$J_{2}\left(\underline{\mathbf{\sigma}}-\underline{\mathbf{X}}^{\mathrm{p}}\right), J_{2}\left(\underline{\mathbf{\sigma}}-\underline{\mathbf{X}}^{\mathrm{v}}\right)$

$a, a^{\mathrm{v}}, a^{\mathrm{p}}, a^{\mathrm{v}(n)}, a^{\mathrm{p}(n)}, a^{*}, b^{*}, a^{(n)}$

$a_{\mathrm{n} 1}^{\mathrm{p}}, A_{(\mathrm{v})}^{\mathrm{v}}, A_{(\mathrm{p})}^{\mathrm{p}}, A, B$

$C^{\mathrm{v}}, C^{\mathrm{p}}, C_{\mathrm{s}}, C^{(n)}$

$K^{\mathrm{v}}, K^{\mathrm{p}}, K^{\mathrm{vp}}, p_{2}$

$r,(l=r), M$

$\beta, \beta^{\prime}, R_{0}, R_{2}$

$\underline{\mathbf{X}}_{0}^{\mathrm{vM}(2)}$

$\underline{\mathbf{X}}_{0}^{\mathrm{p}(n)}, \underline{\mathbf{X}}_{0}^{\mathrm{p}(l)}$

* Variables et paramètres isotropes :

$R^{\mathrm{p}}, R^{\mathrm{v}}, R^{\mathrm{p}(n)}, R^{\mathrm{v}(n)}$

$R_{0}^{\mathrm{p}}, R_{0}^{\mathrm{v}}, R_{0}^{\mathrm{p}(n)}, R_{0}^{\mathrm{v}(n)}$

$r^{\mathrm{p}}, r^{\mathrm{v}}$

$R^{*}, \mathcal{R}^{v}, \mathbb{R}^{*}$

$R_{i j}^{\mathrm{p}}$

$W\left(r^{\mathrm{p}}\right), W\left(r^{v}\right)$

$b, b^{\mathrm{v}}, b^{\mathrm{p}}, b^{(n)}$

$Q_{1}, Q \mathrm{P}, Q_{1}^{\mathrm{v}}, Q^{(n)}, Q_{1}^{\text {sat }}, Q_{0}^{\text {sat }} Q_{\infty}^{\text {sat }}, Q_{1}^{\text {sat }}\left(A^{*}\right)$

$l$

$\gamma, \gamma^{\mathrm{v}}, \gamma^{\mathrm{p}}, R_{1}$

$Q_{2}$

* Grandeurs physiques :

$T$

$T_{\mathrm{F}}$

$T_{\mathrm{f}}$

$T_{(\mathrm{i})}$

$t$

$t_{\mathrm{m}}$

$\Delta H$

$k$

$D_{\mathrm{Fe}}$

$E, G, \nu$

$b$

$\alpha *(T)$

$\tau_{\mathrm{SN}(\mathrm{i})}$
: voir notations générales ; soit en explicitant en notation indicielle : $J_{2}\left(\underline{\mathbf{\sigma}}-\underline{\mathbf{X}}^{\mathrm{p}}\right)=\left\{\frac{3}{2}\left(\sigma_{i j}^{\prime}-X_{i j}^{\mathrm{p}^{\prime}}\right)\left(\sigma_{i j}^{\prime}-X_{i j}^{\mathrm{p}^{\prime}}\right)\right\}^{1 / 2}$.

: constantes en facteur des termes d'écrouissage linéaire (ex. : $\dot{X}_{i j}=a^{(+)} \dot{\varepsilon}_{i j}$ ) suivant les différents modèles.

: paramètres entrant dans la partie évanescente de l'écrouissage cinématique non linéaire (ex. : $\left.-C^{\mathrm{p}} X_{i j}^{\mathrm{p}} \dot{p}\right)$, suivant les modèles.

: constantes en facteur des termes d'écrouissage cinématique non linéaire dans les modèles de l'EMP et LMAB (ex. : $K^{\mathrm{p}}\left(a^{\mathrm{p}} \varepsilon_{i j}^{\mathrm{p}}-C^{\mathrm{p}} \dot{p}\right)$.

: exposants intervenant dans les termes de restauration.

: constantes multiplicatives intervenant dans les termes de restauration.

: valeur maximale non restaurable de la variable cinématique dans le modèle LMAB.

: valeur maximale non restaurable des variables cinématiques dans les modèles CEA et LMX.

: variables isotropes dans les différentes approches.

: valeurs constantes, égales aux valeurs initiales des variables isotropes.

: paramètres internes associés aux variables isotropes $R^{\mathrm{p}}$ et $R^{\mathrm{v}}$.

: variables internes isotropes associées aux effets de microstructure.

: fonction de distorsion relative à la description des surfaces de charge.

: fonctions scalaires intervenant dans l'écriture de l'énergie libre et conduisant à la cinétique de la variable $R^{\mathrm{v}, \mathrm{p}}$.

: constante affectant la rapidité de la cinétique du premier ordre de la variable isotrope dans les diverses approches.

valeurs asymptotiques de la variable isotrope, sans ou avec définition de zone de non-écrouissage et dans le cas des chargements hors phase.

: exposant affecté au terme de restauration de la variable isotrope.

: constantes multiplicatives intervenant dans les termes de restauration.

: valeur maximale non restaurable de la variable isotrope.

: température actuelle.

: température de fusion.

: température fictive (variable interne dans les effets microstructuraux).

: température caractéristique de l'interaction à courte distance (i).

: temps.

: temps de maintien à une valeur de consigne.

: énergie d'activation.

: constante de Boltzman.

: coefficient de diffusion du fer au sein de la solution solide.

: module d'Young, de Coulomb et coefficient de Poisson.

: vecteur de Burgers.

: coefficient de dilatation linéaire.

: temps de relaxation de Snoek du soluté (i). 
$W(\mathrm{i})$

$W_{\mathrm{cxt}}, W_{\mathrm{c}}, W_{\mathrm{a}}, W_{\mathrm{b}}, W_{\mathrm{c}}$

$\mathcal{F}$

$v, \vartheta$

* Autres grandeurs :

$n^{*}$

$K_{0}, \alpha_{k}$

$w^{(n)}$

$\stackrel{\underline{\underline{A}}}{ }^{+}$

$\Delta \sigma_{\mathrm{r}}$

$\mathbb{D}, \mathbb{D}$
: énergie d'activation du temps de relaxation de l'atome soluté (i).

: énergies respectivement : extérieure fournie au matériau, élastique, complémentaire (totale diminue de l'élastique), bloquée dans le matériau et en dernier lieu dissipée en chaleur.

: fraction d'énergie bloquée dans la matière.

: volume de matière.

: coefficient de sensibilité de la vitesse de déformation à la contrainte visqueuse $\left(m^{*}=1 / n^{*}\right)$.

: constantes caractérisant la fonction $K\left(R^{\vee}\right)$ affectant la composante visqueuse $\sigma_{\mathrm{v}}$.

: poids relatif de la sous-couche $(n)$.

: tenseur d'anisotropie $\left(M_{i j k l}\right)$.

: tenseur d'élasticité $\left(C_{i j k l}\right)$.

: composante anisotrope du tenseur d'anisotropie $\mathbf{M}$.

: amplitude de la restauration de la contrainte d'écoulement.

: variable d'endommagement (scalaire ou tensorielle).

Il existe d'autres constantes $\left(Q_{\mathrm{r}}, \eta^{*}, \beta^{*}, p^{(\mathrm{i})}, A^{(\mathrm{i})}, d^{*}, f^{*}, q^{*}, \ldots\right)$, qui n'apparaissent qu'une seule fois et qui sont explicitées dans le texte.

\section{Avant-propos.}

Le but du présent article de revue, partiellement présenté au Colloque Français d'Aussois 1986, "GRECO-GIS-Grandes Déformations et Endommagement », et au colloque MRES de Paris 1987 «Endommagement et Grandes déformations en Rupture à Chaud et Mise en Forme », est de faire un tour d'horizon sur les lois de comportement viscoplastique basées sur le concept thermodynamique des variables internes et étudiées en France au sein du groupe de travail «GRECO-GIS », regroupant universitaires et industriels, de spécialités différentes : métallurgistes, mécaniciens et numériciens (environ 150 personnes).

La prévision de la durée de vie d'une structure comporte essentiellement trois étapes :

- la détermination des lois de comportement du matériau utilisé, fonction de nombreux paramètres (déformation, temps, température, nature des constituants...);

- le calcul des contraintes et des déformations au sein de la structure connaissant les grandeurs qui lui sont appliquées (forces, températures...). Cette étape est généralement réalisée au moyen de calculs effectués par la technique des éléments finis qui connaît un large développement depuis une dizaine d'années ;

- la prise en compte de l'endommagement de la structure qui fait appel à la construction et à l'intégration de lois d'endommagement dans les codes de calcul, permettant de prévoir l'amorçage d'une fissure macroscopique.

Le présent article ne traite que de la première étape, c'est-à-dire des lois de comportement, et plus précisément de la classe des modèles à variables internes.

En effet, durant ces vingt dernières années, tant chez les physiciens du métal que chez les mécaniciens, les modèles rhéologiques à variables internes ont connu un large essor et sous l'impulsion des physiciens, de nombreuses techniques expérimentales pour accéder aux variables internes et à leurs lois d'évolution se sont développées. Toutes ces techniques ont été largement discutées et peuvent à présent être utilisées, à condition de bien connaitre leur domaine de validité. Pour leur part, les mécaniciens ont développé des modèles sophistiqués évoluant dans un cadre thermodynamique rigoureux mais dont la principale difficulté réside dans l'identification du nombre et de l'évolution des variables cachées introduites dans ces modèles. Ainsi, en conciliant ces deux approches, plusieurs modèles phénoménologiques quantitatifs ont été élaborés au sein de divers laboratoires universitaires et industriels, ce qui constitue le thème du présent article. On peut également signaler que les énormes progrès réalisés en informatique ont largement contribué au développement de ces lois et que certaines d'entre elles sont déjà, dans leur forme simplifiée, implantées dans des codes de calcul par éléments finis.

La présentation sera toujours intégrée, chaque fois qu'il le sera possible, dans un contexte international... c'est-à-dire qu'au cours de l'article, en faisant appel à de nombreuses références, on mettra en parallèle différents modèles et en relief les points de convergence entre des approches qui peuvent paraître a priori bien éloignées! (en particulier, celles qui ne font pas appel au concept des variables internes.) 
D'autre part, compte tenu de la fréquente complexité des modèles et pour une meilleure synthèse et compréhension de l'article, un gros effort d'unification des notations a été réalisé. Ainsi, les notations adoptées sont issues du livre «Mécanique des Matériaux Solides (1985) » [1] et par voie de conséquence les écritures originales multiples des différents auteurs ne sont pas respectées, mais, dans ce souci d'unification et de clarté, le lecteur reconnaîtra facilement le fondement d'un modèle (espace de travail), le type et la nature des variables simplement de par la nature des symboles utilisés.

\section{Introduction.}

De manière à illustrer notre propos et présenter, dans un premier temps, phénoménologiquement les problèmes liés à la modélisation du comportement des matériaux sous diverses sollicitations à hautes et moyennes températures $\left(3,3 \leqq T / T_{\mathrm{F}} \leqq 0,8\right)$, nous présentons différents résultats expérimentaux types, obtenus sur deux alliages complexes, utilisés respectivement dans les industries nucléaire et aéronautique, et très étudiés au sein du GRECO-GIS, soit l'inoxydable austénitique 17-12 SPH (du type $316 \mathrm{~L}$ ) et le superalliage base nickel Inconel 718. Comme nous le verrons ultérieurement lors de la présentation des résultats, ces deux matériaux ont des comportements très différents, voire opposés, et se situent sensiblement aux extrémités de l'échelle de la caractérisation mécanique (limite d'élasticité et ductilité à rupture lors d'un essai à vitesse imposée). Ainsi, pour qu'un modèle puisse être considéré comme universel, il devra, au moins qualitativement, être capable de restituer les différents effets observés ; ce qui est le cas des modèles présentés, du moins pour une sollicitation unidimensionnelle et pour des chargements radiaux. Par contre, pour des essais en deux dimensions hors-phase (essentiellement en traction-torsion), nous verrons la limitation de ces formulations, du moins dans leur forme actuelle, vis-à-vis des effets complexes rencontrés. $\mathrm{Ce}$ constat engendre actuellement une nouvelle phase de réflexion, d'amélioration et de développement des modèles.

S'intégrant dans un contexte thermodynamique rigoureux que nous évoquerons succinctement, les cinq modèles à variables internes présentés, tous différents dans leur approche, sont développés :

i) à l'O.N.E.R.A. (Office National d'Etudes et Recherches Aérospatiales) et au L.M.T. Cachan (Laboratoire de Mécanique et Technologie de l'ENS de Cachan et de l'Université Paris 6) ;

ii) au L.M.A.B. (Laboratoire de Mécanique Appliquée de Besançon). Les approches i) et ii) sont dites unifiées car on ne traite qu'une seule déformation viscoplastique, la plasticité classique indépen- dante du temps n'étant qu'un effet d'échelle de temps d'observation ;

iii) à l'E.M.P. (Ecole des Mines de Paris), pour une approche partitionnée où l'on additionne les déformations plastique et viscoplastique ;

iv) au C.E.A. (Commissariat à l'Energie Atomique) ;

v) au L.M.X. (Laboratoire de Mécanique des Solides de l'Ecole Polytechnique).

Les modèles iv) et $\mathrm{v}$ ) sont dits multicouches car on suppose que le volume global du matériau peut être divisé en $(n)$ sous matériaux, ou couches, associées soit en parallèle (C.E.A.), soit en série (L.M.X.). Chaque couche possède ses propres propriétés.

On présentera quelques exemples d'applications de ces modèles au cas des aciers étudiés et l'on s'efforcera de corréler le rôle de chaque terme des équations aux réalités expérimentales à modéliser (nul terme n'est innocent!). En dernier lieu, on montrera les dernières extensions de certains de ces modèles, tendant à l'amélioration de la prévision des essais hors phase. Enfin, on doit signaler que cette synthèse fait abstraction de la mécanique de l'endommagement, qui pourrait constituer, à elle seule, un article de revue [1-4].

D'autres modèles sont également étudiés dans ce groupement mais sortent du cadre des modèles à variables internes que l'on s'était fixé a priori. On peut citer, par exemple, les lois à structure héréditaire à mémoires discrètes (comportement de Masing à mémoire discrète) développées à l'I.M.G. [5] (Institut de Mécanique de Grenoble) et toute la classe des modèles locaux, partant du monocristal, c'est-à-dire de l'étude des relations entre les contraintes de cisaillement résolues sur plusieurs plans de glissement et les vitesses de cisaillement plastique résolues sur ces mêmes plans, et remontant par une formulation self-consistante à la modélisation du matériau inhomogène tel que le polycristal [6]. On peut remarquer que la notion de contraintes internes prend toute sa signification dans l'approche self-consistante (équations d'incompatibilité des déformations) et qu'un parallèle avec l'approche globale développée ci-dessous serait possible et souhaitable! On peut noter que les modèles multicouches structurels évoqués font implicitement appel aux concepts locaux puisque chaque couche est sensée représenter un grain (ou toute autre échelle locale) du polycristal.

\section{Faits expérimentaux (en une dimension : 1D).}

\subsection{PRÉSENTATION RAPIDE DES DEUX MATÉRIAUX.}

3.1.1 L'Inox. 17-12 SPH. - Il s'agit d'un acier inoxydable austénitique du type $316 \mathrm{~L}$, à très bas carbone et azote contrôlé, essentiellement utilisé dans l'industrie nucléaire et particulièrement dans la 
filière surgénérateur (Super-Phénix-E.D.F.). Le traitement thermique de la tôle consiste en un maintien de 1 h 30 à $1100{ }^{\circ} \mathrm{C}$ suivi d'une trempe à l'eau. Sa composition est la suivante :

\begin{tabular}{|c|c|c|c|c|c|c|c|c|c|c|c|c|c|c|}
\hline $\mathrm{C}$ & $\mathrm{S}$ & $\mathrm{P}$ & $\mathrm{Si}$ & $\mathrm{Mn}$ & $\mathrm{Cr}$ & $\mathrm{Ni}$ & $\mathrm{Mo}$ & $\mathrm{N}$ & $\mathrm{B}$ & $\mathrm{Co}$ & $\mathrm{Cu}$ & $\mathrm{Ti}$ & $\mathrm{Nb}$ & $\mathrm{Al}$ \\
\hline$\leqq 0,030$ & $\leqq 0,001$ & $\leqq 0,021$ & 0,44 & 1,84 & 17,31 & 12,46 & 2,47 & 0,075 & 0,001 & 0,15 & 0,175 & $\leqq 0,005$ & 0,015 & 0,100 \\
\hline
\end{tabular}

Les caractéristiques mécaniques à l'ambiante et à chaud sont :

\begin{tabular}{|c|c|c|}
\hline en $\mathrm{MPa}$ & $20^{\circ} \mathrm{C}$ & $600{ }^{\circ} \mathrm{C}$ \\
\hline$\sigma_{\mathrm{cl}}(0,2 \%)$ & 265 & 142 \\
\hline$\sigma_{\mathrm{r}}$ & 600 & 420 \\
\hline$\left(\frac{\Delta l}{l_{0}}\right)_{\mathrm{r}}$ & $60 \%$ & $48 \%$ \\
\hline
\end{tabular}

$\sigma_{\mathrm{cl}}(0,2 \%) \quad$ est la limite élastique

$\sigma_{\mathrm{r}} \quad$ la contrainte à la rupture et

$\left(\frac{\Delta l}{l_{0}}\right)$

la ductilité à rupture.

3.1.2 L'Inco 718. - Il s'agit d'un superalliage base nickel dont la composition est reportée ci-dessous et principalement utilisé pour la construction de disques porte-aubes de turbomachines (S.N.E.C.M.A.).

\begin{tabular}{|c|c|c|c|c|c|c|c|c|c|c|c|}
\hline $\mathrm{Ni}$ & $\mathrm{Cr}$ & $\mathrm{Fe}$ & $\mathrm{Nb}$ & $\mathrm{Ti}$ & $\mathrm{Al}$ & $\mathrm{Mo}$ & $\mathrm{C}$ & $\mathrm{Bi}$ & $\mathrm{Pb}$ & $\mathrm{Ag}$ & $\mathrm{Sn}$ \\
\hline 52,2 & 18,26 & 18,38 & 4,67 & 1,08 & 0,55 & 2,98 & 0,065 & $\leqq 0,5$ & $\leqq 1$ & $\leqq 1$ & 60 \\
\hline
\end{tabular}

Les éprouvettes sont prélevées dans des barres traitées thermiquement de la manière suivante; mise en solution à $950^{\circ} \mathrm{C}$ pendant une heure, refroidissement à l'air, revenu à $720^{\circ} \mathrm{C}$ pendant $8 \mathrm{~h}$,refroidissement jusqu'à $620^{\circ} \mathrm{C}$ suivi d'un maintien pendant $8 \mathrm{~h}$ puis d'une trempe à l'eau. Le durcissement est ainsi obtenu par précipitation structurale des phases $\gamma^{\prime}$ et $\gamma^{\prime \prime}$. Les propriétés mécaniques résultantes sont :

\begin{tabular}{|c|c|c|}
\hline en $\mathrm{MPa}$ & $20{ }^{\circ} \mathrm{C}$ & $650{ }^{\circ} \mathrm{C}$ \\
\hline$\sigma_{\mathrm{cl}}(0,2 \%)$ & 1055 & 910 \\
\hline$\sigma_{\mathrm{r}}$ & 1350 & 1150 \\
\hline$\left(\frac{\Delta l}{l_{0}}\right)_{\mathrm{r}}$ & \multicolumn{2}{|c}{$\simeq 20 \%$} \\
\hline
\end{tabular}

\subsection{PROPRIÉTÉS MONOTONE ET CYCLIQUE.}

3.2.1 Courbes monotones. - Sur la figure 1, on reporte pour les deux alliages le début des courbes de traction monotone à vitesse imposée, $\dot{\varepsilon}^{\mathrm{T}} \simeq$ $6 \times 10^{-4} \mathrm{~S}^{-1}$, et sensiblement à la même température, $T \simeq 600^{\circ} \mathrm{C}$. On visualise ainsi l'éloignement de leurs caractéristiques mécaniques, à savoir : très haute limite élastique conventionnelle pour l'INCO $\left(\sigma_{\mathrm{el}}(0,2 \%) \simeq 995 \mathrm{MPa}\right.$ à $\left.600^{\circ} \mathrm{C}\right)$ associée à une assez faible ductilité $\left(\left(\frac{\Delta l}{l_{0}}\right)_{\mathrm{r}} \simeq 20 \%\right)$ et à l'opposé, une très faible limite élastique et une grande ductilité pour le 17-12 SPH $\left(\sigma_{\mathrm{el}}(0,2) \simeq\right.$ $140 \mathrm{MPa}$ et $\left(\frac{\Delta l}{l_{0}}\right)_{\mathrm{r}} \simeq 50 \%$ à $\left.600^{\circ} \mathrm{C}\right)$.

D'une façon similaire, sur la figure 2 , on reporte quelques courbes de fluage jusqu'à rupture pour des contraintes situées sur la figure 1 . Comme précédem-

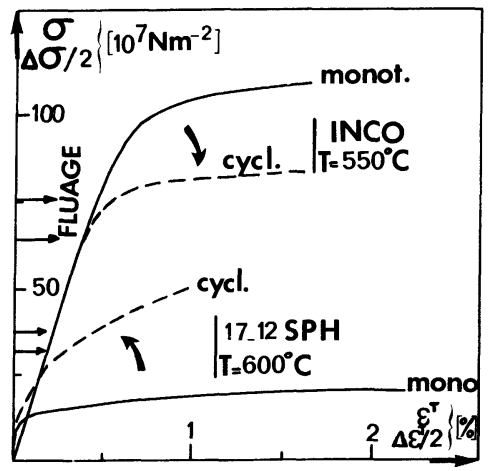

Fig. 1. - Courbes d'écrouissage monotone et cyclique de l'INCO 718 et du 17-12 SPH, respectivement à 550 et $600{ }^{\circ} \mathrm{C}$. Mise en évidence de la déconsolidation cyclique dans le premier cas et de la consolidation cyclique dans le second. (D'après De Langre et Rousset [15] et Nouailhas [17], avec l'amabilité du GIS « Rupture à Chaud » et de l'ONERA.)

[Monotonic and cyclic hardening curves of INCO 718 and $17-12 \mathrm{SPH}$ respectively at 550 and $600{ }^{\circ} \mathrm{C}$. Cyclic softening in the first material and cyclic hardening in the other one are pointed out. (From De Langre and Rousset [15] and Nouailhas [17], with courtesy of the GIS « Rupture à Chaud » and ONERA.).] 


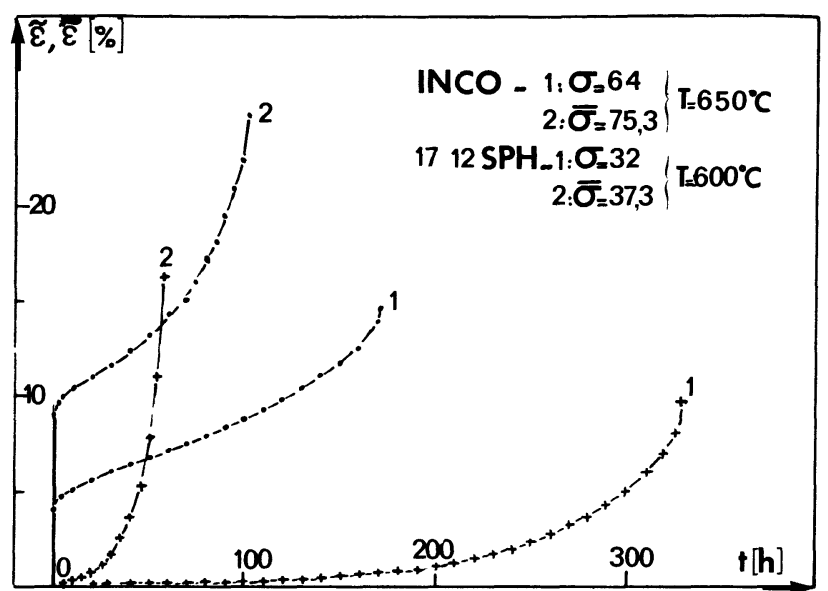

Fig. 2. - Fluage de l'INCO 718 et du 17-12 SPH, à contrainte constante: INCO : $T=650{ }^{\circ} \mathrm{C}$, (1) $\sigma=640 \mathrm{MPa}$, (2) $\quad \sigma=753 \mathrm{MPa} ; \quad 17-12 \mathrm{SPH}$ : $T=600{ }^{\circ} \mathrm{C}$, (1) $\sigma=320 \mathrm{MPa}$, (2) $\sigma=373 \mathrm{MPa}$. (D'après Delobelle [7, 9], avec l'amabilité du J. Nucl. Mater.)

[Constant stress creep of INCO 718 and 17-12 SPH : INCO : $T=650^{\circ} \mathrm{C}$, (1) $\sigma=640 \mathrm{MPa}$, (2) $\sigma=$ $753 \mathrm{MPa} ; 17-12 \mathrm{SPH}: T=600{ }^{\circ} \mathrm{C}$, (1) $\sigma=320 \mathrm{MPa}$, (2) $\sigma=373 \mathrm{MPa}$. (From Delobelle [7, 9], with courtesy of $J$. Nucl. Mater.).]

ment, leur aspect est très différent : faible fluage primaire immédiatement suivi d'un stade tertiaire très développé pour l'INCO $(90 \%$ de la durée de vie pour une contrainte axiale $\sigma_{z z}=640 \mathrm{MPa}$ [7]), alors que pour l'inoxydable, il apparaît une très forte plasticité durant la mise en charge, (de l'ordre de $4 \%$ pour $\sigma_{z z}=320 \mathrm{MPa}$ [7]) correspondant sensiblement à la courbe effort déformation [7-8]. La mise en charge terminée, on assiste à une phase de consolidation primaire bien développée, suivie d'un stade quasi-stationnaire débouchant sur la période d'endommagement correspondant à la phase tertiaire conduisant à la rupture. Dans ce dernier cas, on comprend les approches (i), (ii) et (iii) qui analysent respectivement la déformation «instantanée » de chargement comme viscoplastique (i) et (ii), ou plastique (iii). Ainsi, la déformation totale de fluage $\varepsilon^{T}$ est la somme de deux déformations $\varepsilon^{\mathrm{cl}}$ et $\varepsilon^{\mathrm{v}}$ dans les approches unifiées (i) et (ii) et de trois dans l'approche partitionnée (iii) ; élastique, plastique et viscoplastique $\left(\varepsilon^{\mathrm{el}}, \varepsilon^{\mathrm{p}}\right.$ et $\left.\varepsilon^{\eta}\right)$, soit :

$$
\left.\begin{array}{ll}
\varepsilon^{\mathrm{T}}=\varepsilon^{\mathrm{cl}}+\varepsilon^{\mathrm{v}} & \text { (i) et (ii) } \\
\varepsilon^{\mathrm{T}}=\varepsilon^{\mathrm{cl}}+\varepsilon^{\mathrm{p}}+\varepsilon^{\mathrm{v}} & \text { (iii) }
\end{array}\right\} .
$$

Notons que la seconde solution est très souvent utilisée puisqu'elle consiste simplement à additionner les lois de la plasticité indépendante du temps aux lois viscoplastiques dépendant du temps et ceci, indépendamment des lois choisies pour chaque type de déformation. Par contre, avec la formulation unifiée, la plasticité apparaît comme une solution asymptotique de la viscoplasticité lorsque l'intervalle de temps considéré tend vers zéro. Pour le 17$12 \mathrm{SPH}$, la quasi-insensibilité des courbes effortsdéformations à la vitesse de sollicitation, du moins dans l'intervalle $350 \leqq T \leqq 550^{\circ} \mathrm{C}$ et $3,3 \times 10^{-4} \leqq$ $\dot{\varepsilon}^{\mathrm{T}} \leqq 1,3 \times 10^{-2} \mathrm{~S}^{-1},[7,9]$, tend à démontrer la nature plastique de la déformation et accrédite l'idée première de la partition de la déformation.

Par contre, à plus haute température, l'agitation thermique aidant, la composante viscoplastique

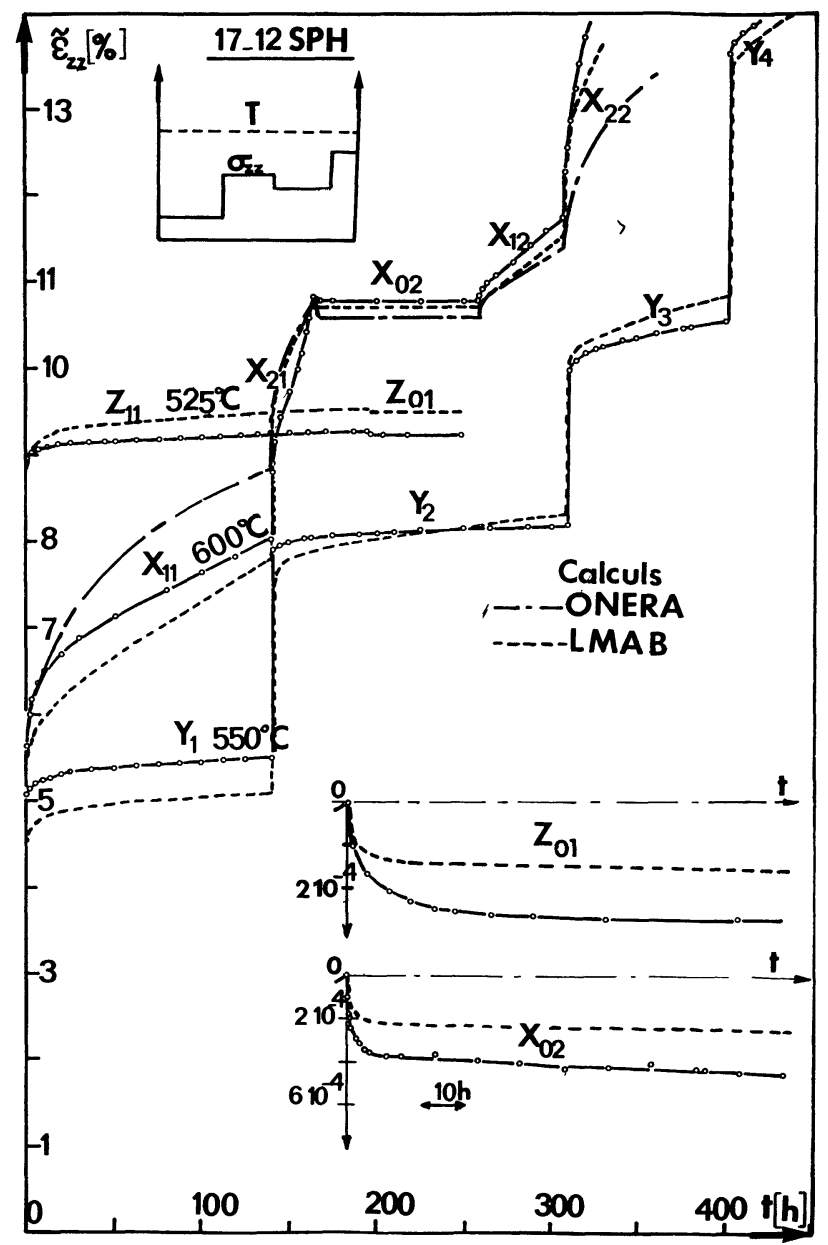

Fig. 3. - Acier inoxydable 17-12 SPH. Fluage sous différents niveaux de contrainte, mise en évidence de la plasticité à chaque incrément de contrainte et de la recouvrance pour $\sigma=0 . \otimes T=600^{\circ} \mathrm{C}, \quad X_{11}=$ $286 \mathrm{MPa}, \quad X_{21}=323 \mathrm{MPa}, X_{02}=0, X_{12}=229 \mathrm{MPa}$ et $X_{22}=331 \mathrm{MPa}$; (Y) $T=550{ }^{\circ} \mathrm{C}, Y_{1}=271 \mathrm{MPa}, Y_{2}=$ $325 \mathrm{MPa}, \quad Y_{3}=362 \mathrm{MPa}$ et $Y_{4}=408 \mathrm{MPa}$; (Z) $T=$ $525^{\circ} \mathrm{C}, Z_{11}=347 \mathrm{MPa}$ et $Z_{01}=0$. (D'après Delobelle [7], thèse, France).

[17-12 SPH Stainless steel. Creep at various stress levels. The plastic strains at each stress increment and the recovery at $\sigma=0$ is pointed out: Q $T=600^{\circ} \mathrm{C}$, $X_{11}=286 \mathrm{MPa}, X_{21}=323 \mathrm{MPa}, X_{02}=0, X_{12}=229 \mathrm{MPa}$ et $X_{22}=331 \mathrm{MPa}$. (Y) $T=550^{\circ} \mathrm{C}, Y_{1}=271 \mathrm{MPa}, Y_{2}=$ $325 \mathrm{MPa}, Y_{3}=362 \mathrm{MPa}$ et $Y_{4}=408 \mathrm{MPa}$. (Z) $T=$ $525^{\circ} \mathrm{C}, Z_{11}=347 \mathrm{MPa}$ et $Z_{01}=0$. (From Delobelle [7], thesis, France).] 
n'est plus négligeable et la contrainte d'écoulement dépend naturellement de la vitesse de déformation. Cependant, sur une courbe de fluage, telle que celle de la figure 2, on visualise l'arbitraire d'une telle décomposition, la notion d'échelle de temps d'observation étant intrinsèquement liée à la définition de la déformation dite indépendante du temps.

Ce problème est moins aigu sur l'INCO qui ne présente pas de grande déformation quasiment indépendante du temps et une approche purement visqueuse est généralement admise, ce qui rejoint la formulation unifiée et partitionnée avec $\varepsilon^{p}=0$.

Les conditions d'apparition de la «plasticité » et de l'éventuel couplage avec le fluage peuvent être mis en évidence par des essais de fluage à plusieurs niveaux à contrainte croissante ou décroissante (Fig. 3). Ainsi, on montre qu'à chaque incrément de contrainte correspond un incrément de «plasticité » suivi d'une période de fluage dont la vitesse est d'autant plus rapide que la contrainte et la température sont élevées. Par contre, si l'on réalise une décharge totale, on enregistre une déformation négative de recouvrance de faible amplitude (quelques $10^{-4}$ ) croissante avec la température et fonction de l'état de déformation préalable [7, 10]. Si l'on retourne ensuite à la valeur nominale de la contrainte, suivant la température de l'essai et le temps passé à contrainte nulle, il peut se manifester une composante "plastique » au rechargement [7]. Cette constatation expérimentale tend à montrer que plasticité et fluage sont deux phénomènes liés. Cette interaction est également visible sur la figure 4 où l'on compare une courbe de traction monotone $\mathrm{OC}$ avec une réponse en traction-fluage-traction OABC" [11]. Si fluage et plasticité n'étaient pas liés, on obtiendrait une courbe telle que $\mathrm{BC}^{\prime}$, ce qui n'est

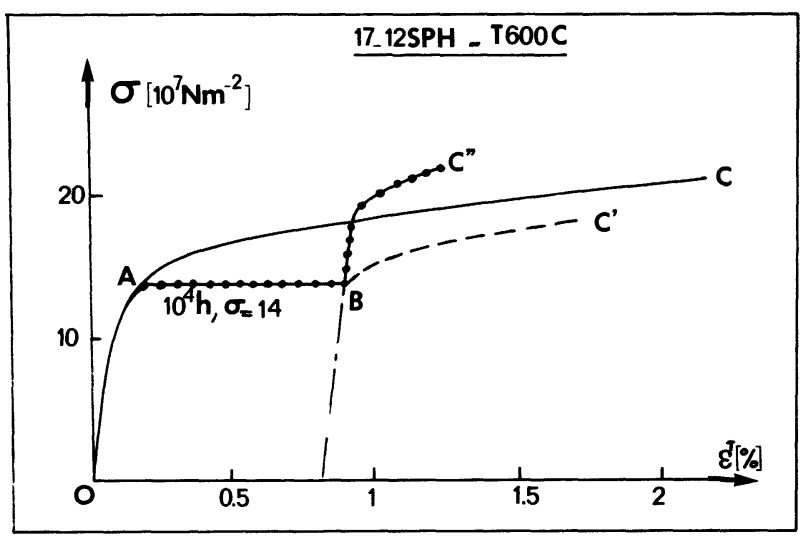

Fig. 4. - Acier inoxydable 17-12 SPH, $T=600^{\circ} \mathrm{C}, \sigma=$ $140 \mathrm{MPa}$. Mise en évidence de l'interaction plasticitéfluage (vieillissement). (D'après Crouzet [11], avec l'amabilité d'E.D.F.).

[Stainless steel $17-12 \mathrm{SPH}, T=600^{\circ} \mathrm{C}, \quad \sigma=140 \mathrm{MPa}$. Demonstration of the interaction of creep and plasticity (ageing). (From Crouzet [11] with courtesy of E.D.F.).] pas, de toute évidence, le cas de $\mathrm{BC}^{\prime \prime}$. La branche $\mathrm{BC}^{\prime \prime}$ est même supérieure à la courbe monotone $\mathrm{OC}$. Cependant, compte tenu du temps de maintien en fluage, il peut également apparaître des phénomènes de vieillissement structural. On peut noter qu'une telle observation avait déjà été mentionnée par Pugh [12]. Ces deux exemples montrent clairement que le fluage influence la composante «plastique ».

A l'inverse, si l'on réalise des essais de fluage au même niveau de contrainte mais après différentes histoires de chargement [7, 13] (Fig. 5: fluage directement sous $\sigma_{1}$ ou après un incrément à vitesse imposée jusqu'à $\sigma_{2}$ suivi d'une période de fluage sous $\sigma_{1}[13]$ ) on constate qu'après des transitoires de concavités inverses, l'état quasi-stationnaire est identique dans les deux cas. Ainsi, la plasticité (échelle de temps réduite) semble ne pas avoir d'effet évident sur le fluage à long terme.

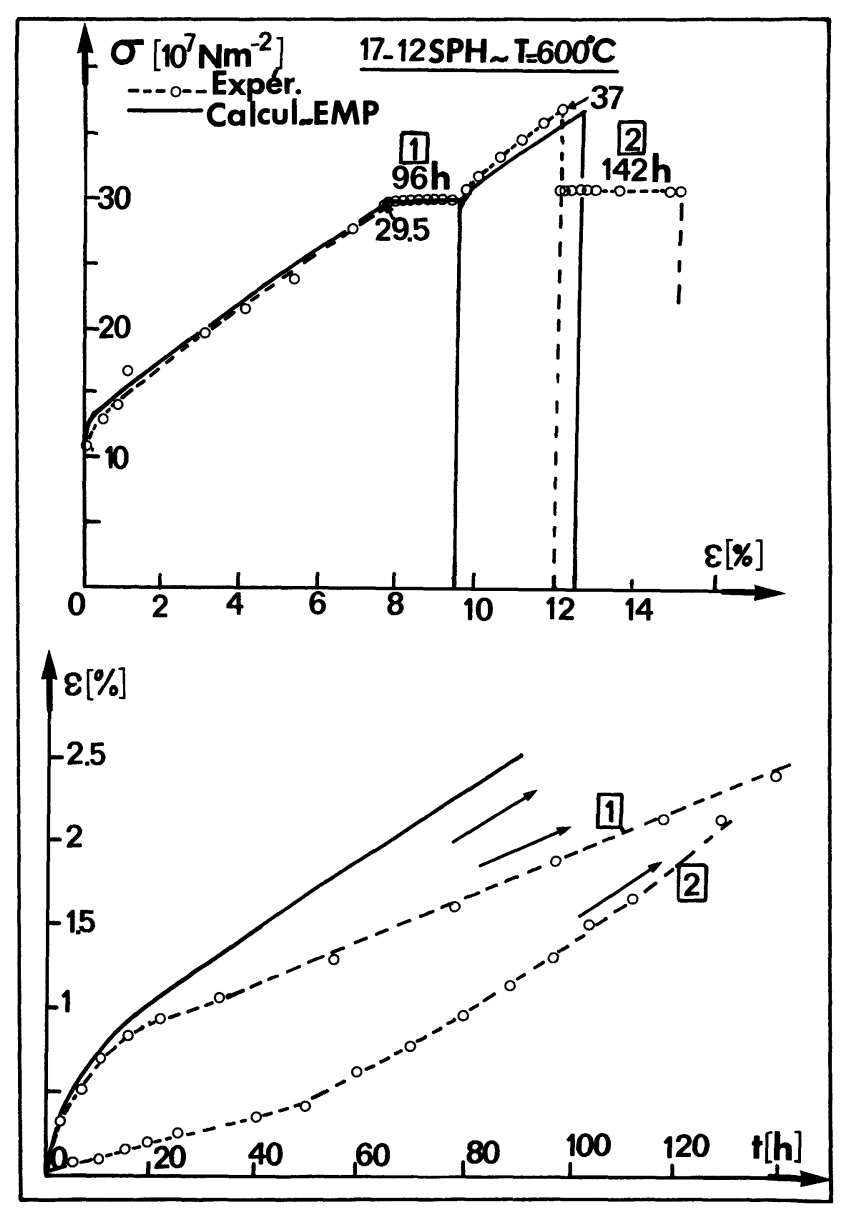

Fig. 5. - Fluage du 17-12 SPH au même niveau de contrainte mais après deux histoires de chargement différentes: indifférence de l'état stationnaire à l'histoire antérieure. (D'après Contesti [13], avec l'amabilité de l'EMP et du GIS « Rupture à Chaud»).

[Creep of 17-12 SPH at a given stress level but for two different loading paths : independancy of steady creep versus the previous history. (From Contesti [13], with courtesy from EMP and the GIS « Rupture à Chaud»).] 
En conclusion, de ces quelques aspects expérimentaux, on en déduit que plasticité et fluage sont toujours des phénomènes couplés, ce qui abonde dans le sens des théories unifiées. Cependant, dans les théories partitionnées, le couplage peut s'effectuer au niveau des lois d'évolution des variables internes. Succinctement, en ce qui concerne les modèles multicouches, ce couplage peut être restitué, à condition d'avoir un nombre de couches suffisant pour pouvoir balayer un spectre de constantes, affectées à chaque couche, assez large.

3.2.2 Propriétés cycliques. - Sur les figures 6 [14] et 7 [15], respectivement pour le 17-12 SPH et l'INCO, on reporte quelques cycles obtenus par cyclage à vitesse imposée $\left( \pm \dot{\varepsilon}^{\mathrm{T}}\right)$ entre déformations symétriques également imposées. Alors que l'inoxydable consolide fortement au cours du cyclage jusqu'à atteindre sa valeur stabilisée, à l'opposé, l'INCO se déconsolide lentement. Les courbes cycliques des valeurs à saturation $\left(\frac{\Delta \sigma}{2}\right)$ en fonction de l'ampli-

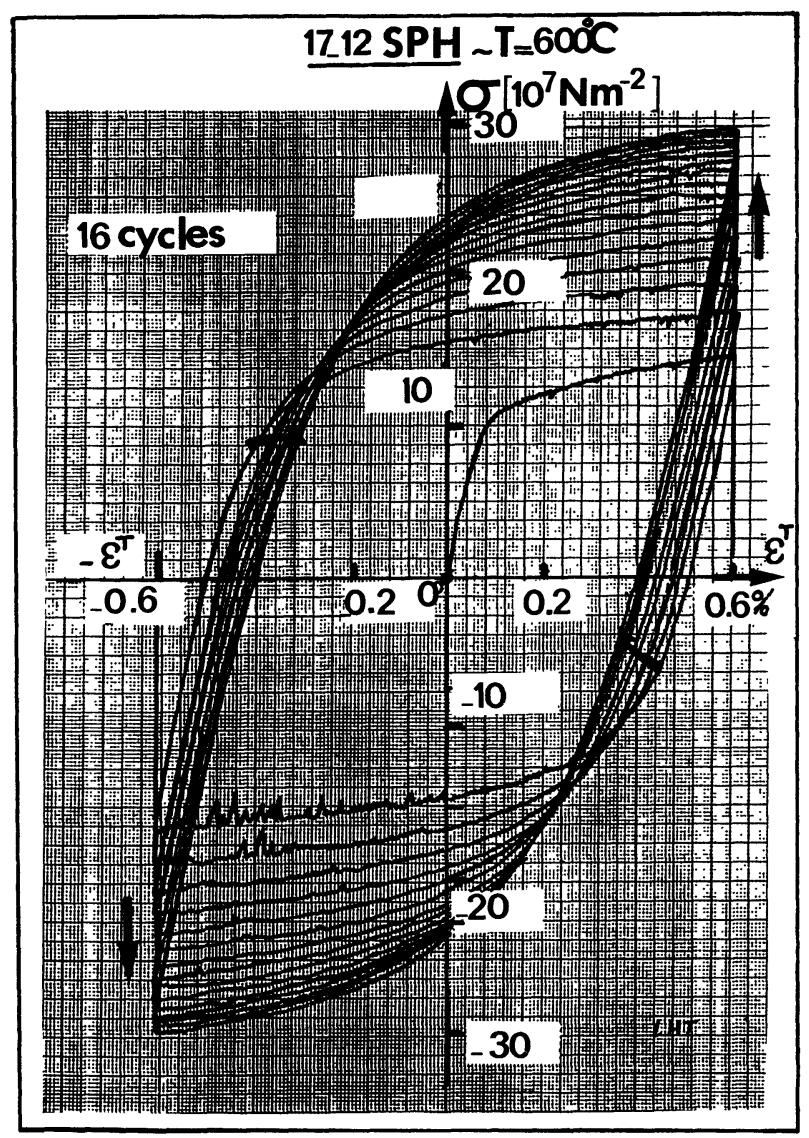

Fig. 6. - Consolidation cyclique d'un acier 17-12 SPH à $T=600{ }^{\circ} \mathrm{C}$ et $\Delta \varepsilon^{\mathrm{T}}= \pm 0,6 \% \quad(16$ premiers cycles $)$. (D'après Marquis [14], avec l'amabilité du GIS « Rupture à Chaud »).

[Cyclic hardening of 17-12 SPH at $T=600{ }^{\circ} \mathrm{C}$ and $\Delta \varepsilon^{\mathrm{T}}=$ $\pm 0.6 \%$ (16 first cycles). (From Marquis [14] with courtesy of the GIS «Rupture à Chaud»).]

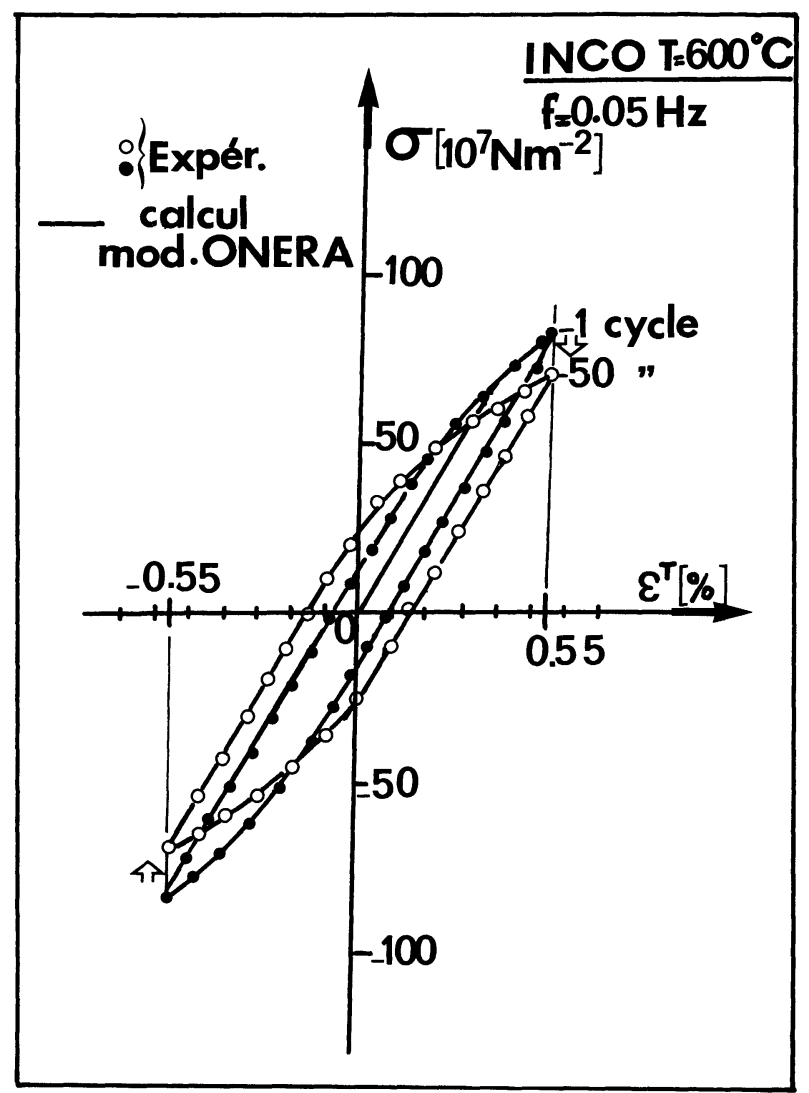

Fig. 7. - Déconsolidation cyclique de l'INCO 718 à $T=600{ }^{\circ} \mathrm{C}$ et $\Delta \varepsilon^{\mathrm{T}}= \pm 0,55 \% \quad(50$ premiers cycles $)$. (D'après De Langre et Rousset [15], DEA au LMX).

[Cyclic softening of INCO 718 at $T=600{ }^{\circ} \mathrm{C}$ and $\Delta \varepsilon^{\mathrm{T}}=$ $\pm 0.55 \%$ (50 first cycles).(From De Langre and Rousset [15], DEA at LMX).]

tude de déformation totale au cycle stabilisé $\left(\frac{\Delta \varepsilon^{\mathrm{T}}}{2}\right)$ sont reportées sur la figure $1,\left(\frac{\Delta \sigma}{2}=\right.$ $\left.f\left(\frac{\Delta \varepsilon^{T}}{2}\right)\right)$ et peuvent être comparées aux courbes monotones. Pour l'INCO qui présente une très forte limite élastique initiale, la courbe cyclique se situe en dessous de la courbe monotone (déconsolidation) alors que pour le 17-12 SPH qui possède des faibles propriétés monotones, la courbe cyclique est fortement au-dessus (consolidation).

De plus, si l'on observe de près les cycles effortsdéformations, on s'aperçoit que le module plastique tangent à chaque inversion augmente avec la consolidation dans le cas de l'inoxydable et a tendance à diminuer dans le cas du superalliage qui déconsolide. D'autre part, on peut noter que la plastification (perte de linéarité dans la réponse $\sigma=f(\varepsilon)$ ) lors d'une inversion, indépendamment du cycle considéré, se situe toujours au voisinage de l'axe $\sigma_{z z}=0$. Nous verrons par la suite comment ces différents aspects de comportement peuvent être intégrés dans les modèles. 
Comme le montre la figure 8 [11], à l'instar des essais monotones évoqués précédemment, on peut à nouveau mettre clairement en évidence le couplage entre plasticité et fluage en réalisant plusieurs essais de fluage pour la même contrainte, après consolidation cyclique, mais sur les deux branches opposées d'un même cycle. Les deux courbes ainsi obtenues sont différentes et se situent en dessous de celle observée directement après une mise en charge monotone.

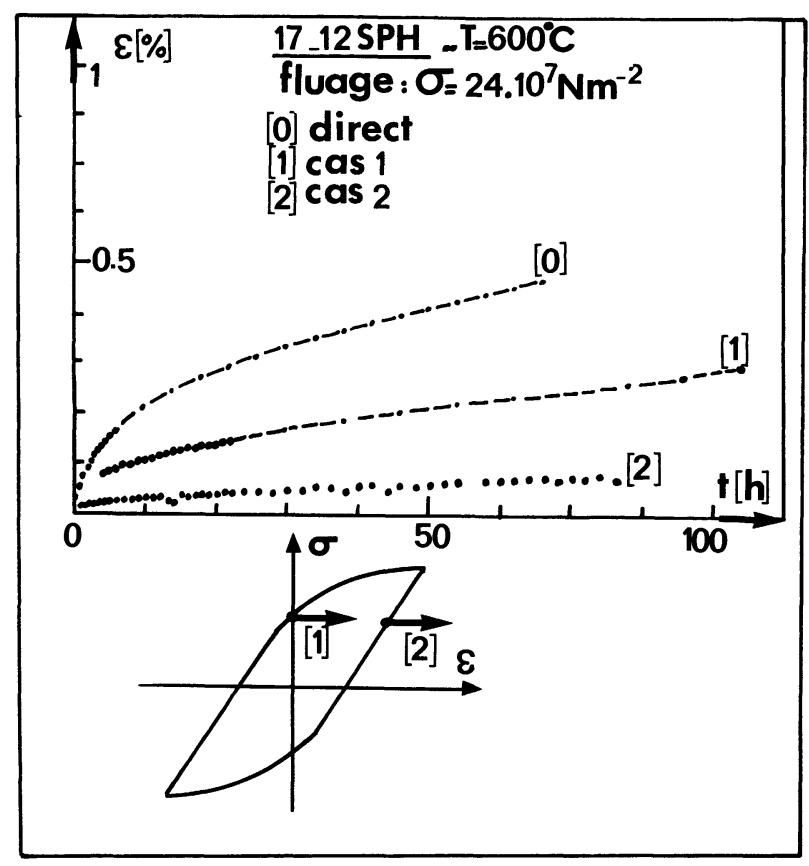

Fig. 8. - Influence d'un écrouissage cyclique sur le fluage ultérieur. (D'après Crouzet [11], avec l'amabilité d'E.D.F.).

[Role of a cyclic hardening on the consecutive creep. (From Crouzet [11], with courtesy of E.D.F.).]

Un autre phénomène cyclique présent sur quelques matériaux et particulièrement sur le 17-12 SPH est l'effet de mémoire de la prédéformation plastique maximale (Fig. 9). Ainsi, après saturation de l'écrouissage cyclique pour un niveau de déformation $\Delta \varepsilon_{1}^{\mathrm{T}}$, si l'on augmente l'amplitude de cyclage à $\Delta \varepsilon_{2}^{\mathrm{T}}$, il apparaît une nouvelle consolidation cyclique jusqu'à la nouvelle saturation correspondant à $\Delta \varepsilon_{2}^{\mathrm{T}}$ et ainsi de suite pour des niveaux croissants de déformation. Par contre, si l'on retourne au plus faible niveau de déformation $\Delta \varepsilon_{1}^{\mathrm{T}}$, la valeur stabilisée du cycle correspond sensiblement à celle obtenue lors du précyclage sous la plus grande amplitude. Ceci correspond à la mise en mémoire de la prédéformation maximale [16]. Cependant, dans certaines conditions de très forts préécrouissages, cet effet est partiellement évanescent, c'est-à-dire qu'au cours du

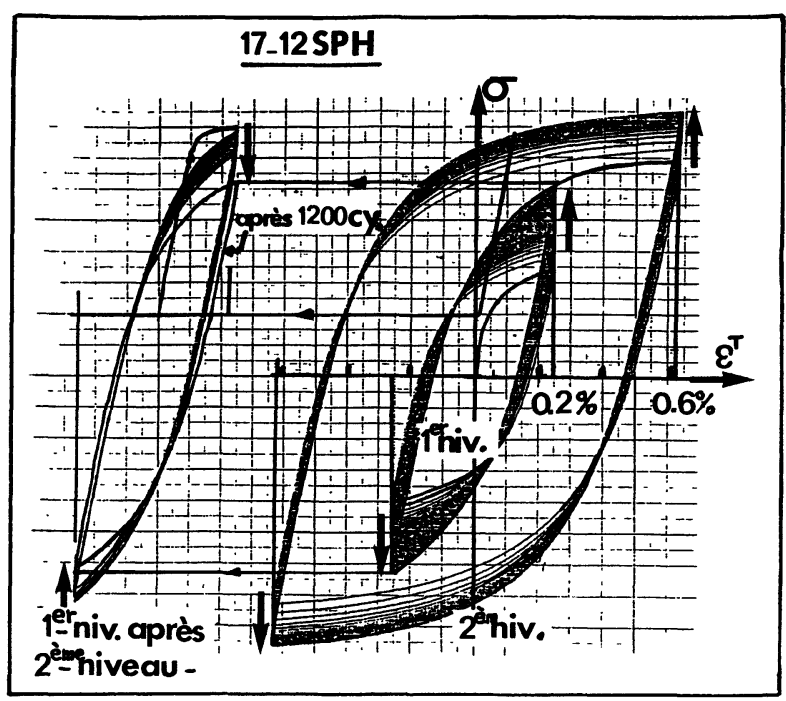

Fig. 9. - Inoxydable 17-12 SPH. Effet de mémoire de la prédéformation plastique maximale. Cyclage à $\Delta \varepsilon^{\mathrm{T} 1}=$ $\pm 0,2 \%$ puis $\Delta \varepsilon^{\mathrm{T} 2}= \pm 0,65 \%$ et retour à $\Delta \varepsilon^{\mathrm{T} 1}= \pm 0,2 \%$. (D'après Marquis [14], avec l'amabilité du GIS « Rupture à Chaud »).

[Stainless steel 17-12 SPH. After effect of the maximum plastic strain. Cyclic test with $\Delta \varepsilon^{\mathrm{T}_{1}}= \pm 0.2 \%$ then $\Delta \varepsilon^{\mathrm{T}_{2}}= \pm 0.65 \%$ and back again to $\Delta \varepsilon^{\mathrm{T}_{1}}= \pm 0.2 \%$. (From Marquis [14] with courtesy of the GIS « Rupture à Chaud»).]

cyclage sous le plus faible niveau, la valeur stabilisée du cycle a tendance à décroître à nouveau, pour éventuellement retrouver la valeur correspondant au cycle stabilisé obtenu directement pour la plus faible amplitude.

3.2.3 Effets du temps. - Ces effets sont les conséquences de la restauration avec le temps des phénomènes d'écrouissages induits par la déformation et sont d'autant plus importants que la température est élevée, c'est-à-dire que l'agitation thermique est efficace.

Ainsi, pour un essai de fluage à haute température, le stade stationnaire $\left(\dot{\varepsilon}^{\text {st }}=\right.$ Cte) est la conséquence de l'équilibre entre écrouissage par la déformation et restauration par le temps. De même, durant un essai à vitesse imposée (Fig. 10), si l'on décharge totalement l'échantillon pendant un temps $t_{\mathrm{m}}$, la nouvelle limite élastique apparente obtenue lors de la remise en charge est inférieure à la contrainte d'écoulement avant décharge. La partie restaurée de la contrainte est quantifiable par le paramètre $\Delta \sigma_{\mathrm{r}}$ et l'on peut montrer que $\Delta \sigma_{\mathrm{r}}$ est une fonction croissante de la température, de la déformation et bien évidemment du temps de maintien $t_{\mathrm{m}}$ (Fig. 10) [7, 9]. On obtient le même phénomène après consolidation cyclique suivi d'un vieillissement à contrainte nulle puis d'une remise en charge à vitesse imposée (Fig. 11) [17]. 


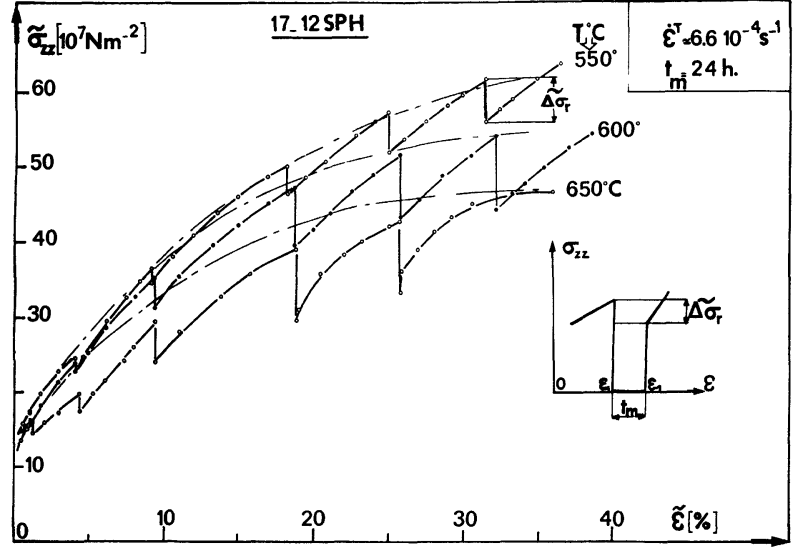

Fig. 10. - Inoxydable 17-12 SPH : influence d'un temps de maintien de $24 \mathrm{~h}$ à contrainte nulle sur les courbes effort-déformation à $T=550,600$ et $650^{\circ} \mathrm{C}$. (D'après Delobelle [7], thèse, France).

[17-12 SPH stainless steel : effect of a 24 hours holding time at zero stress on the stress strain curves at $T=$ 550, 600, $650{ }^{\circ} \mathrm{C}$. (From Delobelle [7], thesis, France).]

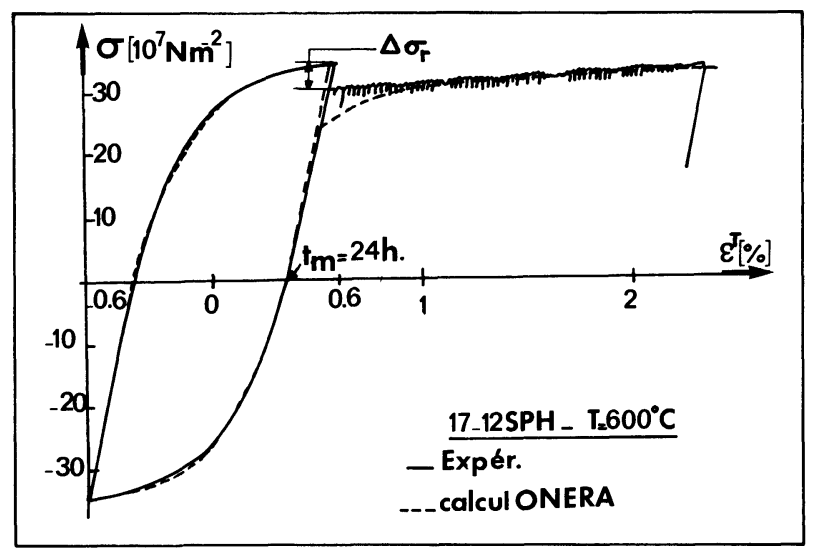

Fig. 11. - Inoxydable 17-12 SPH : influence d'un maintien de $24 \mathrm{~h}$ à force nulle après consolidation cyclique à $T=660^{\circ} \mathrm{C}$. (D'après Nouailhas [17], avec l'amabilité de l'ONERA).

[17-12 SPH stainless steel - effect of a 24 hours holding time at zero stress after cyclic hardening at $T=660{ }^{\circ} \mathrm{C}$. (From Nouailhas [17], with courtesy of ONERA).]

Une étude systématique de l'influence d'un temps de maintien $t_{\mathrm{m}}$, au maximum de déformation, sur la courbe de consolidation cyclique à $600{ }^{\circ} \mathrm{C}$ de l'acier 17-12 SPH illustre parfaitement les conséquences importantes de la restauration et corrobore les résultats précités, à savoir que la courbe cyclique est d'autant plus basse que la déformation et le temps de maintien sont élevés (Fig. 12). Ces différentes manifestations du temps devront, bien évidemment, être prises en compte lors de la modélisation.

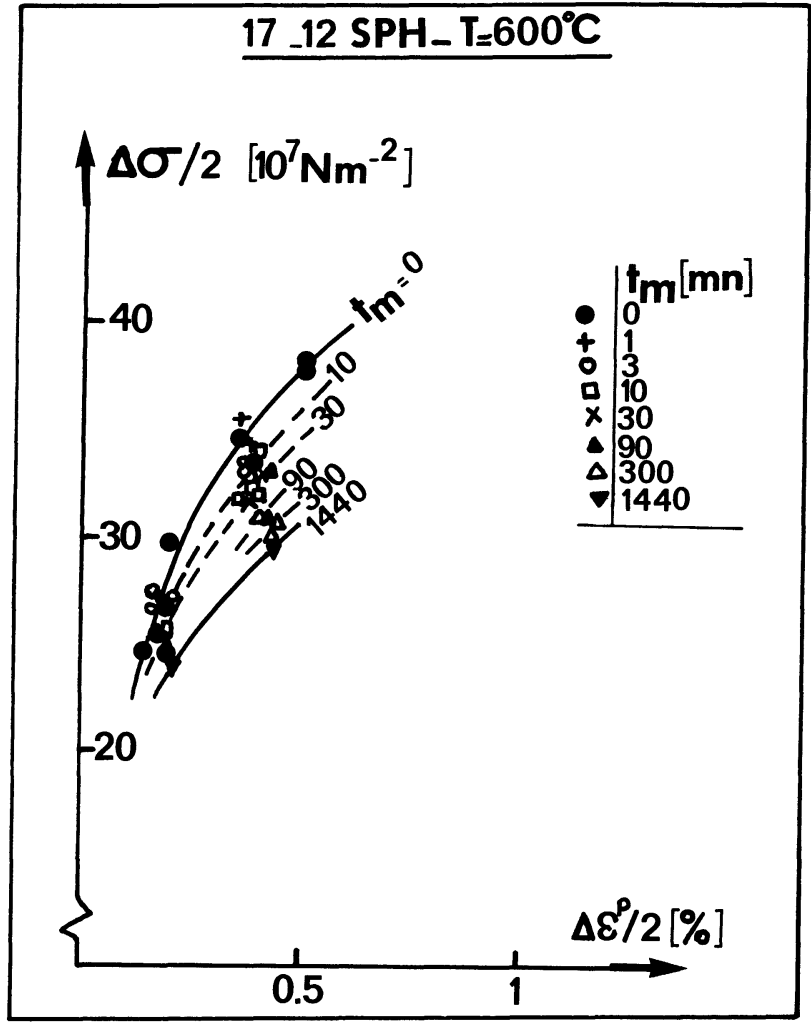

Fig. 12. - Acier inoxydable : influence d'un temps de maintien $t_{\mathrm{m}}$ sur la courbe de consolidation cyclique à $T=600^{\circ} \mathrm{C}$. (D'après Nouailhas [17], avec l'amabilité de l'ONERA).

[Stainless steel : influence of an holding time on the cyclic hardening curve at $T=600^{\circ} \mathrm{C}$. (From Nouailhas [17], with courtesy of ONERA).]

\section{Cadre thermodynamique d'évolution de ces} modèles.

4.1 CADRE THERMOdYNAMIQUE GÉNÉRAL. L'ensemble des modèles à variables internes présentés s'insèrent dans un cadre thermodynamique des processus irréversibles dans les milieux continus, bien précis, que nous évoquons brièvement. Pour un développement plus détaillé, se référer aux articles généraux [1, 18-21].

Avant tout propos, il faut mentionner que l'ensemble des développements présentés se situent toujours dans le cadre des petites déformations (hypothèse des transformations infinitésimales), ce qui revient à confondre les variables eulérienne et lagrangienne respectivement repérées par rapport aux configurations actuelle et initiale, c'est-à-dire de négliger les termes du second ordre permettant le passage entre les deux formulations. Cette simplification permet de réaliser de façon additive la décomposition de la déformation viscoplastique totale en déformations élastique et anélastique (Eq. (1)), ce qui ne peut être réalisé en grande déformation qu'en introduisant une configuration relâchée et en redéfinissant des 
nouveaux tenseurs de déformation et de contrainte, l'ensemble conduisant à un formalisme assez complexe [19, 20, 30].

En petite déformation, l'approche repose sur la méthode de l'état local et consiste à appliquer les concepts de la thermostatique classique à un petit élément matériel déformé plastiquement, cet élément étant considéré comme un système thermodynamique en « équilibre contraint ». Ainsi, on postule que l'équilibre est assuré non seulement par les contraintes externes mais également par les contraintes internes (fictives ou réelles) et l'on suppose que l'évolution du milieu peut être considéré comme une succession d'états d'équilibre thermodynamiquement admissibles, c'est-à-dire qu'il existe un potentiel thermodynamique, l'énergie libre par exemple, défini à partir des variables d'état prises en compte et qui permet de décrire l'état du système à un instant donné. A ce stade, on peut noter que les variables internes de nature scalaire ou tensorielle représentent l'état actuel de la matière, c'est-à-dire l'état d'écrouissage interne de la matière, et que le choix arbitraire de la nature et du nombre des variables permet de décrire plus ou moins bien les différents phénomènes physiques considérés.

Ainsi, si $\rho, U, S, T, \sigma_{i j}, \dot{\varepsilon}_{i j}$ et $\mathbf{q}$ sont respectivement la masse volumique, l'énergie interne spécifique, l'entropie, la température de l'élément de volume, les composantes du tenseur de contrainte de Cauchy, les composantes du tenseur de vitesse de déformation et le flux de chaleur, la combinaison de l'équation de conservation de l'énergie (premier principe) et la définition de l'énergie libre spécifique $\psi$, permet de traduire le second principe de la thermodynamique (vitesse de production d'entropie interne positive) sous la forme de l'inégalité de Clausius-Duhem :

$$
\sigma_{i j} \dot{\varepsilon}_{i j}-\rho(\psi+\dot{T} S)-\frac{1}{T} \mathbf{q} \operatorname{grad} T \geqq 0 .
$$

En faisant apparaître la dissipation intrinsèque $D$, (2) s'écrit :

$$
\rho D-\frac{1}{T} \mathbf{q} \operatorname{grad} T \geqq 0
$$

On suppose qu'il y a découplage entre dissipations intrinsèque et thermique, c'est-à-dire que (3) peut se décomposer selon $D \geqq 0$ et $\frac{1}{T} \mathbf{q} \operatorname{grad} T \leqq 0$. La seconde inégalité est vérifiée par les lois de conduction de la chaleur de Fourier.

D'une manière générale, on peut exprimer $\dot{\psi}$ en fonction des dérivées particulaires des variables d'états du matériau et l'inégalité (3) se met sous la forme :

$$
D=\sum_{k=1}^{n} A^{k} \dot{a}^{k} \geqq 0
$$

Les $A^{k}$ sont les forces thermodynamiques généralisées fonctions des variables d'état et $\dot{a}^{k}$ les flux thermodynamiques. A ce point, il est fondamental de remarquer qu'en plus de l'inégalité (4) traduisant la positivité de la dissipation, il faut définir les lois qui gouvernent la déformation et les variables cachées ; le nombre de ces variables étant, rappelons-le, un compromis entre la complexité du modèle et la fidélité de la description des phénomènes.

Une démarche pratique pour vérifier a priori l'inégalité (4) est d'évoluer dans les concepts de la thermodynamique des processus irréversibles et d'étendre les relations de réciprocité d'Onsager du cas linéaire au cas non linéaire par l'introduction d'un potentiel de dissipation $\omega$, ce qui conduit à appliquer l'hypothèse de normalité généralisée qui correspond à une extension à l'ensemble des variables internes du principe du travail maximum de Hill sur la déformation plastique. Ceci revient à dire que les forces $A^{k}$ associées aux flux $\dot{a}^{k}$ appartiennent au sous-gradient du potentiel de dissipation $\omega$, soit :

$$
A^{k}=\frac{\partial \omega}{\partial \dot{a}^{k}} .
$$

Réciproquement, à l'aide de la transformation de Legendre-Fenchel, on peut définir le pseudo-potentiel de dissipation $\Omega$ tel que :

$$
\dot{a}^{k}=\frac{\partial \Omega}{\partial A^{k}} .
$$

L'équation (6) représente le cadre général de la viscoplasticité généralisée et l'on voit que la dissipation intrinsèque est positive si $\Omega$ est convexe, positif et qu'il s'annule à l'origine.

Le cas de la plasticité dérive de la viscoplasticité avec l'introduction d'un point singulier (limite élastique). En effet, la déformation plastique étant par définition indépendante du temps, on peut assimiler (du moins dans le cas des théories associées), le pseudo-potentiel $\Omega$ à la fonction indicatrice du convexe $f$ qui représente la surface seuil d'élasticité, soit :

$$
\left.\begin{array}{rlrl}
\Omega\left(A^{k}\right) & =0 & & \text { si } f\left(A^{k}\right)<0 \\
\Omega\left(A^{k}\right) & =+\infty & & \text { si } f\left(A^{k}\right)>0 \\
\dot{a}^{k} & =\dot{\lambda} \frac{\partial f}{\partial A^{k}} & & \text { si } f=0 \text { et } \lambda \geqq 0
\end{array}\right\}
$$

$\dot{\lambda}$ étant le multiplicateur plastique déterminé par la condition de consistance, $f=\dot{f}=0$, ce qui impose au point de chargement de rester sur $f$. Dans la suite de l'article, nous montrerons que chacun des modèles viscoplastiques présentés possède une version asymptotique purement plastique.

A ce stade, il est possible de définir un matériau élastoviscoplastique à plasticité instantanée pour lequel il existe une famille de paramètres internes 
$\alpha^{k}$ telle que si $A^{k}$ désigne l'action thermodynamique associée $\left(\mathcal{A}^{k}=-\frac{\partial \psi}{\partial \alpha^{k}}\right)$, on vérifie l'hypothèse de normalité généralisée conduisant à la vérification $a$ priori de la dissipation positive (Eq. (4)) ; il s'agit de la classe des matériaux standards généralisés. Si l'on fait l'hypothèse de la partition des déformations (Eq. (1)) et que l'on écrit l'énergie libre sous la forme $\psi=\psi\left(\varepsilon_{i j}^{\mathrm{el}}, \alpha_{i j}^{k}, T\right)$, de l'inégalité de ClausiusDuhem (Eq. (2)), on déduit que :

$$
\sigma_{i j}=\rho \frac{\partial \psi}{\partial \varepsilon_{i j}^{\mathrm{el}}}, S=-\frac{\partial \psi}{\partial T} \text { et } \mathcal{A}_{i j}^{k}=\rho \frac{\partial \psi}{\partial \alpha_{i j}^{k}},
$$

et de l'hypothèse de dissipativité normale, on arrive à :

$$
\begin{aligned}
\dot{\varepsilon}_{i j}= & \frac{\partial \Omega\left(\sigma_{k l}, \mathcal{A}^{k}\left(\alpha^{k}\right), T\right)}{\partial \sigma_{i j}}+ \\
& +\dot{\lambda} \frac{\partial f\left(\sigma_{k l}, \mathcal{A}^{k}\left(\alpha_{k}\right), T\right)}{\partial \sigma_{i j}} \\
-\dot{\alpha}_{i j}^{k}= & \frac{\partial \Omega(\ldots)}{\partial \mathcal{A}_{i j}^{k}}+\dot{\lambda} \frac{\partial f(\ldots)}{\partial \mathcal{A}_{i j}^{k}}
\end{aligned}
$$

avec $\lambda \geqq 0$ si $f=0$, plus la condition de chargement plastique $\frac{\partial f}{\partial \sigma_{i j}} \dot{\sigma}_{i j} \geqq 0$ (en l'absence d'adoucissement), $\lambda=0$ si $f \leqq 0$.

Par la suite, nous confrontons les hypothèses de départ de chacun des modèles étudiés à la formulation (9) vérifiant a priori la dissipativité normale. En effet, tout modèle doit être thermodynamiquement correct, c'est-à-dire ne pas mettre en défaut les deux principes de la thermodynamique (Eq. (2)).

\subsection{PARTICULARISATION CLASSIQUE AU CHOIX DES VARIABLES INTERNES.}

4.2.1 Choix des potentiels $\Omega$, f et $\psi$. - Il reste à définir le nombre et la nature des variables internes dont dépendent les potentiels $\Omega$ (et/ou $f$ ) et $\psi$. Le potentiel plastique $f$ est associé à la surface seuil d'élasticité définie dans l'espace des contraintes et son étude expérimentale montre que pour un matériau initialement isotrope, $f$ répond le plus souvent au critère de Von-Misès, soit :

$$
f=J_{2}(\underline{\boldsymbol{\sigma}})-R_{0}^{\mathrm{p}}=0 .
$$

$J_{2}(\underline{\boldsymbol{\sigma}})$ est le deuxième invariant du déviateur $\underline{\mathbf{\sigma}}^{\prime}$ du tenseur des contraintes $\underline{\mathbf{\sigma}}$. Avec la déformation du matériau, cette surface se dilate, se translate et se distord [22-25], les deux premiers effets étant prépondérants.

Pour une approche au premier ordre des phénomènes, ce qui est la situation des modèles envisagés, on néglige la distorsion de cette surface et l'on attribue une variable intensive à chacune des deux autres caractéristiques, à savoir : une (ou plusieurs) variable scalaire d'écrouissage isotrope $R^{\mathrm{p}}$ que l'on associe à sa dilatation et une (ou plusieurs) variable tensorielle d'écrouissage cinématique $X^{\mathrm{p}}$ associée à sa translation. Dans l'ensemble des modèles présentés, on retrouvera constamment ces deux types de variable.

Compte tenu de ces deux effets, on peut réécrire, pour un isotherme, la fonction seuil d'élasticité $f$ sous la forme :

$$
\begin{aligned}
f & =f\left(J_{2}\left(\underline{\mathbf{\sigma}}-\underline{\mathbf{X}}^{\mathrm{p}}\right), R^{\mathrm{p}}, R_{0}^{\mathrm{p}}\right) \\
& =J_{2}\left(\underline{\mathbf{\sigma}}-\underline{\mathbf{X}}^{\mathrm{p}}\right)-R^{\mathrm{p}}-R_{0}^{\mathrm{p}}=0,
\end{aligned}
$$

$J_{2}\left(\underline{\mathbf{\sigma}}-\underline{\mathbf{X}}^{\mathrm{p}}\right)$ est le second invariant du déviateur $\left(\underline{\boldsymbol{\sigma}}^{\prime}-\underline{\mathbf{X}}^{\mathrm{p}^{\prime}}\right)$ du tenseur $\left(\underline{\mathbf{\sigma}}-\underline{\mathbf{X}}^{\mathrm{p}}\right)$.

En ce qui concerne le potentiel $\Omega$, sa détermination est beaucoup plus délicate $[26,27]$ et pose encore de nombreux problèmes expérimentaux. Les quelques résultats disponibles montrent qu'avec la déformation, la famille d'équipotentielles (une équipotentielle est définie à $\dot{\varepsilon}_{i j}^{\mathrm{v}}=$ Cste) a sensiblement les mêmes propriétés que la surface de charge, c'està-dire une forte translation et, suivant les auteurs (ce point reste à préciser) une diminution sensible du numéro d'ordre de ces équipotentielles pour une distance, d'un point de l'espace des contraintes au barycentre de la translation, fixée, et enfin une assez faible distorsion. Ainsi, on écrira d'une manière générale :

$$
\Omega=\Omega_{\mathrm{v}}\left(J_{2}\left(\underline{\mathbf{\sigma}}-\underline{\mathbf{X}}^{\mathrm{v}}\right), R^{\mathrm{v}}, T\right) .
$$

Des phénomènes de restauration peuvent se produire, essentiellement à température élevée lorsque l'agitation thermique est efficace, et ceci même en l'absence de contrainte appliquée. En suivant l'approche de Ponter et Leckie [28], on ajoutera donc à l'équation (12) le potentiel de restauration $\Omega_{\mathrm{r}}$, convexe positif, soit :

$\Omega=\Omega_{\mathrm{v}}\left(J_{2}\left(\underline{\mathbf{g}}-\underline{\mathbf{X}}^{\mathrm{v}}\right), R^{\mathrm{v}}, T\right)+\Omega_{\mathrm{r}}\left(J_{2}\left(\underline{\mathbf{X}}^{\mathrm{v}}\right), R^{\mathrm{v}}, T\right)$,

$J_{2}\left(\underline{X}^{v}\right)$ est le deuxième invariant du déviateur $\underline{\mathbf{X}}^{\mathbf{v}^{\prime}}$ du tenseur cinématique $\underline{\mathbf{X}}^{\mathbf{v}}$.

Bien que ce ne soit pas le but du présent article, mais pour des raisons d'homogénéité et de continuité $\mathrm{du}$ raisonnement, on peut noter que l'endommagement du matériau est traduit dans les modèles i), ii) et iii) par l'introduction d'une variable interne QD (scalaire ou tensorielle) qui généralise le concept de la contrainte effective $\tilde{\tilde{\sigma}}=\underline{\boldsymbol{\sigma}}(\underline{\mathbf{I}}-\mathbb{D})^{-1}$ de Rabotnov-Kachanov [1-3] [29] (I est un tenseur unité).

Ainsi, pour un matériau élasto-viscoplastique endommageable, les variables thermodynamiques envisagées peuvent se classer de la manière suivante : 


\begin{tabular}{|c|c|c|}
\hline \multicolumn{2}{|c|}{$\begin{array}{c}\text { Variables d'états } \\
\text { (paramètres extensifs) } \\
\alpha^{k}\end{array}$} & $\begin{array}{c}\text { Variables associées } \\
\text { (paramètres intensifs) } \\
\mathcal{A}^{k}\end{array}$ \\
\hline observables & internes & \\
\hline$\varepsilon_{i j}^{\mathrm{cl}}$ & & $\sigma_{i j}$ \\
$T$ & & $S$ \\
& $\varepsilon_{i j}^{\mathrm{v}}, \varepsilon_{i j}^{\mathrm{p}}$ & $\sigma_{i j}$ \\
& $\alpha_{i j}^{\mathrm{v}, \mathrm{p}}$ & $X_{i j}^{\mathrm{v}} \mathrm{p}$ \\
& $r^{\mathrm{p}}=p, r^{\mathrm{v}}$ & $R^{\mathrm{p}}, R^{\mathrm{v}}$ \\
& $\mathbb{D}$ & $Y$ \\
\hline
\end{tabular}

Dans ce tableau, $\varepsilon_{i j}, \varepsilon_{i j}^{\mathrm{v}}, \varepsilon_{i j}^{\mathrm{p}}, \varepsilon_{i j}^{\mathrm{el}}, \sigma_{i j}, S$ et $T$ ont la signification donnée précédemment, $r^{\mathrm{p}}$ est la contrainte interne isotrope égale à $p=\int_{0}^{t}$ $\sqrt{\frac{2}{3} \dot{\varepsilon}_{i j}^{\mathrm{p}} \dot{\varepsilon}_{i j}^{\mathrm{p}}} \mathrm{d} t$ dans le cas de la plasticité, $R^{\mathrm{p}}$ le rayon de la surface de plasticité, $\alpha_{\mathrm{ij}}^{\mathrm{p}}$ le tenseur des contraintes internes cinématiques, $X_{i j}^{\mathrm{p}}$ le tenseur de la translation de cette surface, $\mathbb{D}$ la variable interne d'endommagement et $Y$ est le taux de restitution d'énergie d'endommagement. Il en est de même pour les variables visqueuses indicées $\left({ }^{v}\right)$. On adoptera cette notation pour la suite de l'article.

A cette étape, il est possible de définir les variables intensives $X_{i j}^{\mathrm{v}, \mathrm{p}}, R^{\mathrm{v}, \mathrm{p}}, \ldots$, à partir de l'énergie libre $\psi$, soit d'après l'équation (8) :

$$
\begin{gathered}
\sigma_{i j}=\rho \frac{\partial \psi}{\partial \varepsilon_{i j}^{\mathrm{cl}}}, S=-\frac{\partial \psi}{\partial T}, X_{i j}^{\mathrm{v}, \mathrm{p}}=\rho \frac{\partial \psi}{\partial \alpha_{\mathrm{ij}}^{\mathrm{v}, \mathrm{p}}}, \\
R^{\mathrm{v}, \mathrm{p}}=\rho \frac{\partial \psi}{\partial r^{\mathrm{v}, \mathrm{p}}} \quad \text { et } \quad Y=\rho \frac{\partial \psi}{\partial \mathbb{D}} .
\end{gathered}
$$

Si l'on prend l'énergie libre sous une forme quadratique des variables $\alpha_{i j}^{\mathrm{v}, \mathrm{p}}$ (par exemple $\left.J_{2}^{2}\left(\underline{\mathbf{\alpha}}^{\mathbf{v}, \mathbf{p}}\right)\right)$ et en tenant compte du découplage entre élasticité et viscoplasticité, $\psi$ s'écrit :

$\rho \psi=\frac{1}{2} C_{i j k l} \varepsilon_{i j} \varepsilon_{k l}+\frac{2}{9} A^{\mathrm{v}, \mathrm{p}} J_{2}^{2}\left(\underline{\boldsymbol{\alpha}}^{\mathrm{v}, \mathrm{p}}\right)+W\left(r^{\mathrm{v}, \mathrm{p}}\right)$

qui avec (14), donne :

et

$$
\left.\begin{array}{rl}
\sigma_{i j} & =C_{i j k l} \varepsilon_{k l}, X_{i j}^{\mathrm{v}, \mathrm{p}}=\frac{2}{3} A^{\mathrm{v}, \mathrm{p}} \alpha_{i j}^{\mathrm{v}, \mathrm{p}} \\
R^{\mathrm{v}, \mathrm{p}} & =\frac{2}{9}\left(A^{\mathrm{v}, \mathrm{p}}\right)^{\prime} J_{2}^{2}\left(\underline{\alpha}^{\mathrm{v}, \mathrm{p}}\right)+W^{\prime}\left(r^{\mathrm{v}, \mathrm{p}}\right)
\end{array}\right\} .
$$

$A^{\mathrm{v}, \mathrm{p}}$ sont des fonctions qui peuvent respectivement dépendre des paramètres extensifs $r^{\vee}$ ou $p$. Le choix des potentiels $\psi, \Omega$ et $f$ détermine complètement le modèle standard généralisé (Eq. (9)). (On peut remarquer que si au temps $t=0$, on a $\underline{\mathbf{X}}=0$, alors $\underline{\mathbf{X}}$ est un déviateur, ce qui conduit à $X_{i j}=X_{i j}^{\prime}$ ).

4.2.2 Application auX modèles proposés. Pour une meilleure compréhension de cet alinéa, on peut se rapporter aux schémas de principe illustrant les différentes approches des modèles exposés au paragraphe 5 (Fig. 13 à 18) $[7,9,16-17,20,30-37]$.

Les équations d'état définissant la déformation totale sont toujours issues de $f$ et/ou $\Omega$ ou de combinaisons linéaires des équations (11), (12) et (13) ; c'est-à-dire que tous les modèles postulent la première hypothèse de normalité (Eq. (9a)). Dans l'approche unifiée, il n'existe qu'un seul potentiel viscoplastique $\Omega$ correspondant à l'équation (13). Cependant, alors que le modèle ONERA admet l'existence d'une surface seuil $g$ identique à $f$ (Eq. (11)) dont les équipotentielles sont homothétiques et à l'intérieur de laquelle il n'existe que des déformations élastiques, le modèle LMAB néglige la taille de cette surface.

Dans le premier cas, on a :

$$
\dot{\varepsilon}_{i j}^{\mathrm{v}}=\frac{\partial \Omega}{\partial \sigma_{i j}}=\frac{3}{2} H(g) \frac{\partial \Omega_{\mathrm{v}}}{\partial(g)} \frac{\sigma_{i j}^{\prime}-X_{i j}^{\mathrm{v}^{\prime}}}{J_{2}\left(\underline{\mathbf{\sigma}}-\underline{\mathbf{X}}^{\mathrm{v}}\right)},
$$

$H(g)$ est la fonction d'Heaviside, et pour le modèle LMAB,

$$
\dot{\varepsilon}_{i j}^{\mathrm{v}}=\frac{\partial \Omega}{\partial \sigma_{i j}}=\frac{3}{2} \frac{\partial \Omega_{\mathrm{v}}}{\partial J_{2}} \frac{\sigma_{i j}^{\prime}-X_{i j}^{\mathrm{v}^{\prime}}}{J_{2}\left(\underline{\mathbf{\sigma}}-\underline{\mathbf{X}}^{\mathrm{v}}\right)} .
$$

L'approche partitionnée, développée à l'E.M.P., rejoint la formulation de la première équation $(9 a)$ et si l'on admet (contrairement au modèle ONERA, $f \neq g$ ) qu'il existe une fonction seuil $g$ de centre $\underline{\mathbf{X}}^{\mathbf{v}}$ dont les équipotentielles sont homothétiques et une surface seuil plastique $f$ de centre $\underline{\mathbf{X}}^{\mathrm{p}}$, on peut écrire :

$$
\begin{aligned}
\dot{\varepsilon}_{i j}=\frac{3}{2}\left\{H(g) \frac{\partial \Omega_{\mathrm{v}}}{\partial(g)} \frac{\sigma_{i j}^{\prime}-X_{i j}^{\mathrm{v}^{\prime}}}{J_{2}\left(\underline{\mathbf{\sigma}}-\underline{\mathbf{X}}^{\mathrm{v}}\right)}+\right. \\
\left.+\dot{\lambda} H(f) \frac{\sigma_{i j}^{\prime}-X_{i j}^{\mathrm{p}^{\prime}}}{J_{2}\left(\underline{\mathbf{\sigma}}-\underline{\mathbf{X}}^{\mathrm{p}}\right)}\right\}=\dot{\varepsilon}_{i j}^{\mathrm{v}}+\dot{\varepsilon}_{i j}^{\mathrm{p}},
\end{aligned}
$$

$\dot{\lambda}$ est le multiplicateur plastique déterminé par l'équation de consistance $f=\dot{f}=0$.

Le cadre général des modèles multicouches dérive des travaux de Mandel sur les potentiels plastiques multiples ou leurs extensions au cas de la viscoplasticité. On considère que le volume global du matériau peut être divisé en $(n)$ sous-volumes, ou couches, que l'on peut éventuellement assimiler aux grains du polycristal, et que l'on associe soit en parallèle (modèle étudié au CEA, $\varepsilon^{(n)}=\varepsilon$ ), soit en série (modèle préconisé par le LMX, $\sigma^{(n)}=\sigma$ ). Pour chacune des couches, on retrouve le formalisme 
plastique précédent, soit pour la couche $(n)$ possédant une surface seuil $f^{(n)}$ de centre $\underline{\mathbf{X}}^{\mathrm{p}(n)}$ :

$\dot{\varepsilon}_{i j}^{\mathrm{p}}=\dot{\lambda}^{(n)} \frac{\partial f^{(n)}}{\partial \sigma_{i j}^{(n)}}=\frac{3}{2} \dot{\lambda}^{(n)} H\left(f^{(n)}\right) \frac{\sigma_{i j}^{(n)^{\prime}}-X_{i j}^{\mathrm{p}^{(n)^{\prime}}}}{J_{2}\left(\underline{\boldsymbol{\sigma}}^{(n)}-\underline{\mathbf{X}}^{\mathrm{p}(n)}\right)}$

pour le modèle parallèle, $\lambda^{(n)}$ exprimant que la composante du point de charge $\sigma^{(n)}$ reste sur la frontière élastique $f^{(n)} ; f^{(n)}=\dot{f}^{(n)}=0$.

Pour le modèle série :

$$
\dot{\varepsilon}_{i j}^{\mathrm{p}(n)}=\frac{3}{2} \dot{\lambda}^{(n)} H\left(f^{(n)}\right) \frac{\sigma_{i j}^{\prime}-X_{i j}^{\mathrm{p}(n)^{\prime}}}{J_{2}\left(\underline{\mathbf{\sigma}}-\underline{\mathbf{X}}^{\mathrm{p}(n)}\right)},
$$

$\lambda^{(n)}$ indique que la contrainte $\underline{\mathbf{\sigma}}$ reste sur la frontière $f^{(n)}$.

L'extension viscoplastique de (17) et (18) par l'intermédiaire d'une famille de potentiel de dissipation $\Omega^{(n)}$ ne pose pas de problème.

En conclusion, la première hypothèse de normalité est commune à tous ces modèles qui diffèrent essentiellement; à ce niveau, dans le choix de la forme des potentiels convexes, $\Omega_{\mathrm{v}}, \Omega_{\mathrm{r}}$ et $f$.

Il reste à examiner la seconde loi de normalité sur les variables internes $(\mathrm{Eq} .(9 \mathrm{~b}))$, soit d'après l'équation (11) pour un écoulement plastique pur :

$$
\begin{gathered}
\dot{\alpha}_{i j}^{\mathrm{p}}=-\dot{\lambda} \frac{\partial f}{\partial X_{i j}^{\mathrm{p}}}=\frac{3}{2} \dot{\lambda} \frac{\sigma_{i j}^{\prime}-X_{i j}^{\mathrm{p}^{\prime}}}{J_{2}\left(\underline{\mathbf{\sigma}}-\underline{\mathbf{X}}^{\mathrm{p}}\right)}=\dot{\varepsilon}_{i j}^{\mathrm{p}}, \\
\dot{r}^{\mathrm{p}}=-\dot{\lambda} \frac{\partial f}{\partial R^{\mathrm{p}}}=\dot{\lambda}=\sqrt{\frac{2}{3} \dot{\varepsilon}_{i j}^{\mathrm{p}} \dot{\varepsilon}_{i j}^{\mathrm{p}}}=\dot{p},
\end{gathered}
$$

et d'une manière analogue, d'après l'équation (13), pour un écoulement viscoplastique :

$$
\begin{gathered}
\dot{\alpha}_{i j}^{\mathrm{v}}=-\frac{\partial \Omega}{\partial X_{i j}^{\mathrm{v}}}=\dot{\varepsilon}_{i j}^{\mathrm{v}}-\frac{3}{2} \frac{\partial \Omega_{\mathrm{r}}}{\partial J_{2}\left(\underline{X}^{\mathrm{v}}\right)} \frac{X_{i j}^{\mathrm{v}}}{J_{2}\left(\underline{\mathbf{X}}^{\mathrm{v}}\right)}, \\
\dot{r}^{\mathrm{v}}=-\frac{\partial \Omega}{\partial R^{\mathrm{v}}}=\sqrt{\frac{2}{3} \dot{\varepsilon}_{i j}^{\mathrm{v}} \dot{\varepsilon}_{i j}^{\mathrm{v}}}-\frac{\partial \Omega_{\mathrm{r}}}{\partial R^{\mathrm{v}}}=\dot{v}-\frac{\partial \Omega_{\mathrm{r}}}{\partial R^{\mathrm{v}}} .
\end{gathered}
$$

On remarque donc que $\alpha_{i j}^{\mathrm{p}}$ dépend de la déformation courante $\varepsilon_{i j}^{\mathrm{p}}$ et que $r^{\mathrm{p}}$ est identique à la déformation plastique cumulée. Il en est de même pour les variables visqueuses. Dès lors, avec (16), (22) ou (24) et (23) ou (25) on peut définir les variables intensives $X_{i j}^{\mathrm{v}, \mathrm{p}}$ et $R^{\mathrm{v}, \mathrm{p}}$, soit pour la viscoplasticité par exemple :

$$
\begin{aligned}
\dot{X}_{i j}^{\mathrm{v}} & =\frac{2}{3} A^{\mathrm{v}} \dot{\varepsilon}_{i j}^{\mathrm{v}}-A^{\mathrm{v}} \frac{\partial \Omega_{\mathrm{r}}}{\partial J_{2}\left(\underline{\mathbf{X}}^{\mathrm{v}}\right)} \cdot \frac{X_{i j}^{\mathrm{v}}}{J_{2}\left(\underline{\mathbf{X}}^{\mathrm{v}}\right)}, \\
\dot{R}^{\mathrm{v}} & =\frac{1}{3}\left(A^{\mathrm{v}}\right)^{\prime}\left(\dot{\varepsilon}_{i j}^{\mathrm{v}}-\frac{3}{2} \frac{\partial \Omega_{\mathrm{r}}}{\partial J_{2}\left(\underline{\mathbf{X}}^{\mathrm{v}}\right)} \frac{X_{i j}^{\mathrm{v}}}{J_{2}\left(\underline{\mathbf{X}}^{\mathrm{v}}\right)}\right) \times \\
& \times\left(\dot{\varepsilon}_{i j}^{\mathrm{v}}-\frac{3}{2} \frac{\partial \Omega_{\mathrm{r}}}{\partial J_{2}\left(\underline{\mathbf{X}}^{\mathrm{v}}\right)} \frac{X_{i j}^{\mathrm{v}}}{J_{2}\left(\underline{\mathbf{X}}^{\mathrm{v}}\right)}\right)+\dot{W}^{\prime}\left(r^{\mathrm{v}}\right) .
\end{aligned}
$$

Les équations (25), (26) et (27), définissant l'évolution générale des variables internes, vérifient a priori l'hypothèse de dissipativité normale.

A ce point, il est très aisé de voir que les modèles multicouches s'intègrent directement dans ce formalisme et procèdent à l'extension des relations (26) et (27) à chacune des couches. En effet, si $A^{\mathrm{v}}=$ $a^{\mathrm{v}(n)}$ sont des constantes, pour chaque couche, $\varepsilon_{i j}^{\mathrm{p}(n)}=\varepsilon_{i j}$ et $\dot{W}=0$, on peut écrire :

$$
\begin{aligned}
\dot{X}_{i j}^{\mathrm{v}(n)}= & \frac{2}{3} a^{\mathrm{v}(n)} \dot{\varepsilon}_{i j}-a^{\mathrm{v}(n)} \frac{\partial \Omega_{\mathrm{r}}^{(n)}}{\partial J_{2}\left(\underline{\mathbf{X}}^{\mathrm{v}(n)}\right)} \times \\
& \times \frac{X_{i j}^{\mathrm{v}(n)}}{J_{2}\left(\underline{\mathbf{X}}^{\mathrm{v}(n)}\right)} \\
R^{\mathrm{v}(n)}= & R_{0}^{\mathrm{v}(n)}
\end{aligned}
$$

ce qui, avec l'équation (20) et en posant $\underline{\mathbf{X}}^{\mathrm{v}} \equiv \underline{\mathbf{X}}^{\mathrm{p}}$, définit l'approche iv) du CEA. Bien que ces équations vérifient la dissipativité normale, on peut voir dès à présent que ce modèle est hybride puisque pour obtenir les équations (20) et (28) en appliquant les relations (9) des matériaux standards généralisés, il faut supposer que $\Omega^{(n)}$ est limité au potentiel de restauration $\Omega_{\mathrm{r}}^{(n)}$, soit d'après l'équation (13); $\Omega^{(n)}=\Omega_{\mathrm{r}}^{(n)}\left(J_{2}\left(\underline{\mathrm{X}}^{\mathrm{p}}\right), T\right) \cdot \Omega_{\mathrm{v}}^{(n)}$ est nul. Ainsi, l'écoulement ne dérive que de $f^{(n)}$ et les variables internes $\underline{\mathbf{X}}^{\mathrm{p}(n)}$ de $f^{(n)}$ et $\Omega^{(n)}$. Le paramètre temps n'intervient que par la présence de $\Omega_{\mathrm{r}}^{(n)}$. Comme nous le verrons ultérieurement, il serait préférable et aisé d'avoir une version purement viscoplastique, c'est-à-dire que pour chaque couche, l'ensemble des variables $\dot{\varepsilon}_{i j}^{(n)}, X_{i j}^{(n)}, R^{(n)}$ dériveraient d'un potentiel $\Omega_{\mathrm{v}}^{(n)}$ complet.

Pour ce qui concerne le multicouche série purement plastique développé au LMX, son écriture thermodynamique réside dans les équations (21) pour les lois de déformation et (29) pour les lois d'évolution des variables internes:

$$
\begin{aligned}
{\left[\begin{array}{c}
\dot{X}_{i j}^{\mathrm{p}(1)} \\
\vdots \\
\dot{X}_{i j}^{\mathrm{p}(n)}
\end{array}\right] } & =\left[\begin{array}{ccc}
a_{11}^{\mathrm{p}} & \ldots & a_{1 n}^{\mathrm{p}} \\
\vdots & & \vdots \\
a_{n 1}^{\mathrm{p}} & \ldots & a_{n n}^{\mathrm{p}}
\end{array}\right]\left[\begin{array}{c}
\dot{\varepsilon}_{i j}^{\mathrm{p}(1)} \\
\vdots \\
\dot{\varepsilon}_{i j}^{\mathrm{p}(n)}
\end{array}\right] \\
R^{\mathrm{p}(n)} & =R_{0}^{\mathrm{p}(n)}
\end{aligned}
$$

Ainsi, pour $\dot{X}_{i j}^{p(n)}$, on trouve une combinaison linéaire de la version plastique de l'équation (26) $\left(\dot{X}_{i j}^{\mathrm{p}}=\frac{2}{3} A^{\mathrm{p}}(p) \dot{\varepsilon}_{i j}^{\mathrm{p}}\right.$ avec $\left.A^{\mathrm{p}}(p)=a^{\mathrm{p}}\right)$, ce qui s'intègre parfaitement dans le cadre du modèle standard généralisé plastique multiple (Eq. (9) avec $\Omega=$ Cte, pour chacune des couches), d'où la dissipativité positive. Une version purement viscoplastique est également facilement envisageable.

4.3 LiMITATION DE L'ÉCROUISSAGE CINÉMATIQUE LINÉAIRE. - Cependant, pour les modèles multicou- 
ches, la précision de l'aptitude à reproduire les phénomènes expérimentaux est directement liée au nombre de couches, ce qui montre que leur simplicité n'est qu'apparente. En effet, aux températures intermédiaires $\left(T / T_{\mathrm{F}} \simeq 0,3, T_{\mathrm{F}}\right.$ étant le point de fusion) et pour des vitesses de déformation imposées usuelles $\left(10^{-4} \leqq \dot{\varepsilon}^{\mathrm{T}} \leqq 10^{-3} \mathrm{~S}^{-1}\right)$, c'est-à-dire lorsque les effets du temps sont négligeables, la non-linéarité des courbes effort-déformation ne peut être correctement reproduite que lorsque $(n)$ est grand (on peut estimer : $10 \leqq(n) \leqq 20)$. Ce fait provient de la linéarité des termes d'écrouissage cinématique pour chaque couche (écrouissage de Prager $X_{i j}=a \varepsilon_{i j}$ ).

Ainsí, les trois premiers modèles, ne possédant pas le caractère multicouche, ne peuvent se satisfaire d'un écrouissage cinématique linéaire. On peut également s'en persuader en examinant l'effet Bauschinger, où l'on constate une très grande différence entre les modules tangents en traction et en compression pour une même déformation (module compression $\gg$ module en traction). Pour pallier cette déficience, les trois modèles reprennent le concept de mémoire évanescente développé initialement par Armstrong et Frederick [38] et qui écarte la relation biunivoque entre la déformation $\varepsilon_{i j}$ et la variable cinématique $X_{i j}$; on définit un écrouissage cinématique non linéaire à mémoire évanescente fonction du trajet de la déformation.

Pour incorporer ce concept dans le formalisme thermodynamique viscoplastique précédent, il est nécessaire de conserver la variable $\alpha_{i j}^{\mathrm{v}}$ dans le potentiel de dissipation $\Omega$; soit :

$$
\Omega=\Omega_{\mathrm{v}}\left(\underline{\mathbf{\sigma}}, \underline{\mathbf{X}}^{\mathrm{v}}, R^{\mathrm{v}}, T, \underline{\mathbf{\alpha}}^{\mathrm{v}}\right)+\Omega_{\mathrm{r}}\left(\underline{\mathbf{X}}^{\mathrm{v}}, R^{\mathrm{v}}, T\right),
$$

que l'on particularise, en tenant compte de (13), sous la forme :

$$
\begin{aligned}
\Omega=\Omega_{\mathrm{v}}\left(J_{2}\left(\underline{\boldsymbol{\sigma}}-\underline{\mathbf{X}}^{\mathrm{v}}\right), R^{\mathrm{v}},\right. & \left.J_{2}\left(\underline{\mathbf{X}}^{\mathrm{v}}\right), T, J_{2}\left(\underline{\boldsymbol{\alpha}}^{\mathrm{v}}\right)\right)+ \\
& +\Omega_{\mathrm{r}}\left(J_{2}\left(\underline{\mathbf{X}}^{\mathrm{v}}\right), R^{\mathrm{v}}, T\right) .
\end{aligned}
$$

Ainsi, le potentiel de dissipation $\Omega$ dépend tout naturellement des variables thermodynamiques mais également des variables d'état. Comme pour les matériaux standards généralisés classiques, les conditions pour avoir une dissipation positive (Eq. (4)) est que $\Omega$ soit une fonction convexe par rapport à chacune des variables intensives, qu'il soit toujours positif et qu'il contienne l'origine.

Un moyen, certes artificiel, qui conduit à la stricte définition de l'écrouissage cinématique non linéaire est d'écrire (31) sous la forme :

$$
\begin{aligned}
\Omega= & \Omega_{\mathrm{v}}\left(J_{2}\left(\underline{\mathbf{\sigma}}-\underline{\mathbf{X}}^{\mathrm{v}}\right)-R^{\mathrm{v}}-R_{0}^{\mathrm{v}}+\frac{C^{\mathrm{v}}}{2 A^{\mathrm{v}}} J_{2}^{2}\left(\underline{\mathbf{X}}^{\mathrm{v}}\right)-\right. \\
& \left.-\frac{2}{9} C^{\mathrm{v}} A^{\mathrm{v}} J_{2}^{2}\left(\underline{\boldsymbol{\alpha}}^{\mathrm{v}}\right)\right)+\Omega_{\mathrm{r}}\left(J_{2}\left(\underline{\mathbf{X}}^{\mathrm{v}}\right), R^{\mathrm{v}}, T\right) .
\end{aligned}
$$

$C^{\mathrm{v}}$ est une fonction pouvant dépendre de la déformation visqueuse cumulée $v$. En appliquant la normalité généralisée sur les variables internes, (Eq. (9b)), on arrive pour $\dot{\alpha}_{i j}^{\mathrm{v}}$ à :

$$
\begin{aligned}
\dot{\alpha}_{i j}^{\mathrm{v}} & =-\frac{\partial \Omega}{\partial X_{i j}^{\mathrm{v}}} \\
& =\dot{\varepsilon}_{i j}^{\mathrm{v}}-\frac{3}{2} \dot{v} \frac{C^{\mathrm{v}}}{A^{\mathrm{v}}} X_{i j}^{\mathrm{v}}-\frac{3}{2} \frac{\partial \Omega_{\mathrm{r}}}{\partial J_{2}\left(\underline{\mathbf{X}}^{\mathrm{v}}\right)} \cdot \frac{X_{i j}^{\mathrm{v}}}{J_{2}\left(\underline{\mathbf{X}}^{\mathrm{v}}\right)},
\end{aligned}
$$

et en tenant compte de (16), au paramètre $X_{i j}^{\mathrm{v}}$ :

$$
\dot{X}_{i j}^{\mathrm{v}}=\frac{2}{3} A^{\mathrm{v}} \dot{\varepsilon}_{i j}-C^{\mathrm{v}} \dot{v} X_{i j}-A^{\mathrm{v}} \frac{\partial \Omega_{\mathrm{r}}}{\partial J_{2}\left(\underline{\mathbf{X}}^{\mathrm{v}}\right)} \cdot \frac{X_{i j}^{\mathrm{v}}}{J_{2}\left(\underline{\mathbf{X}}^{\mathrm{v}}\right)} \text {. }
$$

Si $A^{\mathrm{v}}(v)=a^{\mathrm{v}}=$ Cste, (34) se simplifie :

$$
\dot{X}_{i j}^{\mathrm{v}}=\frac{2}{3} a^{\mathrm{v}} \dot{\varepsilon}_{i j}^{\mathrm{v}}-C^{\mathrm{v}}(v) \dot{v} X_{i j}^{\mathrm{v}}-a^{\mathrm{v}} \frac{\partial \Omega_{\mathrm{r}}}{\partial J_{2}\left(\underline{\mathbf{X}}^{\mathrm{v}}\right)} \cdot \frac{X_{i j}^{\mathrm{v}}}{J_{2}\left(\underline{\mathbf{X}}^{\mathrm{v}}\right)} .
$$

A l'aide de (35), on voit qu'au terme cinématique linéaire du type Prager, il s'ajoute un terme de rappel, indépendant du temps et fonction du trajet de déformation $\left(\dot{v} X_{i j}\right)$ et un terme de rappel essentiellement fonction du temps. Avec ce type d'écrouissage, on dissocie donc les effets de non-linéarité liés à la déformation et au temps.

On peut remarquer que la présence du terme en $J_{2}^{2}\left(\underline{\boldsymbol{\alpha}}^{\mathrm{v}}\right)$ dans (32) sert uniquement, compte tenu de la relation $X_{i j}^{\mathrm{v}}=\frac{2}{3} A^{\mathrm{v}} \alpha_{i j}^{\mathrm{v}}$, à compenser le terme en $J_{2}^{2}\left(\underline{\mathbf{X}}^{\mathrm{v}}\right)$ servant à décrire l'écrouissage cinématique non linéaire. Ainsi, (32) est strictement analogue à (13), soit :

$$
\begin{aligned}
\Omega=\Omega_{\mathrm{v}}\left(J_{2}\left(\underline{\mathbf{\sigma}}-\underline{\mathbf{X}}^{\mathrm{v}}\right)-R^{\mathrm{v}}-R_{0}^{\mathrm{v}}\right) & + \\
& +\Omega_{\mathrm{r}}\left(J_{2}\left(\underline{\mathbf{X}}^{\mathrm{v}}\right), R^{\mathrm{v}}, T\right),
\end{aligned}
$$

et l'adjonction du terme en $J_{2}^{2}\left(\underline{X}^{v}\right)$ n'affecte pas la limite élastique initiale $R_{0}^{\mathrm{v}}$. Il est assez facile de vérifier la dissipativité positive (Eq. (4)), c'est-àdire : $\sigma_{i j} \dot{\varepsilon}_{i j}^{\mathrm{v}}-X_{i j}^{\mathrm{v}} \quad \dot{\alpha}_{i j}^{\mathrm{v}}-R^{\mathrm{v}} \dot{r}^{\mathrm{v}} \geqq 0$. En explicitant $\dot{\varepsilon}_{i j}^{\mathrm{v}}, \dot{\alpha}_{i j}^{\mathrm{v}}$ et $\dot{r}^{\mathrm{v}}$ par les équations (17), (34), (25), on arrive à l'inéquation :

$$
\begin{aligned}
\left(J_{2}\left(\underline{\mathbf{\sigma}}-\underline{\mathbf{X}}^{\mathrm{v}}\right)-\right. & \left.R^{\mathrm{v}}-R_{0}^{\mathrm{v}}\right) \dot{v}+\left(R_{0}^{\mathrm{v}}+\frac{C(v)}{A(v)} J_{2}^{2}(\underline{\mathrm{X}})\right) \dot{v}+ \\
& +R^{\mathrm{v}} \frac{\partial \Omega_{\mathrm{r}}}{\partial R^{\mathrm{v}}}+J_{2}\left(\underline{\mathbf{X}}^{\mathrm{v}}\right) \cdot \frac{\partial \Omega_{\mathrm{r}}}{\partial J_{2}(\underline{\mathrm{X}})} \geqq 0
\end{aligned}
$$

Le premier terme est positif ou nul, même en l'absence du terme $\left(R^{\mathrm{v}}+R_{0}^{\mathrm{y}}\right)$, cas du modèle LMAB ; les deux derniers l'étant également en assurant la convexité du potentiel de restauration $\Omega_{\mathrm{r}}$. La condition peu restrictive à satisfaire reste donc :

$$
R_{0}^{\mathrm{v}}+\frac{C(v)}{A(v)} J_{2}^{2}\left(\underline{\mathbf{X}}^{\mathrm{v}}\right) \geqq 0,
$$

ce qui est réalisé si $R_{0}^{\mathrm{v}}, C(v)$ et $A(v)$ sont positifs. 
Le modèle de l'ONERA s'inscrit directement dans ce contexte puisque $\dot{X}_{i j}^{v}$ et $\dot{R}^{v}$ sont données par (34) et (27) avec $A(v)=a$.

Comme nous l'avons vu précédemment, celui de l'EMP fait appel, en plus du potentiel $\Omega\left(\sigma_{i j}\right.$, $\left.X_{i j}^{\mathrm{v}}, R^{\mathrm{v}}, T, \alpha_{i j}^{\mathrm{v}}\right)$ déduit de $g$ par homothétie, à une surface seuil de plasticité $f$ qui présente également un écrouissage cinématique non linéaire $X_{i j}^{\mathrm{p}}$ et un écrouissage isotrope $R^{\mathrm{p}}$. Si l'on calque la forme de $f$ sur celle de $\Omega_{\mathrm{v}}$ (Eq. (32)), en laissant de côté les termes de restauration et en posant $A(p)=a^{\mathrm{p}}$, $C(p)=C^{\mathrm{p}}=$ Cstes, on peut écrire les deux potentiels $\Omega$ et $f$ sous la forme :

$$
\left.\begin{array}{rl}
\Omega= & \Omega_{\mathrm{v}}\left(J_{2}\left(\underline{\mathbf{\sigma}}-\underline{\mathbf{X}}^{\mathrm{v}}\right)-R^{\mathrm{v}}-R_{0}^{\mathrm{v}}+\right. \\
& \left.+\frac{C^{\mathrm{v}}}{2 a^{\mathrm{v}}} J_{2}^{2}\left(\underline{\mathbf{X}}^{\mathrm{v}}\right)-\frac{2}{9} C^{\mathrm{v}} a^{\mathrm{v}} J_{2}^{2}\left(\underline{\boldsymbol{\alpha}}^{\mathrm{v}}\right)\right) \\
f= & f_{\mathrm{p}}\left(J_{2}\left(\underline{\mathbf{\sigma}}-\underline{\mathbf{X}}^{\mathrm{p}}\right)-R^{\mathrm{p}}-R_{0}^{\mathrm{p}}+\right. \\
& \left.+\frac{C^{\mathrm{p}}}{2 a^{\mathrm{p}}} J_{2}^{2}\left(\underline{\mathbf{X}}^{\mathrm{p}}\right)-\frac{2}{9} C^{\mathrm{p}} a^{\mathrm{p}} J_{2}^{2}\left(\underline{\boldsymbol{\alpha}}^{\mathrm{p}}\right)\right)
\end{array}\right\}
$$

Cependant, pour tenir compte des interactions plasticité-fluage évoquées précédemment, les auteurs réalisent un couplage au niveau des variables cinématiques $\underline{\mathbf{X}}^{\mathrm{v}}$ et $\underline{\mathbf{X}}^{\mathrm{p}}$. D'un point de vue thermodynamique, ce couplage linéaire peut être réalisé au niveau de l'énergie libre (15), que l'on écrira toujours sous une forme quadratique d'une combinaison linéaire de $\underline{\boldsymbol{\alpha}}^{\mathrm{v}}$ et $\underline{\boldsymbol{\alpha}}^{\mathrm{p}}$, soit :

$$
\begin{array}{r}
\rho \psi=\frac{1}{2} C_{i j k l} \varepsilon_{i j} \varepsilon_{k l}+\frac{2}{9} J_{2}^{2}\left(a^{\mathrm{v}} k^{\vee} \underline{\boldsymbol{\alpha}}^{\mathrm{v}}+a^{\mathrm{p}} k^{\mathrm{p}} \underline{\boldsymbol{\alpha}}^{\mathrm{p}}\right)+ \\
+W\left(r^{\mathrm{p}}\right)+W\left(r^{\mathrm{v}}\right)
\end{array}
$$

En appliquant (14) :

$$
X_{i j}^{\mathrm{v}, \mathrm{p}}=\rho \frac{\partial \psi}{\partial \alpha_{i j}^{\mathrm{v}, \mathrm{p}}} \quad \text { et } \quad R^{\mathrm{v}, \mathrm{p}}=\rho \frac{\partial \psi}{\partial r^{\mathrm{v}, \mathrm{p}}},
$$

la seconde loi de normalité des matériaux standards généralisés (Eq. (9b)), et en posant $\left(a^{\mathrm{v}} k^{\mathrm{v}}\right)^{2}=K^{\mathrm{v}}$, $\left(a^{\mathrm{p}} k^{\mathrm{p}}\right)^{2}=K^{\mathrm{p}}, \quad a^{\mathrm{v}} a^{\mathrm{p}} k^{\mathrm{v}} k^{\mathrm{p}}=K^{\mathrm{vp}}, \quad a^{\mathrm{v}}=a^{\mathrm{p}}=1$, on arrive aux relations de l'EMP, soit:

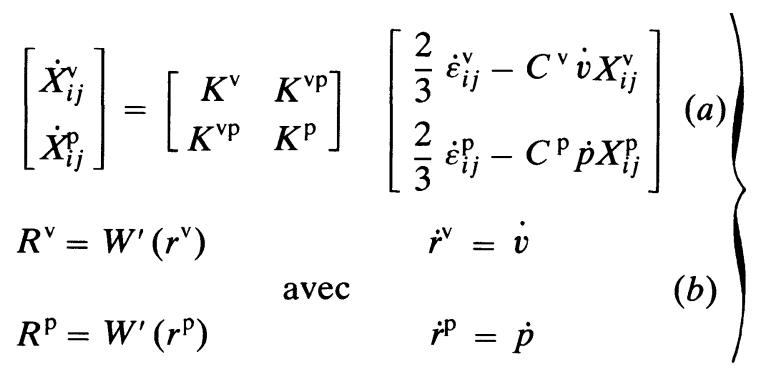

On peut noter que (40) ressemble à l'équation (29) du modèle multicouches du LMX, simplifié à deux couches et avec écrouissages cinématiques non linéaires. Le coefficient $K^{\mathrm{vp}}$ effectue le couplage des variables cinématiques. En suivant une démarche analogue à celle qui nous a conduit à l'inéqua- tion (36), on peut montrer que la dissipativité normale est vérifiée moyennant quelques conditions peu restrictives sur $R_{0}^{\mathrm{v}}, R_{0}^{\mathrm{p}}, C^{\mathrm{v}}, C^{\mathrm{p}}, K^{\mathrm{p}}, K^{\mathrm{v}}$ et $K^{\mathrm{vp}}$, en l'occurrence d'être positifs.

Bien que le modèle LMAB soit assez récent et que son étude thermodynamique complète soit en cours [39], on peut montrer qu'il s'inscrit dans le contexte général développé jusqu'à présent.

On suppose qu'il existe dans l'espace des variables internes une surface $F$, par analogie avec la surface de plasticité classique $f$, qui sépare l'état de charge active $F=0$ de celui des décharges $F<0$. Dans cet espace, on écrira $F$ sous la forme :

$$
F=\frac{2}{3} B J_{2}\left(\underline{\boldsymbol{\alpha}}^{\mathrm{v}}-\underline{\boldsymbol{\alpha}}^{\mathrm{v}(2)}\right)-R^{\mathrm{v}},
$$

$\underline{\boldsymbol{\alpha}}^{\mathrm{v}(2)}$ correspond à la composante cinématique de cette surface (translation) et $R^{\mathrm{v}}$ à sa composante isotrope (gonflement). Lorsque $F=0$, on définit un écoulement macroviscoplastique (indice VM) et à l'opposé, pour $F<0$, un écoulement microviscoplastique (indice $\mathrm{Vm}$ ). Ce modèle, purement cinématique est donc construit à partir de deux variables cinématiques correspondant aux deux états du système, soit $\underline{\boldsymbol{\alpha}}^{\mathrm{vm}}$ et $\underline{\boldsymbol{\alpha}}^{\mathrm{v \textrm {M } ^ { \prime }}}$. On écrira donc l'énergie libre sous la forme :

$$
\begin{aligned}
\rho \psi=\frac{1}{2} C_{i j k l} \varepsilon_{i j} \varepsilon_{k l}+(1 & -H(F)) G^{\mathrm{m}}\left(\underline{\boldsymbol{\alpha}}^{\mathrm{vm}}\right)+ \\
& +H(F) G^{\mathrm{M}}\left(\underline{\boldsymbol{\alpha}}^{\mathrm{vM}^{\prime}}\right) .
\end{aligned}
$$

Comme précédemment, $H(F)$ est la fonction d'Heaviside $(H(F)=1$ si $F=0$ et $H(F)=0$ si $F<0)$. Cependant, $\underline{\boldsymbol{\alpha}}^{\mathrm{vM}^{\prime}}$ peut être considéré comme la somme de plusieurs variables cinématiques, soit $\underline{\boldsymbol{\alpha}}^{\mathrm{vM}}=\sum_{(n)} \underline{\boldsymbol{\alpha}}^{\mathrm{vM}(n)^{\prime}}$ et dans le modèle développé, on prendra $(n)=2$. Moyennant ces hypothèses de départ et en écrivant l'énergie libre sous la forme d'une combinaison linéaire de formes quadratiques des variables, on peut particulariser (42) sous la forme :

$$
\begin{aligned}
\rho \psi=\frac{1}{2} C_{i j k l} \varepsilon_{i j} \varepsilon_{k l}+ & \frac{2}{9} A(1-H(F)) J_{2}^{2}\left(\underline{\boldsymbol{\alpha}}^{\mathrm{vm}}\right)+ \\
& +\frac{2}{9} B H(F) J_{2}^{2}\left(\underline{\boldsymbol{\alpha}}^{\mathrm{vM}}\right) .
\end{aligned}
$$

En appliquant les relations (14) dans les deux états du système, on obtient :

$$
\left.\begin{array}{rl}
X_{i j}^{\mathrm{v}} & =X_{i j}^{\mathrm{vm}}=\frac{2}{3} A \alpha_{i j}^{\mathrm{vm}} \text { pour } H(F)=0 \\
X_{i j}^{\mathrm{v}}=X_{i j}^{\mathrm{vM}} & =\frac{2}{3} B \alpha_{i j}^{\mathrm{vM}} \text { d'où } X_{i j}^{\mathrm{vM}(2)}=\frac{2}{3} B \alpha_{i j}^{\mathrm{vM}(2)} \\
\text { et } \quad X_{i j}^{\mathrm{vM}(1)} & =\frac{2}{3} B \alpha_{i j}^{\mathrm{vM}(1)} \text { pour } H(F)=1
\end{array}\right\} .
$$


Dans ce modèle $\boldsymbol{\alpha}^{\mathrm{vM}(1)}$ n'est pas nul au temps $t=0$, d'où $X_{i j}^{\mathrm{vM}(1)} \neq X_{i j}^{\mathrm{vM}(1)^{\prime}}$ et pour l'homogénéité de l'écriture, on notera l'ensemble des variables sous leur forme déviée même dans les cas $X_{i j}^{\mathrm{vM}}=X_{i j}^{\mathrm{vm}}$ et $X_{i j}^{\mathrm{vM}(2)}=X_{i j}^{\mathrm{vM}(2)^{\prime}}$.

La différenciation entre les deux états s'effectue sur les cinétiques d'évolution de $\underline{\mathbf{\alpha}}^{\mathrm{vm}^{\prime}}$ et $\underline{\boldsymbol{\alpha}}^{\mathrm{vM}^{\prime}}=$ $\alpha^{\mathrm{vM}(1)^{\prime}}+\alpha^{\mathrm{vM}(2)^{\prime}}$ d'où l'existence d'une relation de $\bar{c}^{-}$consistance exprimant que $\underline{\boldsymbol{\alpha}}^{\mathrm{vm}}$ ne peut être supérieure à $\underline{\boldsymbol{\alpha}}^{\mathrm{vM}}$, soit $F=\dot{F}=0$. Cette relation peut s'écrire :

$\frac{2}{3} B^{2}\left(\alpha_{i j}^{\mathrm{vM} \mathrm{M}^{\prime}}-\alpha_{i j}^{\mathrm{vM}(2)^{\prime}}\right)\left(\dot{\alpha}_{i j}^{\mathrm{vM}^{\prime}}-\dot{\alpha}_{i j}^{\mathrm{vM}(2)^{\prime}}\right)=R^{\mathrm{v}} \dot{R}^{\mathrm{v}}$.

Une solution de (44) réside dans (45) :

$$
\alpha_{i j}^{\mathrm{vM}{ }^{\prime}}=\alpha_{i j}^{\mathrm{vM}(2)^{\prime}}+\frac{R^{\mathrm{v}}}{\dot{R}^{\mathrm{v}}}\left(\dot{\alpha}_{i j}^{\mathrm{vM}}-\dot{\alpha}_{i j}^{\mathrm{vM}(2)^{\prime}}\right)
$$

De cette équation, il vient que $\alpha_{i j}^{\mathrm{vM}(1)^{\prime}}=\frac{R^{\mathrm{v}}}{\dot{R}^{\mathrm{v}}}$ $\left(\dot{\alpha}_{i j}^{\mathrm{vM}}-\dot{\alpha}_{i j}^{\mathrm{vM}(2)^{\prime}}\right)$, c'est-à-dire que la variable cinématique $\alpha_{i j}^{\mathrm{vM}(1)^{\prime}}$ dépend du paramètre scalaire $R^{\mathrm{v}}$ et de sa loi d'évolution. Des relations (43), on peut déduire l'écriture de $F$ et des relations de consistance, en fonction des paramètres intensifs $X_{i j}$, soit :

$$
\left.\begin{array}{rl}
F & =J_{2}\left(\underline{\mathbf{X}}^{\mathrm{vM}}-\underline{\mathbf{x}}^{\mathrm{vM}(2)}\right)-R^{\mathrm{v}}=0 \\
\text { et } \quad X_{i j}^{\mathrm{vM} \mathrm{M}^{\prime}} & =X_{i j}^{\mathrm{vM}(2)^{\prime}}+X_{i j}^{\mathrm{vM}(1)^{\prime}} \\
& =X_{i j}^{\mathrm{vM}(2)^{\prime}}+\frac{R^{\mathrm{v}}}{\dot{R}^{\mathrm{v}}}\left(\dot{X}_{i j}^{\mathrm{vM}^{\prime}}-\dot{X}_{i j}^{\mathrm{vM}(2)^{\prime}}\right)
\end{array}\right\},
$$

ce qui constitue une partie des équations du modèle LMAB. Il reste à écrire le pseudo-potentiel de dissipation $\Omega$.

En s'inspirant de l'écriture (32) et en restant le plus général possible quant à la forme des lois d'évolution de $X_{i j}^{\mathrm{vm}}$ et $X_{i j}^{\mathrm{vM}}$ (introduction des fonctions $\mathcal{L}(H(F))$ et $\mathcal{L}^{*}(H(F))$, on peut écrire $\Omega$ sous la forme :

avec

$$
\begin{aligned}
& \Omega=\Omega_{\mathrm{v}}\left(J_{2}\left(\underline{\mathbf{g}}-\underline{\mathbf{X}}^{\mathrm{v}}\right)+\frac{\mathfrak{L}^{*}}{2 \mathfrak{L}} J_{2}^{2}\left(\underline{\mathbf{X}}^{\mathrm{v}}\right)-\frac{2}{9} \mathfrak{L} \mathcal{L} J_{2}^{2}\left(\underline{\boldsymbol{\alpha}}^{\mathrm{v}}\right)\right)+\Omega_{\mathrm{r}}\left(J_{2}\left(\underline{\mathbf{X}}^{\mathrm{v}}\right), T\right) \\
& \left.\mathfrak{L}(H(F))=A(1-H(F))+B H(F) \quad \text { et } \quad \mathfrak{L}^{*}=a^{*}(1-H(F))+b^{*}(H(F))\right\}
\end{aligned}
$$

où $a^{*}$ et $b^{*}$ sont des constantes.

De la première règle de normalité, on retrouve tout naturellement la loi (18), soit :

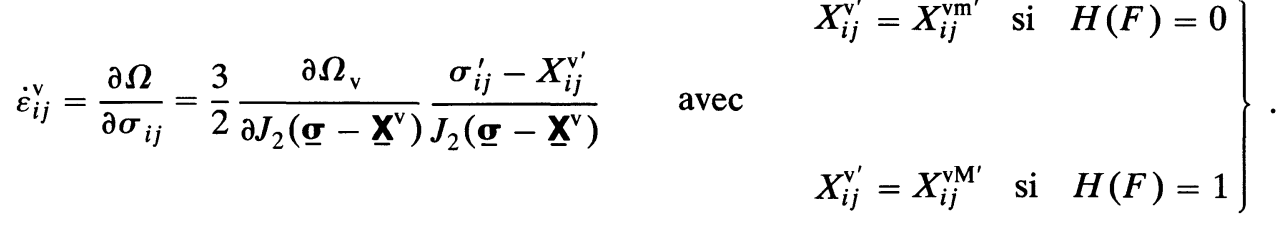

Il n'existe donc qu'une seule loi d'écoulement incluant la plasticité, la viscosité et la microplasticité, la différence s'effectuant sur le choix de $\Omega_{\mathrm{v}}$ et sur les cinétiques de $X_{i j}^{\mathrm{v}}$.

En appliquant la seconde loi de normalité des matériaux standards, on aboutit à :

$$
\dot{\alpha}_{i j}^{\mathrm{v}^{\prime}}=-\frac{\partial \Omega}{\partial X_{i j}^{\mathrm{v}}}=\dot{\varepsilon}_{i j}^{\mathrm{v}}-\frac{3}{2} \frac{\mathcal{L}^{*}}{\mathfrak{L}} \dot{v} X_{i j}^{\mathrm{v}^{\prime}}-\frac{3}{2} \frac{\partial \Omega_{\mathrm{r}}}{\partial J_{2}\left(\underline{\mathbf{X}}^{\mathrm{v}}\right)} \cdot \frac{X_{i j}^{\mathrm{v}^{\prime}}}{J_{2}\left(\underline{\mathbf{X}}^{\mathrm{v}}\right)} .
$$

En explicitant chaque état de $\alpha_{i j}^{\mathrm{v}^{\prime}}$ et à l'aide de (43), on peut définir les lois d'évolution des $X_{i j}^{\mathrm{v}^{\prime}}$, soit :

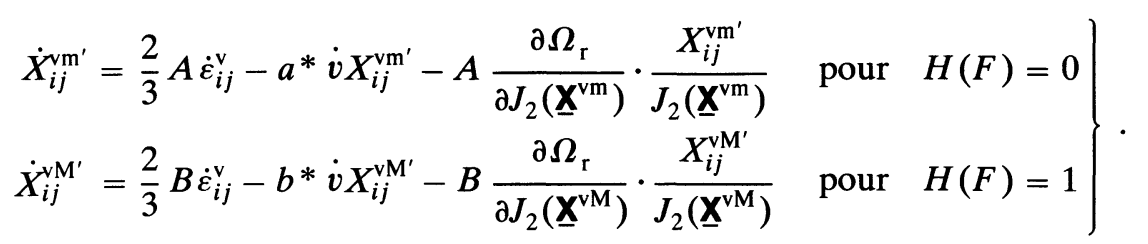

On a donc deux équations cinématiques non linéaires avec restauration que l'on peut éventuellement réduire au cas linéaire en prenant $a^{*}$ et/ou $b^{*}=0$, ce qui est le cas de la microviscoplasticité où $a^{*}=0$.

En tenant compte de (46), on définit donc la forme de l'ensemble des lois d'évolution des variables internes du modèle LMAB ; soit en résumant : 


$$
\begin{aligned}
\dot{X}_{i j}^{\mathrm{vm}} & =\frac{2}{3} A \dot{\varepsilon}_{i j}^{\mathrm{v}}-A \frac{\partial \Omega_{\mathrm{r}}}{\partial J_{2}\left(\underline{\mathbf{X}}^{\mathrm{vm}}\right)} \cdot \frac{X_{i j}^{\mathrm{vM}}}{J_{2}\left(\underline{\mathbf{X}}^{\mathrm{vm}}\right)} \\
\dot{X}_{i j}^{\mathrm{vM}(2)^{\prime}} & =\frac{2}{3} B \dot{\varepsilon}_{i j}^{\mathrm{v}}-b^{*} \dot{v} X_{i j}^{\mathrm{vM}(2)^{\prime}}-B \frac{\partial \Omega_{\mathrm{r}}}{\partial J_{2}\left(\underline{\mathbf{X}}^{\mathrm{vM}}\right)} \cdot \frac{X_{i j}^{\mathrm{vM}(2)^{\prime}}}{J_{2}\left(\underline{\mathbf{X}}^{\mathrm{vM}}\right)} \\
\dot{X}_{i j}^{\mathrm{vM}(1)^{\prime}} & =-b^{*} \dot{v} X_{i j}^{\mathrm{vM}(1)^{\prime}}-B \frac{\partial \Omega_{\mathrm{r}}}{\partial J_{2}\left(\underline{\mathbf{X}}^{\mathrm{vM}}\right)} \cdot \frac{X_{i j}^{\mathrm{vM}(1)^{\prime}}}{J_{2}\left(\underline{\mathbf{X}}^{\mathrm{vM}}\right)} \text { avec } X_{i j}^{\mathrm{vM}(1)^{\prime}}=\frac{R^{\mathrm{v}}}{\dot{R}^{\mathrm{v}}}\left(\dot{X}_{i j}^{\mathrm{vM}}-\dot{X}_{i j}^{\mathrm{vM}(2)^{\prime}}\right)
\end{aligned}
$$

D'après (51c), la loi d'évolution de $R^{\mathrm{v}}$ fixe celle de $X_{i j}^{\mathrm{vM}(1)^{\prime}}$, soit d'après $(46)$ et $(51 \mathrm{c})$ :

$$
\dot{R}^{v}=-b^{*} \dot{v} R^{\mathrm{v}}-B R^{\mathrm{v}} \frac{\partial \Omega_{\mathrm{r}}}{\partial J_{2}\left(\underline{\mathrm{X}}^{\mathrm{vM}}\right)} \cdot \frac{1}{J_{2}\left(\underline{\mathbf{X}}^{\mathrm{vM}}\right)} .
$$

En toute généralité, comme précédemment, $b^{*}$ peut être une fonction de $v$ donc de $R^{v}$ et officie comme la fonction $\dot{W}^{\prime}\left(r^{v}\right)$ des modèles précédents.

On se retrouve dans une situation assez voisine de celles des modèles de l'ONERA et l'EMP, et la vérification de l'inégalité de Clausius-Duhem par l'intermédiaire d'un traitement du type de l'équation (36) ne pose pas de problème. Les conditions requises sont que $\Omega_{\mathrm{r}}$ soit un convexe et que les divers coefficients ou fonctions $A, B, a^{*}, b^{*}$ soient positifs. On peut noter que dans ce modèle $\Omega_{\mathrm{r}}$ intervient dans toutes les cinétiques des variables, ce qui peut être interprété, moyennant la mise en facteur de certains termes d'écrouissage (paragraphe ultérieur), comme l'indifférence de l'écoulement stationnaire à l'état du système, propriété vérifiée expérimentalement (Fig. 5 par exemple).

A ce point du développement thermodynamique, il semble nécessaire d'évoquer d'une manière succincte les travaux récents de Chrysochoos [30] relatifs aux études théorique et expérimentale du bilan énergétique lors d'un essai de traction à vitesse imposée. En effet, en plus de la vérification de la dissipativité positive, les résultats expérimentaux d'un tel bilan suggère un critère complémentaire de validité des modèles. Dans ce contexte énergétique, une étude détaillée et comparative des modèles présentés serait possible et souhaitable, mais trop lourde et en limite du sujet du présent article, par conséquent, nous nous contenterons simplement de résumer les principales conclusions issues des études microcalorimétriques. L'énergie $W_{\text {cxt }}$ nécessaire à la déformation d'un matériau élastoplastique peut être décomposée en une partie élastique $W_{\mathrm{c}}$, réversible, et une partie complémentaire $W_{\mathrm{a}}$ qui se transforme partiellement en chaleur $W_{\mathrm{c}}$, de sorte que : $W_{\mathrm{cxt}}=$ $W_{\mathrm{c}}+W_{\mathrm{c}}+W_{\mathrm{b}}$. Ainsi $W_{\mathrm{b}}$ représente le blocage d'énergie attribuée aux contraintes internes après décharge des efforts extérieurs et correspond à la modification de la microstructure de la matière. La fraction d'énergie bloquée est donnée par le rapport $\mathcal{F}=\frac{W_{\mathrm{b}}}{W_{\mathrm{a}}}=1-\frac{W_{\mathrm{c}}}{W_{\mathrm{a}}} . \quad$ Expérimentalement $W_{\mathrm{c}}$ est accessible par des mesures microcalorimétriques [30] et $W_{\mathrm{a}}$ est directement calculable à partir de la forme de la courbe de traction, d'où l'évaluation de $\mathcal{F}$. Chrysochoos montre que $\mathcal{F}$ décroît avec la déformation (du moins pour l'alliage d'aluminium étudié), ce qui peut constituer un test supplémentaire de validité des modèles thermodynamiques. En effet, d'un point de vue théorique, il montre qu'en grandes déformations, quel que soit le choix de la décomposition élastoplastique des déformations, pour un essai de traction simple, l'écriture du bilan énergétique est unique et se ramène à son écriture en petites déformations, soit d'après les équations (4) et (8) :

$$
D=\dot{W}_{\mathrm{c}}=\sigma \dot{\varepsilon}^{\mathrm{p}}-\rho \frac{\partial \psi}{\partial \alpha^{k}} \dot{\alpha}^{k} .
$$

Dès lors, la confrontation énergétique modèleexpérience est possible et ne pose pas de problème conceptuel. A l'exemple d'un certain nombre de modèles simples [30], le modèle standard généralisé pris dans sa forme primitive (Eqs. (7), (12), (14) et (16) prévoit, contrairement à l'expérience, une croissance continue de $\mathcal{F}$. Ce point de désaccord reste à préciser mais l'une des issues possibles réside dans la structure du type Bailey-Orowan des équations d'évolutions des variables internes $\dot{\alpha}^{k}$, reflétant la compétition entre les termes d'écrouissage et de restauration (dynamique et statique) respectivement associés à la multiplication et à l'annihilation des défauts linéaires. On montre [39] que ce type d'équation conduit à une augmentation rapide puis à une lente diminution de $\mathcal{F}$ avec la déformation, l'ordre de grandeur du maximum calculé ( $40 \leqq$ $\mathcal{F} \leqq 60 \%$ pour $\varepsilon \simeq 1$ à $2 \%$ ) étant compatible avec les mesures calorimétriques directes [30]. Comme nous le verrons ultérieurement, presque tous les modèles décrits possèdent des termes de restauration d'où l'éventuelle possibilité de restituer la décroissance de $\mathcal{F}$.

En conclusion, ce type d'étude, certes très délicate mais très riche et prometteuse, devrait contribuer à l'amélioration de la formulation des modèles à variables cachées. 


\section{Etude des différents modèles.}

5.1 ORGANIGRAMMES DE FONCTIONNEMENT DE CES MODĖLES. - Avant d'exposer les détails de ces modèles, il semble utile de préciser d'une manière concise, les hypothèses de départ des différentes formulations (hypothèses évoquées dans l'approche thermodynamique du paragraphe 4) et de considérer globalement les organigrammes de construction et de fonctionnement de ces approches. Ainsi, à l'aide des schémas de principe des figures 13 à 16 , on visualise aisément dans leurs espaces la position des différentes surfaces plastique et viscoplastique, ce qui constitue la base de l'individualité de chacun des modèles.

\subsubsection{Les modèles phénoménologiques globaux.}

5.1.1.1 Modèle ONERA, LMT Cachan [14, 16, 17, 20]. - Sur la figure 13, dans l'espace du tenseur des contraintes déviées $\left(\sigma_{1}^{\prime}, \sigma_{2}^{\prime}, \sigma_{3}^{\prime}\right)$, à l'instant $t$, il existe une surface limite d'écoulement $f=0$, à l'intérieur de laquelle le comportement est purement élastique. Cette surface a pour rayon $\left(R^{\mathrm{v}}+R_{0}^{\mathrm{v}}\right)$ et pour centre $\underline{\mathbf{X}}^{\mathbf{v}^{\prime}}$. Les surfaces équipotentielles (en pointillés) d'égale dissipation, $\Omega=$ Cte, se déduisent par homothétie par rapport à $f$ (théorie associée).

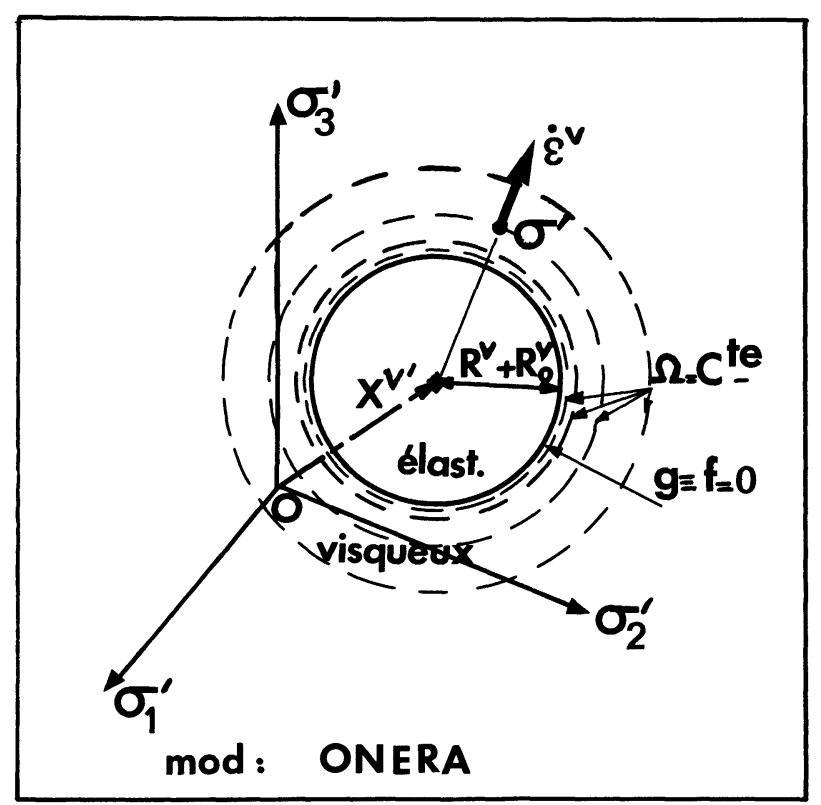

Fig. 13. - Schéma de principe du modèle ONERA-LMT Cachan.

[Basic sketch of the ONERA, LMT model.]

Ainsi, pour $f \geqq 0$, l'écoulement est visqueux et le tenseur des vitesses de déformation, $\dot{\varepsilon}_{i j}^{v}$ unique, s'obtient selon la première hypothèse de normalité.

En résumé, la surface seuil $f$ a pour équation :

$$
f=J_{2}\left(\underline{\mathbf{\sigma}}-\underline{\mathbf{X}}^{\mathrm{v}}\right)-R^{\mathrm{v}}-R_{0}^{\mathrm{v}}=0,
$$

et le potentiel viscoplastique $\Omega$ prend la forme :

$$
\begin{aligned}
\Omega=\Omega_{\mathrm{v}}\left\langle\left( J_{2}\left(\underline{\mathbf{\sigma}}-\underline{\mathbf{X}}^{\mathrm{v}}\right)-\right.\right. & \left.\left.R^{\mathrm{v}}-R_{0}^{\mathrm{v}}\right)\right\rangle+ \\
& +\Omega_{\mathrm{r}}\left(J_{2}\left(\underline{\mathbf{X}}^{\mathrm{v}}\right), R^{\mathrm{v}}\right) .
\end{aligned}
$$

$\langle-\rangle$ signifie que $\langle x\rangle=x$ si $x \geqslant 0$ et $\langle x\rangle=0$ si $x<0$, soit $\langle x\rangle=x H(x)$. Conformément à l'équation (17) traduisant l'hypothèse de normalité (Fig. 13), les vitesses de déformation visqueuses s'écrivent :

$$
\dot{\varepsilon}_{i j}^{\mathrm{v}}=\frac{\partial \Omega(\ldots)}{\partial \sigma_{i j}} .
$$

Les lois d'évolution des variables internes, cinématique $\underline{X}^{\mathrm{v}}$ et isotrope $R^{\mathrm{v}}$, décrivant $f$ et $\Omega$ se déduisent, conformément au paragraphe précédent, de l'écriture de l'énergie libre et de l'application de la seconde loi de normalité. La cinétique de $\underline{X}^{v}$ est du type non linéaire à mémoire évanescente, donnée par l'équation (35), et celle de $R^{\mathrm{v}}$, fonction de $\dot{v}$, est représentée par l'équation (27), soit pour l'ensemble :

$$
\left.\begin{array}{rl}
\dot{X}_{i j}^{\mathrm{v}} & =X\left(\dot{\varepsilon}_{i j}^{\mathrm{v}}, \dot{v}, X_{i j}^{\mathrm{v}}, J_{2}\left(\underline{\mathbf{X}}^{\mathrm{v}}\right), T\right) \\
\dot{R}^{\mathrm{v}} & =R\left(\dot{v}, R^{\mathrm{v}}, T\right)
\end{array}\right\} .
$$

Ce modèle est décrit par les équations (53) et la particularisation, souvent à partir des expériences, des différentes fonctions, sera abordée ultérieurement. Cependant, on peut remarquer que la version plastique, indépendante du temps, de ce modèle, consiste à confondre les surfaces d'égale dissipation avec la surface seuil $f=0$. Dans ce cas, la contrainte ne peut pas sortir de $f$, ce qui est imposé par l'écriture d'une équation de consistance que l'on doit associer à un critère de charge-décharge.

5.1.1.2 Modèle du LMAB [7, 9, 40]. - Contrairement au modèle précédent, celui du LMAB n'admet pas de surface seuil $f=0$ (Fig. 14a), c'est-à-dire que le matériau est toujours visqueux.

Cette hypothèse de travail est fondée sur l'étude, en fluage stationnaire sous sollicitation biaxée de traction-torsion et par la méthode des décréments de contrainte, des surfaces équipotentielles par le biais des directions d'écoulement [27, 40]. Quelques déterminations sur le 17-12 SPH sont proposées sur la figure $14 \mathrm{~b}$. On montre ainsi la forte translation (cinématique) de ces surfaces et l'extrême faiblesse du caractère isotrope puisque le matériau s'écoule dans tout l'espace des contraintes pour un point de chargement fixé ; point (A) de référence pour l'état stationnaire. L'analyse des directions instantanées révèle que l'on est sensiblement normal à des ellipses du type Von-Misès centrées sur les coordonnées $\left(X_{z z}, X_{z \vartheta}\right)$ du vecteur cinématique, correspondant aux points d'inversion du signe de chacune des composantes de la vitesse $\left(\dot{\varepsilon}_{z z}, \dot{\varepsilon}_{z \vartheta} \gtrless 0\right.$, Fig. 14b). 


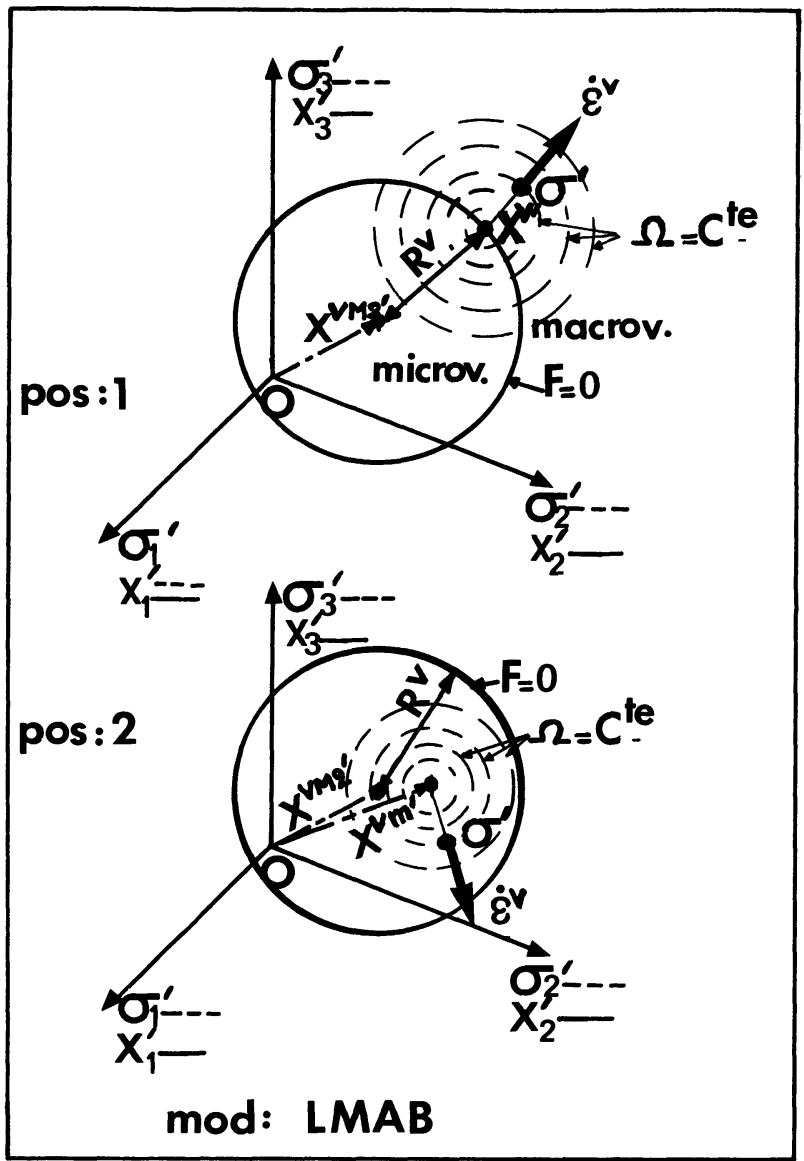

a)

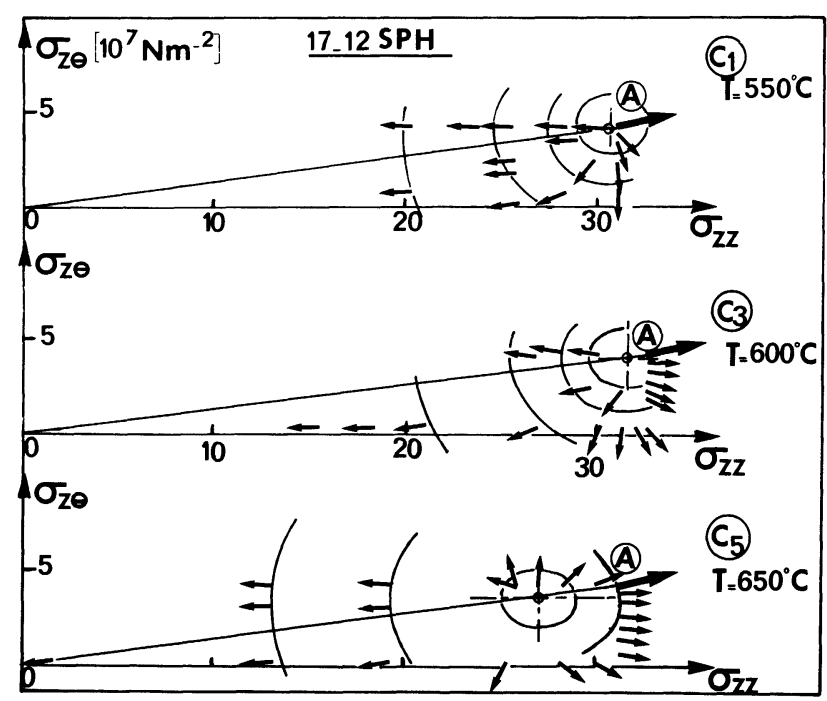

b)

Fig. 14. - a) Schéma de principe du modèle LMAB, b) exemple de détermination expérimentale des surfaces $\Omega=$ Cste, cas de l'inoxydable 17-12 SPH à $T=550,600$ et $650{ }^{\circ} \mathrm{C}$. (D'après Delobelle, Oytana et Mermet [39], avec l'amabilité de ICM IV et de Pergamon Press).

(a) Basic sketch of the LMAB model. b) An example of the experimental determination of the surfaces $\Omega=$ Const., case of $17-12 \mathrm{SPH}$ at $T=550,600$ and $650{ }^{\circ} \mathrm{C}$. (From Delobelle, Oytana and Mermet [39] with courtesy of ICM IV and Pergamon Press).]
Cette observation est parfaitement vérifiée par les directions d'écoulement sous chargement radial qui sont du type Misès. Ainsi, la nature essentiellement visqueuse et cinématique de l'écoulement, du moins pour le 17-12 SPH et quelques autres matériaux monophasés [27], semble démontrée. De plus, en fluage, l'analyse comparée des vitesses stationnaires sous différents niveaux de contrainte $\bar{\sigma}$ à celles des vitesses transitoires instantanées sous $\overline{\sigma-\Delta \sigma}$ montre l'existence d'une courbe maîtresse, $\overline{\dot{\varepsilon}}=$ $\left(\left(\underline{\sigma}-\underline{\mathbf{X}}^{v}\right), T\right)$, d'où l'unicité de la vitesse de déformation $\overline{\dot{\varepsilon}}$ et la nature unifiée du modèle. Cependant, pour introduire la forte dissymétrie entre les déformations correspondant à un premier chargement et celles d'un déchargement, il est nécessaire d'introduire un critère de charge-décharge portant sur $\underline{X}^{\mathrm{V}}$ et associé à une surface $F$, exprimée dans l'espace des contraintes internes déviées $\underline{\mathbf{X}}^{\mathbf{v}^{\prime}}$, permettant d'assurer la continuité de la vitesse de déformation entre les deux états du système.

Conformément aux schémas de la figure 14a, les caractéristiques de ce modèle peuvent se résumer ainsi : il n'existe pas de surface seuil $f$ dans l'espace des contraintes déviées, mais par contre, dans cet espace, le potentiel viscoplastique prend la forme simple :

$$
\Omega=\Omega_{\mathrm{v}}\left(J_{2}\left(\underline{\mathbf{g}}-\underline{\mathbf{X}}^{\mathrm{v}}\right)\right)+\Omega_{\mathrm{r}}\left(J_{2}\left(\underline{\mathbf{X}}^{\mathrm{v}}\right)\right)
$$

(voir Eq. (47)).

La première hypothèse de normalité, vérifiée expérimentalement, conduit à :

$$
\dot{\varepsilon}_{i j}^{v}=\frac{\partial \Omega(\ldots)}{\partial \sigma_{i j}} \quad(\text { voir Eq. (18)) . }
$$

Dans l'espace des contraintes internes cinématiques déviées (traits pleins sur la Fig. 14a), il existe une surface $F$ associée à un critère et permettant de distinguer l'état de charge de celui de décharge. Conformément à l'équation (46), $F$ s'écrit :

$$
F=J_{2}\left(\underline{\mathbf{X}}^{\mathrm{v}}-\underline{\mathbf{X}}^{\mathrm{vM}(2)}\right)-R^{\mathrm{v}}=0,
$$

$X^{\mathrm{vM}(2)}$ et $R^{\mathrm{v}}$ correspondent respectivement à la translation et au grossissement de cette surface. A l'aide de $F$, on définit un état de charge "active » $F=0$ (indice $\mathrm{VM}$ ) conduisant à des déformations que l'on qualifiera de macroviscoplastiques et à l'inverse un état de charge «passive », $F<0$ (indice vm) donnant des déformations microviscoplastiques.

L'état actif résulte de ce que si $F=0$, alors :

$$
\left.\begin{array}{rl}
\dot{X}_{i j}^{\mathrm{vM}(2)^{\prime}} & =X^{\mathrm{vM}}\left(\dot{\varepsilon}_{i j}^{\mathrm{v}}, \dot{v}, X_{i j}^{\mathrm{vM}(2)^{\prime}}, J_{2}\left(\underline{\mathrm{x}}^{\mathrm{vM}}\right), T\right) \\
\dot{R}^{\mathrm{v}} & =R\left(\dot{v}, R^{\mathrm{v}}, T\right)
\end{array}\right\},
$$

et l'équation de consistance $F=\dot{F}=0$ fixant $\underline{\mathbf{X}}^{\mathrm{v}^{\prime}}$ sur la surface $F$, entraîne les relations sur les composantes $X_{i j}^{\mathrm{v}^{\prime}}$, soit d'après $(46)$ : 


$$
X_{i j}^{\mathrm{v}^{\prime}}=X_{i j}^{\mathrm{vM}}=X_{i j}^{\mathrm{vM}(2)^{\prime}}+\frac{R^{\mathrm{v}}}{\dot{R}^{\mathrm{v}}}\left(\dot{X}_{i j}^{\mathrm{vM}}-\dot{X}_{i j}^{\mathrm{vM}(2)^{\prime}}\right) .
$$

Cette situation est transcrite sur la figure 14a, position (I). A l'exemple du modèle précédent, on prendra une cinétique non linéaire à mémoire évanescente pour $\dot{X}_{i j}^{\mathrm{vM}(2)}$ et une fonction scalaire dépendant de $\dot{v}$ pour $\dot{R}^{v}$. On rappelle que dans ce modèle $\dot{R}^{\vee}$ représente l'évolution du module de $\underline{\underline{X}}^{\mathrm{vM}(1)}$.

L'état passif est défini par:

si,

$$
\left.\begin{array}{l}
F<0 \\
F=0 \\
\dot{J}_{2}\left(\underline{\mathbf{X}}^{\mathrm{v}}\right)<0
\end{array}\right\}
$$

alors $\dot{X}_{i j}^{\mathrm{vM}(2)^{\prime}}$ et $\dot{R}^{\vee}$ sont données par (54d), et

$$
X_{i j}^{\mathrm{v}^{\prime}}=X_{i j}^{\mathrm{vm}^{\prime}}=X^{\mathrm{vm}}\left(\dot{\varepsilon}_{i j}, X_{i j}^{\mathrm{vm}^{\prime}}, J_{2}\left(\underline{\mathbf{X}}^{\mathrm{vm}}\right), T\right) .
$$

Cette situation est transposée sur la figure 14a, position (II).

L'organigramme de ce modèle réside donc dans l'ensemble des équations (54). Sa version purement plastique consiste à confondre les surfaces équipotentielles avec le point $\underline{\mathbf{X}}^{\mathbf{v}^{\prime}}$, c'est-à-dire que $\underline{\mathbf{X}}^{\mathbf{v}^{\prime}}=$ $\underline{\mathbf{\sigma}}^{\prime}$. Dans ce cas, la surface $F$ est identique à la surface seuil $f$ et si de plus le coefficient d'écrouissage de $\underline{\mathbf{X}}^{\mathrm{vm}^{\prime}}$ est infini (état microplastique), alors la décharge est purement élastique et l'on se retrouve, comme pour le modèle ONERA, dans la situation élastoplastique classique à écrouissage cinématique et isotrope.

On peut noter que ce modèle supporte complètement le vocable unifié puisque $\dot{\varepsilon}_{i j}^{\mathrm{v}}$ intègre non seulement les déformations plastiques et visqueuses lors d'un chargement (comme pour le modèle ONERA) mais également les faibles déformations de recouvrance lors d'une décharge (partielle ou totale), souvent assimilées, plus ou moins à tort, à de l'anélasticité. En effet, on peut montrer qu'expérimentalement, les déformations de recouvrance vérifient la propriété de normalité (Eq. (48), indice vm) [10], d'où son introduction dans le modèle sous le qualificatif de microviscoplasticité.

On peut noter une certaine analogie avec le modèle de Kujawski, Mroz [41] au niveau de la décomposition de la variable interne cinématique en deux composantes $\underline{\mathbf{X}}^{(1)}, \underline{\mathbf{X}}^{(2)}$, permettant de traduire respectivement la plasticité indépendante et dépendante du temps. En fait, dans leur approche, il s'agit d'une extension du modèle purement plastique de Mroz [42], la déformation étant unique et la surface seuil associée s'exprimant par $f=J_{2}\left(\underline{\mathbf{g}}-\underline{\mathbf{X}}^{(1)}-\right.$ $\left.\underline{\mathbf{X}}^{(2)}\right)-R^{2}(p)=0$. Une autre analogie avec une version viscoplastique du modèle de Mroz [43] est la distinction, par le biais de la mémorisation de la norme de la valeur maximale de la contrainte interne cinématique atteinte $\left(J_{2 \mathrm{~m}}=\operatorname{Sup} J_{2}(\underline{\mathbf{X}})\right)$, entre processus actif et passif durant un chargement quelconque de fluage.

5.1.1.3 Modèle de l'EMP [31, 32]. - On rappelle qu'il s'agit d'un modèle partitionné, c'est-à-dire que la déformation inélastique totale est la somme des déformations plastique et visqueuse, soit $\varepsilon_{i j}^{\mathrm{T}}=\varepsilon_{i j}^{\mathrm{P}}+$ $\varepsilon_{i j}^{v}$ (Fig. 15). En fait, nous verrons par la suite que les approches unifiées et partitionnées représentent deux démarches pour traiter la très forte non-linéarité des caractéristiques expérimentales : $\bar{\varepsilon}=$ $f\left(\sigma_{\mathrm{v}}\right)_{T}, \sigma_{\mathrm{v}}$ étant la composante visqueuse.

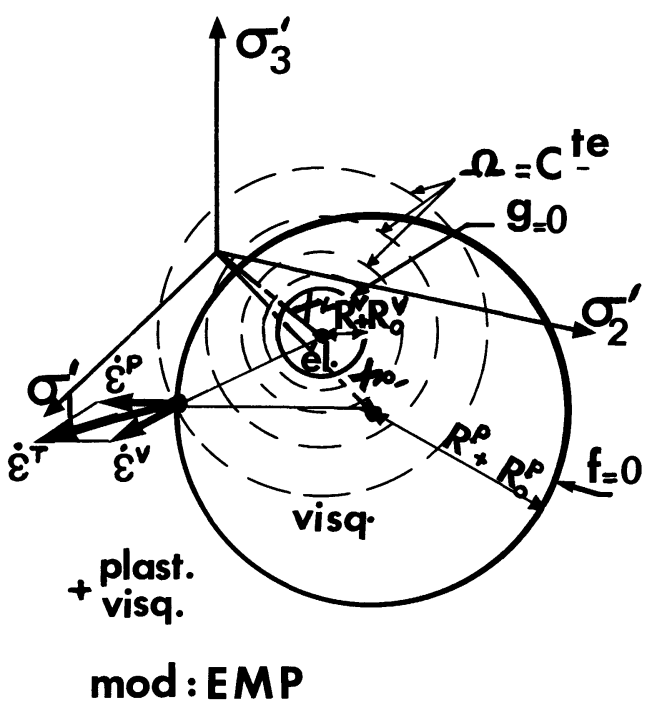

Fig. 15. - Schéma de principe du modèle de l'E.M.P.

[Basic sketch of the E.M.P. model.]

Dans l'espace des contraintes déviées (Fig. 15), il existe donc une surface seuil plastique, $f=0$, de rayon $R^{\mathrm{p}}$ translatée cinématiquement de $\underline{\mathbf{X}}^{\mathrm{p}^{\prime}}$, et dont l'écoulement plastique $\underline{\varepsilon}^{p}$ est normal. A l'exemple de la plasticité classique, le point de chargement $\underline{\mathbf{\sigma}}^{\prime}$ ne peut pas sortir de $f$ et l'on associe l'intérieur de cette surface au domaine d'écoulement visqueux (Fig. 15). Comme pour les modèles précédents, on définit des surfaces $\Omega$ d'égale dissipation et, à l'instar du modèle ONERA, homothétiques à une surface seuil élastique $g=0$, de centre $\underline{X}^{v^{\prime}}$ et de rayon $R^{v}$. La déformation visqueuse est déterminée au temps $t$ par la normale à l'équipotentielle de centre $\underline{\mathbf{X}}^{\mathbf{v}^{\prime}}$ et passant par $\underline{\mathbf{g}}^{\prime}$. Pour $g<0$, la déformation est élastique. De plus, la surface $g=0$ ne doit pas être extérieure à $f=0$, ce qui est réalisé en écrivant la relation de consistance $f=\dot{f}=0$ permettant de définir le multiplicateur $\dot{\lambda}$ de la déformation plastique (Eq. (19)). 
On peut résumer ce modèle comme suit ; existence d'une surface seuil plastique $f=0$, telle que :

$f=\left\langle J_{2}\left(\underline{\mathbf{\sigma}}-\underline{\mathbf{X}}^{\mathrm{p}}\right)-R^{\mathrm{p}}-R_{0}^{\mathrm{p}}\right\rangle+f_{\mathrm{r}}\left(R^{\mathrm{p}}\right)=0$,

et d'une surface seuil visqueuse $g=0$, dont l'équation réside dans :

$$
g=J_{2}\left(\underline{\underline{\sigma}}-\underline{\mathbf{X}}^{\mathrm{v}}\right)-R^{\mathrm{v}}-R_{0}^{\mathrm{v}}=0 .
$$

Le potentiel viscoplastique $\Omega$, homothétique à $g$ s'écrit donc :

$$
\Omega=\Omega_{\mathrm{v}}\left\langle J_{2}\left(\underline{\boldsymbol{\sigma}}-\underline{\mathbf{X}}^{\mathrm{v}}\right)-R^{\mathrm{v}}-R_{0}^{\mathrm{v}}\right\rangle+\Omega_{\mathrm{r}}\left(R^{\mathrm{v}}\right)=0 .
$$

Notons que dans la version originale du modèle, les variables cinématiques $\underline{\mathbf{X}}$ ne présentaient pas de terme de restauration, c'est-à-dire $\Omega_{\mathrm{r}}=f_{\mathrm{r}}=0$. Conformément à l'équation (19) traduisant la première normalité, la vitesse inélastique de déformation est donnée par :

$$
\dot{\varepsilon}_{i j}^{\mathrm{T}}=\dot{\lambda} \frac{\partial f(\ldots)}{\partial \sigma_{i j}}+\frac{\partial \Omega(\ldots)}{\partial \sigma_{i j}},
$$

avec $\dot{\lambda}$, multiplicateur plastique déterminé par la consistance

$$
f=\dot{f}=0 .
$$

Comme nous l'avons déjà mentionné, le couplage de comportement s'effectue au niveau des variables cinématiques $\underline{\mathbf{X}}^{\mathrm{v}}$ et $\underline{\mathbf{X}}^{\mathrm{p}}$. En effet, les définitions de $\underline{\mathbf{X}}^{v}$ et $\dot{\mathbf{X}}^{p}$ procèdent de la somme de deux cinétiques non linéaire à mémoire évanescente, le couplage s'effectuant par le coefficient $K^{\mathrm{vp}}$ (Eqs. (40) et (55f)). Une approche, cohérente, complètement découplée (en désaccord avec l'expérience) consiste simplement à prendre $K^{\mathrm{vp}}=0$. Les variables $\dot{R}^{\mathrm{p}}$ et $\dot{R}^{v}$, définissant les cinétiques d'évolution des rayons des surfaces $f$ et $g$, sont respectivement fonctions des déformations plastique et visqueuse cumulées, $\dot{p}$ et $\dot{v}$. On a donc le système :

$$
\left.\begin{array}{rl}
\dot{X}_{i j}^{\mathrm{p}}= & K^{\mathrm{p}} X^{\mathrm{p}}\left(\dot{\varepsilon}_{i j}^{\mathrm{p}}, \dot{p}, X_{i j}^{\mathrm{p}}, J_{2}\left(\underline{\mathbf{X}}^{\mathrm{p}}\right), T\right)+ \\
& +K^{\mathrm{vp}} X^{\mathrm{v}}\left(\dot{\varepsilon}_{i j}^{\mathrm{v}}, \dot{v}, X_{i j}^{\mathrm{v}}, J_{2}\left(\underline{\mathbf{X}}^{\mathrm{v}}\right), T\right) \\
\dot{X}_{i j}^{\mathrm{v}}= & K^{\mathrm{v}} X^{\mathrm{v}}\left(\dot{\varepsilon}_{i j}^{\mathrm{v}}, \dot{v}, X_{i j}^{\mathrm{v}}, J_{2}\left(\underline{\mathbf{X}}^{\mathrm{v}}\right), T\right)+ \\
& +K^{\mathrm{vp}} X^{\mathrm{p}}\left(\dot{\varepsilon}_{i j}^{\mathrm{p}}, \dot{p}, X_{i j}^{\mathrm{p}}, J_{2}\left(\underline{\mathbf{X}}^{\mathrm{p}}\right), T\right) \\
\dot{R}^{\mathrm{p}}= & R\left(\dot{p}, R^{\mathrm{p}}, T\right) \text { et } \dot{R}^{\mathrm{v}}=R\left(\dot{v}, R^{\mathrm{v}}, T\right)
\end{array}\right\}
$$

Il est intéressant d'observer que l'on retrouve les deux modèles précédents ainsi que leur version plastique indépendante du temps, moyennant quelques hypothèses sur les positions et les grandeurs relatives des surfaces $f$ et $g$. Ainsi, si $R^{\mathrm{p}}=R^{\mathrm{v}}$ et $X^{\mathrm{v}}=X^{\mathrm{p}}$, alors $f \equiv g$, les surfaces équipotentielles sont concentriques à $f=0$, il n'existe plus qu'une seule déformation inélastique et l'on retrouve le modèle associé unifié de l'ONERA-LMT Cachan. Par contre, si $R^{v}=0$ et si l'on ne considère que la déformation $\underline{\varepsilon}^{v}$, on se retrouve dans une situation proche de celle du modèle LMAB (à la notion de charge-décharge près). Les versions purement plastiques des deux modèles, avec $\underline{\mathbf{X}}^{\mathbf{v}^{\prime}}=\underline{\mathbf{\sigma}}^{\prime}$, sont identiques puisque $f \equiv F$.

On montre également l'analogie de ce modèle avec l'approche multicouche série du LMX, pour deux couches avec $(n)=(v)$ et $(n)=(p)$, mais avec des termes cinématiques non linéaires et les $R^{(n)}$ non constants.

5.1.2 Les modèles locaux structurels. - La philosophie des modèles multicouches (ou composites) a pour but de représenter l'hétérogénéité des états de déformation, de contrainte et de durcissement au sein d'un matériau polycristallin, chaque cristal étant d'orientation, de forme et d'état interne différent. Le passage des lois locales intrinsèque à chaque cristal (ou couche $(n)$ ) aux grandeurs globales correspondant au polycristal, peut s'effectuer de façon structurelle en considérant deux types d'assemblage, parallèle, c'est-à-dire $\underline{\varepsilon}^{n}=\underline{\varepsilon}$ (modèle CEA) ou série, $\underline{\mathbf{\sigma}}^{(n)}=\underline{\mathbf{\sigma}}$ (modèle du LMX). Nous examinons successivement ces deux types d'approches.

5.1.2.1 Modèle multicouche "parallèle ». du CEA $[33,34]$. - D'un point de vue international, on peut dire que les hypothèses fondamentales de ce modèle ont été émises par Besseling [44] puis reprises et exploitées intensivement au TNO, au Pays-Bas [45].

Pour chaque sous-matériau $(n)$, la formulation est simple et identique, du moins pour la variable déformation $\varepsilon_{i j}^{\mathrm{p}}$, à celle de la plasticité classique à écrouissage cinématique et isotrope (Fig. 16). Ainsi, pour chaque couche $(n)$, il existe, dans l'espace des contraintes déviées $\underline{\mathbf{\sigma}}^{(n)^{\prime}}$, une surface $f^{(n)}$ de centre $\underline{\mathrm{X}}^{\mathrm{p}(n)^{\prime}}$ et de rayon constant $R_{0}^{\mathrm{p}(n)}$, soit :

$$
f^{(n)}=J_{2}\left(\underline{\mathbf{\sigma}}^{(n)}-\underline{\mathbf{X}}^{\mathrm{p}(n)}\right)-R_{0}^{\mathrm{p}(n)}=0 .
$$

L'écoulement plastique est normal à cette surface, d'où, d'après l'équation (20) :

$$
\mathrm{d} \varepsilon_{i j}^{\mathrm{p}}=\mathrm{d} \lambda \lambda^{(n)} \frac{\partial f^{(n)}(\ldots)}{\partial \sigma_{i j}^{(n)}},
$$

$\mathrm{d} \lambda^{(n)}$ est le multiplicateur plastique qui indique que la composante $\underline{\boldsymbol{\sigma}}^{(n)}$ de la contrainte reste sur $f^{(n)}$, soit :

$$
f^{(n)}=\dot{f}^{(n)}=0
$$

Le type de déformation dans la couche $(n)$ est défini comme étant plastique (Eq. (56b)) si :

$$
J_{2}\left(\underline{\mathbf{\sigma}}^{(n)}-\underline{\mathbf{X}}_{0}^{\mathrm{p}(n)}\right)>R_{0}^{\mathrm{p}(n)}
$$

et élastique (avec les mêmes constantes élastiques 


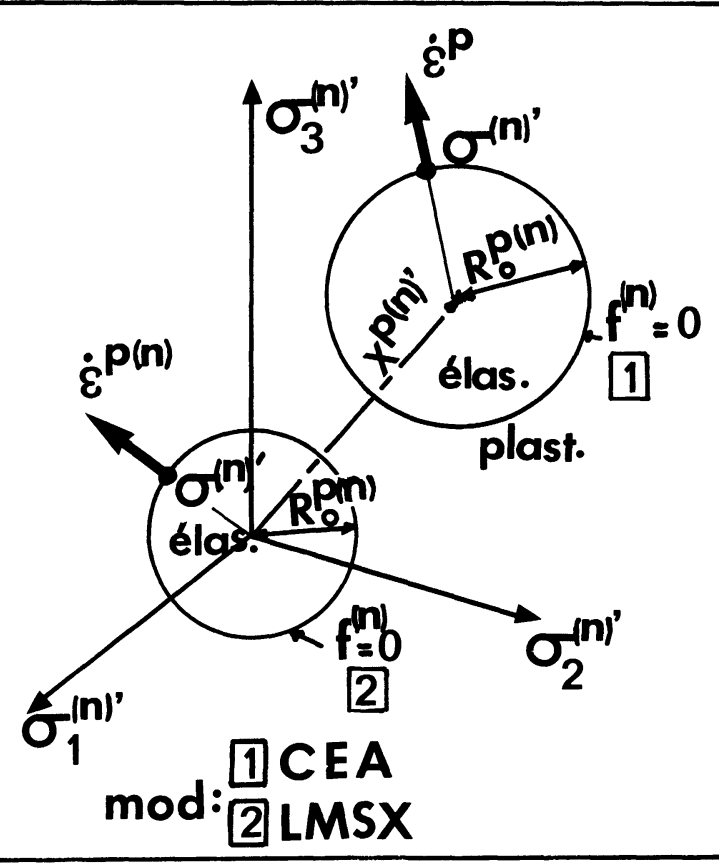

Fig. 16. - Schéma de principe des modèles multicouches CEA et LMX.

[Basic sketch of the multilayers CEA and LMX models.]

pour chaque sous-matériau) dans le cas contraire ; $J_{2}\left(\underline{\sigma}^{(n)}-\underline{\mathbf{X}}_{0}^{(n)}\right)<R_{0}^{\mathrm{p}(n)} \cdot \underline{\mathbf{X}}_{0}^{\mathrm{p}(n)}$ est la valeur de $\underline{\mathbf{X}}^{\mathrm{p}}$ atteinte à la dernière extrémité du cycle. Cependant, dans la version originale (hybride) de ce modèle, le facteur temps intervient par l'intermédiaire des paramètres de restauration agissant sur chacune des variables cinématiques. Ainsi, dans l'optique des auteurs, restauration et écoulement visqueux constituent deux mécanismes distincts, fonction du temps et affectant la déformation inélastique. Dans les trois modèles précédents, viscosité et restauration sont toujours liées et l'on montrera ultérieurement qu'une version purement visqueuse du modèle CEA est tout à fait réaliste.

Pour introduire la restauration sur chacune des couches, indépendamment de la viscosité, il suffit de postuler l'existence de potentiels de restauration $\Omega^{(n)}$, tels que $\Omega^{(n)}=\Omega_{\mathrm{r}}^{(n)}\left(J_{2}\left(\underline{X}^{\mathrm{p}}\right), T\right)$. Puisque $\Omega^{(n)}$ n'a pas de terme visqueux $\Omega_{\mathrm{v}}^{(n)}$, à l'inverse des modèles précédents, ce potentiel n'affecte pas la nature plastique de l'écoulement (Eq. (20) et (56b)), $\underline{\boldsymbol{\sigma}}^{(n)}$ reste sur $f^{(n)}$. Ainsi, les variables internes s'écriront :

$$
\left.\begin{array}{l}
\dot{X}_{i j}^{\mathrm{p}(n)}=X^{\mathrm{p}}\left(\dot{\varepsilon}_{i j}, X_{i j}^{\mathrm{p}}, J_{2}\left(X^{\mathrm{p}}\right), T\right) \\
R^{\mathrm{p}(n)}=R_{0}^{\mathrm{p}(n)}
\end{array}\right\} .
$$

Les cinétiques $\dot{X}_{i j}^{p(n)}$ sont linéaires en déformation (écrouissage de Prager) avec restauration par le temps.
Il reste à écrire les équations d'équilibre du système, c'est-à-dire passer des grandeurs locales $\underline{\mathbf{\sigma}}_{\ell}$ aux valeurs moyennes globales $\underline{\mathbf{\sigma}}$, soit : $\underline{\mathbf{\sigma}}=$ $\frac{1}{v} \int_{v} \underline{\mathbf{\sigma}}_{\ell} \mathrm{d} v$, que l'on discrétise à l'aide des $(n)$ couches :

$$
\underline{\mathbf{\sigma}}=\frac{1}{v} \sum_{(n)} v^{(n)} \underline{\mathbf{\sigma}}^{(n)}=\sum_{(n)} w^{(n)} \underline{\mathbf{\sigma}}^{(n)} .
$$

$w^{(n)}$ est tel que $\sum_{(n)} w^{(n)}=1$ et représente le volume relatif (ou le poids) du sous-matériau $(n) . \underline{\mathbf{\sigma}}^{(n)}$ se déduit de l'équation de consistance $(56 \mathrm{c})$, soit $\left(\dot{\sigma}^{(n)}-\dot{X}^{\mathrm{p}(n)}\right)\left(\sigma^{(n)}-X^{\mathrm{p}(n)}\right)=0$, qui en uniaxial permet d'aboutir à la solution simple: $\sigma=$ $\sum_{(n)} w^{(n)}\left(X^{\mathrm{p}(n)}+R^{\mathrm{p}(n)}\right)$. L'organigramme de ce modèle réside donc dans les équations (56), et l'on voit dès à présent que le paramètre $(n)$, indéterminé, est décisif dans ce type d'approche.

5.1.2.2 Modèle multicouche «série » du LMX [3537]. - Contrairement au modèle précédent, on suppose que la déformation globale $\underline{\varepsilon}^{p}$ est la moyenne de la somme des déformations plastiques locales, d'où la terminologie de «série », soit : $\underline{\boldsymbol{\varepsilon}}^{\mathrm{p}}=\frac{1}{v} \int_{v} \mathbf{B}^{\mathrm{T}} \underline{\boldsymbol{\varepsilon}} \mathfrak{\mathrm { d }} v$, et en discrétisant à $(n)$ couches :

$$
\underline{\varepsilon}^{\mathrm{p}}=\mathbf{B}^{\mathrm{T}} \sum_{(n)} w^{(n)} \underline{\varepsilon}^{\mathrm{p}(n)}
$$

$\mathbf{B}^{\text {I }}$ est la transposée de $\mathbf{B}$ qui est une matrice élastique dont la définition est donnée en (57b). La seconde hypothèse est que la contrainte locale $\underline{\mathbf{\sigma}}_{\ell}$ s'exprime sous la forme :

$$
\underline{\boldsymbol{\sigma}}_{\ell}=\mathbf{B} \underline{\boldsymbol{\sigma}}-\underline{\boldsymbol{\rho}},
$$

où $\underline{\rho}$ représente le tenseur des contraintes résiduelles résultant des incompatibilités plastiques, c'est-à-dire qu'en absence de plasticité, contraintes locale et globale sont liées par la relation, $\underline{\mathbf{\sigma}}_{\ell}=\mathbf{B} \underline{\mathbf{\sigma}}$, d'où la définition de la matrice d'élasticité $\mathbf{B}$. Le problème consiste donc à connaître le comportement local et à évaluer dans chaque sous-matériau la matrice $\mathbf{B}$ et le tenseur $\boldsymbol{\rho}$.

Un cas simple de ce modèle souvent utilisé pour les matériaux initialement isotrope, est de considérer $\mathbf{B}=$ I (matrice unité), c'est-à-dire qu'élastiquement, les contraintes locale et globale sont égales. Dans ce cas,

$$
\underline{\boldsymbol{\varepsilon}}^{\mathrm{p}}=\sum_{(n)} \underline{\boldsymbol{\varepsilon}}^{\mathrm{p}(n)}
$$

et localement les contraintes déviées peuvent s'écrire :

$$
\sigma_{i j}^{(n)^{\prime}}=\sigma_{i j}^{\prime}-X_{i j}^{\mathrm{p}(n)^{\prime}}
$$


où $\underline{\underline{X}}^{\mathrm{p}^{(n)}}$ est identique au tenseur des contraintes résiduelles $\boldsymbol{\rho}$. Il s'agit maintenant de définir les lois de comportement plastique locales (ou de chaque sous-couche) et à l'exemple de la plasticité classique (Fig. 16), on prend des fonctions seuils du type :

$$
f^{(n)}=J_{2}\left(\underline{\boldsymbol{\sigma}}^{(n)}\right)-R_{0}^{\mathrm{p}(n)}=0 ，
$$

soit d'après $(57 d)$ :

$$
f^{(n)}=J_{2}\left(\underline{\mathbf{\sigma}}-\underline{\mathbf{X}}^{\mathrm{p}(n)}\right)-R_{0}^{\mathrm{p}^{(n)}}=0 .
$$

En appliquant la première hypothèse de normalité, on retrouve l'équation (21) donnant les déformations plastiques :

$$
\mathrm{d} \varepsilon_{i j}^{(n)}=\mathrm{d} \lambda{ }^{(n)} \frac{\partial f^{(n)}(\ldots)}{\partial \sigma_{i j}} .
$$

Le multiplicateur plastique $\lambda^{(n)}$, exprimant que $\underline{\text { o }}$ reste $\operatorname{sur} f^{(n)}=0$, est donné par :

$$
f^{(n)}=\dot{f}^{(n)}=0 \text {. }
$$

Pour ce qui concerne les lois d'évolution des variables cinématiques locales, les auteurs adoptent des relations linéaires de type Prager :

$$
\dot{\mathbf{x}}^{\mathrm{p}}=\mathbf{A} \underline{\dot{\varepsilon}}^{\mathrm{p}},
$$

soit :

$$
\dot{X}_{l j}^{\mathrm{p}(m)}=\sum_{\ell=1}^{(n)} a_{m \ell}^{\mathrm{p}} \dot{\varepsilon}_{i j}^{\mathrm{p}(\ell)}, \quad m \in[1, n],
$$

avec $a_{m \ell} \geqq 0$ et $a_{m \ell}=a_{\ell m}$ (Eq. (29)). De plus, pour chaque surface $f^{(n)}$ son rayon est considéré comme constant et égal à $R_{0}^{\mathrm{p}(n)}$. Les équations $(57 \mathrm{c}$ à h) réalisent une version entièrement plastique du modèle du LMX puisqu'il n'apparaît aucun terme fonction du temps. Comme pour le modèle précédent, une version entièrement viscoplastique est facilement envisageable et le choix de (n) est également décisif. On peut mentionner une analyse générale de ce modèle fixant ses degrés de liberté par rapport aux phénomènes de rochet cycliques pour un état de contrainte appliquée biaxé, avec $(n)=2$ et une matrice $\mathbf{B}$ complète, de dimension deux, pour chaque sous-couche [37]. On note également que le caractère multi-surface de ce modèle est similaire à celui de la version première de (1967) du modèle de Mroz.

\subsection{JUSTIFICATION PHYSIQUE ET EXPÉRIMENTALE DU CHOIX DES DIFFÉRENTS POTENTIELS $f$ ET/OU $\Omega$ ASSOCIÉS AUX LOIS D'ÉCOULEMENT.}

5.2.1 Les prémisses physiques. - Dans les trois premiers modèles, le point de chargement ( $\underline{\mathbf{\sigma}})$ est toujours extérieur à $f, g$ ou $F$, ce qui implique qu'en 1D (pour simplifier), on peut définir une composante visqueuse $\sigma_{\mathrm{v}}$ par :

$$
\sigma_{\mathrm{v}}=\sigma-\sigma_{\mathrm{i}}
$$

où $\sigma_{\mathrm{i}}$ représente la contrainte interne moyenne qui s'oppose au mouvement des dislocations mobiles.

A ce niveau, il semble souhaitable de préciser à l'aide d'un exemple simple, le sens physique des notions de contrainte interne et d'état local. Considérons une dislocation isolée dans son plan de glissement contenant des obstacles interagissant à courte distance avec la dislocation (défauts ponctuels, précipités, ...) et faisons un bilan des forces qui lui sont appliquées. Sous l'action de la contrainte extérieure $\sigma$ (projetée dans le plan et la direction de glissement $x$ ) le segment de dislocation de longueur $l$ est soumis à une force $F(F=\sigma b l)$ ainsi qu'à une force interne $F_{\mathrm{i}}$, opposée à la précédente et associée à l'ensemble des défauts du cristal agissant à grande distance (forêt de dislocations, parois, échevaux, ...). Notons que $F_{\mathrm{i}}$ ne varie spatialement que très lentement et peut être considérée comme constante sur une faible distance; en particulier sur $x_{0} x_{\mathrm{c}}$ qui représente la distance franchie par la dislocation suivant $x$ entre deux positions d'équilibre. Les obstacles à courte distance exercent également une force $F_{\mathrm{b}}$ intrinsèque à la nature de l'interaction dislocation-obstacle. Dans ce contexte, l'accroissement $\Delta G$ de l'énergie libre du système, pour un isotherme donné, entre les deux positions d'équilibre peut en première approximation s'écrire :

$$
\Delta G=\int_{x_{0}}^{x_{\mathrm{c}}}\left(F_{\mathrm{b}}(x)+F_{\mathrm{i}}(x)-F\right) \mathrm{d} x .
$$

Si maintenant le profil de l'interaction $F_{\mathrm{b}}(x)$ est indépendant de la contrainte appliquée, $\Delta G$ n'est fonction de la contrainte que par l'intermédiaire des bornes $x_{0}$ et $x_{c}$ et du travail effectué par cette dernière pendant l'activation d'un événement.

Ainsi, on peut associer à $x_{0}$ et $x_{\mathrm{c}}$ des fonctions d'une force fictive $F_{\mathrm{v}}=F-F_{\mathrm{i}}$, à laquelle on fera correspondre une contrainte effective (ou visqueuse), $\sigma_{\mathrm{v}}=\sigma-\sigma_{\mathrm{i}}$, où $\sigma_{\mathrm{i}}$ représente la contrainte interne. Si l'on pose $\int_{x_{0}}^{x_{\mathrm{c}}} F_{\mathrm{b}} \mathrm{d} x=\Delta g ; X=x_{0} x_{\mathrm{c}}$ et si $\sigma_{\mathrm{v}}$ est indépendante de la contrainte, on peut réécrire l'accroissement d'énergie libre sous la forme :

$$
\Delta G=\Delta g-\sigma_{\vee} b l X
$$

Cette expression montre qu'il est possible de traiter le problème thermodynamiquement en utilisant comme système isolé un petit élément de volume de matière englobant les obstacles à courte distance et le segment de dislocation, l'ensemble des forces extérieures à ce système étant représenté par la contrainte effective $\sigma_{\mathrm{v}}$. Cet exemple simple illustre physiquement les concepts de contrainte interne et d'état local qui sont, comme nous l'avons évoqué précédemment, à la base de l'écriture thermodynamique des modèles présentés. 
L'étude de la viscosité d'un matériau repose donc sur la connaissance de la relation $\dot{\varepsilon}=f\left(\sigma-\sigma_{\mathrm{i}}\right)=$ $f\left(\sigma_{\mathrm{v}}\right)$. En plasticité, $\sigma_{\mathrm{v}}=0$ puisque $\sigma=\sigma_{\mathrm{i}}$ et $\dot{\varepsilon}=\dot{\lambda}$, avec $\dot{\lambda}$ multiplicateur plastique déterminé par une relation de consistance. Rappelons que $\sigma_{i}$ représente la somme de diverses contributions qui correspondent localement aux différents types d'interactions microscopiques recensées par les métallurgistes, c'est-à-dire pour les températures intermédiaires, les interactions dislocations-dislocations à courte et longue distance, dislocations-précipités et dislocations-défauts ponctuels à courte distance. Ces différentes contributions peuvent être de nature scalaire ou tensorielle.

Pour les modèles ONERA et EMP, d'après les équations (53a) et (53b), on peut écrire pour un chargement monotone $1 \mathrm{D}$ :

$$
\sigma_{\mathrm{i}}=X^{\mathrm{v}}+R^{\mathrm{v}}+R_{0}^{\mathrm{v}}
$$

et pour le modèle LMAB (Eq. (54a), (54e) et (54f)),

avec

$$
\begin{gathered}
\sigma_{\mathrm{i}}=X^{\mathrm{v}} \\
X^{\mathrm{v}}=\sum_{1}^{2} X^{\mathrm{vM}}=X^{\mathrm{vM}(2)}+R^{\mathrm{v}}
\end{gathered}
$$

dans l'état de charge et :

$$
X^{\mathrm{v}}=X^{\mathrm{vm}}
$$

pour une décharge. En ce qui concerne les modèles multicouches CEA et $\mathrm{LMX}$, on a respectivement :

$$
\begin{gathered}
\sigma_{\mathrm{i}}=\sigma=\sum_{(n)} w^{(n)}\left(X^{\mathrm{p}(n)}+R_{0}^{\mathrm{p}(n)}\right) \\
\sigma_{\mathrm{i}}=\sigma=X^{\mathrm{p}(n)}+R_{0}^{\mathrm{p}(n)} .
\end{gathered}
$$

et

On remarque la cohérence de ces formules et dès lors il semble possible, pour l'ensemble de ces modèles, de corréler chaque type d'interaction à une variable bien précise. On retrouve à ce niveau la différence entre les approches globales et multicouches et qui réside dans le traitement des variables $X$ et $R$ sous une forme continue ou discrète. Dans les deux types d'approches, la nature physique de ces variables est identique, seul le degré d'homogénéisation lié à la taille de la cellule considérée, change.

Généralement, on attribue $\underline{\mathbf{X}}$ (écrouissage cinématique), tenseur du second ordre, aux interactions dislocations-dislocations à longue distance, créant une contrainte en retour induite par les inhomogénéités des déformations plastiques entre grains. De même, pour les matériaux monophasés avec précipités ou biphasés, $\underline{\mathbf{X}}$ correspond aux interactions dislocations-précipités/(ou seconde phase), le niveau de $\underline{\mathbf{X}}$ étant lié au degré de cohérence entre matrice et précipités. Ainsi, une fine dispersion de précipités très durs, incohérents avec la matrice, augmente considérablement le niveau de $\underline{\mathbf{X}}$ et favorise la tenue en fluage, ce qui est présentement le cas du superalliage INCO 718 avec les précipités $\gamma^{\prime}$ et $\gamma^{\prime \prime}$.

$R$ est une variable scalaire directement liée aux variations isotropes de la densité totale de dislocation et fonction des configurations prises par ces dernières, à savoir, la formation de cellules ou de parois de polygonisation de taille et d'épaisseur variables. $R_{0}$ désigne la valeur initiale de $R$, correspondant à la limite élastique initiale du matériau ou de chaque grain (modèles multicouches), et associée à la densité initiale de dislocations ou précipités après recuit. Avec une telle définition, on a donc $R(0)=0$ au temps $t=0$, alors que pour le modèle LMAB où il n'apparaît pas explicitement de terme $R_{0}$, on a $R(0)=R_{0}$, et l'état initial du matériau dépend de l'histoire antérieure et particulièrement du traitement thermomécanique subi par l'échantillon. Cependant, pour l'ensemble des modèles, hormis celui du LMAB, $R$ et $\underline{\mathbf{X}}$ sont des variables (ou paramètres) indépendantes, ce qui paraît difficilement justifiable au regard de la nature physique qui vient de leur être attribuée. Dans le modèle LMAB, comme on peut le voir d'après les équations $(51), R$ est un paramètre scalaire évolutif (norme de $X_{i j}^{\mathrm{vM}(1)}$ ), qui prend la même signification que celle donnée jusqu'à présent, mais qui conditionne, dans l'état de charge c'est-à-dire de création de la sous-structure, l'évolution des deux variables cinématiques $\underline{\underline{X}}^{\mathrm{vM}(1)}$ et $\underline{\mathbf{X}}^{\mathrm{vM}(2)}$. Dans cette optique, $\underline{\mathbf{X}}^{\mathrm{vM(1)}}$ peut être interprétée comme une composante de la contrainte en retour liée à la formation des cellules ou parois, durant un chargement cyclique par exemple, et $\underline{\mathbf{X}}^{\mathrm{vM(2)}}$ comme la composante due aux dislocations d'incompatibilité plastique aux joints de grains, ces deux composantes étant fonction des variations de la densité totale des dislocations représentée par $R$. Comme nous le verrons ultérieurement, les paramètres de mémorisation introduits dans les différents modèles (hormis les multicouches) et agissant sur $R$, peuvent être associés aux paramètres géométriques de la sous-structure développée, par exemple la taille, le degré de désorientation des cellules, ..., qui présentent fréquemment des mémoires de forme liées aux états de déformation ou de contrainte.

Les interactions à courtes distances, dislocationsdéfauts ponctuels substitutionnels ou interstitiels caractéristiques d'effets d'instabilité tel que le phénomène Portevin-Le Chatelier, ou de perte de viscosité souvent associée à un maximum sur la contrainte d'écoulement [46, 47], peuvent être prises en compte par l'introduction d'une nouvelle variable isotrope $R^{*}$ qui représente la somme des contributions de chacune des interactions entre défaut-ponctuel bien précis et dislocations. Ce point, très conséquent dans le cas du $17-12 \mathrm{SPH}$ entre 20 et $525^{\circ} \mathrm{C}$ est en cours d'étude dans le cas du modèle LMAB. D'une manière générale $R^{*}$ peut intervenir sous des formes additive ou multiplicative par rapport aux autres 
variables, mais pour les mêmes raisons que précédemment, la seconde solution semble physiquement plus raisonnable et $R^{*}$ devrait être liée à $R$, ce type d'interaction étant directement fonction de la densité totale des dislocations donc fonction de la déformation. Ce point important sera évoqué ultérieurement dans le paragraphe consacré au développement futur de ces modèles.

A ce stade, il semble intéressant d'évoquer le modèle de Krempl [48, 49] qui repose sur l'existence d'une contrainte d'équilibre $g(\varepsilon)$ (overstress) correspondant à la courbe effort-déformation lorsque la vitesse de déformation est infiniment lente. Signalons qu'à notre connaissance, cette idée a initialement été émise par Philipps [50]. En uniaxial, ce modèle s'écrit :

$$
\dot{\varepsilon}-\frac{\dot{\sigma}}{E}=\frac{\sigma-g(\varepsilon)}{E K(\sigma-g(\varepsilon))},
$$

où $E$ est le module d'Young et $K$ une fonction à déterminer. Si l'on pose $F(u)=\frac{u}{E K(u)}$ et que l'on inverse (63), on arrive à :

$$
\sigma=g(\varepsilon)+F^{-1}\left(\dot{\varepsilon}-\frac{\dot{\sigma}}{E}\right)
$$

que l'on doit comparer à (58). Ainsi, la fonction d'équilibre $g(\varepsilon)$ est identique à $\sigma_{i}$ et peut prendre la même signification physique, alors que $F^{-1}\left(\dot{\varepsilon}-\frac{\dot{\sigma}}{E}\right)$ joue le rôle de la composante visqueuse $\sigma_{\mathrm{v}}$. Pour un chargement monotone, le modèle de Krempl est très proche de ceux qui viennent d'être évoqués et ainsi, fluage et relaxation sont naturellement pris en compte, et en l'absence de restauration doivent se terminer quand la déformation ou la contrainte, arrive sur la courbe d'équilibre. Puisqu'il s'agit d'une théorie isotrope évoluant en dehors du concept des variables internes, l'analogie avec les modèles présentés se limite aux essais monotones. En effet, pour tenir compte des retours en arrière et des aspects cycliques, le modèle de Krempl a recours à une procédure de mise en mémoire et de réinitialisation qui consiste à mémoriser l'instant où la contrainte franchit la courbe d'équilibre et à modifier simultanément $g(\varepsilon)$.

On doit également signaler l'hypothèse de départ du modèle développé à l'IMG [4] et qui consiste à additionner les contributions à la contrainte de trois phénomènes dissipatifs fondamentaux, à savoir, le frottement solide relevant d'une description à mémoire discrète $\left(\sigma_{\mathrm{a}}\right)$, le changement de structure dépendant de la vitesse de déformation, ou frottement visqueux, relevant d'une mémoire continue $\left(\sigma_{v}\right)$ et enfin le changement de structure indépendant de la vitessé de déformation possédant une mémoire continue $\left(\sigma_{\mathrm{g}}\right)$. On postule donc que : $\sigma=\left(\sigma_{\mathrm{a}}+\sigma_{\mathrm{g}}\right)+\sigma_{\mathrm{v}}(64)$, identique à (58) avec $\sigma_{\mathrm{i}}=\sigma_{\mathrm{a}}+\sigma_{\mathrm{g}}$ et qui représente la contribution indépendante de la vitesse de déformation. Comme pour le modèle de Krempl, l'analogie ne va pas au-delà puisque les évolutions $\dot{\sigma}_{\mathrm{a}}$ et $\dot{\sigma}_{\mathrm{g}}$ sont liées à des algorithmes qui sélectionnent une suite de points particuliers mémorisables, par exemple la contrainte atteinte au dernier point d'inversion associé au paramètre de Masing $\omega=1$ ou 2. Au niveau des algorithmes de mémorisation de l'inversion, on peut noter une certaine analogie avec la théorie endochronique initialement développée par Valanis [51].

En conclusion, on peut dire que les prémisses physiques des nombreux modèles évoqués sont identiques et reposent sur l'équation (58). Par contre, les différences essentielles apparaissent au niveau du traitement de $\sigma_{i}$ d'une manière continue (modèles unifiés) ou discrète (multicouches) et de la description des réponses aux inversions du chargement, qui s'opère d'une façon continue ou discrète, sans mémorisation (modèles à variables internes) ou avec mémorisation de points particuliers du trajet de chargement (modèles de Krempl, de l'IMG ou théorie endochronique, entr'autres).

5.2.2 Détermination de la composante visqueuse $\sigma_{\mathrm{v}}$. - Pour appréhender $\sigma_{\mathrm{v}}$ et par voie de conséquence $\sigma_{\mathrm{i}}=\sigma-\sigma_{\mathrm{v}}$, il n'existe, à notre connaissance, qu'une seule technique expérimentale qui a été fort discutée par les physiciens du solide [52-54] mais donc l'objectivité et la validité semblent avoir été démontrées $[55,56]$, et qui réside dans la méthode des brusques décréments de contrainte ou de déformation durant un essai de fluage [57] ou lors d'un essai à vitesse imposée [58, 59]. L'hypothèse fondamentale sous-jacente à cette technique est que l'on admet que la structure du matériau reste figée pendant quelques secondes, ce qui permet d'effectuer une mesure avant qu'un retour vers un nouvel équilibre s'établisse. On doit mentionner qu'il existe des cas, très hautes températures, fortes contraintes et grandes perturbations, où cette hypothèse n'est plus vérifiée, la variable interne (structure) évoluant presque aussi rapidement que les variables externes (contrainte ou de déformation). Ainsi, durant un essai de traction à vitesse imposée, on réalise successivement des décréments de déformation $\Delta \varepsilon^{\mathrm{T}}$ croissants et l'on mesure les vitesses de relaxation instantanées $\dot{\sigma}_{z z i}$ (temps de mesure de l'ordre de 20 à $30 \mathrm{~s}$ ) qui en résultent. Celles-ci peuvent être positives, nulles ou négatives et le niveau de contrainte interne moyen $\sigma_{i}$ est obtenu pour $\dot{\sigma}_{z z i}=0$. La méthode complémentaire s'applique durant un essai de fluage et c'est la vitesse de fluage instantanée $\dot{\varepsilon}_{z z i}$, après décrément de contrainte $\Delta \sigma_{z z}$, qui permet d'obtenir la valeur de $\sigma_{\mathrm{i}}$, lorsque $\dot{\varepsilon}_{z z i}=0$ pour un certain décrément $\Delta \sigma_{z z}^{*}$, on a : $\sigma_{\mathrm{i}}=\Delta \sigma_{z z}^{*}$ donc $\sigma_{\mathrm{v}}=\sigma_{z z}-\Delta \sigma_{z z}^{*}$.

Une extension de cette technique au cas des 
chargements radiaux de traction-torsion a été réalisée par Delobelle et coll. [27, 40] et permet d'accéder aux deux composantes $\left\{X_{z z}, X_{z \vartheta}\right\}$ de la contrainte interne et qui correspondent respectivement, pour un essai de fluage, aux coordonnées :

$$
\frac{\dot{\varepsilon}_{z \vartheta \mathrm{i}}}{\dot{\varepsilon}_{z z \mathrm{i}}}=0 \quad \text { et } \quad \frac{\dot{\varepsilon}_{z \vartheta \mathrm{i}}}{\dot{\varepsilon}_{z z \mathrm{i}}}=\infty \text {. }
$$

Un exemple a été présenté sur la figure $14 \mathrm{~b}$. Cette méthode permet donc de corréler $\overline{\dot{\varepsilon}}$ avec $J_{2}\left(\underline{\sigma}-\underline{X}^{\mathbf{v}}\right)$ et en uniaxé $\dot{\varepsilon}_{z z}=f\left(\sigma_{\mathrm{v}}\right)$.

Dans cette optique, une campagne extensive a été menée sur le 17-12 SPH [7,9] et l'on reporte sur la figure 17 (courbe (1)), en coordonnées logarithmiques, l'ensemble des points expérimentaux, $\overline{\dot{\varepsilon}}=f\left(\sigma_{\mathrm{v}}\right)$, pour $T=600^{\circ} \mathrm{C}$, déterminés d'une part, durant des essais de fluage uniaxé ou biaxé pendant les phases primaire ou stationnaire de l'écoulement, et d'autre part, pour des essais monoaxés à vitesse imposée (traction ou torsion pure). Le fait le plus marquant est la très forte non-linéarité de cette

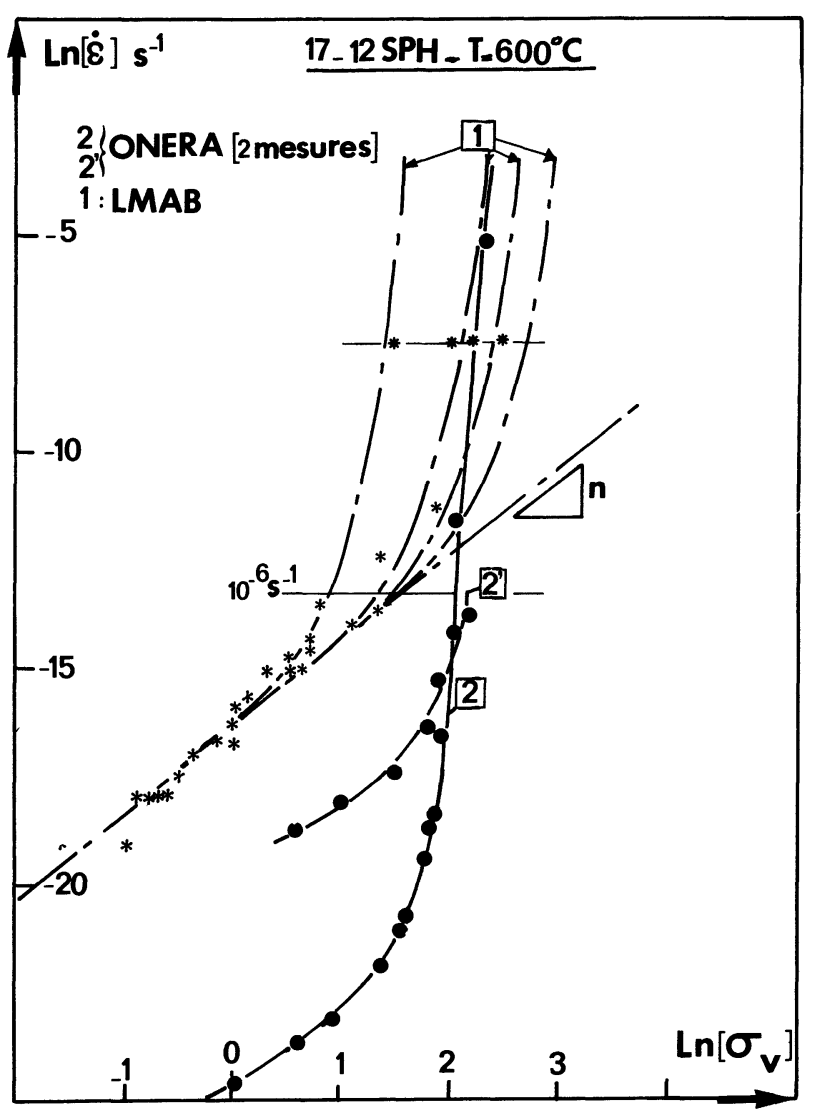

Fig. 17. - Etude de la relation $\dot{v}=f\left(\sigma_{\mathrm{v}}\right)$. Mise en évidence de la très forte non-linéarité. (D'après Nouailhas [17] avec l'amabilité de l'ONERA et Delobelle [7]).

[Study of the $\dot{v}=f\left(\sigma_{\mathrm{v}}\right)$ relationship. Demonstration of a strong non linearity. (From Nouaihas [17] with courtesy of ONERA and from Delobelle [7]).] représentation, c'est-à-dire que l'exposant $n^{*}$ de la loi de Norton écrite avec la composante visqueuse, $\dot{\varepsilon}=\dot{\varepsilon}_{0} \sigma_{\mathrm{v}}^{n^{*}}$, croît de 2 à 25 avec $\sigma_{\mathrm{v}}$. Les déterminations indirectes de $\sigma_{\mathrm{v}}$, réalisées à l'ONERA (courbes (2) ) à partir d'essais de relaxation et de fluage [17] confirment ce point capital. Comme nous le verrons ci-dessous, on comprend mieux le modèle partitionné qui consiste à traduire cette non-linéarité en prenant deux valeurs extrêmes de $n^{*}$, soit $n^{*}=\infty$ dans le cas de la plasticité et $n *$ fini pour la viscosité. Cependant, si pour les grandes vitesses de déformation les courbes (1) et (2) sont assez proches, pour les plus faibles vitesses $\left(\dot{\varepsilon} \leqq 10^{-6} \mathrm{~s}^{-1}\right)$, la perte de linéarité sur $n^{*}$ se produit plus tôt sur la courbe (1). Ce constat montre que si, qualitativement, il y a consensus sur la perte de viscosité avec la vitesse, les désaccords quantitatifs restent à préciser et analyser, du moins numériquement. Avec une telle non-linéarité les courbes effort-déformation sont peu sensibles à la vitesse, mais par contre, les courbes de fluage doivent présenter un coude très marqué correspondant à la chute rapide de l'exposant $n^{*}$, ce qui est conforme à l'expérience (Fig. 2). Une autre constatation importante est qu'il semble exister une relation bi-univoque, $\overline{\dot{\varepsilon}}=f\left(\sigma_{\mathrm{v}}\right)$, durant le fluage après la déformation de mise en charge, lorsque les vitesses deviennent faibles $\left(\dot{\varepsilon} \leqq 10^{-6} \mathrm{~s}^{-1}\right.$, Fig. 17, courbe (1) ) et qu'à l'opposé, pour les plus grandes vitesses et particulièrement pour les essais à vitesse imposée $\left(\dot{\varepsilon}^{\mathrm{T}} \geqq 10^{-5} \mathrm{~s}^{-1}\right)$ cette relation n'est plus unique. Cet effet est illustré sur la figure 18 dans le cas d'un acier 17-12 $\mathrm{SPH}$ pour $\dot{\varepsilon}^{\mathrm{T}}=6,6 \times 10^{-4} \mathrm{~s}^{-1}$, où l'on montre que $\sigma_{\mathrm{v}}$ croît avec la déformation et varie bien évidemment avec la température, c'est-à-dire que :

$$
\sigma_{\mathrm{v}}=\sigma_{\mathrm{v}}(\overline{\dot{\varepsilon}}, \bar{\varepsilon}, T) \text {. }
$$

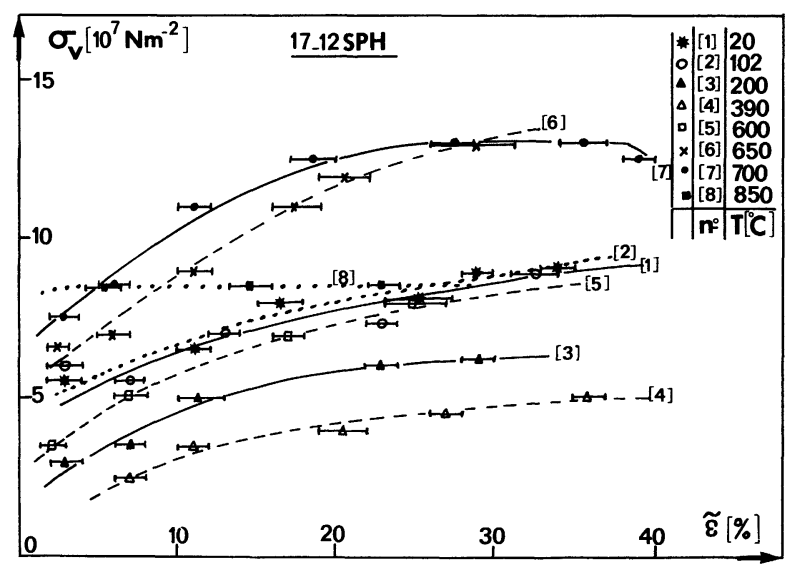

Fig. 18. - Mise en évidence de la dépendance de $\sigma_{\mathrm{v}}$ avec la déformation pour différentes températures. On note que pour un écoulement stationnaire (courbes 7 et 8 ) $\sigma_{\mathrm{v}}$ ne dépend plus de $\varepsilon$. (D'après Delobelle [47]).

[Evidence of the dependency of $\sigma_{v}$ on strain for various temperatures. For steady creep (curves 7 and 8), note that $\sigma_{v}$ no longer depends on $\varepsilon$. (From Delobelle [47]).] 
De plus, l'analyse de la relaxation au cours d'un temps de maintien au maximum d'un cycle (pour un nombre de cycles fixé) en fonction de l'amplitude de cyclage imposée $\left(\frac{\Delta \varepsilon^{T}}{2}\right)$, révèle que l'amplitude relaxée augmente avec $\left(\frac{\Delta \varepsilon^{\mathrm{T}}}{2}\right)$, ce qui peut effectivement s'interpréter comme l'augmentation de $\sigma_{v}$ avec $\left(\frac{\Delta \varepsilon^{T}}{2}\right)$. Cet aspect est présent dans les deux modèles unifiés ONERA-LMT et LMAB. Au regard des résultats expérimentaux présentés, on comprend qu'une version viscoplastique des modèles multicouches CEA, LMX, soit nécessaire.

Toutes ces conclusions ont été acquises sur le 17$12 \mathrm{SPH}$ et confirmées sur quelques alliages légers [27], mais par contre, on doit constater un manque de données expérimentales sur les phénomènes de viscosité dans l'INCO 718.

5.2.3 Lois de déformation et formulation des différents potentiels: exemple du 17-12 SPH. - On illustre schématiquement sur la figure 19 les différentes approches pour appréhender la forte non-linéarité de la relation $\operatorname{Ln} \dot{v}=f\left(\operatorname{Ln} \sigma_{v}\right)$. On examine successivement chaque modèle et une extension aux modèles multicouches est présentée.

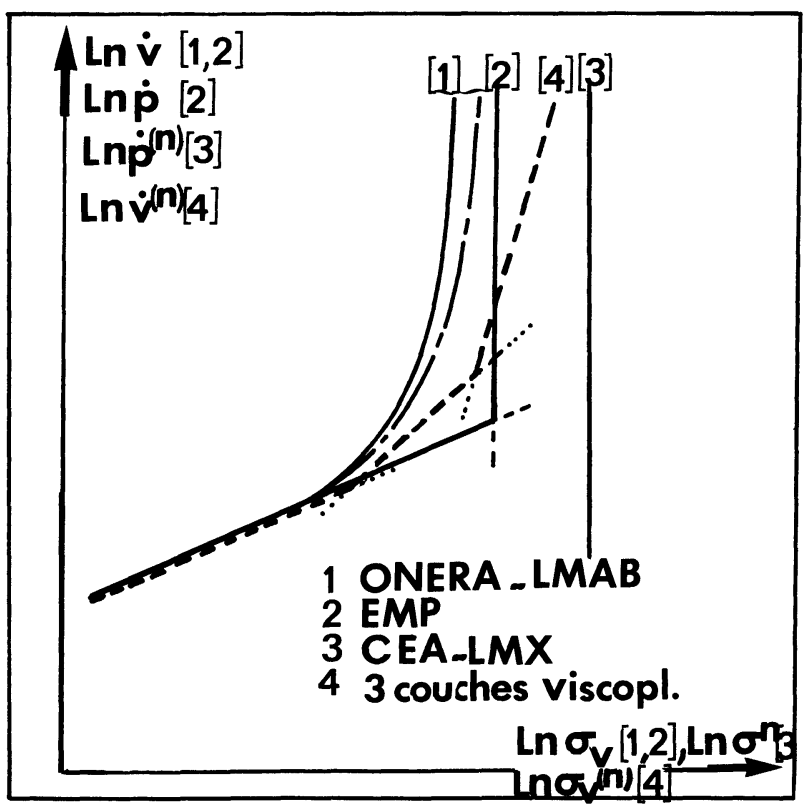

Fig. 19. - Description schématique du traitement de la non-linéarité de l'équation d'état, dans les différentes approches.

[Schematic description of the non-linearity of the state equation, in the various approaches.]

5.2.3.1 Le modèle ONERA-LMT Cachan. - La non-linéarité est traitée continuement (modèle unifié) par l'adjonction à la loi puissance d'un terme multiplicatif exponentiel, soit d'après les équations (17) et (53b) :

$$
\begin{aligned}
& \dot{\varepsilon}_{i j}^{\mathrm{v}}=\frac{3}{2}\left\langle\frac{\sigma_{\mathrm{v}}}{K\left(R^{\mathrm{v}}\right)}\right\rangle^{n^{*}} \exp \alpha_{0}\left\langle\frac{\sigma_{\mathrm{v}}}{K\left(R^{\mathrm{v}}\right)}\right\rangle^{n^{*}+1} \times \\
& \times \frac{\sigma_{i j}^{\prime}-X_{i j}^{\mathrm{v}^{\prime}}}{J_{2}\left(\underline{\mathbf{\sigma}}-\underline{\mathbf{X}}^{\mathrm{v}}\right)}
\end{aligned}
$$

et pour le module $\dot{v}=\sqrt{\frac{2}{3} \dot{\varepsilon}_{i j}^{\mathrm{v}} \dot{\varepsilon}_{i j}^{\mathrm{v}}}$ :

$$
\dot{v}=\left\langle\frac{\sigma_{\mathrm{v}}}{K\left(R^{\mathrm{v}}\right)}\right\rangle^{n^{*}} \exp \alpha_{0}\left\langle\frac{\sigma_{\mathrm{v}}}{K\left(R^{\mathrm{v}}\right)}\right\rangle^{n^{*}+1} .
$$

D'après $(53 b)$,

$$
\sigma_{\mathrm{v}}=J_{2}\left(\underline{\mathbf{g}}-\underline{\mathbf{X}}^{\mathrm{v}}\right)-R^{\mathrm{v}}-R_{0}^{\mathrm{v}}
$$

En effet, on se souvient que les termes supplémentaires, en $J_{2}^{2}\left(\underline{\mathbf{X}}^{\mathrm{v}}\right)$ et $J_{2}^{2}\left(\underline{\boldsymbol{\alpha}}^{\mathrm{v}}\right)$ de l'équation (32), pour introduire l'écrouissage cinématique non linéaire se compensent exactement, d'où l'écriture de $\sigma_{\mathrm{v}}$. La relation (66b) est représentée schématiquement par la courbe (1) sur la figure 19 et correspond sensiblement à définir un exposant $N$ variable avec $\sigma_{\mathrm{v}}$ et tel que :

$$
\begin{aligned}
N & =\left(\frac{\partial \operatorname{Ln} \dot{v}}{\partial \operatorname{Ln} \sigma_{\mathrm{v}}}\right)_{T} \\
& =n^{*}+\alpha_{0}\left(n^{*}+1\right)\left(\frac{\sigma_{\mathrm{v}}}{K\left(R^{v}\right)}\right)^{n^{*}+1}
\end{aligned}
$$

Ainsi, $N$ croît avec $\sigma_{v}$ de $N=n *$ à $N=\infty$. Dans les équations (66a et $\mathrm{b}$ ), $n^{*}$ est une constante indépendante de la température et $K$ une fonction de la variable isotrope $R^{v}$, soit :

$$
K\left(R^{v}\right)=K_{0}+\alpha_{\mathrm{K}} R^{\mathrm{v}} \quad \text { avec } \quad R(0)=0 .
$$

L'introduction de (68) permet de rendre compte de l'accroissement de $\sigma_{v}$ avec la déformation $v$. En effet, si l'on prend l'équation (66b) sous la forme d'une loi puissance, son inversion conduit à $\sigma_{\mathrm{v}}=$ $K(\dot{v})^{1 / n^{*}}$, c'est-à-dire qu'à $\dot{v}$ fixée, $\sigma_{\mathrm{v}}$ l'est également avec $K$ constant, ce qui est contraire à l'expérience (Fig. 18). Le choix de $K$ fonction de $R^{v}$ plutôt que de la déformation cumulée $v$ provient de ce que durant un écoulement stationnaire $v$ continue à augmenter alors que $R^{\mathrm{v}}$ est constant, ce qui est conforme aux observations expérimentales. Par exemple, sur la figure 18, courbes (7) et (8), aux plus hautes températures, lorsque le matériau s'écoule sous contrainte constante pour une vitesse imposée, on montre que $\sigma_{v}$ est indépendant de la déformation. En conclusion, ce modèle fait intervenir la déformation cumulée par le biais de $R^{v}$, sous une forme mixte, c'est-àdire additive (dans $\sigma_{v}$ ) et multiplicative (dans $K\left(R^{v}\right)$ ). Cependant, l'équation (66b) n'est pas inver- 
sable, c'est-à-dire que l'on ne peut pas exprimer simplement $\sigma_{\mathrm{v}}=F^{-1}(\dot{v})$, ce qui peut poser quelques problèmes au niveau de l'identification du modèle. Par contre, la vitesse $\dot{\varepsilon}_{i j}^{v}$ (Eq. (66a)) dérive bien analytiquement d'un potentiel $\Omega$, tel que $\Omega_{\mathrm{v}}$ (Eq. (32)) s'écrit :

$$
\Omega_{\mathrm{v}}=\frac{K\left(R^{\mathrm{v}}\right)}{\alpha_{0}\left(n^{*}+1\right)} \exp \alpha_{0}\left\langle\frac{\sigma_{\mathrm{v}}}{K\left(R^{\mathrm{v}}\right)}\right\rangle^{n^{*}+1} .
$$

L'identification complète de $\Omega$ (intervention de $\Omega_{\mathrm{r}}$ ) sera mentionnée ultérieurement.

5.2.3.2 Le modèle LMAB. - A l'instar du modèle ONERA, celui du LMAB est unifié et traite continuement la relation $\operatorname{Ln} \dot{v}=f\left(\operatorname{Ln} \sigma_{\mathrm{v}}\right)$ (courbe (1), Fig. 19) par l'introduction d'une fonction hyperbolique. A l'aide des équations (18) et (54a), on arrive à :

$$
\begin{aligned}
\dot{\varepsilon}_{i j}^{\mathrm{v}}= & \frac{3}{2} \dot{v}_{0}\left(\frac{K\left(R^{\mathrm{v}}\right)+\sigma_{0}^{*}}{\sigma_{0}^{*}}\right)^{n^{*}} \times \\
& \times \sinh \left(\frac{\sigma_{\mathrm{v}}}{K\left(R^{\mathrm{v}}\right)+\sigma_{0}^{*}}\right)^{n^{*}} \cdot \frac{\sigma_{i j}^{\prime}-X_{i j}^{\mathrm{v}^{\prime}}}{J_{2}\left(\underline{\mathbf{\sigma}}-\underline{\mathbf{X}}^{\mathrm{v}}\right)} .
\end{aligned}
$$

$\sigma_{0}^{*}$ est une constante et comme $K\left(R^{\mathrm{v}}\right) \gg \sigma_{0}^{*}$, la relation (70) se simplifie sous la forme :

$$
\begin{aligned}
\dot{\varepsilon}_{i j}^{\mathrm{v}}=\frac{3}{2} \dot{v}_{0}( & \left.\frac{K\left(R^{\mathrm{v}}\right)}{\sigma_{0}^{*}}\right)^{n^{*}} \times \\
& \times \sinh \left(\frac{\sigma_{\mathrm{v}}}{K\left(R^{v}\right)}\right)^{n^{*}} \frac{\sigma_{i j}^{\prime}-X_{i j}^{\mathrm{v}^{\prime}}}{J_{2}\left(\underline{\mathbf{g}}-\underline{\mathbf{X}}^{\mathrm{v}}\right)},
\end{aligned}
$$

et pour le module de la vitesse :

$$
\dot{v}=\dot{v}_{0}\left(\frac{K\left(R^{v}\right)}{\sigma_{0}^{*}}\right)^{n^{*}} \sinh \left(\frac{\sigma_{\mathrm{v}}}{K\left(R^{\mathrm{v}}\right)}\right)^{n^{*}} \text {. }
$$

D'après (54a), on a :

$$
\sigma_{\mathrm{v}}=J_{2}\left(\underline{\mathbf{\sigma}}-\underline{\mathbf{X}}^{\mathrm{v}}\right),
$$

puisque les termes en $J_{2}^{2}\left(\underline{\underline{X}}^{\mathbf{v}}\right)$ et $J_{2}^{2}\left(\underline{\alpha}^{\mathbf{v}}\right)$ dans le potentiel $\Omega$ (Eq. (47)) s'annulent. L'exposant $N$ défini à partir de l'équation (71b) s'écrit :

$$
\begin{aligned}
N & =\left(\frac{\partial \operatorname{Ln} \dot{v}}{\partial \operatorname{Ln} \sigma_{\mathrm{v}}}\right)_{\mathrm{T}} \\
& =n^{*}\left(\frac{\sigma_{\mathrm{v}}}{K\left(R^{v}\right)}\right)^{n^{*}} \operatorname{coth}\left(\frac{\sigma_{\mathrm{v}}}{K\left(R^{v}\right)}\right)^{n^{*}}
\end{aligned}
$$

et, comme pour le modèle ONERA (Eq. (67)), croît avec $\sigma_{\mathrm{v}}$ de $N=n^{*}$ pour $\left(\frac{\sigma_{\mathrm{v}}}{K\left(R^{v}\right)}\right)^{n^{*}}$ faible à $N=\infty$ pour les fortes valeurs de l'argument.

Les relations (71a et b) suscitent plusieurs commentaires. Cette loi a été directement identifiée à partir de mesures expérimentales telles que celles reproduites sur la figure 17, et pour une gamme de températures variant de 525 à $750^{\circ} \mathrm{C}$. Ainsi, on peut montrer que $\dot{v}_{0}$ et $n^{*}$ sont fonctions de la température [7,60], soit :

$$
\begin{aligned}
\dot{v}_{0} & =C^{*} \frac{D_{\mathrm{Fe}} G b}{k T} \text { pour } \sigma_{0}^{*}=0,15 \mathrm{MPa} \\
n^{*} & =\frac{3500}{T}-1,8 .
\end{aligned}
$$

$D_{\mathrm{Fc}}$ est le coefficient de diffusion en volume du fer au sein de la solution solide austénitique, $G$ le module de cisaillement, $k$ la constante de Boltzman et $b$ le vecteur de Burgers. Les relations (71b) et (73) dérivent de l'analyse thermodynamique du glissement (ou de la montée) thermiquement activé des dislocations à partir de la relation d'Orowan, et à l'aide du formalisme d'Eyring, on aboutit naturellement à une forme en sinus hyperbolique. On peut noter que l'extension de l'équation (74) aux plus faibles températures $\left(T \leqq 500^{\circ} \mathrm{C}\right)$ fait intervenir un coefficient de diffusion effectif qui tient compte des diffusions en volume et sur les courts-circuits de diffusion. Pour les mêmes raisons explicitées précédemment, $K$ est une fonction de $R^{\mathrm{v}}$ (Fig. 18), soit :

$$
K=\alpha_{K}(T) R^{\mathrm{v}} \text { avec } R^{\mathrm{v}}(0)=R_{0}^{\mathrm{v}} .
$$

Pour les plus faibles valeurs de son argument, la relation (71b) est identique à la loi puissance classique, répertoriée pour de nombreux alliages,

$$
\dot{v}=\dot{v}_{0}\left(\frac{\sigma_{\mathrm{v}}}{\sigma_{0}^{*}}\right)^{n^{*}}
$$

Dans ce cas, il existe une relation biunivoque entre $\dot{v}$ et $\sigma_{v}$, ce qui n'est plus vrai lorsque $\sigma_{\mathrm{v}}$ augmente (contribution du terme $K\left(R^{v}\right)$ ). Ce type de comportement, équations $(71 \mathrm{a}, \mathrm{b}),(74)$ et (75), ajusté sur les points expérimentaux est illustré sur la figure 20 , où l'on montre, en coordonnées logarithmiques et pour l'ensemble des températures étudiées, la corrélation $\frac{\dot{v} k T}{D_{\mathrm{Fe}} G b}=f\left(\frac{\sigma_{\mathrm{v}}}{\sigma_{0}^{*}}\right)^{n^{*}(T)}$. Les quatre courbes tracées correspondent à quatre valeurs de $K\left(R^{v}\right)$. Un autre avantage de la loi (71b) est qu'elle est analytiquement inversable, ce qui permet d'exprimer $\sigma_{\mathrm{v}}$ sous la forme :

$$
\begin{aligned}
\sigma_{\mathrm{v}}= & \alpha_{k}(T) R^{\mathrm{v}} \times \\
& \times\left(\operatorname{Argsinh}\left(\frac{\dot{v}}{\dot{v}_{0}}\left(\frac{\sigma_{0}^{*}}{\alpha_{k}(T) R^{\mathrm{v}}}\right)^{n^{*}(T)}\right)\right)^{1 / n^{*}(T)}
\end{aligned}
$$

ce qui simplifie la tâche d'identification du modèle et singulièrement celle des courbes monotones à vitesse imposée qui, selon (58) et (60b), s'écrivent $\sigma=$ 


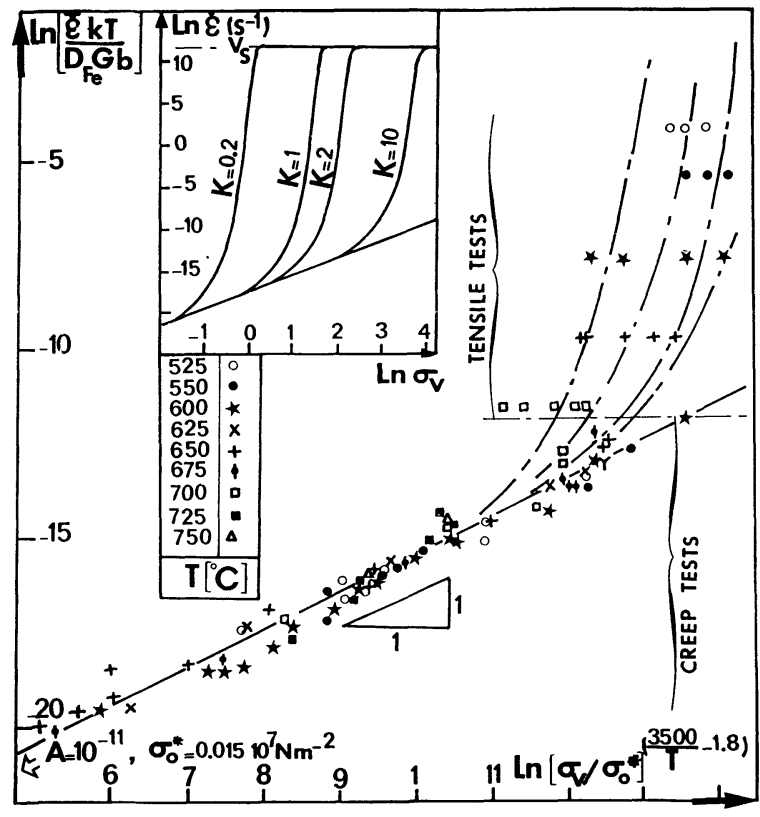

Fig. 20. - Vérification de l'équation (71b), identifiée sur les résultats expérimentaux obtenus pour différentes températures. (D'après Delobelle et Oytana [60], avec l'amabilité de $\mathrm{Nucl}$. Eng. Des.).

[Verification of equation (71b) as identified from the experimental results obtained at various temperatures. (From Delobelle and Oytana [60], with courtesy of $\mathrm{Nucl}$. Eng. Des.).]

$\sigma_{\mathrm{i}}+\sigma_{\mathrm{v}}$, avec $\sigma_{\mathrm{v}}$ directement accessible lorsque l'on connaît $\dot{v}$ et $R^{\mathrm{v}}$.

Par contre, l'équation (71b) ne dérive pas directement d'un potentiel $\Omega_{\mathrm{v}}$ exprimé à partir de fonctions simples connues ( $n *$ est continuement variable avec la température) mais prend la forme d'un développement de Taylor, qui s'annule à l'origine et dont la convexité est assurée. Une autre possibilité qui conduit à une écriture très voisine de la relation (71b) est de prendre $\Omega_{\mathrm{v}}$ sous la forme :

$\Omega_{\mathrm{v}}=2 \frac{\dot{v}_{0}}{\sigma_{0}^{* n}} \frac{\left(K\left(R^{\mathrm{v}}\right)\right)^{n^{*}+1}}{n^{*}+1} \cosh \left(\frac{\sigma_{\mathrm{v}}}{K\left(R^{v}\right)}\right)^{\left(n^{*}+1\right) / 2}$,

d'où l'on déduit :

$$
\begin{aligned}
\dot{v}=\dot{v}_{0}\left(\frac{K\left(R^{v}\right)}{\sigma_{0}^{*}}\right)^{n^{*}} & \left(\frac{\sigma_{\mathrm{v}}}{K\left(R^{v}\right)}\right)^{\left(n^{*}-1\right) / 2} \times \\
& \times \sinh \left(\frac{\sigma_{\mathrm{v}}}{K\left(R^{v}\right)}\right)^{\left(n^{*}+1\right) / 2} .
\end{aligned}
$$

$\mathrm{Au}$ terme puissance près, (79) est identique à (71b) et se développe, pour les faibles valeurs de l'argument, selon la loi puissance (76). De plus, compte tenu que $n^{*}$ est faible ( $n^{*}$ voisin de 2 (Eq. (74))), l'exposant intervenant dans le sinus hyperbolique est nettement supérieur à celui du terme puissance, d'où l'extrême proximité des représentations graphiques des relations (71b) et (79). La commodité de la forme (71b) par rapport à (79) qui n'est pas inversable, quitte à avoir un potentiel $\Omega_{\mathrm{v}}$ sous la forme d'un développement, nous semble préférable, tout en sachant que l'équation (79) conduit à des résultats comparables.

5.2.3.3 Remarques sur ces deux modèles unifiés, comparaison avec d'autres modèles et particulièrement les théories isotropes de l'écrouissage par la déformation. - On remarque une certaine cohérence entre les équations (66b) et (71b) avec les modèles de Miller [46, 61] et l'analyse de Ohno et coll. [62] qui s'écrivent respectivement, avec les mêmes notations :

$$
\dot{v}=\dot{v}_{0}(T)\left(\sinh \left(\frac{\sigma_{\mathrm{v}}}{R^{\mathrm{v}}}\right)^{1,5}\right)^{n^{*}}
$$

$\operatorname{avec} \sigma_{\mathrm{v}}=J_{2}\left(\underline{\mathbf{G}}-\underline{\mathbf{X}}^{\mathrm{v}}\right)$,

$$
\text { et } \quad \dot{v}=\dot{v}_{0}(T)\left(\exp -\frac{R^{v}}{K}\right) \cdot\left(\sinh \left(\frac{\sigma_{\mathrm{v}}}{K}\right)\right)^{n^{*}}
$$

avec $\sigma_{\mathrm{v}}=J_{2}\left(\underline{\mathbf{g}}-\underline{\mathbf{X}}^{\mathrm{v}}\right)$ et $K$ constant .

Cependant, on enregistre un certain avantage, du point de vue réalisme au détriment de l'identification, pour les deux modèles unifiés présentés qui font intervenir la déformation plastique cumulée (variable $R^{\text {y }}$ ) sous une forme mixte, additive et multiplicative, par rapport aux équations (80) et (81) où elle n'intervient que sous une forme multiplicative.

A ce stade, il semble nécessaire de montrer que ces modèles «évolués » aboutissent, moyennant de nombreuses simplifications, aux théories simples, isotropes, de l'écrouissage par la déformation, très souvent utilisées dans les codes de calcul mais dont la véracité dans le cas des chargements non monotones laisse grandement à désirer. En effet, en faisant abstraction de l'écrouissage cinématique, on peut écrire simplement $\dot{v}$ sous la forme d'une loi puissance :

$$
\dot{v}=\dot{v}_{0}\left\langle\frac{\sigma_{\mathrm{v}}}{K\left(R^{v}\right)}\right\rangle^{n^{*}} \text { avec } \sigma_{\mathrm{v}}=J_{2}(\underline{\mathbf{g}})-R^{\mathrm{v}}
$$

et

$$
K\left(R^{v}\right)=\alpha_{K} R^{v},
$$

qui correspond aux équations (66b) avec $\alpha_{0}=0$, (71b) avec $\sigma_{0}^{*}=K\left(R^{v}\right)$ dans le cas des faibles valeurs de l'argument, et aux relations (68) et (75). De plus, si l'on écrit $R^{v}$ selon une loi puissance, soit d'après les équations (14) et (25),

$$
R^{\mathrm{v}}=\rho \frac{\partial \psi}{\partial r^{v}}=b v^{1 / m^{*}},
$$

on aboutit aisément à :

$$
\dot{v}=\dot{v}_{0}\left\langle\frac{J_{2}(\underline{\mathbf{g}})-b v^{1 / m^{*}}}{\alpha_{K} b v^{1 / m^{*}}}\right\rangle^{n^{*}} .
$$


Cette relation constitue le modèle de Lemaître [63] et si maintenant on considère que $b$ est petit, on arrive à la loi bien connue de Norton-Hoff :

$$
\dot{v}=\dot{v}_{0}\left(\alpha_{K} b\right)^{-n^{*}}\left\{J_{2}(\underline{\mathbf{\sigma}})\right\}^{n^{*}} v^{-n^{*} / m^{*}},
$$

soit sous sa forme unidimensionnelle classique,

$$
\sigma=c \dot{\varepsilon}^{n^{\prime}} \varepsilon^{m^{\prime}},
$$

avec $n^{\prime}=\frac{1}{n^{*}}, m^{\prime}=\frac{1}{m^{*}}$ et $c=\alpha b / \dot{v}_{0}^{1 / n^{*}}$. Pour $n^{\prime}=0$, cas de la plasticité indépendante du temps, on retrouve la loi bien connue de Ludwik-Hollomon, $\sigma=c \varepsilon^{m^{\prime}}$, et à l'opposé, pour $m^{\prime}=0$, cas de la viscosité, la loi de Norton $\sigma=c \dot{\varepsilon}^{n^{\prime}}$. En suivant une démarche similaire, on peut également montrer [64], qu'à notre connaissance, toutes les lois semi-empiriques, plus ou moins sophistiquées, proposées jusqu'à nos jours pour traiter les courbes de traction monotone à vitesse imposée (Ludwik-Hollomon [65], Voce [66], Ludwigson [67], Ghosh [68], Piatti [69]) dérivent directement du formalisme unifié proposé, à savoir : une équation d'état plus ou moins fortement non linéaire (par exemple les équations (66b), (71b), (82)), une intervention de la déformation cumulée sous des formes additive, multiplicative ou mixte, une cinétique d'évolution pour $R^{\mathbf{v}}$ (par exemple (83)). La forme finale ne dépend que du choix des fonctionnelles à chacune des étapes qui viennent d'être énumérées.

Les lacunes de ces modèles isotropes sont bien connues (particulièrement dans le cas de retour en arrière, de l'effet Bauschinger, ...) et une amélioration astucieuse et conséquente de la formulation (85a), due à Murakami et Ohno [70], permettant de lever partiellement ces difficultés et sur laquelle nous reviendrons ultérieurement, consiste à définir une surface de recouvrance de l'écrouissage dans l'espace des déformations visqueuses.

5.2.3.4 Le modèle partitionné de l'EMP. - Le modèle partitionné de l'EMP consiste à traiter la non-linéarité de la représentation de la figure 19 en adoptant deux valeurs discrètes de l'exposant $N$ (courbe (2), Fig. 19), soit : $N=\left(\frac{\partial \operatorname{Ln} \dot{p}}{\partial \operatorname{Ln} \sigma_{\mathrm{v}}}\right)_{\mathrm{T}}=\infty$ (cas de la plasticité indépendante du temps) et $N=\left(\frac{\partial \operatorname{Ln} \dot{v}}{\partial \operatorname{Ln} \sigma_{\mathrm{v}}}\right)_{\mathrm{T}}=n^{*}$ (cas de la viscosité pure). On rejoint les deux cas cités précédemment de la loi de Norton-Hoff, avec respectivement, $n^{\prime}=0$ et $m^{\prime}=0$. Cette représentation est assez conforme à la réalité, sauf au niveau du raccordement brusque des demi-droites. La déformation totale est la somme des deux composantes : visqueuse et plastique (Eq. (55d)). Pour la déformation visqueuse, on a simplement la loi de Norton :

$$
\dot{\varepsilon}_{i j}^{v}=\frac{3}{2}\left\langle\frac{\sigma_{v}}{K}\right\rangle^{n^{*}} \frac{\sigma_{i j}^{\prime}-X_{i j}^{\prime}}{J_{2}\left(\underline{\mathbf{\sigma}}-\underline{\mathbf{X}}^{\mathrm{v}}\right)},
$$

et en module :

$$
\dot{v}=\left(\frac{\sigma_{\mathrm{v}}}{K}\right)^{n^{*}}
$$

avec $\sigma^{\mathrm{v}}=J_{2}\left(\underline{\mathbf{g}}-\underline{\mathbf{X}}^{\mathrm{v}}\right)-R^{\mathrm{v}}-R_{0}^{\mathrm{v}}\left(\right.$ Eq. (55c)), $n^{*}$ et $K$ sont des constantes. $\dot{v}$ dérive très aisément d'un potentiel $\Omega_{\mathrm{v}}$ tel que :

$$
\Omega_{\mathrm{v}}=\frac{K}{n^{*}+1}\left\langle\frac{\sigma_{\mathrm{v}}}{K}\right\rangle^{n^{*}+1} .
$$

Le fait que $K$ soit une constante est en accord avec le modèle LMAB pour les plus faibles valeurs de l'argument $\left(\frac{\sigma_{\mathrm{v}}}{K}\right)$ des équations (71), d'où la relation : $K=\sigma_{0}^{*} \dot{v}_{0}^{-1 / n^{*}}$ (Eqs. (86b) et (76)). De plus, l'équation de consistance $f=\dot{f}=0$ fixant $\underline{\mathbf{g}}$ sur la surface plastique $f$, déplace le point de raccordement des deux demi-droites $\left(\dot{v}, \dot{p}\right.$ fonction de $\left.\sigma_{v}\right)$, ce qui est tout à fait conforme aux observations expérimentales de la figure 17 (courbe (1)). Dans le modèle LMAB, ce point avait été intégré en prenant $K$ fonction de $R^{v}$ dans l'équation (71). En ce qui concerne la surface de plasticité, elle s'écrit simplement $f=J_{2}\left(\underline{\mathbf{\sigma}}-\underline{\mathbf{X}}^{\mathrm{p}}\right)-R^{\mathrm{p}}-R_{0}^{\mathrm{p}}$ (voir Eq. (55a)) et d'après la relation (23), la déformation plastique cumulée est donnée par le multiplicateur plastique, $\dot{\lambda}=\dot{p}$. Compte tenu des équations cinétiques de $X^{p}$ (Eq. (40a)) et $R^{p}$, que nous expliciterons en détail dans le paragraphe suivant, on arrive, tous calculs effectués, à :

$$
\dot{p}=\left\langle\frac{\underline{\dot{\mathbf{\sigma}}} \underline{\underline{m}}_{\mathrm{p}}-K^{\mathrm{vp}}\left(\underline{\dot{\varepsilon}}^{\mathrm{v}} \underline{\underline{m}}_{\mathrm{p}}-C^{\mathrm{v}} \dot{v} \underline{\underline{\mathbf{X}}}^{\mathrm{v}} \underline{\underline{m}}_{\mathrm{p}}\right)}{b^{\mathrm{p}}\left(Q^{\mathrm{p}}-R^{\mathrm{p}}\right)+K^{\mathrm{p}}\left(1-C^{\mathrm{p}} \underline{\underline{\mathbf{x}}}^{\mathrm{p}} \underline{\underline{m}}_{\mathrm{p}}\right)}\right\rangle,
$$

avec $\underline{\underline{m}}_{\mathrm{p}}=\sqrt{\frac{3}{2}} \frac{\sigma_{i j}^{\prime}-X_{i j}^{\mathrm{p}^{\prime}}}{J_{2}\left(\underline{\mathbf{\sigma}}-\underline{\underline{\mathbf{X}}}^{\mathrm{p}}\right)}$. Le module de la déformation totale s'écrit donc : $\bar{\varepsilon}=\dot{v}+\dot{p}$ (Eqs. (86) et (88)).

Remarque: Dans une version initiale du modèle [32], les auteurs ne compensent pas les termes quadratiques en $J_{2}^{2}(\underline{\mathbf{X}})$ dans les potentiels $f$ et $\Omega$ (introduit pour décrire l'écrouissage cinématique non linéaire) par les termes en $J_{2}^{2}(\underline{\alpha})$ (Eq. (37)). Dans ce cas, $\dot{v}$ et $\dot{p}$ s'expriment respectivement par :

$$
\dot{v}=\left\langle\frac{\sigma_{\mathrm{v}}}{K}\right\rangle^{n^{*}}, \quad \text { mais avec } \quad \sigma_{\mathrm{v}}=J_{2}\left(\underline{\mathbf{g}}-\underline{\mathbf{X}}^{\mathrm{v}}\right)+\frac{1}{2} C^{\mathrm{v}} J_{2}^{2}\left(\underline{\mathbf{X}}^{\mathrm{v}}\right)-R^{\mathrm{v}}-R_{0}^{\mathrm{v}}
$$


et

$$
\dot{p}=\left\langle\frac{\underline{\dot{\sigma}}_{\underline{\mathrm{n}}_{\mathrm{p}}}-K^{\mathrm{vp}}\left(\underline{\dot{\varepsilon}}^{\mathrm{v}} \underline{\underline{n}}_{\mathrm{p}}-C^{\mathrm{v}} \dot{v} \underline{\mathbf{X}}^{\mathrm{v}} \underline{\underline{n}}_{\mathrm{p}}\right)+C^{\mathrm{p}} K^{\mathrm{vp}}\left(\underline{\mathbf{X}}^{\mathrm{p}} \underline{\dot{\varepsilon}}^{\mathrm{v}}-C^{\mathrm{v}} \dot{v} \underline{\underline{X}}^{\mathrm{v}} \underline{\mathbf{X}}^{\mathrm{p}}\right)}{b^{\mathrm{p}}\left(Q_{1}^{\mathrm{p}}-R^{\mathrm{p}}\right)+K^{\mathrm{p}}\left(1-C^{\mathrm{p}} \underline{\mathbf{X}}^{\mathrm{p}} \underline{\underline{m}}_{\mathrm{p}}\right)-C^{\mathrm{p}} K^{\mathrm{p}} \underline{\underline{X}}^{\mathrm{p}}\left(\underline{\mathrm{n}}_{\mathrm{p}}-C^{\mathrm{p}} \underline{\underline{X}}^{\mathrm{p}}\right)}\right\rangle .
$$

Les formulations (88) et (89) diffèrent essentiellement, lors des chargements multiaxés hors phase.

On peut noter que Ohashi et al. [71] ont montré clairement la nécessité d'avoir un couplage dans les approches partitionnées. Pour leur part, ils écrivent $\dot{\varepsilon}_{i j}^{\mathrm{T}}=\dot{\varepsilon}_{i j}^{\mathrm{v}}+\dot{\varepsilon}_{i j}^{\mathrm{p}}$; la vitesse de déformation visqueuse $\dot{\varepsilon}_{i j}^{\mathrm{v}}$ est donnée simplement par une loi du type Norton-Hoff (Eq. (85a)) et $\dot{\varepsilon}_{i j}^{\mathrm{p}}$ dérive d'une surface de charge $f$ à écrouissage cinématique. Le couplage s'effectue sur cette variable, d'une manière différente de celui du modèle de l'EMP, à savoir, avec nos notations :

$\dot{X}_{i j}^{\mathrm{p}}=K\left[\left(\dot{\varepsilon}_{i j}^{\mathrm{p}}+\beta^{+} \dot{\varepsilon}_{i j}^{\mathrm{v}}\right)-C X_{i j}^{\mathrm{p}}\left|\dot{\varepsilon}_{i j}^{\mathrm{p}}+\beta^{+} \dot{\varepsilon}_{i j}^{\mathrm{v}}\right|\right]$.

Le couplage s'effectue par le paramètre $\beta^{+}$et pour un chargement radial monotone, on identifie les deux modèles avec $K^{\mathrm{p}}=K, K^{\mathrm{vp}}=K \beta^{+}$et $C=$ $C^{\mathrm{p}}=C^{\mathrm{v}}$. Bien évidemment pour des trajets non radiaux, ces deux modèles sont différents.

5.2.3.5 Les modèles multicouches. - Les modèles multicouches présentés et utilisés au CEA et au LMX sont écrits dans des versions purement plastiques (avec effet du temps lié à la restauration dans le modèle CEA), c'est-à-dire que chaque couche $(n)$ possède un potentiel plastique $f^{(n)}$ qui devient actif lorsque la contrainte (locale ou globale) atteint cette surface. L'exposant de viscosité $N$ est donc infini pour chacune des couches (courbe (3) de la Fig. 19).

Cependant, compte tenu des différentes manifestations expérimentales de la viscosité (Fig. 18 par exemple), même en l'absence de restauration (cas du fluage de l'acier 17-12 SPH au voisinage de l'ambiante où les phénomènes thermiquement activés sont absents), il semble indispensable de posséder une version viscoplastique de ces modèles. En fait, cette extension est théoriquement aisée et consiste à écrire pour chaque sous-couche $(n)$ un potentiel de dissipation $\Omega_{\mathrm{v}}^{(n)}$ dont la vitesse $\dot{\varepsilon}_{i j}^{(n)}$ en dérive, soit pour le modèle CEA :

et

$$
\begin{aligned}
\dot{\varepsilon}_{i j}^{\mathrm{v}} & =\frac{\partial \Omega_{\mathrm{v}}^{(n)}}{\partial \sigma_{i j}^{(n)}}, \\
\dot{\varepsilon}_{i j}^{\mathrm{v}(n)} & =\frac{\partial \Omega_{\mathrm{v}}^{(n)}}{\partial \sigma_{i j}},
\end{aligned}
$$

pour le modèle LMX.

En calquant notre démarche sur celle des modèles précédents, pour chaque couche, on écrira :

$$
\dot{v}^{(n)}=\sqrt{\frac{2}{3} \dot{\varepsilon}_{i j}^{\mathrm{v}(n)} \dot{\varepsilon}_{i j}^{\mathrm{v}(n)}}=\left\langle\frac{\sigma_{\mathrm{v}}^{(n)}}{K R_{0}^{\mathrm{v}(n)}}\right\rangle^{n^{*}(n)},
$$

avec $\quad \sigma_{\mathrm{v}}^{(n)}=J_{2}\left(\underline{\mathbf{\sigma}}^{(n)}-\underline{\mathbf{X}}^{\mathrm{v}(n)}\right)-R_{0}^{\mathrm{v}(n)}$,

pour le modèle parallèle, et $\dot{v}$ identique à l'équation (93a) mais avec :

$$
\sigma_{\mathrm{v}}^{(n)}=J_{2}\left(\underline{\mathbf{\sigma}}-\underline{\mathbf{X}}^{\mathrm{v}(n)}\right)-R_{0}^{\mathrm{v}(n)},
$$

pour le modèle série. L'écriture des potentiels $\Omega_{\mathrm{v}}^{(n)}$ est immédiate (Eq. (87)).

La non-linéarité de la représentation de la figure $19, \dot{v}=f\left(\sigma_{v}\right)$. sera donc restituée par une courbe brisée, dont chaque branche aura pour exposant $n^{*(n)}$ (courbe (4), Fig. 19, avec trois couches par exemple). Plus le nombre de couches sera élevé, avec une distribution large de $n^{*(n)}$, plus la caractéristique globale sera proche de celle de la représentation unifiée.

Une autre possibilité émise par Blanchard [72] est de considérer un modèle multicouche unifié (modèle du type CEA) dont l'équation d'état par couche est très proche de celle du modèle LMAB. En effet, il propose d'écrire :

$$
\dot{v}^{(n)}=\dot{v}_{0} \sinh \left(\frac{\sigma_{\mathrm{v}}^{(n)}}{R_{0}^{\mathrm{v}(n)}}\right)^{n^{*}},
$$

avec l'exposant $n *$ constant et :

$$
\sigma_{\mathrm{v}}^{(n)}=J_{2}\left(\underline{\mathbf{\sigma}}^{(n)}-\underline{\mathbf{X}}^{\mathrm{v}(n)}\right),
$$

ce qui s'apparente à l'équation (71b). L'expression (95a) est inversable et permet d'exprimer $\sigma_{\mathrm{v}}^{(n)}$ en fonction de $\dot{v}^{(n)}$, soit pour une couche :

$$
\sigma_{\mathrm{v}}^{(n)}=R_{0}^{\mathrm{v}(n)}\left[\operatorname{Argsinh}\left(\frac{\dot{v}^{(n)}}{\dot{v}_{0}}\right)\right]^{1 / n^{*}},
$$

très proche de (77). Les variations de la composante visqueuse avec la déformation (Fig. 18) sont donc prises en compte par la distribution des rayons $R_{0}^{\mathrm{v}(n)}$ des surfaces de charge imposant l'état de chacune des couches (élastique ou viscoplastique selon les équations (56c et d)).

\subsection{LES CINÉTIQUES D'ÉVOLUTION DES VARIABLES INTERNES.}

5.3.1 Le modèle ONERA-LMT Cachan. - Conformément aux équations (53d), pour ce modèle, il s'agit d'identifier les cinétiques de $X_{i j}^{\mathrm{v}}$ et $R^{\mathrm{v}}$. Dans le paragraphe 4 , nous avons montré comment le choix du potentiel $\Omega$ (Eq. (32)) permet d'aboutir à la stricte définition de l'écrouissage cinématique non linéaire avec restauration (Eq. (35)). Aux tempéra- 
tures intermédiaires $\left(T / T_{\mathrm{F}} \geqq 0,3\right)$ les effets de restauration intrinsèquement liés au temps ont été clairement mis en évidence (par exemple Fig. 10 et 11) et à l'aide de séquences expérimentales bien précises de traction-compression ou torsion alternée avec temps de maintien à contrainte nulle [79] ou au maximum d'un cycle [17], il est possible de séparer les contributions à la restauration des variables cinématique et isotrope. On montre ainsi que généralement, ces deux variables possèdent une composante de restauration.

D'un point de vue pratique, de façon à mieux ajuster la non-linéarité des courbes monotones et cycliques à vitesse imposée, les auteurs suggèrent l'écriture :

$$
X_{i j}^{\mathrm{v}}=\sum_{n} X_{i j}^{\mathrm{v}(n)}
$$

$[8,17]$ et considèrent que $n=2$ est suffisant. On écrira donc :

$$
X_{i j}^{\mathrm{v}}=X_{i j}^{\mathrm{v}(1)}+X_{i j}^{\mathrm{v}(2)}
$$

avec :

$$
\begin{aligned}
\dot{X}_{i j}^{\mathrm{v}(1)}= & \frac{2}{3} a^{(1)} \dot{\varepsilon}_{i j}^{\mathrm{v}}-C(v) X_{i j}^{\mathrm{v}(1)} \dot{v}- \\
& -\beta J_{2}\left(\underline{\mathbf{X}}^{\mathrm{v}}\right)^{r} \frac{X_{i j}^{\mathrm{v}(1)}}{J_{2}\left(\underline{\mathbf{X}}^{\mathrm{v}}\right)} \\
\dot{X}_{i j}^{\mathrm{v}(2)}= & \frac{2}{3} a^{(2)} \dot{\varepsilon}_{i j}^{\mathrm{v}}-C(v) X_{i j}^{\mathrm{v}(2)} \dot{v}- \\
& -\beta J_{2}\left(\underline{\mathbf{X}}^{\mathrm{v}}\right)^{r} \frac{X_{i j}^{\mathrm{v}(2)}}{J_{2}\left(\underline{\mathbf{X}}^{\mathrm{v}}\right)}
\end{aligned}
$$

avec les conditions initiales $X_{i j}^{\mathrm{v}(1)}(0)=X_{i j}^{\mathrm{v}(2)}(0)=0$.

Pour tenir compte de l'augmentation progressive avec la consolidation cyclique d'une part, du module tangent d'écrouissage et d'autre part de la composante cinématique déterminée par la perte de linéarité des boucles de traction-compression, on prend $C(v)$ sous la forme :

$$
C(v)=C^{(n)}\left(C_{\mathrm{s}}+\left(1-C_{\mathrm{s}}\right) \exp -b v\right),
$$

avec $C_{\mathrm{s}}<1$ et où $C^{(n)}$ sont des constantes.

On illustre sur la figure 21 , dans le cas de l'inoxydable 17-12 SPH, l'augmentation de la composante cinématique avec la déformation au cours d'un essai monotone et au cycle stabilisé d'essais cycliques. On constate effectivement qu'aux cycles stabilisés, les valeurs sont supérieures à celles de l'essai monotone correspondant au premier quart de cycle. On note que d'après $(97 \mathrm{c}), \underline{X}^{\mathrm{V}}$ est complètement restaurable, c'est-à-dire qu'il suffit d'attendre suffisamment longtemps à contrainte nulle, du moins pour les températures intermédiaires car $\beta$ est thermiquement activé, pour voir disparaître tout effet cinématique, ce qui ne reflète pas parfaitement la réalité expérimentale. Une des propriétés caracté- ristiques de l'écrouissage cinématique non linéaire est que, si l'on néglige les termes dépendant du temps et pour un chargement $1 \mathrm{D}$, on peut intégrer analytiquement une équation du type (35) (ou (97c)) sur chaque branche d'un cycle et obtenir la valeur stabilisée $X^{\mathrm{v} \text { stab. }}$ de cette variable, soit :

$$
X^{\mathrm{v} \text { stab. }}=\frac{2}{3} \frac{a^{\mathrm{v}}}{C(v)} \tanh C(v)\left(\frac{\Delta \varepsilon^{\mathrm{v}}}{2}\right) .
$$

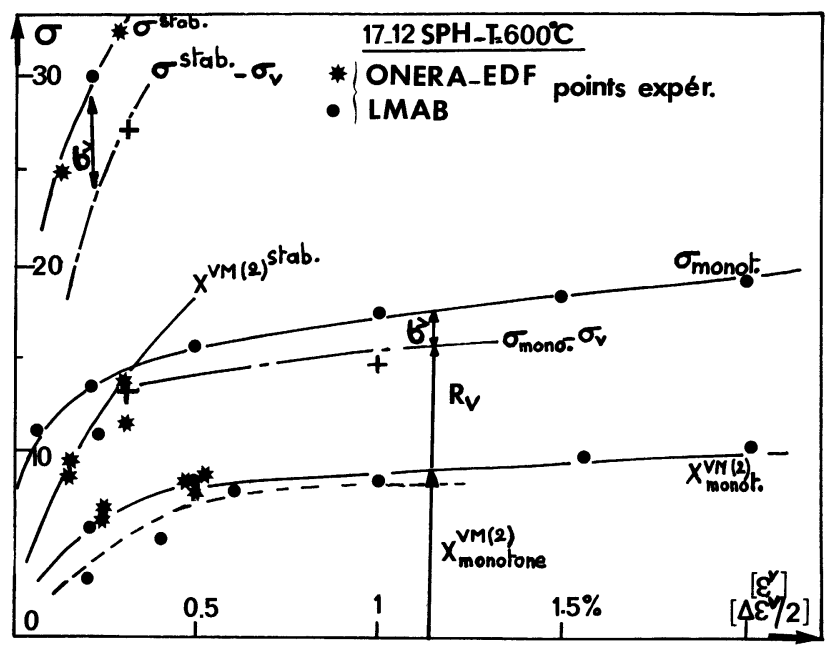

Fig. 21. - Acier inoxydable $17-12 \mathrm{SPH}$ à $T=600^{\circ} \mathrm{C}$; évolutions simultanées de $X^{\mathrm{v}}$ et $\sigma$ en fonction de la déformation pour des chargements monotone et cyclique. On note que la valeur de $X^{\mathrm{v}}$ au cycle stabilisé est supérieure à celle du premier quart de cycle. (D'après Delobelle [47]).

[17-12 SPH stainless at $T=600^{\circ} \mathrm{C}$. Simultaneous variations of $X^{\mathrm{v}}$ and $\sigma$ versus strain for monotonic and cyclic loadings. Notice that the $X^{\mathrm{v}}$ value at the stabilized cycle is larger than that of the first quarter of a cycle. (From Delobelle [17]).]

On comprend l'augmentation de la latitude d'ajustement des courbes cycliques en discrétisant $X^{\mathrm{v}}$ (Eq. (97a)), sa valeur à saturation étant donnée, d'après $(97 \mathrm{c})$, par :

$$
X^{\mathrm{v} \text { stab. }}=\sum_{n=1}^{2} \frac{2}{3} \frac{a^{(n)}}{C(v)} \tanh C(v)\left(\frac{\Delta \varepsilon^{\mathrm{v}}}{2}\right) .
$$

En ce qui concerne la variable isotrope, si $A^{\mathrm{v}}$ est indépendant de $r^{\mathrm{v}}$, d'après les relations (25), (27), on a :

$$
\dot{R}^{v}=\dot{W}^{\prime}\left(r^{v}\right)=\dot{W}^{\prime}\left(\dot{v}-\frac{\partial \Omega_{\mathrm{r}}}{\partial R^{v}}\right) .
$$

En choisissant

$$
W^{\prime}\left(r^{v}\right)=Q_{1}\left[1-\exp -b r^{v}\right]
$$


on arrive à l'équation cinétique :

$$
\dot{R}^{\mathrm{v}}=b\left(Q_{1}-R^{\mathrm{v}}\right)\left(\dot{v}-\frac{\partial \Omega_{\mathrm{r}}}{\partial R^{\mathrm{v}}}\right),
$$

avec la condition $R^{\mathrm{v}}(0)=0$. Dans $(102), b$ est une constante.

Dans le modèle ONERA, la formule (102) est particularisée sous la forme :

$\dot{R}^{\mathrm{v}}=b\left(Q_{1}-R^{\mathrm{v}}\right) \dot{v}-\gamma\left|R^{\mathrm{v}}-Q_{2}\right|^{\ell} \frac{\left(R^{\mathrm{v}}-Q_{2}\right)}{\left|R^{\mathrm{v}}-Q_{2}\right|}$,

où $\gamma$ et $\ell$ sont deux constantes. $Q_{2}$ représente la valeur maximale non restaurable de cette variable isotrope, c'est-à-dire qu'après un écrouissage et un vieillissement suffisamment long à contrainte nulle, $R^{\mathrm{v}}$ tend vers $Q_{2}$. D'autre part, suivant le signe de $\left(R^{\mathrm{v}}-Q_{2}\right)$, on aura restauration par le temps $\left(\left(R^{\mathrm{v}}-Q_{2}\right)>0\right)$ ou écrouissage par le temps $\left(\left(R^{\mathrm{v}}-Q_{2}\right)<0\right)$. Ce dernier cas correspond le plus souvent à des phénomènes d'instabilités structurales uniquement fonction du temps (phénomènes d'interaction à courte distance dislocations-défauts ponctuels au voisinage de $0,25 T_{\mathrm{F}}$ et migration à longue distance de ces mêmes défauts engendrant la précipitation pour les températures plus élevées ; à partir de $0,5 T_{\mathrm{F}}$ ). L'introduction de $Q_{2}$ avec $R^{\mathrm{v}}>Q_{2}$, est tout à fait justifiée au regard d'expériences telles que celles de la figure 10, où lorsque $t_{\mathrm{m}}$ est grand l'amplitude de $\Delta \sigma_{\mathrm{r}}$ tend vers une limite $\Delta \sigma_{\mathrm{r}}(\infty)$, ce qui permet d'accéder à $Q_{2}$. En effet, au cours d'un maintien à contrainte et vitesse nulles $\left(\sigma_{v}=0\right)$ et pour un temps suffisamment long, $\underline{x}^{\mathrm{v}}$ n'a pas de seuil et tend vers zéro, le nouveau seuil d'écoulement sera égal à $\sigma=R_{0}^{\mathrm{v}}+Q_{2}$, d'où $Q_{2}$. On observe sur la figure 10 que $\Delta \sigma_{\mathrm{r}}$ (directement liée à $\left.Q_{2}\right)$, est non seulement fonction de la température mais également de la déformation et ne pourra donc être traitée comme une constante. A température élevée ( $T \geqq 850^{\circ} \mathrm{C}$ dans le cas d'un inoxydable 316 [73]), l'alliage peut recristalliser et perdre totalement sa mémoire (aux effets structuraux près) et ce cas correspond à $Q_{2}=0$.

En l'absence de restauration, pour un chargement monotone $1 \mathrm{D}$, l'intégration de (102) donne, comme pour l'écrouissage cinématique, une cinétique du premier ordre du type Voce, soit :

$$
R^{\vee}=Q_{1}\left(1-\exp -b \varepsilon^{\mathrm{v}}\right) .
$$

Par contre, pour un chargement cyclique, l'état stabilisé de cette variable correspond à :

$$
R^{\mathrm{vstab} .}=Q_{1} \text {. }
$$

Ainsi, dans ce modèle, en l'absence de restauration, la contrainte au cycle stabilisé est donc égale à :

$$
\frac{\Delta \sigma}{2}=R_{0}^{\mathrm{v}}+Q_{1}+X^{\mathrm{vstab} .}+\sigma_{\mathrm{v}},
$$

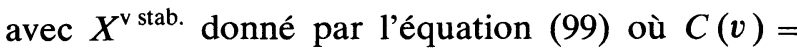
$C_{\mathrm{s}} C^{(n)}$.

On peut observer qu'à l'exemple de la variable $\underline{X}^{v}$ (Eq. (97a)), il est toujours possible d'augmenter le degré d'ajustement du modèle, au détriment de sa simplicité, en écrivant [16] : $R^{\mathrm{v}}=\sum_{n} R^{\mathrm{v}(n)}$. Pour les deux alliages présentés, cette potentialité n'a pas été utilisée.

Cependant, avec une formulation du type (106), au cycle stabilisé sous une amplitude $\left(\Delta \varepsilon_{1}^{\mathrm{T}}\right), R^{\mathrm{v}}$ est saturé à $Q_{1}$ et si l'on augmente l'amplitude du cyclage à $\left(\Delta \varepsilon_{2}^{\mathrm{T}}\right),\left(\Delta \varepsilon_{2}^{\mathrm{T}}>\Delta \varepsilon_{1}^{\mathrm{T}}\right)$, il ne peut plus apparaître une nouvelle consolidation $\left(R^{\mathrm{v}}\right.$ fixé à $\left.Q_{1}\right)$, ce qui est contraire à l'expérience (Fig. 9 par exemple). En adoptant la démarche inverse, c'est-à-dire stabilisation sous $\left(\Delta \varepsilon_{2}^{\mathrm{T}}\right)$ puis réduction de l'amplitude à $\left(\Delta \varepsilon_{1}^{\mathrm{T}}\right)$, l'équation (106) présente les mêmes lacunes qu'aux niveaux croissants $\left(Q_{1}=R^{\mathrm{v}}\right)$, ce qui est également faux, puisqu'expérimentalement, les petits cycles restent momentanément accrochés sur le plus grand cycle stabilisé pour ensuite décroître continuement (Fig. 9). On peut signaler qu'une campagne récente d'essais de rochet biaxé de traction-torsion sur le 17-12 SPH [74], à différentes températures et en fonction de la période de cyclage, de l'amplitude $\Delta \varepsilon^{\mathrm{T}}$ et de son histoire (niveaux successivement croissants puis décroissants), révèle clairement que, même aux plus faibles températures où les effets de restauration sont négligeables, la mémoire du matériau caractérisée par la contrainte de torsion stabilisée et la vitesse de rochet axial cumulée, est totalement évanescente après un nombre de cycles suffisamment grand $(N \geqq 500)$ et ceci indépendamment de la période du cyclage. Le retour à l'équilibre, après un précyclage sous une plus grande amplitude, ne dépend que du nombre de cycles donc uniquement de la déformation cumulée, aucun effet de la période n'est visible.

Toutes ces observations ne sont pas rendues par la relation (106) qui exprime que pour une amplitude donnée, le cycle stabilisé ne dépend pas de l'histoire antérieure.

Pour remédier à ces défauts, il faut que $Q_{1}$ dépende de l'histoire antérieure du matériau et en particulier mémorise la déformation plastique maximale. Ainsi, les auteurs proposent l'introduction d'une nouvelle variable de mémoire $q$, associée à une surface de non-écrouissage $G$, exprimée dans l'espace des déformations $\varepsilon_{i j}^{\mathrm{v}}[16]$, soit :

$$
\left.\begin{array}{l}
G=I_{2}\left(\underline{\varepsilon}^{\mathrm{v}}-\underline{\xi}\right)-q \leqq 0 \\
I_{2}=\sqrt{\frac{2}{3}}\left(\left(\underline{\boldsymbol{\varepsilon}}_{i j}^{\mathrm{v}}-\underline{\xi}_{i j}\right)\left(\varepsilon_{i j}^{\mathrm{v}}-\underline{\xi}_{i j}\right)\right)^{1 / 2}
\end{array}\right\}
$$


A l'exemple des surfaces seuils plastiques, $G$ ne peut évoluer que lorsque $\underline{\varepsilon}^{\mathrm{v}}$ se situe sur la surface $G$, ce qui s'exprime par la relation de consistance $G=\dot{G}=0$. A l'intérieur de cette surface $\dot{q}=0$. Si $\underline{\text { II }}$ et 플 sont respectivement les normales extérieures aux surfaces $f$ (dans l'espace des contraintes) et $G$ (dans l'espace des déformations), soit :

$$
\underline{\underline{n}}=\sqrt{\frac{3}{2}} \frac{\sigma_{i j}^{\prime}-X_{i j}^{\mathrm{v}^{\prime}}}{J_{2}\left(\underline{\mathbf{\sigma}}-\underline{\mathbf{X}}^{\mathrm{v}}\right)}, \underline{\underline{\underline{m}}} *=\sqrt{\frac{2}{3}} \frac{\varepsilon_{i j}^{\mathrm{v}}-\xi_{i j}}{I_{2}\left(\underline{\varepsilon}^{\mathrm{v}}-\underline{\xi}\right)},
$$

on pose d'une façon générale :

$$
\dot{q}=\eta H(G)\left\langle{\underline{n n^{*}}}^{*} \dot{v}-(1-H(G)) g q \dot{v} .\right.
$$

$\left(H\left(_{-}\right)\left\langle_{-}\right\rangle\right.$, a la même signification que précédemment). La mémorisation est effectuée par le premier terme, avec $H(G)=1$, lorsque $\underline{\varepsilon}^{\mathrm{v}}$ est sur $G$, et à l'opposé, l'évanescence de la mémoire est obtenue par le second terme, avec $H(G)=0$, lorsque $\underline{\boldsymbol{\varepsilon}}^{\mathrm{v}}$ est à l'intérieur de $G$. De la relation de consistance, on déduit :

$$
\begin{aligned}
\dot{\xi}_{i j}=\sqrt{\frac{3}{2}\{(1-\eta)} H(G)\langle\underline{\underline{n ! n}} *\rangle \underline{\mathrm{n}}^{*} \dot{v}+ \\
\left.+(1-H(G)) \underline{\mathrm{n}}^{*} g q \dot{v}\right\} .
\end{aligned}
$$

Pour un chargement cyclique uniaxé, avec $H(G)=1$, (109) se réduit à

$$
q=\eta v
$$

Dans la formulation initiale de Chaboche et al. [16], $\eta=\frac{1}{2}$ de sorte que $q=\frac{\Delta \varepsilon_{\mathrm{Max}}^{\mathrm{v}}}{2}$, la mémoire est établie à trois quarts de cycle. L'introduction du paramètre $\eta$, tel que $0 \leqq \eta \leqq 0,5$, est due à Ohno [74] et produit une certaine progressivité dans l'établissement de la mémoire. Si $H(G)=0$, le retour progressif de la mémoire au niveau de celle de la plus faible déformation s'effectue exponentiellement $(\exp -g v)$, la rapidité étant fixée par le paramètre $g$.

Notons que Murakami et Ohno [70] ont également postulé l'existence d'une telle surface pour rendre compte, d'une façon plus réaliste, des réponses aux inversions du sens de chargement à l'aide du modèle isotrope d'écrouissage par la déformation (Eq. (85a)).

Il reste à relier la variable mémoire $q$ à $Q_{1}$, ce qui peut être réalisé simplement en écrivant :

$\dot{Q}_{1}=2 \eta \mu\left(Q_{1}^{\text {sat }}-Q_{1}\right) \dot{q} \quad$ avec $\quad \dot{Q}_{1}(0)=0$.

En 1D, avec $\eta=\frac{1}{2}$, l'équation (112) s'intègre facilement :

$Q_{1}=Q_{1}^{\text {sat }}+\left(Q_{0}-Q_{1}^{\text {sat }}\right) \exp -\mu\left(\frac{\Delta \varepsilon_{\text {Max }}^{\mathrm{v}}}{2}\right)$, et permet d'accéder directement à la valeur de la variable isotrope au cycle stabilisé : $R^{\mathrm{v} \text { sat. }}=Q_{1}$. Il suffit d'introduire (113) dans la relation (106) pour calculer la courbe d'écrouissage cyclique tout en sachant que les multiples effets de mémoire sont réellement pris en compte. Comme nous l'avons évoqué précédemment $Q_{2}$ est une valeur maximale non restaurable par le temps mais fonction de la déformation (effet de mémoire) ce qui peut être traité en corrélant $Q_{2}$ à $Q_{1}$. Les auteurs choisissent la forme :

$$
Q_{2}=Q_{1}-Q_{\mathrm{r}}\left[1-\left(\frac{Q_{1}^{\mathrm{sat}}-Q_{1}}{Q_{1}^{\mathrm{sat}}}\right)^{\ell}\right],
$$

où $Q_{\mathrm{r}}$ est une constante. L'expression (114) est déduite de l'analyse expérimentale de la relaxation cyclique avec temps de maintien $t_{\mathrm{m}}$ au maximum du cycle (par exemple, les résultats de la Fig. 12). En effet, en l'absence de maintien $\left(t_{\mathrm{m}}=0\right)$, on observe dans le cas du 17-12 SPH [76], qu'il existe indépendamment de l'amplitude de cyclage $\left(\Delta \varepsilon^{\mathrm{T}} / 2\right)$, une courbe maîtresse, $\frac{\sigma-\sigma_{0}}{\sigma_{\infty}-\sigma_{0}}=f(v)_{\mathrm{T}}$, où $\sigma_{0}$ est la contrainte maximale au premier cycle, $\sigma_{\infty}$ la contrainte maximale au cycle stabilisé et $\sigma$ la contrainte au cycle considéré. En négligeant le terme de restauration dans l'équation (103) et en considérant que les valeurs maximales de $X^{\mathrm{v}}$ sont constantes, l'intégration de (103) conduit effectivement à une courbe maîtresse [76], soit :

$$
\frac{\sigma-\sigma_{0}}{\sigma_{\infty}-\sigma_{0}} \simeq \frac{R^{\mathrm{v}}-R^{0 \mathrm{v}}}{Q_{1}-R^{0 \mathrm{v}}}=1-\exp -b v .
$$

Par contre, la même analyse, pour $\Delta \varepsilon^{\mathrm{T}}$ fixée mais avec temps de maintien, montre l'existence d'une courbe caractéristique pour chaque $t_{\mathrm{m}}$, ainsi, la relation (115) n'est plus vérifiée. L'analyse de la translation de ces courbes en fonction de $t_{\mathrm{m}}$, conduit, dans le cas du 17-12 SPH, à l'équation (114). $Q_{\text {r }}$ est définie comme la contrainte résiduelle, $Q_{\mathrm{r}}=Q_{2}-Q_{1}$, lorsque $\Delta \varepsilon^{\mathrm{v}} / 2$ est grand.

Du point de vue de l'identification du potentiel de restauration $\Omega_{\mathrm{r}}$ de l'équation (32): $\Omega_{\mathrm{r}}=\Omega_{\mathrm{r}}\left(J_{2}\left(\underline{\mathbf{X}}^{\mathrm{v}}\right)\right.$, $\left.R^{\mathrm{v}}, T\right)$, d'après les équations (97), (102), (103) et (114) en prenant $Q_{\mathrm{r}}=0$, on obtient facilement :

$$
\Omega_{\mathrm{r}}=\frac{\beta}{a^{\mathrm{v}}(r+1)}\left(J_{2}\left(\underline{\mathbf{X}}^{\mathrm{v}}\right)\right)^{r+1}+\frac{\gamma}{b^{\ell}}\left|R^{\mathrm{v}}-Q_{1}\right|^{\ell} .
$$

Dans le cas général $\left(Q_{\mathrm{r}} \neq 0\right)$, en identifiant (102) et (103), on arrive à :

$$
\frac{\partial \Omega_{\mathrm{r}}}{\partial R^{\mathrm{v}}}=-\frac{\gamma}{b} \frac{\left|R^{\mathrm{v}}-Q_{2}\right|^{\ell}}{R^{\mathrm{v}}-Q_{1}} \operatorname{sign}\left(R^{\mathrm{v}}-Q_{2}\right),
$$

qui n'est intégrable que si l'exposant $\ell$ est connu. Par exemple, pour l'acier inoxydable considéré, $\ell=2$ et 
le second terme de l'équation (116) se réécrit :

$$
\begin{aligned}
& \left\{-\frac{\gamma}{2 b}\left(R^{\mathrm{v}}-Q_{1}\right)^{2}-\frac{\gamma}{b}\left(Q_{1}-Q_{2}\right)\left[\left(Q_{1}-Q_{2}\right) \times\right.\right. \\
& \left.\left.\quad \times \operatorname{Ln}\left(R^{\mathrm{v}}-Q_{1}\right)-2\left(R^{\mathrm{v}}-Q_{1}\right)\right]\right\} \operatorname{sign}\left(R^{\mathrm{v}}-Q_{2}\right),
\end{aligned}
$$

qui pour $Q_{1}=Q_{2}$ conduit évidemment à (116).

D'ores et déjà, on s'aperçoit que la complexité de ce modèle n'est que la traduction de la très grande diversité des réponses expérimentales à des sollicitations unidimensionnelles très variées mais judicieusement choisies et permettant de séparer les effets dus à l'écrouissage et au temps.

5.3.2 Le modèle LMAB. - D'après les équations (54), dans l'état actif lorsque $F=0$, on a : $X_{i j}^{\mathrm{v}^{\prime}}=X_{i j}^{\mathrm{vM}}=X_{i j}^{\mathrm{vM}(1)^{\prime}}+X_{i j}^{\mathrm{vM}(2)^{\prime}}$, et dans l'état passif $(F<0), X_{i j}^{\mathrm{v}^{\prime}}=X_{i j}^{\mathrm{vm}^{\prime}} \cdot X_{i j}^{\mathrm{vM}(2)}$ est passible d'une cinétique non linéaire à mémoire évanescente et $X_{i j}^{\mathrm{vM}(1)}$ est liée à l'évolution de la fonction scalaire $R^{\mathrm{v}}$ par l'intermédiaire de l'équation de consistance donnant la relation (54e). Dans l'état de microdéformation, $X_{i j}^{\mathrm{vm}}$ possède en première approximation une cinétique linéaire avec effet de restauration. Pour une meilleure compréhension de la présentation, nous posons a priori les lois d'évolution des variables, puis ensuite nous expliquons la démarche qui a conduit à une telle formulation. Les cinétiques des variables cinématiques sont :

$$
\begin{gathered}
\dot{X}_{i j}^{\mathrm{VM}(2)^{\prime}}=p_{2}\left(\frac{2}{3} R^{\mathrm{v}} \dot{\varepsilon}_{i j}^{\mathrm{v}}-X_{i j}^{\mathrm{vM}(2)^{\prime}} \dot{v}\right)- \\
-p_{2} R_{2}\left(R^{\mathrm{v}}-J_{2}\left(\underline{\mathbf{X}}^{\mathrm{VM}(2)}\right)\right)\left(J_{2}\left(\underline{\underline{\mathbf{X}}}^{\mathrm{vM}(2)}\right)-J_{2}\left(\underline{\mathbf{X}}_{0}^{\mathrm{vM}(2)}\right)\right) \times \\
\times \frac{X_{i j}^{\mathrm{vM}(2)^{\prime}}}{J_{2}\left(\underline{\mathbf{X}}^{\mathrm{vM}(2)}\right)} \\
\dot{X}_{i j}^{\mathrm{VM}(1)^{\prime}}=\frac{\dot{R}^{\mathrm{v}}}{R^{\mathrm{v}}} X_{i j}^{\mathrm{vM}(1)^{\prime}},
\end{gathered}
$$

(voir Eq. (46)) avec,

$\dot{R}^{\mathrm{v}}=b\left(Q_{1}-R^{\mathrm{v}}\right)\left(\dot{v}-R_{1}\left|R^{\mathrm{v}}-Q_{2}\right|^{l} \frac{R^{\mathrm{v}}-Q_{2}}{\left|R^{\mathrm{v}}-Q_{2}\right|}\right)$.

Les conditions initiales sont respectivement :

$$
X_{i j}^{\mathrm{vM}(2)}(0)=0 \text { et } J_{2}\left(\underline{\mathbf{X}}^{\mathrm{vM}(1)}(0)\right)=R^{\mathrm{v}}(0)=R_{0}^{\mathrm{v}} .
$$

Dans l'état passif donnant des microdéformations, on écrira :

$\dot{X}_{i j}^{\mathrm{vm}^{\prime}}=A\left(\frac{2}{3} \dot{\varepsilon}_{i j}^{\mathrm{v}}-R_{0}\left(\sinh \beta^{\prime} J_{2}\left(\underline{\mathbf{X}}^{\mathrm{vm}}\right)\right)^{M} \frac{X_{i j}^{\mathrm{vm}}}{J_{2}\left(\underline{\mathbf{X}}^{\mathrm{vm}}\right)}\right)$

$\mathrm{Ce}$ système d'équations nécessite de nombreux commentaires.
Dans le terme d'écrouissage de l'équation (117a), il apparaît la fonction scalaire $R^{\mathrm{v}}$, c'est-à-dire qu'en l'absence de restauration, $J_{2}\left(\underline{\underline{X}}^{\mathrm{vM}(2)}\right)$ tend vers $R^{\mathrm{v}}$. En effet, en 1D, l'intégration du premier membre de (117) donne :

$$
X^{\mathrm{vM}(2)}=R^{\mathrm{v}}+\left(X^{\mathrm{vM}(2)}(0)-R^{\mathrm{v}}\right) \exp -p_{2} \varepsilon^{\mathrm{v}}
$$$$
\text { (modèle de Voce), }
$$

et si $p_{2}$ est grand, $X^{\mathrm{vM}(2)}$ tend rapidement vers $R^{v}$. Cette modélisation dérive de l'observation que pour un chargement monotone ou durant la consolidation cyclique, si l'on mesure $X^{\mathrm{vM}(2)}$ (correspondant aux maximas des cycles) par la perte de linéarité des boucles de traction-compression (pour un offset donné de l'ordre de $5 \times 10^{-4}$ ), on constate que l'on vérifie sensiblement la propriété [77] :

$$
\sigma-\sigma_{\mathrm{v}} \simeq 2 X^{\mathrm{vM}(2)} \simeq 2 R^{\mathrm{v}},
$$

(Fig. 21 et 22 par exemple). $\sigma_{\mathrm{v}}$ est mesurée par la méthode de la relaxation inverse évoquée en (5.2.2). Ceci revient à écrire que dans le plan du déviateur des contraintes internes (Fig. 14) (ou des contraintes appliquées si $\sigma_{\mathrm{v}}$ est faible), la surface $F=0$ passe au voisinage de l'origine $\left(X_{k l}^{\prime}=0\right)$, ce qui se vérifie aisément sous sollicitation cyclique uniaxée où l'on observe que la perte de linéarité des boucles correspond sensiblement à l'axe $\sigma_{z z}=0$ (cf. 3.2.2 et Fig. 6). Sous chargement biaxé, les surfaces de charge obtenues par Ellis et coll. [22], sur un acier inoxydable 316 à l'ambiante ( $\sigma_{\mathrm{v}}$ faible), corroborent cette propriété : outre leur grossissement et leur translation, elles passent toujours très près de

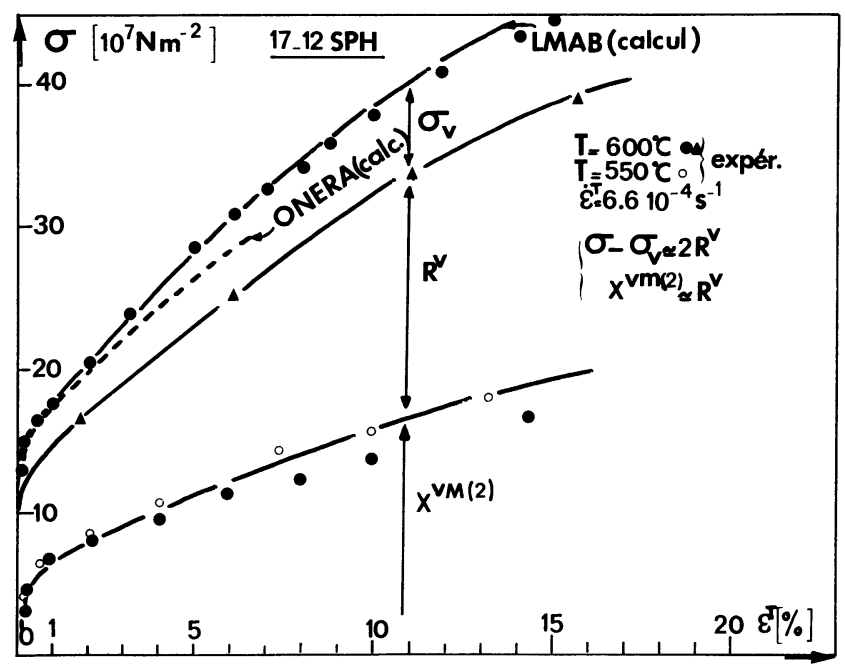

Fig. 22. - Acier 17-12 SPH ; vérification de la relation empirique (121), $\sigma-\sigma_{\mathrm{v}} \simeq 2 X^{\mathrm{vM}(2)}$, pour un essai monotone. (D'après Delobelle [7], thèse France).

[17-12 SPH. Checking of empirical relationship (21), $\sigma-\sigma_{\mathrm{v}} \simeq 2 X^{\mathrm{vM}(2)}$ for a monotonic test. (From Delobelle [7], thesis, France).] 
l'origine des contraintes. Ainsi, l'augmentation progressive de $\underline{X}^{\mathrm{vM}(2)}$ au cours de la consolidation cyclique est en partie prise en compte par la croissance de $R^{\mathrm{v}}$ avec la déformation viscoplastique cumulée $v$. En effet, d'après la relation (98), au cycle stabilisé, on a :

$$
X^{\mathrm{vM}(2) \text { stab. }}=R^{\mathrm{v}} \tanh p_{2}\left(\frac{\Delta \varepsilon^{\mathrm{v}}}{2}\right)
$$

et si $p_{2}$ est grand, en fonction de l'amplitude du cyclage $\left(\frac{\Delta \varepsilon^{T}}{2}\right)$ on tend rapidement vers : $X^{\mathrm{vM}(2) \text { stab. }} \simeq R^{\mathrm{v} \text { tab. }}=Q_{1}$. La courbe cyclique est donc accessible, en tenant compte de l'équation de consistance (46) en $1 \mathrm{D}, X^{\mathrm{vM}(1)}= \pm R^{\mathrm{v}}$, par :

$$
\frac{\Delta \sigma}{2}=X^{\mathrm{vM} \mathrm{stab} .}+\sigma_{\mathrm{v}}=Q_{1}+X^{\mathrm{vM}(2) \mathrm{stab} .}+\sigma_{\mathrm{v}},
$$

qui, pour des cycles assez grands, conduit à :

$$
\frac{\Delta \sigma}{2} \simeq 2 Q_{1}+\sigma_{\mathrm{v}}
$$

d'où la relation empirique (121).

Il en est de même pour les courbes monotones, soit :

$$
\sigma=R^{\mathrm{v}}+X^{\mathrm{vM}(2)}+\sigma_{\mathrm{v}}
$$

qui, pour $\Delta \varepsilon^{\vee}$ assez grand redonne (121) :

$$
\sigma \simeq 2 R^{\mathrm{v}}+\sigma_{\mathrm{v}} .
$$

Un autre effet également restitué par l'introduction de $R^{\mathrm{v}}$ dans le coefficient d'écrouissage de $\underline{\mathrm{X}}^{\mathrm{vM}(2)}$ est l'augmentation au cours du cyclage du module plastique tangent pour une déformation fixée. De même, la bilinéarité très prononcée des courbes de traction monotones (Fig. 1) est également appréhendée puisque $X^{\mathrm{vM}(2)}$ tend rapidement ( $p_{2}$ grand) vers $X^{\mathrm{vM}(1)}=R^{\mathrm{v}}$ qui évolue lentement ( $b$ faible) avec la déformation.

On note que dans la version originale du modèle $[7,9]$, on faisait abstraction de la propriété (121) et l'on introduisait d'une manière générale une fonction scalaire $X^{(2) \text { sat }}$ telle que :

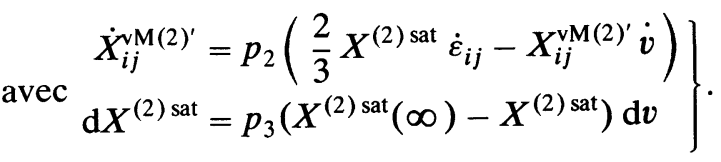

L'équation (117) n'est qu'un cas particulier de (125) avec $X^{(2) \text { sat }}=R^{\mathrm{v}}$ d'où $p_{3}=b$ et $X^{(2) \text { sat }}(\infty)=$ $Q_{1}$.

On montre la cohérence qualitative des formulations ONERA et LMAB en identifiant les relations $(97 \mathrm{c})$ et $(117 \mathrm{a})$, ce qui conduit à :

$$
R^{v}=a^{(n)}[C(v)]^{-1}
$$

On constate effectivement que $R^{\mathrm{v}}$ (Eq. (118)) et $C(v)(E q$. (97d)) sont respèctivement des cinétiques du premier ordre croissante et décroissante, d'où l'aptitude des deux modèles à reproduire les différentes propriétés cycliques décrites précédemment. De plus, d'après ce modèle, dans le cas d'une déconsolidation cyclique (INCO 718), on a $R^{\mathrm{v}}(0)=R_{0}^{\mathrm{v}}>$ $Q_{1}$ et le module plastique tangent décroît avec la contrainte, ce qui est expérimentalement exact.

D'une manière encore plus aiguë que dans le modèle ONERA, on voit que, d'après (123b), il est crucial, afin de décrire correctement les courbes cycliques et les différents effets de mémoire présentés précédemment, que $Q_{1}$ dépende de l'histoire du matériau et en particulier d'une fonctionnelle de la déformation. Dans le cas contraire, en faisant abstraction de la viscosité et pour des cycles suffisamment grands, la courbe cyclique serait une horizontale donnée par $\frac{\Delta \sigma}{2}=2 Q_{1}$. On postule donc, comme pour le modèle ONERA, l'existence d'une surface de non-écrouissage $G$ donnée par les relations (107), [77] : $G=I_{2}\left(\underline{\varepsilon}^{\mathrm{v}}-\underline{\xi}\right)-q<0$ et telle que :

$$
\dot{q}=\eta H(G)\left\langle\underline{\underline{n}}^{*}\right\rangle \dot{v}-(1-H(G)) g q \dot{v}
$$

(voir Eq. (109)).

On rappelle que $\xi_{i j}$ est obtenue par l'équation de consistance $G=\dot{G}=0$ (Eq. (110)) dans laquelle, pour ce modèle, 트* et 트 sont respectivement les normales extérieures à $G=0$ et aux surfaces équipotentielles $\Omega_{\mathrm{v}}$. Jusqu'à présent, seules les simulations 1D d'essais monotones et cycliques à plusieurs niveaux ont été réalisées, on prendra donc $\eta=\frac{1}{2}$, ce qui permet compte tenu des équations (109) et (112) de calculer directement la courbe de consolidation cyclique. Avec (122) et (123), on aboutit à :

$$
\frac{\Delta \sigma}{2}=Q_{1}\left(1+\tanh p_{2}\left(\frac{\Delta \varepsilon_{\max }^{\mathrm{v}}}{2}\right)\right)+\sigma_{\mathrm{v}}
$$

qui tend très rapidement ( $p_{2}$ grand) vers :

$$
\frac{\Delta \sigma}{2} \simeq 2\left[Q_{1}^{\mathrm{sat}}\left(1-\exp -\mu \frac{\Delta \varepsilon_{\max }^{\mathrm{v}}}{2}\right)\right]+\sigma_{\mathrm{v}} .
$$

On s'aperçoit que ce modèle est essentiellement géré par la fonction scalaire $R^{\mathrm{v}}$, la variable $\underline{\mathrm{X}}^{\mathrm{vM}(2)}$ servant à décrire d'une façon continue les boucles d'hystérésis consécutives aux inversions du sens de la déformation. On peut faire un parallèle avec les modèles de l'IMG [4] et de Krempl [48, 49], où les inversions sont traitées d'une manière discrète, respectivement par des procédures algorithmiques de réinitialisation ou de mise en mémoire d'instants précis du chargement et l'aspect des boucles traités par un comportement de Masing ou par redéfinition 
de la fonction d'équilibre $g(\varepsilon)$. On peut également souligner que la mémorisation de l'instant où la contrainte franchit $g(\varepsilon)$ (modèle de Krempl) est strictement identique (en 1D) au passage macromicrodéformation puisque $g(\varepsilon)=X^{\mathrm{vM}(2)}+R^{\mathrm{v}}$.

Dans l'état passif, en première approximation, on adopte pour la variable cinématique $X_{i j}^{\mathrm{v}}=X_{i j}^{\mathrm{vm}}$, une cinétique linéaire du type Prager, $\dot{X}_{i j}^{\mathrm{vm}}=\frac{2}{3} A \dot{\varepsilon}_{i j}$ (Eq. (119)). Le coefficient $A$ est très grand (de l'ordre des modules anélastiques rencontrés dans les aciers ; 1 à $2 \times 10^{6} \mathrm{MPa}$ ) d'où la faiblesse, en l'absence d'effets liés au temps, des déformations correspondant à l'état passif. De plus, on montre très clairement $[7,10]$ que ces microdéformations vérifient la première règle de normalité (Eq. (18)), d'où le vocable de microviscoplasticité. Ainsi, les faibles déformations négatives de recouvrance sont décrites par ce modèle sans pour autant avoir recourt à la partition des déformations visqueuse et anélastique, comme on la trouve dans de nombreuses publications [52-54, 78]. Une étude détaillée des propriétés de la recouvrance [10] montre qu'elle dépend de l'histoire antérieure du matériau, c'est-àdire que $A$ (Eq. (119)) n'est pas constante. Cependant, pour les applications, compte tenu des ordres de grandeur mis en jeu par ces microdéformations $\left(\varepsilon^{\mathrm{vm}} \leqq 2 \times 10^{-4}\right.$ dans le cas d'un acier 17-12 SPH pour $T \simeq 600^{\circ}$ ), cette omission ne conduit qu'à des erreurs maximales voisines de $10^{-4}$, ce qui peut être négligé. D'un point de vue physique, ces déformations peuvent être attribuées aux variations de la distribution des rayons de courbure des dislocations ancrées, sous l'action des variations du champ de contrainte interne consécutives à une perturbation externe. Ces microdéformations sont donc fonctions de la densité totale des dislocations et l'on comprend aisément qu'elles dépendent de l'histoire antérieure du matériau (donc de l'état actif dans le présent modèle). Comme dans le modèle ONERA, les nombreuses manifestations liées au temps (cf. 3.2.3) sont prises en compte par l'introduction de termes de restauration dans les équations (117a), (118) et (119).

De plus, à l'exemple de l'équation d'état (71a), dans le cas du 17-12 SPH, ces différentes cinétiques ont été identifiées sur une plage de température telle que : $550 \leqq T \leqq 700^{\circ} \mathrm{C}$. Dans ce modèle, les termes de restauration ont été évalués à partir d'une part, de l'étude détaillée du fluage stationnaire [7,9] sous différents niveaux de contrainte et de température (Figs. 3 et 5) et d'autre part, d'essais de restauration de la contrainte d'écoulement après un temps de maintien à contrainte nulle et ceci pour différents états de préécrouissage (Fig. 10). La principale conclusion est l'indifférence de l'état stationnaire à l'histoire antérieure du matériau (Fig. 5 par exemple), c'est-à-dire qu'après une histoire quelconque de sollicitation, la vitesse stationnaire sous le dernier niveau $\sigma_{2}$, atteinte après un laps de temps plus ou moins long, qui lui est fonction de l'histoire, est identique à celle correspondant à un premier chargement sous $\sigma_{2}$. On montre également $[7,60]$ que pour des histoires de chargement biaxées quelconques à $J_{2}(\underline{\boldsymbol{\sigma}})$ constant (traction-torsion), la vitesse stationnaire est unique et vérifie toujours la règle de normalité à des équipotentielles de Von-Misès, ce qui correspond à un retour de la variable cinématique vers la situation des chargements radiaux, à savoir la colinéarité de $\underline{\sigma}^{\prime}$ et de $\underline{\mathbf{X}}^{\prime}$. Durant les périodes d'écoulement transitoire on assiste à une rotation de la direction d'écoulement, fonction de l'histoire antérieure et en particulier de l'angle entre deux points de chargement consécutifs, ce qui peut être interprété comme la manifestation du retour vers les conditions d'équilibre de la variable $\underline{\mathbf{X}}$. Ainsi, dans ce modèle, l'état stationnaire doit être identique dans les états actif ou passif et correspond, pour des chargements multiaxés, à un état radial. Cette propriété explique en partie la forme générale des équations (117a), (118) et (119), qui, pour des chargements monotones radiaux, s'écrit :

$$
\dot{X}_{(n)}=H_{(n)}\left(\dot{\varepsilon}-R_{(n)}\right),
$$

$\left(H_{(n)}\right.$ et $R_{(n)}$ étant respectivement les coefficients d'écrouissage et de restauration), la vitesse $\dot{\varepsilon}^{\text {st }}$, état de référence privilégié pour l'expérimentateur, étant égale à $\dot{\varepsilon}^{\text {st }}=R_{(n)}$.

Dans l'état de microdéformation, l'application d'une part de la méthode de Mitra-McLean [79, 80] pour plusieurs isothermes, c'est-à-dire l'analyse des temps d'incubation (ou période d'hésitation au fluage) pour différents décréments de contrainte à partir de l'état stationnaire et d'autre part, de la méthode de Gasca-Neri et coll. [81], qui consiste à mesurer l'incrément de déformation consécutif au retour de la contrainte à sa valeur nominale après une décharge préalable, conduit à l'écriture de l'équation (119) [7, 9-10]. La vitesse stationnaire s'écrit :

$$
\dot{\varepsilon}^{\mathrm{st}}=R_{0}\left(\sinh \beta^{\prime} X^{\mathrm{vm}}\right)^{\mathrm{M}},
$$

ce qui se vérifie expérimentalement [60] et permet d'expliquer, pour les faibles et moyennes contraintes (où $\left(X^{\mathrm{vM}} / \sigma\right) \simeq 1$ ), le réseau des courbes caractéristiques classiques $\operatorname{Ln} \dot{\varepsilon}^{\text {st }}=f(\operatorname{Ln} \sigma)_{\mathrm{T}}$.

En s'inspirant de la démarche de Whilshire et coll. [82] pour l'analyse de la vitesse stationnaire, on montre que celle-ci s'exprime également sous la forme :

$$
\dot{\varepsilon}^{\mathrm{st}}=R_{3}\left(X^{\mathrm{vM}}-X_{0}^{(\infty)}\right)^{l}
$$

avec

$$
R_{3}=C * \frac{D_{\mathrm{Fe}} G b}{k T},
$$


et où $X_{0}^{(\infty)}$ représente la valeur maximale non restaurable de la variable $X^{\mathrm{vM}}$, déterminée par l'étude de la cinétique de restauration de la contrainte d'écoulement après différents maintiens à contrainte nulle, soit :

$$
X_{0}^{(\infty)}=X^{\mathrm{vM}}-\lim _{t_{\mathrm{m}} \rightarrow \infty}\left(\Delta \sigma_{\mathrm{r}}\right)
$$

L'étude de la vitesse stationnaire sous différents chargements biaxés conduit à l'extension de l'équation (131), soit :

$$
\dot{v}=R_{3}\left(J_{2}\left(\underline{\mathbf{X}}^{\mathrm{vM}}-\underline{\mathbf{X}}_{0}^{(\infty)}\right)^{l}\right) .
$$

Si l'on considère durant l'état stationnaire, d'une part la propriété de colinéarité des tenseurs évoquée précédemment et d'autre part que la vitesse stationnaire correspond à l'état d'équilibre de chacune des variables (Eq. (131a)), $\dot{J}_{2}\left(\underline{\mathbf{X}}^{\mathrm{vM}(2)}\right)=\dot{R}^{\mathrm{v}}$, on montre que :

$$
\begin{aligned}
\dot{v} & =R_{3}\left(J_{2}\left(\underline{\mathbf{X}}^{\mathrm{vM}}\right)-J_{2}\left(\underline{\mathbf{X}}_{0}^{(\infty)}\right)\right)^{l} \\
& =R_{2}\left(J_{2}\left(\underline{\mathbf{X}}^{\mathrm{vM}(2)}\right)-J_{2}\left(\underline{\mathbf{X}}_{0}^{\mathrm{vM}(2)}\right)\right)^{l} \\
& =R_{1}\left(R^{\mathrm{v}}-Q_{2}\right)^{l} \\
& \left(\frac{1}{R_{3}}\right)^{l}=\left(\frac{1}{R_{1}}\right)^{l}+\left(\frac{1}{R_{2}}\right)^{l},
\end{aligned}
$$

avec

d'où l'écriture des termes de restauration des équations (117a) et (118). $\underline{\mathbf{x}}_{0}^{\mathrm{vM}(2)}$ et $Q_{2}$ sont les valeurs maximales non restaurables de $\underline{\mathrm{X}}^{\mathrm{vM}(2)}$ et $R^{\mathrm{v}}$.

Les paramètres $R_{1}$ et $R_{2}$ fixent la vitesse de restauration de chacune des variables tout en respectant l'équation (133) et peuvent être ajustés à l'aide de séquences de vieillissement au cours d'essais de traction-compression ou torsion alternée [9, 17].

L'indifférence de l'écoulement stationnaire dans les deux états du système (Eqs. (130) et (133)) permet de définir les valeurs maximales non restaurables $J_{2}\left(\underline{\mathbf{X}}_{0}^{\mathrm{vM}(2)}\right)$ et $Q_{2}$, soit :

$$
\left.\begin{array}{rl}
Q_{2} & =\operatorname{Max}\left(R^{\mathrm{v}}-\left(R_{0} / R_{1}\right)^{1 / l}\left(\sinh \beta^{\prime}\left(J_{2}\left(\underline{\mathbf{X}}^{\mathrm{vM}(2)}\right)+R^{\mathrm{v}}\right)\right)^{M / l}\right) \\
J_{2}\left(\underline{\mathbf{X}}_{0}^{\mathrm{vM}(2)}\right) & =\left(\operatorname{Max}\left(J_{2}\left(\underline{\mathbf{X}}^{\mathrm{vM}(2)}\right)-\left(R_{0} / R_{2}\right)^{1 / l}\left(\sinh \beta^{\prime}\left(J_{2}\left(\underline{\mathbf{X}}^{\mathrm{vM}(2)}\right)+R^{\mathrm{v}}\right)\right)^{M / l}\right)\right.
\end{array}\right\}
$$

On remarque que les paramètres $R_{1}$ et $R_{2}$ déterminent l'amplitude de $Q_{2}$ et $J_{2}\left(\underline{\mathbf{X}}_{0}^{\mathrm{vM}(2)}\right)$. Généralement, $R_{1} \neq R_{2}$ donc les deux variables sont susceptibles de se restaurer. Ces relations montrent également que $Q_{2}$ et $J_{2}\left(\underline{\mathbf{X}}_{0}^{\mathrm{vM}(2)}\right)$ ne sont pas des paramètres supplémentaires du modèle, toutes les grandeurs dans ces expressions sont connues. La fonction Max. tient compte de ce que l'on décrit des grandeurs non restaurables, qui ne peuvent que croître.

D'un point de vue thermodynamique, l'identification des relations (117), (118) et (119) au système d'équations (51) n'est cohérente que si $B=p_{2} R^{\mathrm{v}}$ et $b^{*}=p_{2}=\frac{Q_{1}-R^{\mathrm{v}}}{R^{\mathrm{v}}}$, la dernière égalité étant très restrictive et ne représente qu'un cas bien particulier du modèle général. En fait, les équations (41) à (52) ne fixent que le contexte thermodynamique global de ce modèle et pour une identification rigoureuse et cohérente des cinétiques aux équations thermodynamiques, il est nécessaire de travailler directement sur chacune des variables, soit $\underline{\boldsymbol{\alpha}}^{\mathrm{vm}}, \underline{\boldsymbol{\alpha}}^{\mathrm{vM}(1)}$ et $\underline{\boldsymbol{\alpha}}^{\mathrm{vM}(2)}$ et non plus sur la variable globale $\underline{\boldsymbol{\alpha}}^{\mathrm{vM}}$ (Eqs. (42b) et (47)) [39]. Le développement total du calcul est très long, donc non reporté, mais repose sur la définition de l'énergie libre sous la forme d'une combinaison linéaire de formes quadratiques de chacune des variables et d'un potentiel de dissipation $(\Omega=$ $\Omega_{\mathrm{v}}+\boldsymbol{\Omega}_{\mathrm{r}}$ ) faisant également apparaître des termes quadratiques d'une part en $\underline{\mathbf{X}}^{\mathrm{vm}}, \underline{\mathbf{X}}^{\mathrm{vM}(1)}$ et $\underline{\mathbf{X}}^{\mathrm{vM}(2)}$ et d'autre part, en $\underline{\boldsymbol{\alpha}}^{\mathrm{vm}}$, $\underline{\boldsymbol{\alpha}}^{\mathrm{vM}(1)}$ et $\underline{\boldsymbol{\alpha}}^{\mathrm{vM}(2)}$.

Comme pour le modèle ONERA, la complexité de cette approche n'est qu'une tentative de traduction de la complexité du comportement des matériaux aux températures intermédiaires. Les différences essentielles résident bien évidemment dans le choix des différentes fonctionnelles mais surtout dans l'existence ou l'absence de zone de non-écoulement et dans le choix de la nature et du nombre des variables (une cinématique $\underline{X}^{v}$ et une isotrope $R_{\mathrm{v}}$ dans le modèle ONERA et trois cinématiques $\underline{\mathbf{X}}^{\mathrm{vm}}, \underline{\mathbf{X}}^{\mathrm{vM(1)}}$ et $\underline{\mathbf{X}}^{\mathrm{vM(2)}}$ pour l'approche du LMAB).

5.3.3 Le modèle de l'EMP. - Puisqu'il s'agit d'un modèle partitionné, conformément aux équations (55f), il faut définir les lois d'évolution du centre et du rayon de chacune des surfaces $f$ et $g$. La formulation thermodynamique évoquée précédemment (Eqs. (37) et (38)), conduit aux lois cinématiques, soit d'après $(40 \mathrm{a})$ :

$$
\left.\begin{array}{rl}
\dot{X}_{i j}^{\mathrm{v}}= & K^{\mathrm{v}}\left(\frac{2}{3} \dot{\varepsilon}_{i j}^{\mathrm{v}}-C^{\mathrm{v}} \dot{v} X_{i j}^{\mathrm{v}}\right)+ \\
& +K^{\mathrm{vp}}\left(\frac{2}{3} \dot{\varepsilon}_{i j}^{\mathrm{p}}-C^{\mathrm{p}} \dot{p} X_{i j}^{\mathrm{p}}\right) \\
\dot{X}_{i j}^{\mathrm{p}}= & K^{\mathrm{p}}\left(\frac{2}{3} \dot{\varepsilon}_{i j}^{\mathrm{p}}-C^{\mathrm{p}} \dot{p} X_{i j}^{\mathrm{p}}\right)+ \\
& +K^{\mathrm{vp}}\left(\frac{2}{3} \dot{\varepsilon}_{i j}^{\mathrm{v}}-C^{\mathrm{v}} \dot{v} X_{i j}^{\mathrm{v}}\right)
\end{array}\right\},
$$

avec

$$
X_{i j}^{\mathrm{v}}(0)=X_{i j}^{\mathrm{p}}(0)=0 \text {. }
$$

Ainsi, le couplage «plasticité-fluage » s'effectue par l'intermédiaire du coefficient $K^{\mathrm{vp}}$, mais on peut déplorer l'absence de terme de restauration sur ces 
deux variables, ce qui constitue une certaine lacune du modèle.

En suivant une démarche analogue à celle développée pour le modèle ONERA (Eqs. (100) à (102)), les variables isotropes sont définies par :

$$
\left.\begin{array}{l}
\dot{R}^{\mathrm{v}}=b^{\mathrm{v}}\left(Q_{1}^{\mathrm{v}}-R^{\mathrm{v}}\right) \dot{v}-\gamma^{\mathrm{v}}\left(R^{\mathrm{v}}\right)^{l} \\
\dot{R}^{\mathrm{p}}=b^{\mathrm{p}}\left(Q_{1}^{\mathrm{p}}-R^{\mathrm{p}}\right) \dot{p}-\gamma^{\mathrm{p}}\left(R^{\mathrm{p}}\right)^{l}
\end{array}\right\},
$$

avec $R^{\mathrm{v}}(0)=R^{\mathrm{P}}(0)=0$. On peut mentionner que les auteurs ont également adopté des conditions initiales telles que : $R^{\mathrm{v}}(0)=R_{0}^{\mathrm{v}}$ et $R^{\mathrm{p}}(0)=R_{0}^{\mathrm{p}}$, mais en supprimant les termes $R_{0}^{\mathrm{v}}$ et $R_{0}^{\mathrm{p}}$ dans les potentiels $f$ et $\Omega$ (Eqs. (55b et c)).

Les deux variables $R^{\mathrm{V}}$ et $R^{\mathrm{p}}$ présentent d'une part, un durcissement cyclique respectivement fonction des déformations cumulées $\dot{v}$ et $\dot{p}$, et d'autre part, des termes d'adoucissement. Cependant, l'adoucisse- ment peut être total puisqu'il n'existe pas de seuils sur les termes de restauration, ce qui constitue également une lacune vis-à-vis des effets intrinsèquement liés au temps (par exemple Figs. 10 et 11) qui possèdent fréquemment des propriétés asymptotiques. Les auteurs postulent également que les déformations purement plastiques peuvent être affectées par des termes uniquement fonction du temps, ce qui rejoint une des hypothèses de base de la première version du modèle du CEA.

Les propriétés monotones et cycliques en $1 \mathrm{D}$ ne sont pas facilement analysables puisque le système d'équations (135) ne présente pas de solution analytique. Cependant, plusieurs cas limites simples peuvent être envisagés. Ainsi, si le couplage est nul $K^{\mathrm{vp}}=0$ et en l'absence de termes de restauration dans les équations (136), la courbe cyclique est donnée par:

$$
\frac{\Delta \sigma}{2}=\left[R_{0}^{\mathrm{v}}+Q_{1}^{\mathrm{v}}+\frac{2}{3 C^{\mathrm{v}}}\left(\tanh K^{\mathrm{v}} C^{\mathrm{v}}\left(\frac{\Delta \varepsilon^{\mathrm{v}}}{2}\right)\right)+\sigma_{\mathrm{v}}\right]+\left[R_{0}^{\mathrm{p}}+Q_{1}^{\mathrm{p}}+\frac{2}{3 C^{\mathrm{p}}} \tanh K^{\mathrm{p}} C^{\mathrm{p}}\left(\frac{\Delta \varepsilon^{\mathrm{p}}}{2}\right)\right],
$$

et où les deux membres entre crochets sont respectivement les contributions visqueuse et plastique (il n'existe aucun couplage entre les deux termes). Un autre cas consiste à prendre tous les coefficients égaux : $K^{\mathrm{p}}=K^{\mathrm{v}}=K^{\mathrm{vp}}=K$ et $C^{\mathrm{v}}=C^{\mathrm{p}}=C$, ce qui implique que $\underline{\dot{\mathbf{x}}}^{\mathrm{p}}=\underline{\dot{\mathbf{x}}}^{\mathrm{v}}=\underline{\dot{\mathbf{x}}}$, d'où la description de la courbe cyclique :

$$
\begin{aligned}
\frac{\Delta \sigma}{2}=R_{0}^{\mathrm{v}}+ & R_{0}^{\mathrm{p}}+Q_{1}^{\mathrm{v}}+Q_{1}^{\mathrm{p}}+ \\
& +\frac{2}{3 C} \tanh K C \frac{\Delta\left(\varepsilon^{\mathrm{p}}+\varepsilon^{\mathrm{v}}\right)}{2}+\sigma_{\mathrm{v}} .
\end{aligned}
$$

Le couplage est total et l'on se retrouve dans une situation voisine de celle des deux modèles unifiés mais où la déformation est la somme des composantes visqueuse et plastique (modèle partitionné). Lorsque $K^{\mathrm{vp}} \neq 0$, la solution réside entre ces deux cas extrêmes. Une analyse identique pourrait être faite pour les courbes monotones. A ce stade, on peut remarquer que la solution de couplage préconisée par Ohashi et al. [71] (Eq. (90)) est analytiquement résoluble et la valeur stabilisée de la variable cinématique correspond à :

$$
X^{\mathrm{pstab} .}=\frac{K}{C} \tanh C \frac{\Delta\left(\varepsilon^{\mathrm{p}}+\beta^{+} \varepsilon^{\mathrm{v}}\right)}{2} .
$$

Le couplage s'effectue donc par le coefficient $\beta^{+}$.

Les différents effets de mémoire de la prédéformation affectant les propriétés cycliques et évoqués précédemment ainsi que l'évolution continue du module tangent au cours du cyclage ne sont pas pris en compte par ce modèle. Puisque ces phénomènes sont toujours visibles même en l'absence d'effet de viscosité, on suggère de modifier uniquement les variables $\underline{\underline{X}}^{\mathrm{p}}$ et $R^{\mathrm{p}}$. A l'exemple des modèles ONERA et $\mathrm{LMAB}$, on prendra soit $C^{\mathrm{p}}$ fonction décroissante de $p$, soit l'on introduira un coefficient multiplicatif à $\dot{\varepsilon}_{i j}^{\mathrm{p}}$, dans la cinétique de $\underline{\dot{\mathbf{x}}}^{\mathrm{p}}$, proportionnel à $\left[C^{\mathrm{p}}\right]^{-1}$ (Eq. (126)). De plus, on préconise l'introduction d'une surface de non-écrouissage plastique $G$ (Eq. (107)), soit :

$$
G=I_{2}\left(\underline{\varepsilon}^{\mathrm{p}}-\underline{\xi}^{\mathrm{p}}\right)-q^{\mathrm{p}} \leqq 0,
$$

et de sorte que $\dot{Q}_{1}^{\mathrm{p}}$ dépende de $\dot{q}^{\mathrm{p}}$ d'une façon similaire à celle de l'équation (112). Moyennant ces deux modifications, ce modèle devrait décrire correctement l'ensemble des différentes propriétés cycliques.

Du point de vue thermodynamique, les équations cinétiques dérivent des potentiels $\Omega_{\mathrm{v}}$ et $f(\mathrm{Eq}$. (37)) auxquels on adjoint les potentiels de restauration $\Omega_{\mathrm{r}}\left(R^{\mathrm{v}}\right)$ et $f_{\mathrm{r}}\left(R^{\mathrm{p}}\right)$ (Eq. (55)). En calquant la démarche adoptée pour les relations (100) à (103), on définit $\Omega_{\mathrm{r}}$ et $f_{\mathrm{r}}$ par:

$$
\frac{\partial \Omega_{\mathrm{r}}}{\partial R^{\mathrm{v}}}=\frac{\gamma^{\mathrm{v}}}{b^{\mathrm{v}}} \frac{R^{\mathrm{v}^{l}}}{Q_{1}^{\mathrm{v}}-R^{\mathrm{v}}} \quad \text { et } \quad \frac{\partial f_{\mathrm{r}}}{\partial R^{\mathrm{p}}}=\frac{\gamma^{\mathrm{p}}}{b^{\mathrm{p}}} \frac{R^{\mathrm{p}^{l}}}{Q_{1}^{\mathrm{p}}-R^{\mathrm{p}}} .
$$

Comme pour le modèle ONERA, si $l$ est connu, on peut donner une solution explicite pour $\Omega_{\mathrm{r}}$ et $f_{\mathrm{r}}$. Par exemple, dans le cas du 17-12 SPH à $600^{\circ} \mathrm{C}$ les auteurs reportent une valeur $l=2$, ce qui conduit à des solutions du type de celle reportée pour la résolution de l'équation (117).

En conclusion, moyennant les quelques modifica- 
tions proposées, ce modèle devrait être capable de simuler l'ensemble des observations expérimentales 1D. Cependant, il reste difficile à identifier puisqu'il fait appel à quatre variables obéissant à quatre équations différentielles dont deux sont couplées. On peut mentionner que par ordre de difficulté croissante d'identification, ces trois modèles se classent suivant la séquence : ONERA-LMT, LMAB et EMP.

5.3.4 Le modèle multicouche parallèle du CEA. Dans ce modèle, la particularisation du système d'équations (28), s'écrit pour chaque couche :

avec

$$
\left.\begin{array}{rl}
\dot{X}_{i j}^{\mathrm{p}(n)} & =\frac{2}{3} a^{(n)} \dot{\varepsilon}_{i j}-A\left(J_{2}\left(\underline{\mathbf{X}}^{\mathrm{p}(n)}\right)\right)^{r} \frac{X_{i j}^{\mathrm{p}(n)}}{J_{2}\left(\underline{\mathbf{X}}^{\mathrm{p}(n)}\right)} \\
X_{i j(0)}^{\mathrm{p}(n)} & =0 \\
R^{\mathrm{p}(n)} & =R_{0}^{\mathrm{p}(n)}
\end{array}\right\}
$$

On a de plus l'équation d'équilibre du modèle parallèle, soit : $\underline{\mathbf{\sigma}}=\sum_{(n)} w^{(n)} \underline{\mathbf{\sigma}}^{(n)}, \underline{\mathbf{\sigma}}^{(n)}$ étant déduit des équations de consistance $f^{(n)}=\dot{f}^{(n)}=0$ (Eq. (56)).

D'après les relations (141), le durcissement par couche est purement cinématique linéaire et la restauration, indépendante de la couche considérée ( $A$ est une constante), peut être totale. Si la constance de $A$ est physiquement plausible, indépendance par rapport à la partie du matériau considéré des phénomènes de restauration essentiellement gérés par des mécanismes de diffusion, du moins pour $T / T_{\mathrm{F}} \geqq 0,3$, l'absence de seuil n'est pas conforme à l'expérience. Comme dans le modèle $\mathrm{LMAB}$, on suggère de réécrire les termes de restauration sous la forme :

$$
A\left(J_{2}\left(\underline{\mathbf{X}}^{\mathrm{p}(n)}\right)-J_{2}\left(\underline{\mathbf{X}}_{0}^{\mathrm{p}(n)}\right)\right)^{r} \frac{X_{i j}^{\mathrm{p}(n)}}{J_{2}\left(\underline{\mathbf{X}}^{\mathrm{p}(n)}\right)} .
$$

On remarque également que pour chaque couche, la variable isotrope est constante $\left(R_{0}^{\mathrm{p}(n)}\right)$. Ainsi, pour $\underline{\mathrm{X}}^{\mathrm{p}(n)}(0)=0$, durant un chargement cyclique, l'état stabilisé est atteint dès le premier cycle, ce qui est également contraire à l'expérience. Pour remédier à cette situation deux solutions sont possibles. En premier lieu, on admet qu'à l'instant zéro, les conditions initiales sur $\underline{\underline{X}}^{\mathrm{p}(n)}$ sont différentes de zéro à savoir négatives dans le cas d'une consolidation cyclique (comme pour le modèle LMX), ce qui produit effectivement une certaine progressivité dans l'établissement de la contrainte stabilisée. Cependant, cette solution semble physiquement déraisonnable puisque pour un premier chargement, le matériau devrait présenter systématiquement un écrouissage latent de sens inverse de celui de l'écrouissage. La seconde solution consiste à ne plus considérer les composantes isotropes $R^{\mathrm{p}(n)}$ comme des constantes mais de prendre, à l'exemple des modèles globaux, $\dot{R}^{\mathrm{p}(n)}$ fonction de la déformation cumulée (plastique ou visqueuse). Pour chaque sous-matériau, une cinétique du premier ordre telle que celle adoptée précédemment est tout à fait concevable, soit :

$$
\begin{aligned}
\dot{R}^{\mathrm{p}(n)}= & b^{(n)}\left(Q^{(n)}-R^{\mathrm{p}(n)}\right) \dot{p}, \\
& R_{(0)}^{\mathrm{p}(n)}=R_{0}^{\mathrm{p}(n)},
\end{aligned}
$$

et ainsi, chaque couche présenterait un durcissement cyclique. La contrainte locale $\underline{\mathbf{\sigma}}^{(n)}$ est donnée par l'équation de consistance, soit :

$$
\underline{\mathbf{\sigma}}^{(n)}=\underline{\mathbf{X}}^{\mathrm{p}(n)}+\frac{R^{\mathrm{p}(n)}}{\dot{R}^{\mathrm{p}(n)}}\left(\underline{\dot{\mathbf{g}}}^{(n)}-\underline{\mathbf{X}}^{\mathrm{p}(n)}\right),
$$

et la contrainte globale par :

$$
\underline{\mathbf{\sigma}}=\sum_{(n)} w^{(n)} \underline{\mathbf{\sigma}}^{(n)}
$$

En uniaxé, on a donc : $\underline{\mathbf{\sigma}}=\sum_{(n)} w^{(n)}\left(\underline{\mathbf{X}}^{\mathrm{p}(n)}+R^{\mathrm{p}(n)}\right)$ et la contrainte au cycle stabilisé pour une amplitude de déformation donnée $\left(\frac{\Delta \varepsilon_{1}^{\mathrm{p}}}{2}\right)$ correspondant à l'activation de $\left(n_{1}\right)$ couches, s'obtient par :

$$
\underline{\mathbf{\sigma}}=\sum_{1}^{\left(n_{1}\right)} w^{(n)}\left(\underline{\mathbf{X}}^{\mathrm{p}(n)}+Q^{(n)}\right) .
$$

Un net avantage de la structure multicouche par rapport aux modèles globaux provient de ce que les effets de mémoire de la prédéformation et d'évolution du module d'écrouissage tangent durant le cyclage, sont naturellement pris en compte. En effet, lorsqu'on augmente l'amplitude de la déformation de cyclage, on active de nouvelles couches, non sollicitées sous le plus faible niveau, ce qui entraîne une nouvelle phase de consolidation (ou inversement) jusqu'à ce que la nouvelle contrainte stabilisée soit atteinte, et ainsi de suite pour des amplitudes croissantes

Ainsi, dans ce type d'approche, il n'est pas nécessaire de définir une zone de non-écrouissage, c'est-à-dire d'hypersurface $G=0$ (Eq. (107)). Par contre, le choix du nombre de couches et l'identification de ce modèle n'est pas aisé car il existe, a priori, une infinité de solutions pour représenter ne seraitce qu'un essai de traction uniaxial. Dans le cas de l'acier 17-12 SPH, Blanchard [33] montre qu'une dizaine de couches est amplement suffisante pour décrire les essais classiques de fluage et de traction, et présente une technique d'identification dans le cas où tous les sous-matériaux ont les mêmes propriétés élastiques (modules $E, \nu$ ) et où les grandeurs isotropes sont des constantes. Par exemple, le fluage 
primaire est directement donné par la relation (143) :

$$
\frac{\mathrm{d} \varepsilon}{\mathrm{d} t}=A \frac{\sum_{(n)}\left(\frac{w^{(n)} X^{\mathrm{p}(n)^{r}}}{a^{(n)}} /\left(\frac{1}{E}+\frac{1}{a^{(n)}}\right)\right)}{\sum_{(n)} \frac{w^{(n)}}{1 / E+1 / a^{(n)}}},
$$

et la vitesse stationnaire par :

$$
\dot{\varepsilon}^{\text {st }}=A\left(\frac{\sigma-\sum_{(n)} w^{(n)} R_{0}^{p(n)}}{\sum_{(n)} w^{(n)} a^{(n) 1 / r}}\right) r
$$

En conclusion, moyennant les différentes modifications présentées, comportement viscoplastique quelle que soit la température considérée (Eq. (95a)), introduction de seuils sur les termes de restauration cinématique et en faisant dépendre les variables isotropes de la déformation cumulée $\dot{v}$, ce modèle est tout à fait apte à décrire correctement l'ensemble des observations expérimentales reportées au paragraphe 3 . On peut également remarquer que ce modèle présente une remarquable aptitude à décrire les effets de rochet 2D de tractiontorsion et particulièrement le phénomène d'adaptation observé pour les plus faibles températures [33, 34].

Ce modèle s'intègre parfaitement dans le moule thermodynamique évoqué en 4.2 .2 en choisissant simplement, dans le cas des relations (141), des potentiels de restauration $\Omega_{\mathrm{r}}^{(n)}$ tels que :

$$
\Omega_{\mathrm{r}}^{(n)}=\frac{A}{a^{\mathrm{p}(n)}(r+1)}\left(J_{2}\left(\underline{\mathbf{X}}^{\mathrm{p}(n)}\right)\right)^{r+1} .
$$

5.3.5 Le modèle multicouche série du LMX. - La version originale du modèle du LMX est purement plastique (Eq. (21)) et les lois d'évolution des variables internes cinématiques et isotropes sont données par les relations (29), soit par sous-matériau :

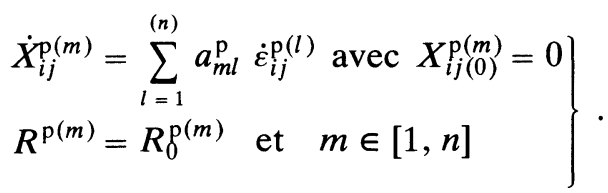

De plus, si toutes les couches sont élastiquement identiques, l'équation d'équilibre du système série s'écrit : $\underline{\varepsilon}^{\mathrm{p}}=\sum_{(n)} \underline{\boldsymbol{\varepsilon}}^{\mathrm{p}(n)}$ avec les équations de consistance $f^{(n)}=\dot{f}^{(n)}=0$ exprimant que $\underline{\mathbf{\sigma}}$ est toujours sur une surface $f^{(n)}$. Il est clair que sous cette forme dépouillée, ce modèle présente de nombreuses lacunes. Cependant, une version viscoplastique telle que celle présentée en (93a) est tout à fait envisageable :

$$
\dot{\varepsilon}_{i j}^{\mathrm{v}(n)}=\left\langle\frac{J_{2}\left(\underline{\mathbf{\sigma}}-\underline{\mathbf{X}}^{\mathrm{v}(n)}\right)-R_{0}^{\mathrm{v}(n)}}{K R_{0}^{\mathrm{v}(n)}}\right\rangle^{n^{*}(n)},
$$

avec $\underline{\mathbf{X}}^{\mathrm{v}(n)}$ et $R_{0}^{\mathrm{v}(n)}$ données par les relations (145). Moyennant des distributions assez larges du nombre de couches $(n)$ et des exposants $n^{*}$, cette modification devrait correctement intégrer les effets de viscosité, mais les phénomènes de restauration intrinsèquement liés au temps ne sont pas pris en compte.

Pour pallier cette carence, en suivant la démarche proposée pour le modèle parallèle, on préconise de réécrire la première relation (145) sous la forme :

$$
\begin{aligned}
\dot{X}_{i j}^{\mathrm{v}(m)}= & \sum_{l=1}^{(n)} a_{m l}\left(\dot{\varepsilon}_{i j}^{\mathrm{v}(l)}-A^{(l)} \times\right. \\
& \left.\times\left(J_{2}\left(\underline{\mathbf{X}}^{\mathrm{v}(l)}\right)-J_{2}\left(\underline{\mathbf{X}}_{0}^{\mathrm{v}(l)}\right)\right)^{r} \frac{X_{i j}^{\mathrm{v}(l)}}{J_{2}\left(\underline{\mathbf{X}}^{\mathrm{v}(l)}\right)}\right) .
\end{aligned}
$$

La proposition (147) montre que l'écoulement stationnaire est atteint lorsque toutes les composantes de la vitesse sont elles-mêmes dans un état stationnaire, soit :

$$
\dot{v}^{(l)}=A^{(l)}\left(J_{2}\left(\underline{\mathbf{X}}^{\mathrm{v}(l)}\right)-J_{2}\left(\underline{\mathbf{X}}_{0}^{\mathrm{v}(l)}\right)\right)^{r} .
$$

Dans sa version plastique, du point de vue cyclique, l'écrouissage cinématique étant une combinaison linéaire de forme linéaire et les variables isotropes étant constantes (Eq. (145)), ce modèle présente les mêmes caractéristiques que le modèle parallèle, à savoir que le premier cycle correspond au cycle stabilisé, que les courbes monotone et cyclique sont confondues (du moins avec $\underline{\mathbf{X}}_{(0)}^{(n)}=0$ ) et que les effets de mémoire de la préformation sont pris en compte. Pour remédier aux défauts, les deux solutions présentées pour le modèle parallèle restent valables. Dans sa version originale, les auteurs retiennent la première solution et considèrent que les valeurs initiales $\underline{\mathbf{X}}_{(0)}^{(n)}$ ne sont pas nulles, ce qui n'est pas physiquement acceptable pour les raisons que l'on a soulignées précédemment. A notre avis, la seconde solution qui consiste à prendre $R^{\mathrm{p}(n)}$ variable et fonction de $\dot{p}^{(n)}$ (ou $\left.\dot{v}^{\mathrm{p}(n)}\right)$, par exemple :

$\dot{R}^{\mathrm{p}(n)}=b^{(n)}\left(Q^{(n)}-R^{\mathrm{p}(n)}\right) \dot{p}^{(n)}$ avec $R_{(0)}^{\mathrm{p}(n)}=R_{0}^{\mathrm{p}(n)}$,

nous semble plus adaptée. La contrainte globale est accessible par l'équation de consistance $f^{(n)}=$ $\dot{f}^{(n)}=0$, d'où : $\underline{\mathbf{\sigma}}=\underline{\mathbf{X}}^{\mathrm{p}(n)}+\frac{R^{\mathrm{p}(n)}}{\dot{R}^{\mathrm{p}(n)}}\left(\dot{\underline{\mathbf{\sigma}}}-\underline{\dot{\mathbf{X}}}^{\mathrm{p}(n)}\right)$, qui, en uniaxé, redonne : $\sigma=X^{\mathrm{p}(n)}+R^{\mathrm{p}(n)}$, soit pour le cycle stabilisé correspondant à l'activation de $\left(n_{1}\right)$ couches, $\sigma=X^{\mathrm{p}\left(n_{1}\right)}+Q^{\left(n_{1}\right)}$. Dans cette approche, comme pour la précédente, il n'est pas nécessaire d'introduire le concept de surface de non-écrouissage, celui-ci étant naturellement présent dans la structure multicouche. Par contre, les problèmes d'identification (choix du nombre de couches et des 
paramètres affectés à chaque couche) demeurent difficiles à appréhender d'une manière rationnelle.

En conclusion, il semble tout à fait possible, moyennant les quelques modifications suggérées d'une façon non exhaustive, d'adapter ce modèle plastique aux conditions complexes de la viscoplasticité à haute température.

5.4 ConClusions. - Nous avons montré que les structures de l'ensemble des modèles à variables internes étudiés, avec quelques adaptations spécifiques aux conditions de la viscoplasticité à haute température, étaient aptes à décrire qualitativement, pour un isotherme donné, la plupart des phénomènes observés sous sollicitation uniaxiale (ou radiale). La différence essentielle entre les approches globale et multicouche réside dans le fait que les variables internes sont traitées respectivement, d'une manière continue faisant appel au concept de l'écrouissage cinématique non linéaire à mémoire évanescente pour appréhender correctement les conditions de retour en arrière, ou d'une manière discrète par l'intermédiaire de variables cinématiques linéaires ; le nombre de couches ainsi que leur activation liée aux équations de consistance permet de traduire les non-linéarités ainsi que les retours en arrière. Cependant, les modèles globaux, plus simples dans leur identification, doivent faire appel au concept de zone de non-écrouissage (surface $G$ ) pour tenir compte des effets de mémoire de la prédéformation, ce qui n'est pas le cas des modèles multicouches où cet effet est directement lié à la structure discrète. Pour intégrer la progressivité dans l'établissement de l'état stabilisé, il nous semble qu'à l'exemple des modèles globaux, les variables isotropes des modèles structure devraient dépendre de la déformation cumulée ( $\dot{v}$ ou $\left.\dot{v}^{(n)}\right)$. D'autre part, il est souhaitable que pour l'ensemble des modèles, il apparaisse des termes de restauration (avec seuils) portant sur les variables isotrope et cinématique, ce qui est le cas des modèles ONERA et LMAB et dans une moindre mesure dans les versions actuelles des modèles de l'EMP (variables isotropes) et du CEA (variables cinématiques).

\section{Sur quelques possibilités offertes par ces modèles.}

Pour juger de l'acuité de ces modèles à prédire qualitativement les phénomènes observés, il serait nécessaire, d'un commun accord entre les différents auteurs, d'élaborer une base unique de résultats expérimentaux sous différents types de sollicitation uniaxée (dans un premier temps) et relatif à un même matériau; par exemple le 17-12 SPH qui présente un comportement viscoplastique très marqué et pour lequel les résultats sont très nombreux parfois même contradictoires. Cette base pourrait comprendre, pour un isotherme déterminé : des essais de chargement monotone à différentes vitesses, des essais cycliques, avec et sans temps de maintien sous des niveaux de déformation croissants puis décroissants, des essais de fluage monotone et sous plusieurs niveaux de contrainte croissants et décroissants, des essais de relaxation avec différentes vitesses de montée en charge, des essais de vieillissement en différents points d'un cycle stabilisé et enfin toute la gamme des essais cycliques dans des conditions non symétriques; par exemple des essais de rochet 1D sous contrainte moyenne non nulle et des essais de relaxation de la contrainte moyenne lorsqu'on effectue des petits cycles après avoir préalablement cyclé le matériau sous un niveau de déformation plus élevé. Sur cette base communément admise, les auteurs devraient ensuite identifier leur propre modèle puisqu'il s'avère que les subtilités de l'identification liées aux multiples degrés de liberté dus à la complexité des formulations ne sont réellement maitrisées, du moins dans l'état actuel des « modes d'emplois », que par les concepteurs du modèle considéré. Dans l'optique actuelle des recherches, chaque école développe son propre modèle à l'aide, le plus souvent, de sa propre base expérimentale, ce qui rend toutes comparaisons quantitatives entre modèles très délicates. Ainsi, d'une façon non exhaustive et en fonction des documents en notre possession, on présente quelques simulations réalisées à l'aide de ces modèles identifiés essentiellement sur le 17-12 SPH. En effet, à notre connaissance, dans le cas de l'INCO 718, seul le modèle ONERA-LMT a été complètement identifié et dans une bien moindre mesure, le modèle LMAB, pour ce qui concerne exclusivement quelques aspects du fluage biaxé en traction-torsion.

Sur les figures 22,5 et 23 , on reporte quatre exemples de simulation d'essais de traction monotone réalisés à l'aide des modèles unifiés (ONERA, LMAB, Fig. 22), partitionné (EMP, Fig. 5) et multicouche parallèle (CEA, Fig. 23). Tous les modèles prévoient correctement ce type d'essai très simple et dans le dernier cas, on peut voir que dix couches sont suffisantes pour approximer la courbure et la continuité de la courbe expérimentale, du moins sur le faible domaine de déformation envisagée (Fig. 24).

En ce qui concerne le fluage, sur les figures 3,5 et 24 , on montre l'aptitude des modèles unifiés (LMAB, Fig. 3), partitionné (EMP, Fig. 5) et multicouche (CEA, Fig. 24) à prévoir les réponses à ce type de sollicitation et particulièrement la transition aiguë entre les déformations importantes liées au chargement et les faibles déformations relatives au fluage à long terme. De même, les déformations de fluage sous différents niveaux de contrainte (Fig. 3) sont assez bien décrites et l'on peut également voir sur cette figure, dans le cas spécifique du modèle 


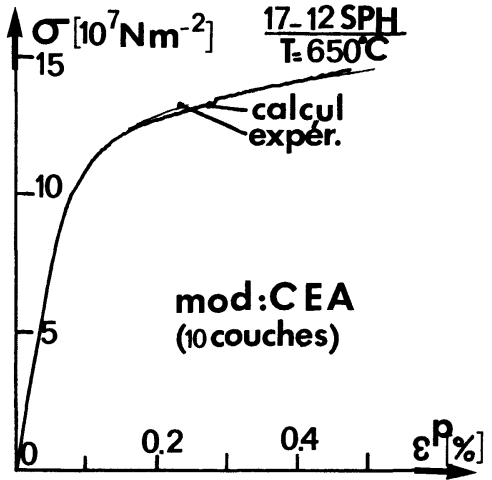

Fig. 23. - Exemple de modélisation d'une courbe effortdéformation à l'aide de l'approche multicouche du CEA ; acier 17-12 SPH à $650{ }^{\circ} \mathrm{C}$. (D'après Blanchard et Tortel [33], avec l'amabilité du $5^{\mathrm{e}}$ Post-SMIRT).

[An example of a stress-strain curve modelling from the multilayers approach of CEA $17-12 \mathrm{SPH}$ at $650^{\circ} \mathrm{C}$. (From Blanchard and Tortel [23], with courtesy of the 5th PostSMIRT).]

LMAB dans l'état de chargement passif, que les déformations de recouvrance sont également assez bien appréhendées. Cependant, dans le cas du modèle C.E.A. (Fig. 24), le fluage n'est imputable qu'au terme de restauration des variables cinématiques (Eq. (141)) et donc aux plus faibles températures où la restauration par diffusion est inefficace, cette approche ne prévoit pas de fluage (ni de relaxation), ce qui est contraire à l'expérience, d'où

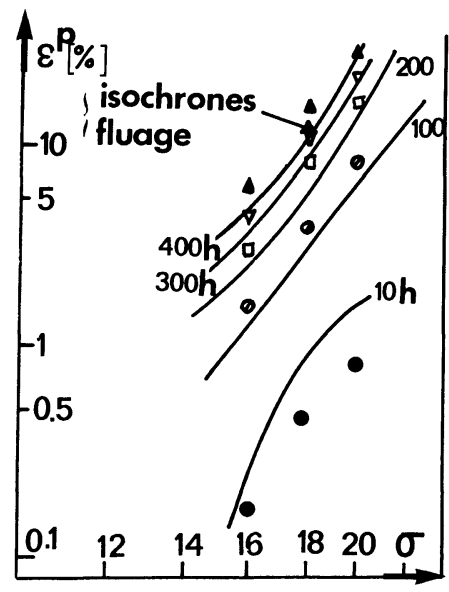

Fig. 24. - Modélisation des isochrones de fluage à $650^{\circ} \mathrm{C}$ d'un acier 17-12 SPH, avec la formulation multicouche du CEA. (D'après Blanchard et Tortel [33], avec l'amabilité du $5^{\mathrm{c}}$ Post-SMIRT).

[Simulation of the isochronic creep curves at $650{ }^{\circ} \mathrm{C}$ of a 17-12 SPH steel with the CEA model. (From Blanchard and Tortel [33], with courtesy of the 5th Post-SMIRT).] la nécessité de développer une version viscoplastique des modèles multicouches (Eq. (95)). Si dans leur ensemble, les modèles globaux sont capables de prévoir qualitativement la relaxation (de même avec une version viscoplastique des modèles multicouches), il s'avère que quantitativement, le passage fluage-relaxation n'est pas toujours optimisé, c'està-dire que les modèles sont correctement ajustés soit sur le fluage, soit sur la relaxation mais difficilement sur les deux à la fois. Ce cas de figure est caractéristique du modèle ONERA [17]. Par contre, ce type de difficulté a été particulièrement étudiée dans le cas du modèle partitionné de l'EMP et les auteurs montrent qu'il est parfaitement possible d'ajuster convenablement à la fois fluage et relaxation (Figs. 5 et 25). D'autre part, on peut signaler la très grande dispersion des résultats expérimentaux de relaxation liée à la difficulté de réaliser proprement ce type d'essai : seuls les essais effectués sur machine asservie, pilotée par rapport à une extensométrie directement fixée sur l'éprouvette, sont exploitables. De plus, une attention particulière doit être apportée à la vitesse de montée en charge précédant la relaxation [83].

L'illustration des possibilités des modèles unifiés ONERA-LMT Cachan et LMAB vis-à-vis des propriétés cycliques sont illustrées sur les figures 7,26 et

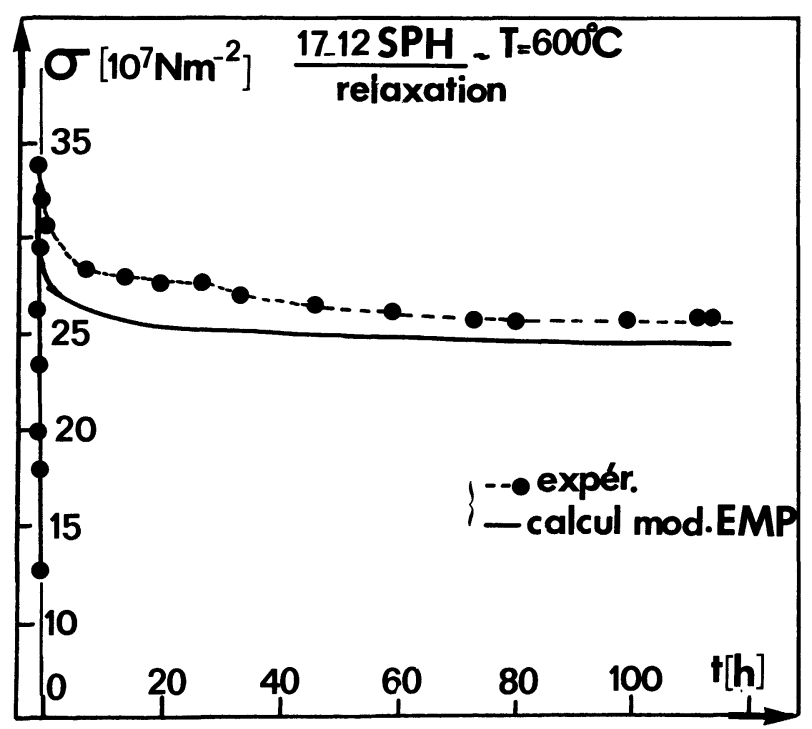

Fig. 25. - Acier inoxydable $17-12 \mathrm{SPH}$ à $600{ }^{\circ} \mathrm{C}$; modélisation d'un essai de relaxation, après mise en charge à vitesse imposée par le formalisme partitionné de l'E.M.P. (D'après Contesti [31], avec l'amabilité du GIS « Rupture à Chaud »).

[17-12 SPH stainless steel at $600{ }^{\circ} \mathrm{C}$; simulation of a relaxation test, after a loading at an imposed strain rate, E.M.P. partitionning model. (From Contesti [31], with courtesy of the GIS « Rupture à Chaud»).] 
27. Ainsi, on peut voir sur la figure 7 que la déconsolidation cyclique de l'INCO 718 est parfaitement pris en compte par le modèle ONERA, au même titre que la consolidation progressive de l'acier inoxydable pour qui une simulation à l'aide du modèle LMAB est reportée sur la figure 26.

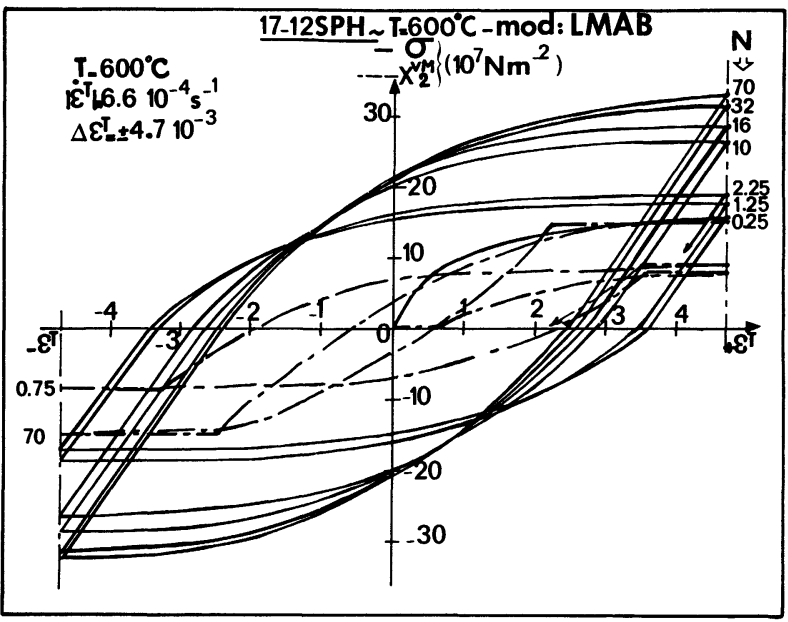

Fig. 26. - Acier inoxydable $17-12 \mathrm{SPH}$ à $600^{\circ} \mathrm{C}$; exemple de consolidation cyclique obtenue à l'aide du modèle unifié du LMAB. Evolution simultanée de $X^{\mathrm{vM}(2)}$. (D'après Delobelle et Oytana [77], avec l'amabilité du SMIRT 9).

[17-12 SPH stainless steel at $600{ }^{\circ} \mathrm{C}$; example of cyclic hardening predicted with the LMAB unified model. Simultaneous evolution of $X^{\mathrm{vM}(2)}$. (From Delobelle and Oytana [77], with courtesy of SMIRT 9).

Cette représentation montre clairement d'une part, l'évolution progressive du module tangent pour une déformation donnée, et d'autre part, l'apparition systématique de la déformation viscoplastique au voisinage de $\sigma_{z z}=0$, ces deux propriétés étant visibles sur les cycles expérimentaux des figures 6 et 9. La courbe de consolidation cyclique de l'acier 17$12 \mathrm{SPH}$ à $600{ }^{\circ} \mathrm{C}$, calculée avec ces deux modèles, est reportée sur la figure 27 . Globalement, la corrélation calcul-expérience est correcte. De même, sur la figure 28 réalisée à l'aide du modèle ONERA, on montre que l'activation de la consolidation cyclique pour des niveaux croissants de déformation est également restituée (rôle de la surface $G$ ). Pour ce qui est des autres modèles vis-à-vis des propriétés cycliques, hormis l'analyse théorique présentée précédemment, le manque de documents chiffrés en notre possession ne permet pas une analyse quantitative.

Les figures 11,29 et 30 montrent les possibilités offertes par les deux approches unifiées vis-à-vis des

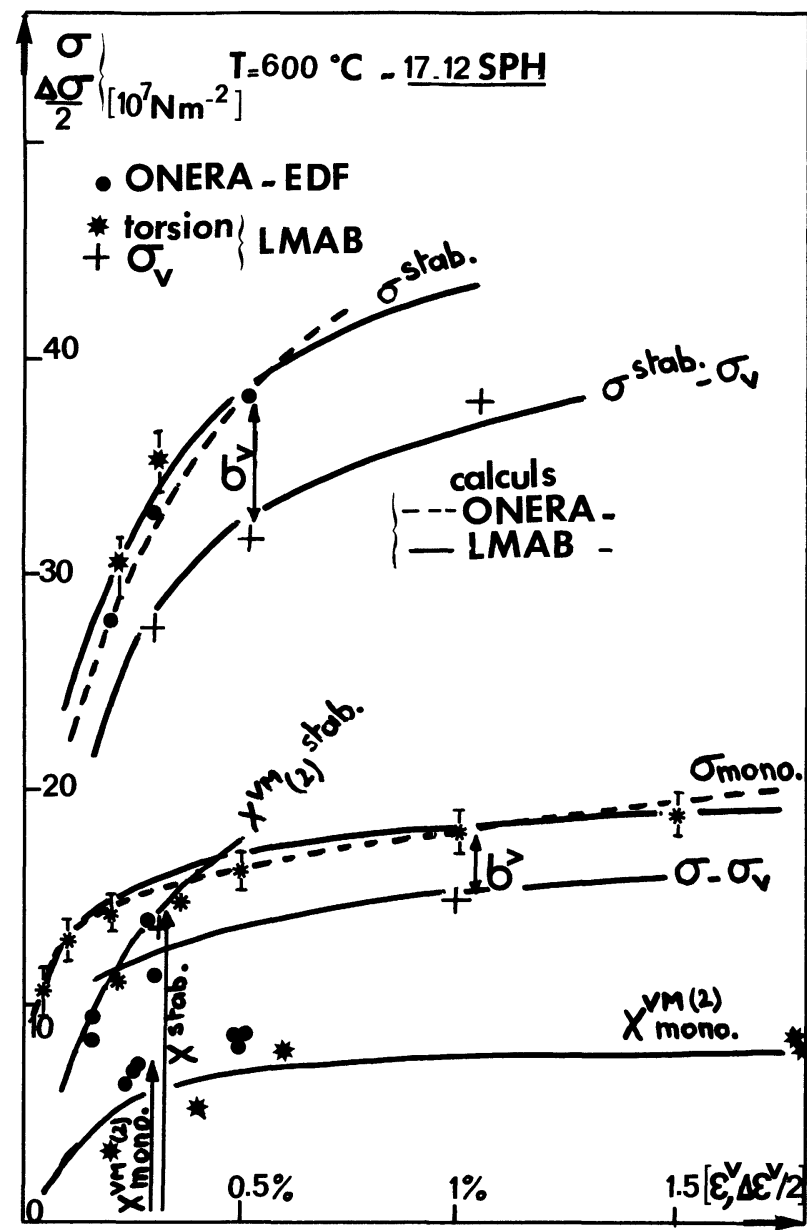

Fig. 27. - Acier inoxydable $17-12 \mathrm{SPH}$ à $600{ }^{\circ} \mathrm{C}$; prévisions des courbes monotone et cyclique à l'aide des deux modèles unifiés ONERA et LMAB. (D'après Nouailhas [17] et Delobelle, Oytana [77], avec l'amabilité de l'ONERA et du SMIRT 9).

[17-12 SPH stainless steel at $600{ }^{\circ} \mathrm{C}$; predictions of the monotonic and cyclic curves due to the two unified models of ONERA and LMAB. (From Nouailhas [17] and Delobelle, Oytana [77], with courtesy of ONERA and SMIRT 9).]

effets de restauration directement liés au temps. Les figures 11 (ONERA) et 29 (LMAB) reportent les prévisions théoriques de la chute de la contrainte d'écoulement, chiffrée par $\Delta \sigma_{r}$, en fonction du temps de maintien à contrainte nulle et de la déformation (ou de la contrainte pour des essais monotones, Fig. 29). Là encore, les prévisions de ces modèles sont satisfaisantes. Un autre effet du temps remarquablement bien rendu par le modèle ONERA est l'influence d'un temps de maintien au maximum de chaque cycle sur la courbe de consolidation cyclique (Fig. 12), ce dont on peut s'apercevoir sur la figure 30 donnant les prévisions de l'évolution de la contrainte au maximum du cycle 


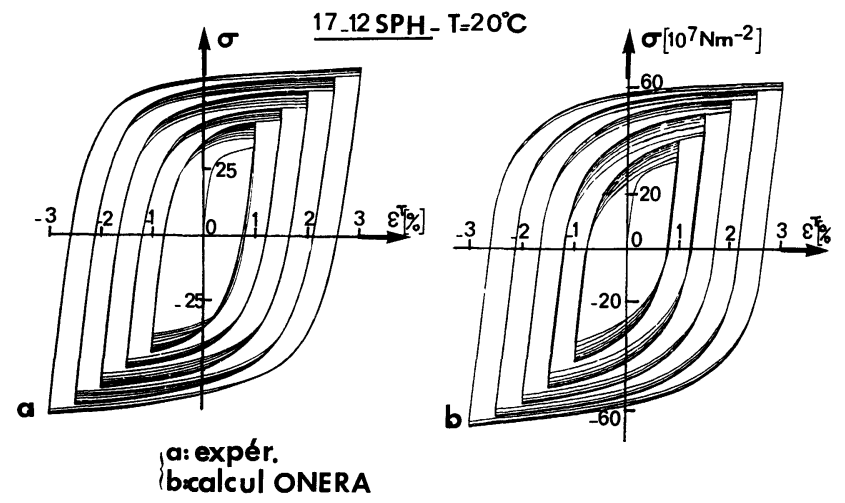

Fig. 28. - Acier inoxydable 316L à la température ambiante ; essais et simulation de la consolidation cyclique pour des niveaux croissants. Mise en évidence de l'activation de la consolidation pour chaque niveau. (D'après Chaboche, Dang-Van et Cordier [16], avec l'amabilité du SMIRT 5).

[316L stainless steel at room temperature; tests and simulations of cyclic hardening for increasing levels. The activation of hardening for each level is pointed out. (From Chaboche, Dang Van and Cordier [16], with courtesy of SMIRT 5).]

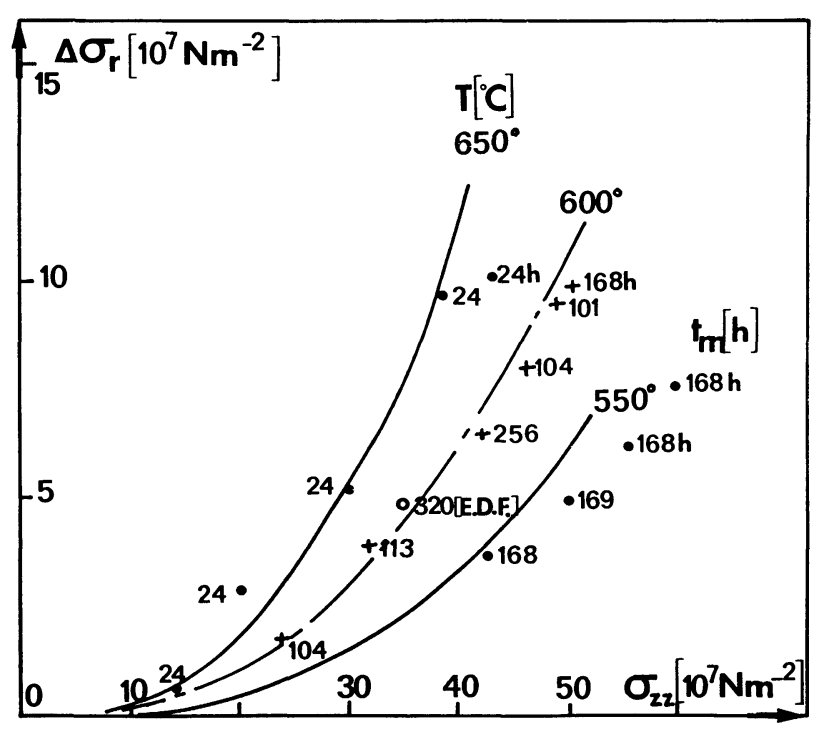

Fig. 29. - Acier 17-12 SPH, $550 \leqq T \leqq 650^{\circ} \mathrm{C}$; modélisation à l'aide du formalisme LMAB de l'amplitude maximale restaurable $\Delta \sigma_{\mathrm{r}}$ en fonction de la contrainte d'écoulement et pour trois isothermes. (D'après Delobelle et Oytana [9], avec l'amabilité du J. Nucl. Mater.).

[17-12 SPH, $550{ }^{\circ} \mathrm{C} \leqq T \leqq 650{ }^{\circ} \mathrm{C}$; evolution of the maximum recoverable magnitude $\Delta \sigma_{\mathrm{r}}$ versus the flow stress as predicted by the LMAB model, for three isotherm cases. (From Delobelle and Oytana [9], with courtesy of J. Nucl. Mater.).]

$\left(\sigma_{t \max }\right)$ et de la contrainte en fin de relaxation $\left(\sigma_{t \text { min }}\right)$ en fonction du temps de maintien $t_{\mathrm{m}}$. A l'aide de ces calculs, les auteurs montrent la nécessité d'avoir une composante de restauration sur les deux types de variables.

Il est bien évident que ces modèles sont plus ou moins aptes à décrire des effets plus complexes relatifs à des chargements cycliques dissymétriques tels que le rochet $1 \mathrm{D}$ ou la relaxation de la contrainte moyenne [77], mais leurs possibilités exactes ne peuvent être entièrement perçues qu'à l'aide de simulations numériques d'essais bien précis (qui d'ailleurs sont disponibles [84]), ce qui n'est pas réalisé pour la plupart des modèles.

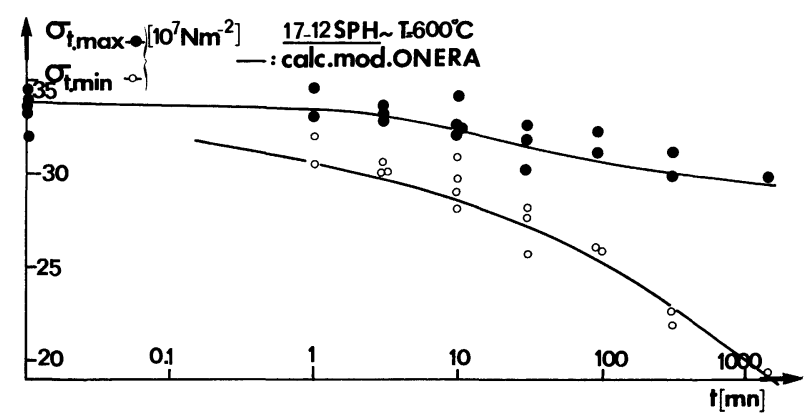

Fig. 30. - Acier 17-12 SPH à $600^{\circ} \mathrm{C}$. Prévision à l'aide du modèle ONERA de l'évolution de la contrainte au maximum du cycle stabilisé $\sigma_{t \text { max }}$ et de la contrainte en fin de relaxation $\sigma_{t \text { min }}$, en fonction du temps de maintien $t_{\mathrm{m}}$. (D'après Nouailhas [17], avec l'amabilité de l'ONERA).

[17-12 SPH steel at $600{ }^{\circ} \mathrm{C}$. Evolution of the maximum stress at the stabilized cycle $\sigma_{t \max }$ and of the stress at the end of the relaxation test $\sigma_{t \min }$ versus the holding time $t_{\mathrm{m}}$ as predicted by the ONERA model. (From Nouailhas [17], with courtesy of ONERA).]

En conclusion, l'ensemble des cinq modèles présentés, en intégrant pour certains d'entr'eux les modifications suggérées précédemment (particulièrement pour les multicouches) sont aptes à décrire qualitativement :

- le durcissement ou l'adoucissement cyclique,

- l'effet Bauschinger,

- les effets de mémoire de la déformation plastique,

- la transition aiguë plasticité-viscoplasticité,

- les phénomènes de viscosité (fluage, relaxation...),

- les effets de restauration de l'écrouissage à haute température sur l'ensemble des variables,

- les effets de vieillissement par le temps.

Actuellement, la confrontation de ces modèles, du moins à notre connaissance pour les modèles ONERA, LMAB et CEA, vis-à-vis des chargements cycliques dissymétriques à haute température est en cours d'étude. 
7. Développement actuel et futur de certains modèles vis-à-vis d'effets particuliers.

7.1 ETUDE 1D POUR UNE LARGE GAMME DE TEMPÉRATURE. - Les modèles sont souvent identifiés pour quelques isothermes, souvent proches des conditions d'utilisation industrielles des alliages considérés, mais rarement sur une plage étendue de température où des phénomènes particuliers, intrinsèquement liés à la nature et la microstructure de l'alliage, sont présents. Dans cette optique, on montre d'une part un exemple d'application du modèle ONERA-LMT à des conditions de chargement cyclique anisotherme sur le domaine de stabilité structurale de l'INCO 718 et d'autre part, les possibilités d'intégration, dans le formalisme général des modèles à variables internes, de quelques phéno. mènes d'instabilités structurales.

7.1.1 Condition de chargement anisotherme. L'exemple relaté, portant sur l'INCO 718, repose sur une extension du modèle ONERA-LMT Cachan (sous une forme très simplifiée) à des conditions de chargement anisothermes [85]. Les auteurs montrent qu'en choisissant judicieusement la fonction énergie libre et le pseudo-potentiel de dissipation, l'application de la double règle de normalité des matériaux standards généralisés (Eq. (9)) conduit aux équations anisothermes, soit : $\underline{\varepsilon}^{\mathrm{T}}=\underline{\boldsymbol{\varepsilon}}^{\mathrm{el}}+\underline{\boldsymbol{\varepsilon}}^{\mathrm{v}}$, où les déformations élastique et visqueuse sont données par :

$$
\left.\begin{array}{l}
\underline{\underline{\varepsilon}}^{\mathrm{el}}=[(1+\nu) / E(T)] \underline{\mathbf{\sigma}}-(\nu / E(T)) \operatorname{trace}[\underline{\mathbf{\sigma}}] \underline{\mathbf{I}}+\alpha^{*}(T)\left(T-T_{0}\right) \underline{\mathbf{I}} \\
\dot{\varepsilon}_{i j}^{\mathrm{v}}=\frac{2}{3} K^{*}(T)\left\langle J_{2}\left(\underline{\mathbf{\sigma}}-\underline{\mathbf{X}}^{\mathrm{v}}\right)-R^{\mathrm{v}}-R_{0}^{\mathrm{v}}(T)\right\rangle^{n^{*}} \cdot \frac{\sigma_{i j}^{\prime}-X_{i j}^{\mathrm{v}^{\prime}}}{J_{2}\left(\underline{\mathbf{\sigma}}-\underline{\mathbf{X}}^{\mathrm{v}}\right)} \quad \text { avec } \\
\dot{X}_{i j}=\frac{2}{3} a \dot{\varepsilon}_{i j}^{\mathrm{v}}-C X_{i j} \dot{v} \quad \text { et } \quad R^{\mathrm{v}}=Q_{1}(T)(1-\exp -b v)
\end{array}\right\} .
$$

On reconnaît aisément le modèle ONERA avec une équation d'état viscoplastique prise sous la forme d'une loi puissance, c'est-à-dire avec $\alpha_{0}=0$ et $K$ indépendant de $R^{\mathrm{v}}\left(K^{-n^{*}}=K^{*}(T)\right)$ dans l'équation (66a). De plus, il n'existe qu'une seule variable cinématique et une variable isotrope, toutes deux amputées des termes de restauration (Eq. (97c), (101) et (102)). Cette hypothèse n'est pas forcément justifiée compte tenu des domaines de températures et de contraintes élevés, envisagés. Les essais étant le plus souvent réalisés pour une amplitude de déformation cyclique fixée, les auteurs font également abstraction de la zone de non-écrouissage bien qu'elle puisse être affectée par les conditions de températures variables. Cependant, l'aspect novateur et positif est l'introduction de l'évolution continue avec la température des différents paramètres et grandeurs physiques du modèle, soit d'après le système (150) ; le module d'Young $E$, le coefficient de dilatation linéaire $\alpha^{*}$ et les paramètres $K^{*}$, $R_{0}^{\mathrm{v}}$ et $Q_{1}$. Le coefficient de Poisson $\nu$ et l'exposant $n$ * sont supposés constants. L'identification des paramètres du modèle en fonction de la température a été réalisée à l'aide d'essais statiques (mesure des constantes élastiques), de relaxation et d'adoucissement cyclique, pour différents isothermes et pour des amplitudes de déformation progressivement croissantes. Les auteurs montrent ainsi que la variable cinématique, déterminée par la perte de linéarité des boucles de traction-compression, n'est pas affectée par la température, l'essentiel des variations étant imputables à la variable isotrope $\left(Q_{1}, R_{0}^{\eta}\right)$, d'où la forme des équations (151). En lissant les valeurs expérimentales des différents paramètres, on peut écrire :

$$
\left.\begin{array}{rl}
E(T) & =e_{1}\left(1-\exp \left(e_{2} T\right)\right)+e_{3} \\
\alpha^{*}(T) & =\alpha_{1}^{*} T+\alpha_{2}^{*} \\
K^{*}(T) & =K_{0}^{*} \exp (-B T) \\
R_{0}^{\mathrm{v}}(T) & =k_{1}\left(1-\exp \left(-k_{2} T\right)\right)+k_{3} \\
Q_{1}(T) & =a_{1}\left(1-\exp \left(-a_{2} T\right)\right)+a_{3}
\end{array}\right\}
$$

$e_{1}, e_{2}, e_{3}, \alpha_{1}^{*}, \alpha_{2}^{*}, K_{0}^{*}, k_{1}, k_{2}, k_{3}, a_{1}, a_{2}$ et $a_{3}$ sont des constantes.

La vérification de ce modèle (Eqs. (150) et (151)) a exigé la réalisation d'essais thermomécaniques complexes, en particulier, d'essais cycliques en faisant varier, pour chaque essai, l'amplitude, la période et le déphasage entre les sollicitations thermique et mécanique [85]. Les calculs montrent que cette formulation est généralement en bon accord avec les résultats expérimentaux, aussi bien qualitativement que quantitativement [85]. Cependant, il a tout de même été trouvé que dans certains cas de chargement thermo-mécanique hors phase (1D), l'INCO 718 présente un durcissement cyclique, ce qui est en contradiction avec son comportement à température constante où il s'avère qu'il s'adoucit cycliquement indépendamment de la température. Le modèle dans son état actuel ne simule pas pareil effet et son analyse est en cours d'étude.

Comme nous l'avons évoqué précédemment, le modèle LMAB a été identifié, à l'aide d'essais 
isothermes dans le cas du 17-12 SPH, sur une plage de température sensiblement comprise entre 500 et $750{ }^{\circ} \mathrm{C}$. Bien que ces deux alliages sont très éloignés, la différence essentielle avec les équations (150) par rapport aux effets de la température est la présence de termes thermiquement activés $\left(\exp -\frac{\Delta H}{k T}\right.$, Eqs. (71a), (74), (130) et (131)) aussi bien dans l'équation d'état que sur les variables internes. L'exposant $n^{*}$ est également une fonction décroissante de la température. D'autre part, $R^{\mathrm{v}}$ étant fonction de la température, la variable $\underline{x}^{\mathrm{vM}(2)}$ l'est également puisque $R^{\mathrm{v}}$ conditionne l'évolution asymptotique de cette variable. L'extension du domaine de validité de ce modèle jusqu'à la température ambiante est en cours d'étude et se heurte, comme nous le verrons ci-dessous, au problème général des températures intermédiaires dans les solutions solides où les interactions dislocations-défauts ponctuels sont efficaces. Ultérieurement, lorsque ce problème sera correctement résolu, la confrontation de cette formulation à des conditions anisothermes est tout à fait souhaitable.

7.1.2 Exemple de prise en compte de l'évolution structurale consécutive à des traitements thermiques. On présente succinctement dans le cas du superalliage INCO 100, un exemple de prise en compte par un modèle à variable interne de l'effet de l'évolution structurale liée à des traitements thermiques préalables de surchauffe, sur les propriétés cycliques à 900 et $1000^{\circ} \mathrm{C}$ [86]. Sur cet alliage durci par précipitation structurale de $\gamma^{\prime}$, si l'on réalise, à partir de l'état de référence correspondant à un maintien de $4 \mathrm{~h}$ à $900^{\circ} \mathrm{C}$, une brève surchauffe $(10 \mathrm{~min})$ jusqu'à des températures comprises entre 1000 et $1100^{\circ} \mathrm{C}$, la structure obtenue après trempe révèle clairement la redissolution des petits précipités, d'autant plus rapidement que la surchauffe est élevée, seuls les gros subsistent. Si après ce même type de traitement, on réalise un palier de $10 \mathrm{~mm}$ à $900{ }^{\circ} \mathrm{C}$, on observe après trempe une nouvelle génération de très fins précipités de $\gamma^{\prime}$. Il est bien évident que les propriétés cycliques sont sensibles à l'état de la microstructure et les auteurs montrent, pour un isotherme fixé à $900{ }^{\circ} \mathrm{C}$ par exemple, que la disparition momentanée des plus fins précipités entraîne un net durcissement cyclique, évanescent avec le temps, au fur et à mesure que la nouvelle microstructure, correspondant à l'isotherme considéré, se rétablisse [86]. De façon à intégrer ces nouveaux phénomènes au modèle viscoplastique de l'ONERA (sous une forme simplifiée) les auteurs proposent l'introduction de deux nouvelles variables internes, à savoir, une température fictive $T_{\mathrm{f}}$ qui représente l'évolution de la fraction volumique des précipités $\gamma^{\prime}$ et une nouvelle variable isotrope $\mathbb{R}^{*}$ qui représente les variations de rayon de ces mêmes précipités. Ainsi, en $1 \mathrm{D}$, on écrit :

$$
\left.\begin{array}{l}
\dot{\varepsilon}^{\mathrm{v}}=\left\langle\frac{\sigma-X^{\mathrm{v}}-R^{\mathrm{v}}-\mathbb{R}^{*}}{K}\right\rangle^{n^{*}} \operatorname{sign}\left(\sigma-X^{\mathrm{v}}\right) \\
\dot{X}^{\mathrm{v}}=a \dot{\varepsilon}^{\mathrm{v}}-C X^{\mathrm{v}}\left|\dot{\varepsilon}^{\mathrm{v}}\right| \text { avec } X^{\mathrm{v}}(0)=0 \\
R^{\mathrm{v}}=Q_{1}^{\mathrm{v}} \\
\dot{T}_{\mathrm{f}}=\eta^{*} \frac{T-T_{\mathrm{f}}}{\alpha^{*}} \text { et } \dot{\mathbb{R}}^{*}=-\frac{\mathbb{R}^{*}}{\beta^{*}}+\eta^{*} a^{*}\left\langle T_{\mathrm{f}}-T\right\rangle
\end{array}\right\}
$$

$T$ est la température actuelle, $\alpha^{*}, \beta^{*}$ et $a^{*}$ sont des coefficients pouvant être fonction de la température et $\langle\cdot\rangle$ a sa signification habituelle. Le paramètre $\eta^{*}$ est tel que $\eta^{*}=0$ si $0<T_{\mathrm{f}}-T<b^{*} \mathbb{R}^{*}$, $b^{*}$ étant un nouveau coefficient dépendant de la température.

Plusieurs remarques s'imposent. Il n'existe qu'une seule variable cinématique amputée de son terme de restauration, ce qui peut être restrictif puisque les températures de référence sont très élevées. D'autre part, l'étude portant sur l'état stabilisé, la variable isotrope $R^{v}$ est prise dans son état asymptotique $Q_{1}^{v}$. Puisque la nouvelle variable isotrope métallurgique $\mathbb{R}^{*}$ est introduite dans l'équation d'état sous une forme additive, elle se trouve déconnectée de la variable isotrope mécanique $R^{v}$ (fonction de la déformation cumulée) et si cette hypothèse est justifiée pour l'étude de l'état stabilisé, elle peut être discutable dans le cas des régimes cycliques transitoires après surchauffe, où une forme multiplicative serait peut-être plus appropriée.

Le premier terme de la cinétique de $\mathbb{R}^{*}$ traduit la diminution de la variable isotrope globale $\left(R^{v}+\mathbb{R}^{*}\right)$ due à la croissance des précipités alors que le second, n'a aucune influence si $\left(T_{\mathrm{f}}-T\right)<0$ (période de surchauffe) ou $0<T_{\mathrm{f}}-T<b^{*} \mathbb{R}^{*}$ (cas intermédiaire), mais par contre, fait croître $\left(R^{v}+\mathbb{R}^{*}\right)$ lorsque $T_{\mathrm{f}}-T>b^{*} \mathbb{R}^{*}$ (période de refroidissement). Dans ce dernier cas, $\mathbb{R}^{*}$ passe rapidement par un maximum en fonction du temps, la partie croissante correspondant au durcissement cyclique obtenu après refroidissement et la partie décroissante a son évanescence liée à la croissance des précipités. Globalement, cette approche prévoit bien les effets de microstructure sur l'état cyclique stabilisé de l'IN 100 [86].

Un autre effet microstructural rencontré dans le 17-12 SPH, pour des températures comprises entre 550 et $750{ }^{\circ} \mathrm{C}$, est la précipitation hétérogène sur les joints de grains des carbures $\mathrm{M}_{23} \mathrm{C}_{6}$ [87]. Cette précipitation continue affecte les propriétés mécaniques et en particulier, une étude détaillée de l'évolution de la vitesse de fluage en fonction du temps à partir de l'état hypertrempé, révèle clairement la présence d'un maximum (oscillation) que l'on corrèle assez bien avec le diagramme de précipitation hétérogène de $\mathrm{M}_{23} \mathrm{C}_{6}[87,88]$. L'intégration de ce type d'observation dans le modèle LMAB est en cours de réalisation et l'on se propose d'introduire, sous une 
forme multiplicative, à la variable scalaire $R^{\mathrm{v}}$ une fonction décroissante simple de la fraction précipitée. Dans le cadre des théories classiques de la précipitation par diffusion à longue distance, on écrira la fraction précipitée sous la forme $C_{(t)}^{*} / C_{(\infty)}^{*}=\left(1-\exp -(t / \tau)^{n *}\right)$. A l'aide de ce formalisme, on pense effectivement restituer les différentes formes observées des courbes de fluage.

7.1.3 Cas des températures intermédiaires dans les solutions solides. - Les solutions solides C.C., C.F.C. ou H.C. sollicitées dans le domaine des températures intermédiaires $\left(0,2 \leqq T / T_{\mathrm{F}} \leqq 0,3\right)$ présentent fréquemment des comportements inverses de ceux rencontrés aux basses et hautes températures (voir ci-dessous) ainsi que de nombreux phénomènes d'instabilités, physiquement bien compris et généralement attribués aux interactions de configuration, à courte ou moyenne distance, entre dislocations et défauts ponctuels [89 à 91], mais phénoménologiquement très mal modélisés ou tout simplement ignorés.

Par exemple, dans le cas du 17-12 SPH, les courbes de traction réalisées entre 200 et $550^{\circ} \mathrm{C}$, pour des vitesses comprises entre $10^{-4}$ et $10^{-2} \mathrm{~s}^{-1}$, présentent une succession de décrochements caractéristiques de l'effet Portevin-Le Chatelier imputable aux interactions dislocations-amas de défauts ponctuels dans les C.F.C. [92], et l'analyse des contraintes d'écoulement, pour une déformation donnée, en fonction de la température montre clairement l'apparition d'un maximum dont la position est sensible à la vitesse de déformation (Fig. 31) [46, 47]. On peut mentionner que de telles instabilités sont également

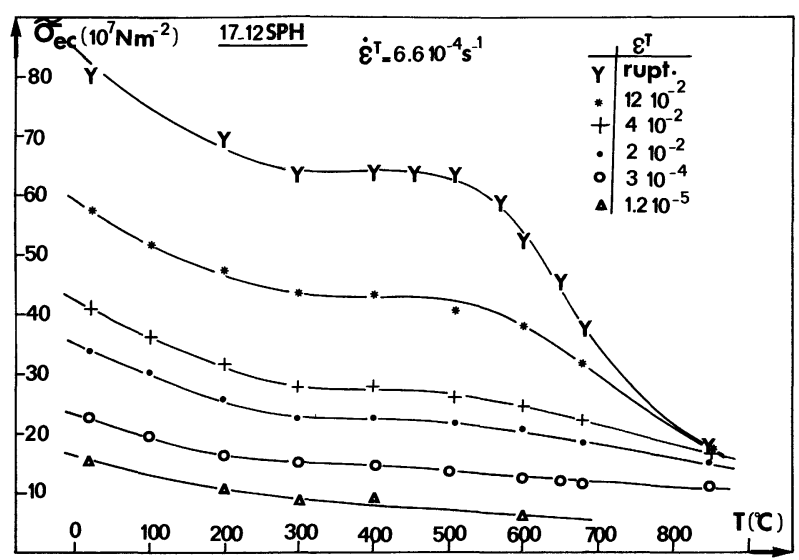

Fig. 31. - Acier 17-12 SPH ; évolution de la contrainte d'écoulement, pour différentes déformations, en fonction de la température. Mise en évidence du plateau entre 300 et $500{ }^{\circ} \mathrm{C}$, imputable aux interactions dislocations-défauts ponctuels. (D'après Delobelle [47]).

[17-12 SPH ; evolution of the flow stress for various strains versus temperature. The occurrence of a plateau between $300{ }^{\circ} \mathrm{C}$ and $500{ }^{\circ} \mathrm{C}$, due to points defects-dislocations interactions is pointed out. (From Delobelle [47]). présentes dans les essais à $\dot{\sigma}$ imposée [83]. Simultanément, on assiste à la disparition progressive des phénomènes visqueux, le microfluage remplace le fluage thermiquement activé et l'on observe également le développement d'une phase de consolidation cyclique maximale [47, 93]. Cette tendance est illustrée sur la figure 32, où l'on reporte l'évolution du rapport entre la composante visqueuse (déterminée par la méthode de la relaxation inverse) et la contrainte d'écoulement, pour des déformations fixées, en fonction de la température.

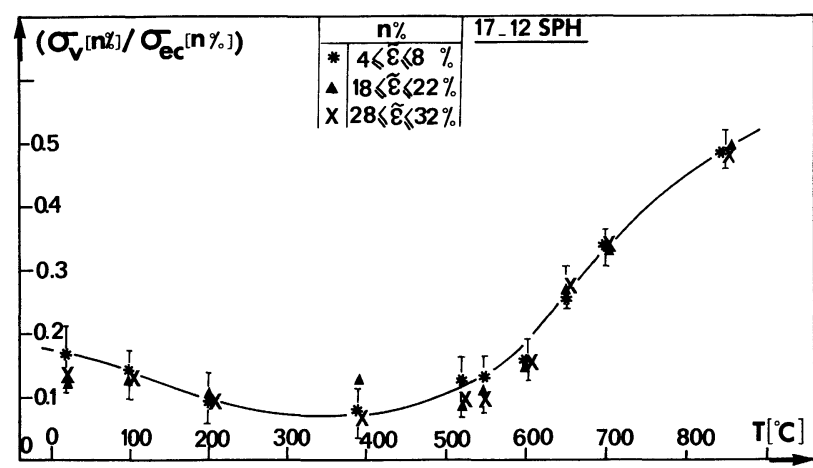

Fig. 32. - Acier 17-12 SPH ; mise en évidence du minimum sur la composante visqueuse $\sigma_{\mathrm{v}}$ lorsqu'apparaît un maximum sur la contrainte d'écoulement (Fig. 31). (D'après Delobelle [47]).

[17-12 SPH stainless steel ; evidence of a minimum of the viscous component $\sigma_{v}$ (Fig. 31). (From Delobelle [47]).]

On constate qu'au maximum de la contrainte d'écoulement (Fig. 31) correspond un minimum sur la composante visqueuse (Fig. 32), c'est-à-dire que lorsque les interactions sont maximales, le comportement de cet acier devient essentiellement plastique. D'autre part, le coefficient $n^{*}$, de sensibilité de la vitesse de déformation à la contrainte, devient négatif, en d'autres termes, plus la vitesse de déformation est élevée, plus la contrainte d'écoulement est faible [47]. Cette anomalie est caractéristique de la zone d'apparition du phénomène Portevin-Le Chatelier qui, compte tenu de la plage de température considérée où les phénomènes de diffusion à longue distance sont très lents, ne peut être imputable qu'aux interactions à courte distance du type Snoek-Schoek $[89,90]$. Cette interaction peut être caractérisée à l'aide de mesures de retour de limite d'élasticité qui consistent à chiffrer l'accroissement du seuil d'écoulement $\Delta \sigma_{\mathrm{s}}$ après vieillissement sous contrainte. Cette technique s'est révélée particulièrement fructueuse dans le cas des solutions solides hexagonales [94, 95] et cubiques centrées [96, 97].

Dans l'exemple du 17-12 SPH (C.F.C.), on montre que $\Delta \sigma_{\mathrm{s}}$ passe par un maximum lorsque simultanément, la contrainte d'écoulement présente un maxi- 
mum et la composante visqueuse un minimum. Ainsi, les différentes manifestations de ce type d'interaction configurationnelle peuvent être quantitativement analysables à l'aide de l'étude de l'évolution de $\Delta \sigma_{\mathrm{s}}$ en fonction des paramètres physiques maîtrisables $\left(\varepsilon, \sigma, t_{\mathrm{v}}, T\right)$. Cette analyse est trop complexe pour être exposée en détail, mais nous évoquerons les éléments essentiels ainsi que les possibilités d'intégration dans le contexte du modèle LMAB [47]. Puisque $\Delta \sigma_{\mathrm{s}}$ est positif et évanescent avec la déformation, contrairement aux régimes des basses et hautes températures, le matériau présente un écrouissage par le temps et une restauration par la déformation [47, 98]. Ainsi, en régime dynamique, les phénomènes d'instabilités pourraient résulter de l'interaction entre des termes simultanément présents mais antagonistes tels que les écrouissages par la déformation ou par le temps. En dehors des températures intermédiaires, les deux contributions ont des vitesses trop éloignées pour qu'une interaction soit possible, d'où le retour à un comportement classique.

Dans ce contexte, on attribue à chaque type d'interaction $(i)$ dislocations-défauts ponctuels en configuration, i-i, i-s ou s-i-s [99] (i ; interstitiels, s ; substitutionnels), une nouvelle variable interne $R^{*(i)}$, dont la cinétique s'écrit en vertu des concepts physiques de l'interaction [92], sous la forme :

$$
\dot{R}^{*(i)}=-p^{(i)} R^{*(i)}|\dot{\varepsilon}|+\frac{R_{(\infty)}^{(i)}(T)-R^{*(i)}}{\tau_{\mathrm{SN}}^{(i)}} .
$$

Dans cette relation, $p^{(i)}$ est une constante, $\tau_{\mathrm{SN}}^{(i)}$ le temps de relaxation intrinsèque à l'interaction considérée et $R^{*(i)}(T)$ sa fonction d'efficacité dépendant de la température. $\tau_{\mathrm{SN}}^{(i)}$ est thermiquement activé et $R^{*(i)}(T)$ correspond à une répartition gaussienne, soit :

$$
\tau_{\mathrm{SN}}^{(i)}=\tau_{\mathrm{SN}}^{(i)}(0) \exp \frac{W^{(i)}}{k T}
$$

et

$$
R^{*(i)}(T)=R_{(\infty)}^{*(i) \text { sat }} \exp -\left(\frac{T-T_{\mathrm{i}}}{A_{\mathrm{i}}}\right)^{2},
$$

$\tau_{\mathrm{SN}(0)}^{(i)}, W^{(i)}, R_{(\infty)}^{*(i) \text { sat }}, T_{\mathrm{i}}$ et $A_{\mathrm{i}}$ sont des constantes caractérisant l'interaction. Le premier terme de (153) correspond bien à une restauration par la déformation et le second à un écrouissage par le temps. De plus, pour une vitesse $\dot{\varepsilon}_{\mathrm{t}}$ fixée, la contribution stationnaire de la composante $(i)$, à la contrainte d'écoulement est donnée par :

$$
R^{*(i) \mathrm{st}}=\frac{R_{(\infty)}^{*(i)}(T)}{1+\tau_{\mathrm{SN}}^{(i)} p^{(i)}\left|\dot{\varepsilon}_{\mathrm{t}}\right|},
$$

qui est une fonction décroissante de la vitesse, d'où une possible explication de la valeur négative de l'exposant $n^{*}$ (effet de viscosité inverse !). A l'aide d'une étude détaillée des cinétiques $\Delta \sigma_{\mathrm{s}}=f\left(t_{\mathrm{v}}, T\right)$, on dénombre sensiblement cinq interactions, d'où $(i)=5$ [47]. A l'exemple des deux paragraphes précédents, les variables $R^{*(i)}$ peuvent être combinées à la variable isotrope $R^{v}$ sous des formes additives ou multiplicatives. Compte tenu que $\Delta \sigma_{\mathrm{s}}$ est étroitement fonction de la déformation, on adopte la seconde possibilité et l'on définit la nouvelle fonction isotrope $\mathcal{R}^{* v}$ par :

$$
\mathcal{R}^{* v}=R^{\mathrm{v}}\left(1+\gamma^{*} \frac{\sum_{i=1}^{5} R^{*(i)}}{\sum_{i=1}^{5} R^{*(i) \text { sat }}}\right) \text {. }
$$

$\gamma^{*}$ est une constante et ainsi, lorsque $\dot{\varepsilon}_{\mathrm{t}} \rightarrow \infty$, $\mathcal{R}^{* v}=R^{v}$ et il en est de même lorsque les différentes contributions sont négligeables $\left(\sum_{(i)} R^{*(i)} \simeq 0\right)$, mais lorsque $\dot{\varepsilon}_{\mathrm{t}} \rightarrow 0, \mathcal{R}^{* \mathrm{v}}$ est maximum soit :

$$
\mathcal{R}^{* \mathrm{v}}=R^{\mathrm{v}}\left(1+\gamma^{*} \frac{\sum_{i=1}^{5} R_{(\infty)}^{*(i)}}{\sum_{i=1}^{5} R_{(\infty)}^{*(i) \text { sat }}}\right) .
$$

Ces conclusions sont qualitativement en phase avec l'expérience. Cependant, la composante visqueuse $\sigma_{v}$ donnée par les équations (75) et (77), doit passer par un minimum lorsque $\mathcal{R}^{* v}$ atteint son maximum, condition qui peut être atteinte en écrivant $\alpha_{\mathrm{K}}(T)$ (Eq. (75)) sous la forme :

$$
\alpha_{\mathrm{K}}(T)=\alpha_{\mathrm{K}}(0)\left(1-\gamma^{*} \frac{\sum_{(i)} R_{(\infty)}^{*(i)}}{\sum_{(i)} R_{(\infty)}^{(i) \text { sat }}}\right)
$$

$\sigma_{v}$ étant accessible par la relation (77).

En conclusion, on vient de montrer que les différentes manifestations souvent complexes des interactions à courtes distances entre dislocations et défauts ponctuels dans les solutions solides, peuvent être, au moins qualitativement, intégrées dans un modèle unifié à variables internes et une solution réside dans le formalisme des équations (153) à (158). Quantitativement, l'extension de l'approche LMAB à cette plage de température est en voie d'achèvement et ce modèle sera donc identifié et utilisable entre 20 et $750{ }^{\circ} \mathrm{C}$.

On peut remarquer que si sur le principe, la démarche adoptée est voisine de celle de Miller et coll. [46, 100], qui d'ailleurs s'insère dans un certain consensus quant à la nature physique des phénomènes observés [90-92, 101], la formulation proposée est plus conséquente puisqu'elle est capable de restituer des phénomènes transitoires tels que les 
crochets de retour de limite d'élasticité en fonction de la température et de la déformation.

7.1.4 Conclusions. - D'une manière non exhaustive, on vient de montrer qu'il était possible, par le biais des modèles à variables internes et moyennant l'introduction de nouvelles variables isotropes $R^{*}$, plus ou moins couplées à $R^{\mathrm{v}}$, de décrire quelques phénomènes microstructuraux souvent liés aux conditions anisothermes. En fait, chaque nouvelle variable $R^{*}$ est directement connectée à un processus physique bien identifié, ce qui facilite l'identification de sa cinétique, mais la principale difficulté réside dans la compréhension et la modélisation de l'interaction de cette nouvelle variable sur l'ensemble des autres variables. Il s'avère généralement, qu'expérimentalement, chaque nouvelle variable influence l'ensemble des autres, ce qui conduit à des modélisations souvent complexes (Eqs. (153) à (158) par exemple).

\subsection{ETUDE DES CHARGEMENTS BIDIMENSIONNELS.}

Avec l'avènement des nouvelles machines hydrauliques biaxées (traction-torsion) pouvant éventuellement être pilotées par micro-ordinateur, le vaste champ d'étude du domaine des chargements 2D a pris un très net regain d'intérêt durant ces dernières années. Les trois axes principaux abordés traitent de l'étude des surfaces de charge ou des surfaces équipotentielles, du comportement cyclique en condition de chargement hors phase et enfin de l'étude du rochet de traction-torsion. Toujours dans l'optique des modèles à variables internes, nous évoquons succinctement les développements récents de ces trois aspects fondamentaux.

7.2.1 Les surfaces de charge. - Comme nous l'avons mentionné précédemment à plusieurs reprises, l'hypothèse restrictive fondamentale des cinq modèles présentés est l'absence de distorsion avec la déformation des surfaces $f$ ou $\Omega$. Expérimentalement (Fig. 33), il s'avère qu'avec la déformation, ces surfaces présentent un effet global de rotation combiné à un effet de pointe, plus ou moins marqué, dans la direction du chargement et un aplatissement conséquent dans la direction opposée [22-26, 102, 103]. Cette distorsion est certainement liée au déclenchement des différents systèmes de glissement lorsque la contrainte seuil résolue sur chaque type de plan est atteinte. On présente plusieurs tentatives de modélisation phénoménologique de ces effets.

Dans la formulation de Baltov et Sawczuk [24], on introduit dans la description des surfaces de charge un opérateur du $4^{\mathrm{c}}$ ordre $M_{i j k l}$ (par exemple, Eq. (160)). Contrairement à l'approche d'Edelman et Drucker [104] où aucune hypothèse n'est formulée, quant à la forme de cet opérateur, les auteurs proposent d'écrire $M_{i j k l}$ sous la forme d'une combinaison linéaire des cas isotrope et anisotrope.

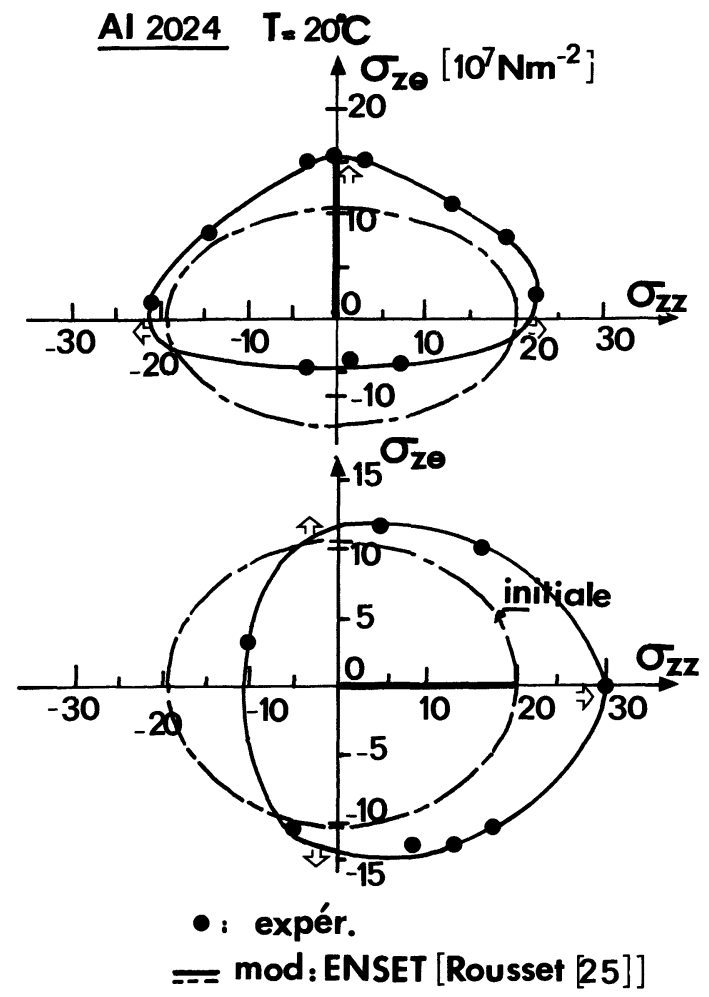

Fig. 33. - Mise en évidence des déformations des surfaces de charge (effet de pointe et d'aplatissement), ainsi que l'effet croisé. Cas de l'aluminium 2024 écroui en traction jusqu'à $\varepsilon^{\mathrm{p}}=1,5 \%$ puis retour à $\varepsilon^{\mathrm{p}}=0$. (D'après Rousset [25] Thèse, France).

[Distorsion of the yield surface on 2024 aluminium alloy. Monotonic tensile test up to $\varepsilon^{\mathrm{p}}=1.5 \%$ and reverse distorsion in subsequent compression up to $\varepsilon^{p}=0$. (From Rousset [25], Thesis, France).]

La partie anisotrope ne dépend que des déformations $\varepsilon_{i j}^{\mathrm{p}}$ et puisque la surface se translate cinématiquement, dans le cas particulier d'un écrouissage linéaire : $\left(X_{i j}^{\mathrm{p}}=a \varepsilon_{i j}^{\mathrm{p}}\right)$, l'expression de la surface seuil s'écrit :

$$
\begin{aligned}
f=\left\{\frac{3}{2}\left(I_{i j k l}+A^{+} X_{i j}^{\mathrm{p}^{\prime}} X_{k l}^{\mathrm{p}^{\prime}}\right) \times\right. \\
\left.\quad \times\left(\sigma_{i j}^{\prime}-X_{i j}^{\mathrm{p}^{\prime}}\right)\left(\sigma_{k l}^{\prime}-X_{k l}^{\mathrm{p}^{\prime}}\right)\right\}^{1 / 2}-R_{0}^{\mathrm{p}}
\end{aligned}
$$

avec $I_{i j k l}$ tenseur unité, et $A^{+}$une constante.

Dans le plan du déviateur des contraintes, l'équation (159) apporte une déformation symétrique de la surface $f$, c'est-à-dire que $f$ conserve une forme elliptique mais avec une possibilité de rotation des axes de symétrie.

Cependant, ce modèle ne prévoit pas de distorsion conséquente et en aucun cas d'effet croisé (Fig. 33), c'est-à-dire de déformation latérale du critère (dans la direction perpendiculaire au trajet de chargement). Ce dernier point, très important dans le cas des chargements hors phase, est en totale contradiction avec les observations expérimentales réalisées 
sur de nombreux alliages [25, 102-106] et a conduit les équipes ONERA-LMT [107] à modifier la surface seuil $f$ (Eq. (159)) sous la forme :

$$
\begin{aligned}
f=\left(\frac{3}{2} \frac{M_{i j k l}}{\left(1+\frac{3}{2} A_{0}^{+} p^{2}\right)} \cdot\left(\sigma_{i j}^{\prime}-X_{i j}^{\mathrm{p}^{\prime}}\right) \times\right. \\
\left.\times\left(\sigma_{k l}^{\prime}-X_{k l}^{\mathrm{p}^{\prime}}\right)\right)^{1 / 2}-R_{0}^{\mathrm{p}}-R^{\mathrm{p}} .
\end{aligned}
$$

L'écriture de $M_{i j k l}$ est identique à celle de Baltov et Sawczuk. L'introduction du dénominateur $\left(1+\frac{3}{2} A_{0}^{+} p^{2}\right)$ conduit à une expansion latérale de cette surface, ce dont on peut facilement s'apercevoir dans le cas des essais uniaxés de traction ou de torsion pure (seuls cas où la rotation de l'ellipse est nulle) et où les demi-axes de l'ellipse s'expriment par:

$$
\left.\begin{array}{rl}
a_{0}= & R_{0}^{\mathrm{p}}+R^{\mathrm{p}} \\
b_{0}= & \left(R_{0}^{\mathrm{p}}+R^{\mathrm{p}}\right)\left(1+\frac{3}{2} A_{0}^{+} \varepsilon_{z z}^{\mathrm{p} 2}\right)^{1 / 2} \\
& \text { dans le cas de la traction }, \\
\text { et }: \quad a_{0}= & \left(R_{0}^{\mathrm{p}}+R^{\mathrm{p}}\right)\left(1+2 A_{0}^{+} \varepsilon_{z \vartheta}^{\mathrm{p} 2}\right)^{1 / 2} \\
b_{0}= & R_{0}^{\mathrm{p}}+R^{\mathrm{p}} \\
& \text { dans le cas de la torsion }
\end{array}\right\}
$$

L'effet croisé est directement lié au seul paramètre nouveau $A_{0}^{+}$, et pour $A_{0}^{+}>0$, la surface s'étend plus rapidement dans la direction qui est perpendiculaire à celle du chargement, cet effet étant linéairement croissant avec la déformation cumulée $p$. On rappelle que $R^{\mathrm{p}}$ est également une fonction non linéaire croissante de $p$ (Eq. (104)).

Si ce modèle présente l'avantage d'être simple et de modéliser correctement les effets croisés, une comparaison qualitative des surfaces seuil issues de ce modèle avec les surfaces expérimentales révèle un très net désaccord.

Une démarche beaucoup plus sophistiquée due à Williams et Swensson [102, 103] est d'aborder le problème au niveau local, c'est-à-dire de considérer l'élément de volume comme une structure constituée d'une matrice élastoplastique et d'inclusions sphériques, puis d'évaluer la fonction de répartition des déformations locales autour de celles-ci. Sans entrer dans les détails des calculs fort longs, un formalisme local du type de celui développé Baltov et Sawczuk, combiné à une étude statistique de la fonction de répartition des déformations locales, conduit au modèle macroscopique global :

$$
\begin{aligned}
f & =\left\{\frac{3}{2}\left(I_{i j k l}+A_{0}^{+} \varepsilon_{i j}^{\mathrm{p}} \varepsilon_{k l}^{\mathrm{p}}\right) \times\right. \\
& \times\left(\sigma_{i j}^{\prime}+\varepsilon_{i j}^{\mathrm{p}}\left(L_{p q r s} \sigma_{p q} \sigma_{r s}-a\right)\right) \\
& \left.\times\left(\sigma_{k l}^{\prime}+\varepsilon_{k l}^{\mathrm{p}}\left(L_{p q r s} \sigma_{p q} \sigma_{r s}-a\right)\right)\right\}^{1 / 2}-R_{0}^{\mathrm{p}} .
\end{aligned}
$$

On notera que dans cette expression, la contrainte interne s'exprime sous une forme complexe, qui est la somme de deux composantes tensorielles, ce qui rejoint l'approche de Philipps $[110,111]$ évoquée cidessous.

Dans le cas de la traction, ou de la torsion, moyennant des conditions sur l'admissibilité physique de l'opérateur du $4^{\mathrm{e}}$ ordre $L_{i j k l}$ [102], on obtient une réelle distorsion, à savoir un effet de pointe dans la direction du chargement et un aplatissement dans la direction opposée. Cependant, les conditions obtenues sur cet opérateur dans le cas de la traction puis de la torsion, sont incompatibles. De plus, si

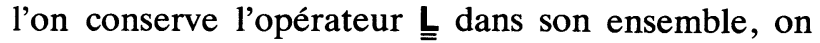
montre que la relation (162) n'apporte aucune distorsion significative de la surface $f$.

Une autre approche, due à Lee et Zawerl [108, 109], simple dans sa formulation mais très difficile à identifier, consiste à généraliser la loi d'évolution de l'écrouissage isotrope (Eq. (102)) à chacune des composantes du tenseur $\underline{M}$. On imagine facilement la difficulté expérimentale de l'identification.

Une autre approche phénoménologique prometteuse et historiquement basée sur les idées de Philipps $[110,111]$ consiste à définir directement une fonction représentative de l'état de distorsion dans l'équation de la surface seuil, afin de produire une perturbation autour de cette dernière [25, 110-112]. Si $R_{i j}^{\mathrm{p}}\left(\sigma_{i j}^{\prime}, X_{i j}^{\mathrm{p}^{\prime}}\right)$ est cette fonction de distorsion, on définit la surface $f$ sous la forme :

$$
\begin{array}{r}
f=\left\{\frac{3}{2}\left(\sigma_{i j}^{\prime}-X_{i j}^{\mathrm{p}^{\prime}}-R_{i j}^{\mathrm{p}}\right)\left(\sigma_{i j}^{\prime}-X_{i j}^{\mathrm{p}^{\prime}}-R_{i j}^{\mathrm{p}}\right)\right\}^{1 / 2}- \\
-R_{0}^{\mathrm{p}}
\end{array}
$$

La variable interne cinématique est donc la somme des deux composantes $X_{i j}^{\mathrm{p}}$ et $R_{i j}^{\mathrm{p}}$, ce qui est également le cas de la formulation (162) et du modèle LMAB (Eq. (46)). A ce niveau, on peut évoquer le modèle d'Eisenberg $[112,113]$ qui consiste à décomposer la surface en deux demi-surfaces et de leur affecter deux dimensions caractéristiques. Cette modélisation suppose l'absence d'effet croisé. $R_{i j}^{p}$ est défini par ces deux dimensions scalaires affectées d'un tenseur unité définissant les directions des distorsions. Chacune des dimensions croît ou décroît isotropement avec la déformation cumulée $p$ et la surface totale est la résultante de la déformation des deux demisurfaces, ce qui permet de décrire d'une manière indépendante les effets de pointe dans le sens du chargement et d'aplatissement dans le sens opposé. Le cas du modèle LMAB représente un cas simple de ce modèle dans lequel il n'existe aucune dimension privilégiée, toutes croissent d'une manière isotrope avec $p$.

Contrairement au modèle d'Eisenberg, dans la formulation de Rousset [25], $R_{i j}^{\mathrm{p}}$ est définie à partir de la variable cinématique $X_{i j}^{\mathrm{p}}$ et du cosinus de l'angle formé entre la direction actuelle d'écrouissage 
$\left(\frac{X_{i j}^{\mathrm{p}}}{J_{2}\left(\underline{\underline{\underline{X}}}^{\mathrm{p}}\right)}\right)$ et la direction d'écoulement en l'absence de distorsion $\left(\frac{\sigma_{i j}^{\prime}-X_{i j}^{\mathrm{p}^{\prime}}}{J_{2}\left(\underline{\mathbf{\sigma}}-\underline{\mathbf{X}}^{\mathrm{p}}\right)}\right)$. Dans cette optique, $R_{i j}^{\mathrm{p}}$ s'écrit :

$$
\begin{array}{r}
\dot{R}_{i j}^{\mathrm{p}}=\gamma^{+}\left|\left(\frac{\sigma_{i j}^{\prime}-X_{i j}^{\mathrm{p}^{\prime}}}{J_{2}\left(\underline{\mathbf{\sigma}}-\underline{\mathbf{X}}^{\mathrm{p}}\right)}\right)\left(\frac{X_{i j}^{\mathrm{p}^{\prime}}}{J_{2}\left(\underline{\mathbf{X}}^{\mathrm{p}}\right)}\right)\right|^{n^{+}} \times \\
\times \dot{X}_{i j}^{\mathrm{p}}-\Delta R_{i j}^{\mathrm{p}} J_{2}\left(\dot{X}^{\mathrm{p}}\right) .
\end{array}
$$

Les coefficients $\gamma^{+}$et $n^{+}$, tous deux positifs, permettent de mieux ajuster la forme des surfaces déformées. Dans le premier terme de droite de cette équation, $R_{i j}^{\mathrm{p}}$ est liée de façon linéaire à la variable cinématique donc tant que $X_{i j}^{\mathrm{p}}$ est activée, la fonction distorsion l'est également, ce qui est contraire à l'expérience où l'on observe une tendance à la saturation de la distorsion pour des chargements monotones croissants [25]. Pour tenir compte de cet effet, c'est-à-dire borner l'évolution de $R_{i j}^{\text {p }}$ sans affecter a priori la convexité des surfaces, Rousset introduit un terme de rappel proportionnel à $R_{i j}^{\mathrm{p}} J_{2}\left(\underline{\mathrm{X}}^{\mathrm{p}}\right)$, et ainsi $R_{i j}^{\mathrm{p}}$ tend asymptotiquement vers $1 / \Delta$ pour des chargements croissants (Eq. (164)).

La description complète des surfaces de charge repose sur une extension de la version plastique du modèle ONERA-LMT Cachan, à une seule variable cinématique, soit avec la définition thermodynamique des variables (Eqs. (14), (22), (23)) :

$$
\begin{aligned}
f & =\left\{\frac{3}{2}\left(\sigma_{i j}^{\prime}-X_{i j}^{\mathrm{p}^{\prime}}-R_{i j}^{\mathrm{p}^{\prime}}\right)\left(\sigma_{i j}^{\prime}-X_{i j}^{\mathrm{p}^{\prime}}-R_{i j}^{\mathrm{p}^{\prime}}\right)\right\}^{1 / 2}-R^{\mathrm{p}}-R_{0}^{\mathrm{p}} \\
\dot{\varepsilon}_{i j}^{\mathrm{p}} & =\dot{\lambda} \frac{\partial f}{\partial \sigma_{i j}}=\dot{\lambda}\left(\frac{\sigma_{k l}^{\prime}-X_{k l}^{\mathrm{p}^{\prime}}-R_{k l}^{\mathrm{p}}}{J_{2}\left(\underline{\mathbf{\sigma}}-\underline{\mathbf{X}}^{\mathrm{p}}-\underline{\mathbf{R}}^{\mathrm{p}}\right)}\right)\left(\delta_{k i} \delta_{l j}-\frac{\partial R_{k l}^{\mathrm{p}}}{\partial \sigma_{i j}}\right) \\
\dot{X}_{i j}^{\mathrm{p}} & =\frac{2}{3} a \dot{\varepsilon}_{i j}^{\mathrm{p}}-C \dot{\lambda} \frac{\sigma_{k l}^{\prime}-X_{k l}^{\prime}-R_{k l}^{\mathrm{p}}}{J_{2}\left(\underline{\boldsymbol{\sigma}}-\underline{\mathbf{X}}^{\mathrm{p}}-\underline{\mathbf{R}}^{\mathrm{p}}\right)} \cdot\left(\frac{\partial R_{k l}^{\mathrm{p}}}{\partial \sigma_{i j}}+\frac{\partial R_{k l}^{\mathrm{p}}}{\partial X_{i j}^{\mathrm{p}}}\right) \\
\dot{R}^{\mathrm{p}} & =b\left(Q_{1}-R^{\mathrm{p}}\right) \dot{p} \text { et } R_{i j}^{\mathrm{p}} \text { donné } \operatorname{par}(164)
\end{aligned}
$$

$\dot{\lambda}$ est déterminé par la relation de consistance $f=\dot{f}=0$.

On peut noter que la première hypothèse de normalité de la déformation plastique à la surface de charge, même distordue, est expérimentalement très bien vérifiée. Avec une telle approche, Rousset montre que l'on peut quantitativement assez bien décrire les effets croisés, les effets de pointe et d'aplatissement des surfaces pour des chargements radiaux et enfin les distorsions souvent complexes consécutives à des écrouissages de traction et de torsion combinés [25]. Pour conclure définitivement sur les possibilités d'une telle approche, il reste à savoir si elle est effectivement apte à rendre compte des courbes d'écrouissage cyclique sous chargement fortement non proportionnel (paragraphe ci-dessous) et dans l'affirmative de l'étendre au cas des matériaux fortement visqueux, tel que le 17-12 SPH. En ce qui concerne l'approche multicouche, on peut signaler l'application bidimensionnelle du modèle LMX (chaque couche possède un seuil d'écoulement bidimensionnel), avec une matrice élastique B complète (Eq. (57b)), qui conduit à une surface de charge définie par l'intersection des deux domaines élastiques locaux, c'est-à-dire de deux ellipses dans le plan des contraintes principales [37]. Cette description n'est pas sans rappeler le modèle d'Eisenberg où la surface de charge est également la résultante de :'intersection de deux ellipses.

En conclusion, dans ce paragraphe, on vient de montrer les multiples possibilités des modèles à variables internes quant à l'appréhension des distorsions des surfaces de charge, la solution retenue ne pouvant être assujettie qu'à une étude expérimentale détaillée permettant de cerner les propriétés fondamentales des distorsions.

7.2.2 Cas des chargements cycliques hors phases. Particulièrement dans le cas du 17-12 SPH sollicité en traction-torsion cyclique entre déformations imposées, le déphasage entre les composantes de la déformation altère très fortement la courbe de consolidation cyclique [105, 107, 114] (Fig. 34). En ,effet, sur la figure 34 , on s'aperçoit que si pour des sollicitations unidimensionnelles de traction ou de torsion, et bidimensionnelles radiales, la courbe de consolidation au sens de Von-Misès $\left(\frac{\Delta \sigma_{M}}{2}=\right.$ $\left.f\left(\bar{\varepsilon}_{\mathrm{M}}^{\mathrm{P}}\right)\right)$ est unique, il apparaît systématiquement un accroissement de durcissement sitôt que les composantes de la déformation sont déphasées. Sur la figure 34, dans le cas de déformations sinusoïdales $\left(\varepsilon_{z z}=\varepsilon_{z z \mathrm{~m}} \sin \omega t\right.$ et $\left.\gamma_{z \vartheta}=\gamma_{z \vartheta \mathrm{m}}(\sin \omega t-\varphi)\right)$, les deux paramètres caractérisant les sollicitations sont l'angle de déphasage $\varphi$ et le rapport des amplitudes, $\rho=\frac{\gamma_{z \vartheta \mathrm{m}}}{\sqrt{3} \varepsilon_{z z \mathrm{~m}}}$. Les propriétés de ce durcissement supplémentaire, chiffré par la différence entre la contrainte équivalente maximale et la contrainte équivalente correspondant au chargement cyclique 


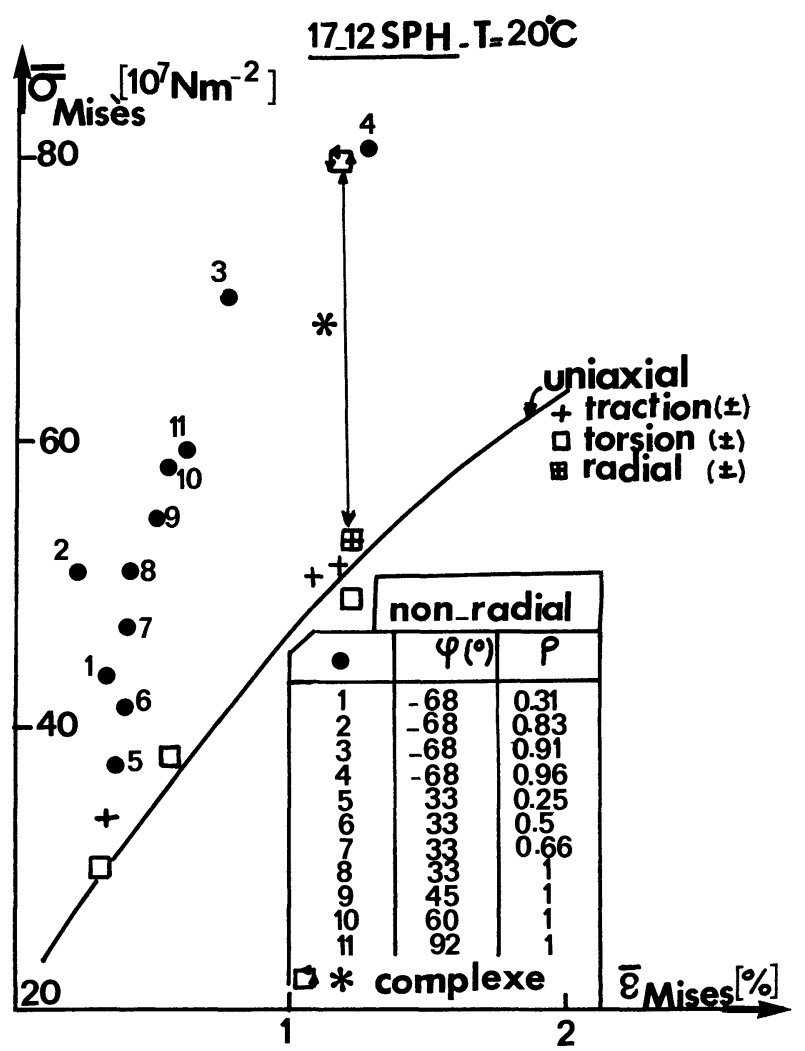

Fig. 34. - Acier 17-12 SPH à l'ambiante ; mise en évidence du durcissement cyclique supplémentaire dû au déphasage entre les déformations imposées de traction et de torsion. Influence de l'angle de déphasage $\varphi$. (D'après Benallal, Cailletaud, Chaboche, Marquis, Nouailhas et Rousset [107]).

[17-12 SPH at room temperature ; evidence of a supplementary cyclic hardening due to the phase difference between the imposed tensile and torsional strains. Part taken by the phase difference $\varphi$. (From Benallal, Cailletaud, Chaboche, Marquis, Nouailhas and Rousset [107]).]

$1 \mathrm{D}$, pour une déformation équivalente fixée, sont telles que :

- pour un déphasage fixé, le durcissement supplémentaire est d'autant plus conséquent que le rapport $\rho$ est grand,

- pour un rapport $\rho$ fixé $(\rho=1)$, le durcissement supplémentaire croît avec l'angle $\varphi$. Cependant, le maximum de variation est obtenu pour $30 \leqq \varphi \leqq$ $60^{\circ}$, au-delà de ces limites, le déphasage n'a que peu d'influence,

- pour des chargements plus complexes (carré, étoiles, ...) $[105,114]$, les points obtenus (Fig. 34) se situent toujours entre la courbe cyclique $1 \mathrm{D}$ et la courbe correspond au maximum de déphasage $\left(\varphi=90^{\circ}\right)$,

- si ce durcissement est rapidement créé, de l'ordre d'une vingtaine de cycles, il peut être totalement mais très lentement évanescent. Cette pro- priété est mise en évidence en réalisant en premier lieu une consolidation sous sollicitations hors phase $\left(\varphi=90^{\circ}\right)$, puis ensuite une sollicitation unidimensionnelle $(\varphi=0)$ et où l'on observe que la contrainte est très lentement décroissante avec le nombre de cycles. Cette propriété n'est pas sans rappeler l'évanescence très lente de la mémoire de la prédéformation maximale pour un cyclage sous des niveaux décroissants.

Physiquement, comme pour la distorsion des surfaces de charge, ce durcissement supplémentaire est dû à l'activation de nouveaux systèmes de glissement pouvant interagir entr'eux. Ce phénomène a reçu une attention particulière dans le cas des monocristaux sollicités suivant deux orientations successives et où suivant la seconde orientation, un écrouissage supplémentaire, dû à l'activation d'un autre système de glissement, est clairement observé [115].

La modélisation de tels effets n'est pas simple $a$ priori puisqu'elle est intimement liée à la description des surfaces de charge, qui, comme nous l'évoquions précédemment, n'est pas aisée. Cependant, l'étude des surfaces de charge au cours de la consolidation cyclique sous sollicitations hors phase [25], révèle clairement que pour un même point du cycle imposé (en déformation), les surfaces distordues croissent sensiblement homothétiquement. Ainsi, en tenant compte de cette observation et en faisant abstraction de la distorsion, une façon simple d'introduire la notion de sollicitation hors phase est d'introduire dans le paramètre asymptotique $Q_{1}^{\text {sat }}$ de la variable isotrope, une fonction simple caractéristique du déphasage. A ce niveau, il faut remarquer que pour des chargements non radiaux, l'ensemble des tenseurs des variables internes ne sont pas colinéaires et qu'une façon simple de caractériser le déphasage est de choisir un angle lié au degré de non-colinéarité de deux variables internes tensorielles, choisies plus ou moins arbitrairement.

Ainsi, dans le modèle ONERA, on voit apparaître naturellement l'angle $\alpha_{1}$ défini par les normales aux surfaces $f$ et $G$ (Eqs. (107), (108)) et tel que :

$$
\begin{aligned}
\cos \alpha_{1} & =\left\langle{\underline{\underline{n} 11^{*}}}^{*}\right. \\
& =\left\langle\left(\frac{\sigma_{i j}^{\prime}-X_{i j}^{\mathrm{v}^{\prime}}}{J_{2}\left(\underline{\boldsymbol{\sigma}}-\underline{\mathbf{X}}^{\mathrm{v}}\right)}\right)\left(\frac{\varepsilon_{i j}^{\mathrm{v}}-\xi_{i j}}{I_{2}\left(\underline{\boldsymbol{\varepsilon}}^{\mathrm{v}}-\underline{\boldsymbol{\xi}}\right)}\right)\right\rangle .
\end{aligned}
$$

L'équation (109) fixant l'évolution de $\dot{q}$ donc de $Q_{1}$ et dans laquelle intervient cet angle, prévoit effectivement un durcissement cyclique supplémentaire puisque le rapport $\left(\frac{q \text { hors phase }}{q \text { en phase }}\right)$ tend au maximum vers 1,4 [116]. Une modification astucieuse de la définition de $\dot{q}$ (Eq. (109)) telle que celle adoptée par Rousset (Eq. (166)) (introduction d'un facteur multiplicatif ou d'une élévation à la puissance) devrait permettre d'approcher la valeur expé- 
rimentale de ce rapport qui est compris en 4 et 5 (Fig. 34). Dans le modèle LMAB, il apparaît le même angle $\alpha_{1}$ et puisque la définition de $Q_{1}$ est identique à celle du modèle ONERA mais que $\underline{\mathbf{X}}^{\mathrm{vM(2)}}$ est assujettie aux variations de $R^{\mathrm{v}}$ donc de $Q_{1}$ (Eq. (117a)), il devrait apparaître un durcissement supplémentaire sensiblement deux fois plus élevé que celui engendré par le modèle ONERA (on doit noter qu'aucun calcul n'a été réalisé en ce sens). Comme précédemment, une modification dans la définition de la variable $\dot{q}$ devrait conduire à plus de lattitude dans l'ajustement du coefficient maximum de durcissement supplémentaire.

On rappelle que dans la fonction de distorsion $R_{i j}^{\mathrm{P}}$ introduite par Rousset, il intervient l'angle $\alpha_{2}$ tel que :

$$
\cos \alpha_{2}=\left(\frac{\sigma_{i j}^{\prime}-X_{i j}^{\mathrm{p}^{\prime}}}{J_{2}\left(\underline{\mathbf{\sigma}}-\underline{\mathbf{X}}^{\mathrm{p}}\right)}\right)\left(\frac{X_{i j}^{\mathrm{p}^{\prime}}}{J_{2}\left(\underline{\mathbf{X}}^{\mathrm{p}}\right)}\right) .
$$

La voie adoptée par Benalall et al. [107] est dans son fondement analogue aux précédentes et consiste à définir une fonction simple $A^{*}$ d'un angle $\alpha_{3}$, affectant la valeur à saturation de la variable $Q_{1}$. Ces auteurs choisissent l'angle $\alpha_{3}$ formé entre les tenseurs des vitesses des déformations et des contraintes déviées, soit :

$$
\cos \alpha_{3}=\left(\frac{\dot{\varepsilon}_{i j}^{v}}{I_{2}\left(\dot{\underline{\dot{\varepsilon}}}^{v}\right)}\right)\left(\frac{\sigma_{i j}^{\prime}}{J_{2}(\underline{\mathbf{g}})}\right) .
$$

Cet angle variant entre $-\pi / 2$ et $+\pi / 2$, la fonction $A^{*}$ est écrite sous la forme :

$$
\left.\begin{array}{l}
A^{*}=1-\cos ^{2} \alpha_{3}=\sin ^{2} \alpha_{3} \\
\text { donc } \quad 0 \leqq A^{*} \leqq 1
\end{array}\right\}
$$

La variable $Q_{1}$ est telle que :

$$
\left.\begin{array}{rlrl}
\dot{Q}_{1} & =D\left(A^{*}\right)\left(Q_{1}^{\text {sat }}\left(A^{*}\right)-Q_{1}\right) \dot{v} \\
\text { avec } & D\left(A^{*}\right) & =\left(d^{*}-f^{*}\right) A^{*}+f^{*} \\
\text { et } & Q_{1}^{\text {sat }}\left(A^{*}\right) & =\frac{g^{*} A^{*} Q_{\infty}^{\text {sat }}+\left(1-A^{*}\right) Q_{0}^{\text {sat }}}{g^{*} A^{*}+\left(1-A^{*}\right)}
\end{array}\right\},
$$

$d^{*}, f^{*}, g^{*}, Q_{\infty}^{\text {sat }}$ et $Q^{\text {sat }}$ sont des constantes dépendant du matériau. On peut s'apercevoir que si $A^{*}=0 \quad$ (essai proportionnel), $\dot{Q}_{1}=f^{*}\left(Q_{0}^{\text {sat }}-\right.$ $\left.Q_{1}\right) \dot{v}$, et la valeur à saturation est fixée à $Q_{0}^{\text {sat }}$. Par contre, si $A^{*}=1$ (déphasage maximum), $\dot{Q}_{1}=$ $d^{*}\left(Q_{\infty}^{\text {sat }}-Q_{1}\right) \dot{v}$, et la valeur asymptotique est égale à $Q_{\infty}^{\text {sat }}$. Le durcissement supplémentaire maximum est donc totalement maîtrisable et égal à $\left(Q_{\infty}^{\text {sat }}-\right.$ $\left.Q_{0}^{\text {sat }}\right)$, les valeurs des coefficients $f^{*}$ et $d^{*}$ fixant les vitesses d'évolution de $\dot{Q}_{1}$. Le système (170) prévoit également l'évanescence de ce durcissement supplé- mentaire lorsque l'on retourne à des conditions radiales $\left(Q_{1}^{\text {sat }}\right.$ peut décroître de $Q_{\infty}^{\text {sat }}$ à $\left.Q_{0}^{\text {sat }}\right)$.

L'introduction des équations (168) à (170) dans le modèle ONERA-LMT Cachan sous une forme simplifiée :

$$
\begin{aligned}
\dot{\varepsilon}_{i j}^{\mathrm{v}} & =\frac{3}{2}\left\langle\frac{J_{2}\left(\underline{\mathbf{g}}-\underline{\mathbf{X}}^{\mathrm{v}}\right)-R^{\mathrm{v}}-R_{0}^{\mathrm{v}}}{K}\right\rangle^{n^{*}} \frac{\sigma_{i j}^{\prime}-X_{i j}^{\mathrm{v}}}{J_{2}\left(\underline{\mathbf{g}}-\underline{\mathbf{X}}^{\mathrm{v}}\right)} \\
\dot{X}_{i j}^{\mathrm{v}} & =\frac{2}{3} a \dot{\varepsilon}_{i j}^{\mathrm{v}}-C(v) X_{i j}^{\mathrm{v}} \dot{v} \quad \text { avec } \\
C(v) & =C_{\mathrm{s}}+\left(1-C_{\mathrm{s}}\right) \exp -b_{\mathrm{s}} v \\
\dot{R}^{\mathrm{v}} & =b\left(Q_{1}-R^{\mathrm{v}}\right) \dot{v}
\end{aligned}
$$

permet de rendre compte quantitativement, d'une manière assez simple mais réaliste, en faisant abstraction de la distorsion des surfaces, de l'ensemble des effets liés au déphasage évoqués en début de paragraphe. Cependant, comme pour les essais 1D, pour un déphasage donné, si l'on augmente le niveau de déformation après saturation sous un premier niveau, le nouvel état stabilisé n'est atteint qu'après une nouvelle période de consolidation, ce qui n'est pas prévu par cette approche où le second état stabilisé est directement atteint au premier cycle. En conséquence, il nous semble plus judicieux de faire intervenir la fonction de déphasage sur la variable $\dot{q}$ affectant $\dot{Q}_{1}$ (définition de la surface $G$ ) en adoptant éventuellement un formalisme voisin du système (170) et en choisissant astucieusement l'angle $\alpha$ (Eqs. (166), (167), (168), ... ?).

Il nous semble très important de signaler que l'INCO 718 qui déconsolide cycliquement présente également un durcissement supplémentaire lié au déphasage des sollicitations et l'effet croisé est toujours visible lorsque l'on change la direction du cyclage à déformation équivalente constante [117]. L'analyse des résultats expérimentaux n'est pas aisée car ce matériau s'adoucit aussi bien pour les essais en phase, qu'hors phase, mais globalement une formulation telle que celle qui vient d'être exposée (Eqs. (168) à (171)) nous semble tout à fait transposable, sans modification majeure.

En ce qui concerne les modèles multicouches, l'absence de documents ou de calculs réalisés en ce sens, ne nous permet pas de conclure quant à leur possibilité vis-à-vis des essais cycliques hors phase, mais a priori, compte tenu des lacunes que présentent ces formulations, envers les effets cycliques $1 \mathrm{D}$, des améliorations assez conséquentes devront être apportées.

7.2.3 Etude du rochet $2 D$ de traction-torsion. Depuis quelques années, un très vif intérêt, particulièrement par l'école française, a été porté à l'étude du phénomène de rochet $2 \mathrm{D}$ de traction-torsion [13, $33-34,47,74,118-120]$, et ceci pour deux raisons essentielles : 
- sa relative simplicité expérimentale qui consiste à superposer à une déformation primaire cyclique de torsion, à vitesse imposée $\left( \pm \dot{\varepsilon}_{z \vartheta}^{\mathrm{T}}\right)$, entre déformations fixées $\left( \pm \varepsilon_{z \vartheta}^{T}\right)$, un très faible chargement statique secondaire de traction $\sigma_{z z}$. On enregistre les évolutions simultanées de la contrainte de torsion $\sigma_{z \vartheta}$ et de la déformation axiale cumulée $\varepsilon_{z z}$, en fonction du nombre de cycles $N_{\mathrm{c}}$,

- la sévérité de ce type de sollicitation (composantes statique et cyclique) vis-à-vis des modèles identifiés, le plus fréquemment, sous sollicitation uniaxée [118].

Dans le cas du 17-12 SPH où l'on commence à posséder une base expérimentale conséquente [13, 33, 74], les principales caractéristiques obtenues sont :

- Pour un isotherme et une amplitude de déformation fixés, la déformation axiale présente une phase de consolidation primaire, sensiblement associée à l'établissement de la contrainte stabilisée $\sigma_{z \vartheta}^{\text {stab. }}(N \simeq 100$ cycles $)$, suivie d'une période quasiment stationnaire $\left(N_{\mathrm{c}}=700\right.$ cycles $)$ et dont la vitesse croît avec la contrainte axiale $\sigma_{z z}$. On peut noter qu'indépendamment de la température, l'accommodation n'a jamais été obtenue. On montre de plus, qu'il n'existe aucun effet de mémoire lorsqu'on réalise des essais à niveaux décroissants de $\sigma_{z z}$, pourvu que le nombre de cycles soit suffisamment important et ceci, indépendamment de la température considérée.

- Si l'on supprime la contrainte axiale, il apparaît systématiquement une phase de rochet négatif $\left(\dot{\varepsilon}_{z z} \leqq 0\right)$ associé à un changement dans le sens de la courbure des réponses $\varepsilon_{z z}=f\left(\varepsilon_{z \vartheta}\right)$. Cette observation est tout à fait conforme à l'hypothèse de normalité à des équipotentielles translatées cinématiquement.

- Pour un isotherme et une contrainte axiale fixés, la vitesse de déformation axiale est d'autant plus grande que l'amplitude de déformation imposée $\varepsilon_{z \vartheta}$ est élevée. Si l'on réalise des essais à niveaux de déformation croissants, il apparaît à chaque niveau, systématiquement une nouvelle consolidation cyclique associée à une nouvelle phase transitoire d'écoulement axial, débouchant sur une zone d'écoulement sensiblement stationnaire. A l'inverse, comme on l'a déjà mentionné précédemment et à l'exemple de la contrainte axiale, il n'existe aucun effet de mémoire permanente de la prédéformation (du moins pour des prédéformations inférieures à $1,5 \%$ ) pourvu que l'on cycle suffisamment longtemps $\left(N_{\mathrm{c}} \geqq 600\right)$ sous le plus faible niveau de déformation, la contrainte de torsion et la vitesse axiale retournent progressivement vers les valeurs qu'elles auraient atteintes si l'on avait directement sollicité le matériau sous le plus faible niveau. Ces observations sont en accord avec les propriétés cycliques $1 \mathrm{D}$ et justifient à nouveau l'introduction de la surface de non-écrouissage $G$ et des variables internes qui lui sont associées.

- Le point le plus important est la très grande sensibilité de la vitesse axiale $\dot{\varepsilon}_{z z}$ à la vitesse de cisaillement $\dot{\varepsilon}_{z \vartheta}^{\mathrm{T}}$, et l'on montre qu'un rapport de $10^{3}$ sur cette dernière entraîne un facteur variant de 50 à 100 , suivant la température $\left(20 \leqq T \leqq 650^{\circ} \mathrm{C}\right)$, sur la vitesse axiale pour un nombre de cycles donné $[74,120]$. Ce fait montre que la composante visqueuse a un rôle déterminant sur le rochet axial et que par conséquence, seuls des modèles viscoplastiques peuvent prétendre décrire les phénomènes observés. Cette conclusion est étayée par le constat que la vitesse axiale déterminée pour $N_{\mathrm{c}} \simeq$ 1000 cycles, en fonction de la température, présente une évolution parallèle à celle de la composante visqueuse (Fig. 32) et en particulier un minimum entre 300 et $400{ }^{\circ} \mathrm{C}$ [74].

- On peut également mentionner qu'en accord avec l'analyse présentée au paragraphe concernant les effets liés aux interactions dislocations-défauts ponctuels, l'exposant de sensibilité de la contrainte

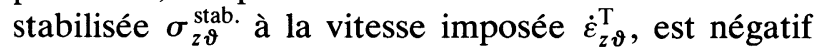
pour la zone $300-500{ }^{\circ} \mathrm{C}$. De plus, on montre que dans cet intervalle de température, la consolidation cyclique est maximum lorsque la composante visqueuse est minimum.

$\mathrm{Si}$ qualitativement toutes les observations qui viennent d'être énumérées peuvent être prises en compte à l'aide des différents formalismes développés, à notre connaissance, quantitativement, aucun modèle n'a encore été identifié au regard de l'ensemble de ces résultats qui présentent une richesse et une diversité peu commune et réalisent une synthèse des difficultés que l'on peut rencontrer dans la modélisation, tous les effets étant présents et intimement liés; comportement viscoplastique, sollicitation 2D, effets de normalité, de mémoire évanescente de l'histoire antérieure, du temps, de la température et de la configuration des défauts ponctuels dans le champ de contrainte des dislocations. Actuellement, les auteurs des approches ONERA-LMT Cachan, LMAB, EMP et CEA travaillent sur leur propre modèle, afin d'appréhender correctement le rochet de traction-torsion. En effet, jusqu'à présent, il s'avère que la plupart des modèles, souvent pris dans des versions très dépouillées [33, 119], surestiment la déformation axiale [118-119]. A notre avis, compte tenu de la complexité de ce phénomène, pour conclure définitivement ou apporter d'éventuelles modifications (par exemple sur les termes d'écrouissage des variables) aucun terme ne doit être omis, surtout pas les termes de restauration et l'hypothèse de la surface de non-écrouissage, lorsqu'on veut modéliser des expériences à $600^{\circ} \mathrm{C}$ sur le 17-12 SPH avec des modèles globaux! 
En ce qui concerne le modèle multicouche du CEA qui conduit à une bonne description du rochet $2 \mathrm{D}$ à $650^{\circ} \mathrm{C}$ [33-34], il ne peut que présenter des défaillances aux plus basses températures puisqu'il n'apparaît aucun terme visqueux (ce qui peut même paraître paradoxal pour l'isotherme $650{ }^{\circ} \mathrm{C}$ ), la déformation axiale étant principalement décrite par les termes de restauration des variables cinématiques. Comme nous l'avons déjà évoqué, une écriture purement viscoplastique serait tout à fait souhaitable et renforcerait considérablement les possibilités et la crédibilité de ce type d'approche.

Pour conclure, on peut dire que la base expérimentale étant acquise, la confrontation des différents modèles dans leur forme initiale, aux réalités expérimentales du rochet $2 \mathrm{D}$, est entrée dans une phase très active devant déboucher assez rapidement sur des conclusions, voire des modifications, significatives.

En dernier lieu, on peut souligner que si l'aspect qualitatif des essais cycliques non symétriques, en contraintes conduisant au rochet 1D ou en déformations sur un ou plusieurs niveaux donnant le phénomène de relaxation de la contrainte moyenne [13-14, 121], est assez bien restitué par l'ensemble de ces modèles, il subsiste qualitativement de nombreux problèmes d'identification et en particulier celui de l'acuité du couplage des variables isotrope et cinématique. Ces problèmes sont actuellement en cours d'étude dans différents laboratoires universitaires et industriels.

\section{Conclusions générales.}

En conclusion, on peut affirmer que la souplesse du formalisme thermodynamique général des modèles à variables internes laisse de nombreuses latitudes quant au choix de la nature et du nombre des variables à prendre en compte, suivant la complexité des problèmes physiques des différents mécanismes de déformation en présence. Ainsi, tous les modèles présentés, du moins dans les versions les plus élaborées, reposent sur la définition, d'une part d'une équation viscoplastique d'état et d'autre part, d'au moins deux variables internes, une tensorielle cinématique $\underline{\mathbf{X}}^{\mathbf{v}}$ et une scalaire $R^{\mathrm{v}}$, l'ensemble du système d'équations obtenu étant traité d'une manière continue (modèles globaux) ou discrète (modèles multicouches). La (les) variable cinématique sert essentiellement à décrire les boucles d'hystérésis effort-déformation et la (les) variable isotrope l'évolution continue (croissante ou décroissante) du seuil d'écoulement avec la déformation viscoplastique et le temps. Nous avons montré que l'ensemble des approches, moyennant des modifications plus ou moins conséquentes sur certaines d'entr'elles, étaient capables, du moins qualitativement de modéliser la plupart des phénomènes observés sous sollicitation unidimensionnelle.

Pour tenir compte des effets microstructuraux, il est toujours possible d'introduire de nouvelles variables internes, étroitement liées à la physique du mécanisme que l'on veut représenter, et affectant la composante visqueuse $\sigma_{\mathrm{v}}$ et très certainement les deux variables de base $\underline{\mathbf{X}}^{\mathrm{v}}$ et $R^{\mathrm{v}}$.

Il est également possible, toujours en introduisant de nouvelles variables internes, d'appréhender assez simplement les distorsions des surfaces de charge ainsi que le durcissement cyclique supplémentaire dû au déphasage des sollicitations, ce secteur étant en pleine expansion. On terminera en constatant que la plupart de ces formulations sont encore mouvantes et en pleine phase de développement, et que l'étude du formalisme des lois viscoplastiques constitue, en France et particulièrement au sein du «GRECO-GIS, Rupture à Chaud » un secteur de recherche très actif.

\section{Remerciements.}

L'auteur remercie les différentes équipes matériaux des organismes, ONERA, EDF, SNECMA, CEA ainsi que le "GIS Rupture à Chaud », pour la mise à disposition des différentes informations en leur possession. Il en est de même pour les laboratoires du GRECO «Grandes Déformations et Endommagement ", à savoir le LMT Cachan, le LMSX et le centre des matériaux de l'EMP.

\section{Bibliographie}

[1] Lemaître, J., Сhaboche, J. L., Mécanique des Matériaux Solides (Dunod) 1985.

[2] Chaвoche, J. L., Continuum Damage Mechanics : Present State and Future Trends in «International Seminar on local approach of fracture », 3-5 juin (1986), Moret/s/Loing, (1986) pp. 53-78.

[3] Lemaître, J., Nucl. Eng. Des. 80 (1984) 233.

[4] Hayhurst, D. R., Dimmer, P. R. et Morrison, C. J., Philos. Trans. R. Soc. London A 311 (1984) 103.
[5] Boisserie, J. M., Guelin, P., Terriez, J. M. et WACK, B., J. Eng. Mater. Technol. 105 (1983) 155.

[6] Berveiller, M. et Zaoui, A., J. Eng. Mater. Technol. 106 (1984) 295.

[7] Delobelle, P., Thesis, Besançon, France, 1-302 (1985).

[8] Chaboche, J. L. et Rousselier, G., ASME, J. Press. Vess. Techn. 105 (1983) 153. 
[9] Delobelle, P. et Oytana, C., J. Nucl. Mater. 139 (1986) 204.

[10] Delobelle, P., Scripta Metall. 20 (1986) 61.

[11] Crouzet, D., P.V.D. 592 Mat/T43 EDF (1984).

[12] Pugh, C. E., Progress in Developping Constitutive Equations for Inelastic Design Analysis in «3rd Int. Sem. on Inelastic Analysis and Life Prediction in High Temperature Environment », Paris 24-25/08 (1981).

[13] Contesti, E., Rapport - Etude 2B - « Lois de comportement des aciers inoxydables en chargements combinés plasticité - fluage en uniaxial et biaxal », juin (1985).

[14] Marquis, D., Rapport - Etude 2B - Idem [13].

[15] De Langre, E. et Rousset, M., D.E.A., Ecole Polytechnique (1981).

[16] Chaboche, J. L., Dang-Van, K. et Cordier, G., Modelisation of the strain memory effect on the cyclic hardening of 316 stainless-steel, SMIRT 5, Division L, 79, Berlin (1979).

[17] Nouallhas, D., Modélisation macroscopique du comportement viscoplastique et des effets de vieillissements des aciers inoxydables. Rapport 57/1765 RY 095 R (1985).

[18] Halphen, B. et Nguyen, Q. S., J. Mec. Th. 14 (1975) 39.

[19] SidorofF, F., "Grandes Déformations », SophiaAntipolis, Rapport GRECO $n^{\circ} 51 / 82$ (1982).

[20] Снавосне, J. L., Thesis, Fac. Sci. Paris, France (1978).

[21] Germain, R., Nguyen, Q. S. et Suquet, P., $J$. Appl. Mech. 50 (1983) 1020.

[22] Ellis, J. R., Robinson, D. R. et Pugh, C. E., J. Eng. Mater. Technol. 105 (1983) 236.

[23] Moreton, D. N., Moffat, D. G. et Parkinson, D. B., J. Strain Analysis 16 (1986) 127.

[24] Baltov, A. et SawczucK, A., Acta Mech. 1 (1965) 81.

[25] Rousset, M., Thesis, ENSET, France, 1-153 (1985).

[26] Philipps, A., in «Plasticity in Structural Engineering Fundamentals and Applications ", CISM n²41. Ch. Massonet, W. Olszak (1979) 191.

[27] Oytana, C., Delobelle, P. et Mermet, A., J. Eng. Mater. Technol. 104 (1982) 1.

[28] Ponter, A. R. et Leckie, F. A., J. Eng. Mater. Technol. 98 (1976) 47.

[29] Murakami, S., J. Eng. Mater. Technol. 105 (1983) 99.

[30] Chrysochoos, A., J. Mec. Th. Appl. 4 (1985) 589 et Thèse Univ. Sci. Techn. du Languedoc (1983).

[31] Contesti, E., E.M.P. Compte rendu du GIS « rupture à chaud », Etude 2B (1984).

[32] ZhONG ZhANG, E.M.P. Compte rendu, « Travaux d'Option » (1985).

[33] Blanchard, P. et Tortel, J., Preprints of the 5th International Seminar on: «Inelastic Analysis and Life Prediction in High Temperature Environment », August 26-27 Paris (1985).

[34] BlanChaRd, P., Compte rendu du GIS « Rupture à Chaud» Etude 1.2 (1982).
[35] Zarka, J., Nguyen, Q. S. et Halphen, B., Plasticité et viscoplasticité, récents développements, IPSI Paris, mai (1978).

[36] MroZ, Z. et ZARKA, J., " Cyclic constitutive relations for metals " in Matériaux et structures sous chargement cyclique. Palaiseau 28-29 septembre 11-48 (1978).

[37] Engel, J. J., « Analyse d'un groupement à deux patins bidimensionnels soumis à un chargement cyclique », Idem à 36, 81-86 (1978).

[38] Armstrong, P. J. et Frederick, C. O., C.E.G.B. report RD/B/N 731 (1966).

[39] Delobelle, P. et Lexcellent, C., à publier.

[40] Delobelle, P., Oytana, C. et Mermet, A., Mechanical Behaviour of Materials IV Ed Carlsson, Ohlson (Pergamon Press) 1984, p. 141.

[41] Kujawski, D. et Mroz, Z., Acta Mech. 36 (1980) 213.

[42] Mroz, Z., J. Mech. Phys. Sol. 15 (1967) 163.

[43] Mroz, Z. et Trampczynski, W. A., Int. J. Solids Struct. 20 (1984) 467.

[44] Besseling, J. F., J. Appl. Mech. 25 (1953) 529.

[45] Meijers, P., Janssen, G. T. M. et BoolJ, J., SMIRT III, L 3/9/London (1975).

[46] Schmidt, C. G. et Miller, A. K., Res. Mech. 3 (1981) 109.

[47] Delobelle, P., Rapport Intermédiaire EDF (1986), à publier.

[48] Krempl, E., J. Eng. Mater. Technol. 101 (1979) 380.

[49] Cernocky, E. P. et Krempl, E., Acta Mech. 36 (1980) 263.

[50] PhilipPs, A., « Plasticity in Structural Engineering Fundamentals and Applications », CISM n ${ }^{\circ} 241$, Ch. Massonet, W. Olszak, 191 (1979).

[51] Valanis, K. C. et Lee, C. F., J. Appl. Mech. 51 (1984) 367.

[52] Poirier, J. P., Acta Metall. 25 (1977) 913.

[53] Lloyd, G. J. et McEllroy, R. J., Acta Metall. 22 (1974) 339.

[54] HART, E. W., Acta Metall. 25 (1977) 913.

[55] Dobès, F., Acta Metall. 28 (1980) 377.

[56] Oytana, C., Delobelle, P. et Mermet, A., $J$. Mater. Sci. 14 (1979) 549.

[57] Ahlquist, C. N. et NiX, W. D., Scripta Metall. 3 (1969) 679.

[58] Solomon, A. A., Rev. Sci. Instr. 40 (1969) 1025.

[59] Bolton, J. C., Central Electricity Board RD/B/N 2300, Berkeley Nuclear Laboratories (1973).

[60] Delobelle, P. et Oytana, C., Nucl. Eng. Des. 83 (1984) 333.

[61] Miller, A. K., J. Eng. Mater. Technol. 96 (1976) 97.

[62] Ohashi, Y., Ohno, N. et Kawai, M., J. Eng. Mater. Technol. 104 (1982) 159.

[63] Lemaître, J., Thèse d'Etat, Fac. Sci. Orsay (1971).

[64] Delobelle, P., Rapport Interne au LMAB, Travaux non publiés.

[65] Hollomon, J. H., Trans. AIME 162 (1945) 268.

[66] Voce, E., J. Inst. Metals 74 (1948) 537.

[67] Ludwigson, D. C., Metall. Trans. 2 (1971) 2925.

[68] Ghosh, A. K., Metall. Trans. ?8 (1977) 1221. 
[69] Grossetie, J. C., Boerman, D. et Piatti, G., SMIRT 8, Bruxelles 19-23 August, Division L, L8/1, 371 (1985).

[70] Murakami, S. et Ohno, N., Solids Struct. 18 (1982) 597.

[71] Ohashi, Y., Kawai, M. et Shimizu, H., J. Eng. Mater. Technol. 105 (1983) 257.

[72] Blanchard, P., Note Technique, CEA-SRMA, NT 85 1431, octobre (1985).

[73] Wire, G. L., Duncan, D. R., Cannon, N. S., Johnson, G. D., Alexopoulos, P. S., MuKHERJEE, S. et LI, C. Y., J. Eng. Mater. Technol. 103 (1981) 305.

[74] Delobelle, P. et Lexcellent, C., Travaux non publiés (Contrat EDF) (1986).

[75] OHNo, N., J. Appl. Mech. 49 (1982) 721.

[76] Goodall, I. W., Hales, R. et Walters, D. J., 3rd IUTAM Symposium, Leicester, U.K., Ed. A.R.S. Ponter et D. R. Haghurst, 8-12 septembre (1980).

[77] Delobelle, P. et Oytana, C., à paraître, SMIRT 9, L 10/2, Lausanne, 17-22 August (1987).

[78] GitTUs, J. H., Acta Metall. 26 (1978) 305.

[79] Mitra, S. K. et McLean, D., Proc. R. Soc. A 295 (1966).

[80] Mrtra, S. K. et McLean, D., Metall. Sci. J. 1 (1967) 192.

[81] Gasca-Neri, R., BarretT, C. R. et Nix, W. D., Scripta Metall. 5 (1971) 733.

[82] Williams, K. R. et Whilshire, B., Met. Sci. J. 7 (1973) 176.

[83] Strudel, J. L. et Blanc, D., Contrat EDF/Armines, mars (1984).

[84] Nouailhas, D., Compte rendu, Etude 2B du GIS « Rupture à Chaud », 28 juin (1985).

[85] Benallal, A. et Ben Cheikh, A., Proc. 2rd Int. Conf. on Constitutive Laws, Tucson, Arizona, 510 janvier (1987).

[86] Cailletaud, G. et Chaboche, J. L., ICM 3, Ed. K. J. Miller et R. F. Smith, Cambridge, England, 20-24 août (1979), vol. 2, 23 (1979).

[87] Weiss, B. et Stickler, R., Metall. Trans. 3A (1972) 851.

[88] Deleury, M. J., Donatti, J. R., Strudel, J. L., Ann. Chim. Fr. 6 (1981) 59.

[89] Schoeck, G. et Seeger, A., Acta Metall. 7 (1959) 469.

[90] Evans, J. T. et Douthwaite, R. N., Acta Metall. 21 (1973) 49.

[91] Strudel, J. L., dans Dislocations et déformation plastiques, Yravals 3-14 septembre (Editions de Physique) pp. 199-222.

[92] Nowick, A. S. et BERRY, B. S., dans Anelastic Relaxation in Crystalline Solids (Academic Press, New York) 1972, p. 176-247.

[93] Goodman, A. M., 4th Int. Sem. on Inelastic. Anal. and Life Pred. in High Temp. Env., Chicago, 2930 août (1983).
[94] De Paula e Silva, E., Beranger, G. et LACOMBE, P., Scripta Metall. 5 (1971) 795.

[95] De Paula e Silva, E., Com-Nougue, J., BeranGER, G., C.R.A.S. 272c (1971) 180.

[96] Reed Hill, R. E., Park, S. C. et Beckerman, L. P., Acta Metall. 31 (1983) 1715.

[97] Delobelle, P., Varchon, D. et Oytana, C., Metall. Trans. 16A (1985) 361.

[98] Rose, K. S. et Glover, S. G., Acta Metall. 14 (1966) 1505.

[99] Lebienvenu, M. et Dubois, B., J. Phys. Colloq. France 44 (1983) C9-439.

[100] Miller, A. K. et Sherby, O., Acta Metall. 26 (1978) 289.

[101] Park, S. C., Beckerman, L. P. et Reed-Hill, R. E., Metall. Trans. 14A (1983) 463.

[102] Williams, J. F. et Swensson, N. L., J. Strain Anal. 5 (1970) 2.

[103] Williams, J. F., Meccanica 6 (1971) 104.

[104] Edelman, F. et Drucker, D. C., J. Franklin Inst. 251 (1951) 581.

[105] Cailletaud, G., Kaczmarek, H. et Policella, H., Mec. Mat. 3 (1984) 4.

[106] KremPL, E. et Lu, H., J. Eng. Mater. Technol. 106 (1984) 376.

[107] Benallal, A., Cailletaud, G., Chaboche, J. L., Marquis, D., Nouailhas, D. et Rousset, M., $2^{\mathrm{e}}$ Int. Conf. on Biaxial-Multiaxial Fatigue, Sheffield, décembre (1985).

[108] Lee, D. et Zaverl, J. R., Acta Metall. 26 (1978) 1771.

[109] LeE, D. et ZAVerL, J. R., Metall. Trans. 11A (1980) 983.

[110] Phillips, A. et Weng, G. J., J. Appl. Mech. (1975) 375

[111] Weng, G. J. et Phillips, A., Int. J. Eng. Sçi. 15 (1977) 45.

[112] Eisenberg, M. A. et Chian-Fonc Yen, J. Eng. Mater. Technol. 106 (1984) 355.

[113] Eisenberg, M. A. et Chian-Fong Yen, J. Appl. Mech. 48 (1981) 276.

[114] Ohashi, Y., KaWai, M. et KaIto, T., J. Eng. Mater. Technol. 107 (1985) 101.

[115] Franciosi, P., Berveiller, M. et Zaoui, A., Acta Metall. 28 (1980) 273.

[116] Nouailhas, D., Policella, H. et KaczmareK, H., Rech. Aérosp. 6 (1982) 385.

[117] NouAIlHas, D., Documents ONERA non publiés.

[118] Inoue, T., IMATAMI, S., SAHASHI, T., SMIRT VIII, vol. L, L 1/2, août 19-23 Bruxelles (1985).

[119] Clément, G., GuionNet, C., SMIRT VIII, Vol. L, L 1/9, août 19-23, Bruxelles (1985).

[120] Delobelle, P., Lexcellent, C., Oytana, C., ICMV, Beijing, Chine, juin (1987).

[121] Engel, J. J., Rapport GIS, Etude 2B, « Lois de comportement des aciers inoxydables en chargements combinés plasticité-fluage en uniaxial et biaxial » (1985). 